\title{
Porous Aluminosilicate Inorganic Polymers: \\ a New Class of Heterogeneous Solid Acid Catalysts
}

\author{
by
}

Mohammad Issa Mousa Alzeer

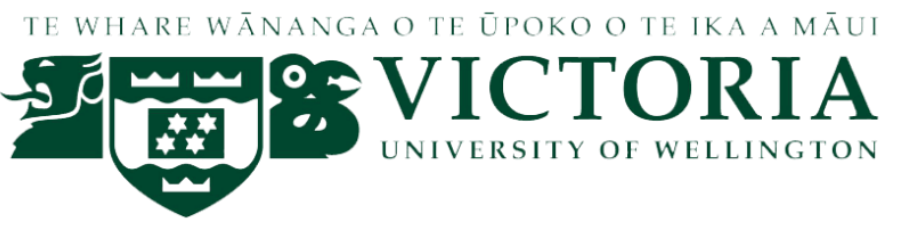

\begin{abstract}
A thesis
submitted to the Victoria University of Wellington

in fulfilment of the requirements for the degree of

Doctor of Philosophy

in Chemistry
\end{abstract}

Victoria University of Wellington

2017 


\begin{abstract}
Recent increased environmental awareness and the stimulus of greener chemistry has driven the rapid development of heterogeneous catalysts, particularly solid acids, for a wide range of organic synthesis applications. Typical homogenous acids suffer drastic drawbacks in terms of their corrosivity, toxicity, and reusability, in addition to their separation that generates large amounts of industrial wastes which exceeds in many cases the amount of the formed products.

Crystalline aluminosilicate inorganic polymers (zeolites) have successfully replaced the typical homogenous Lewis acids in many industrially important applications, the majority of which are in the petrochemical industries, e.g. production of olefins and aromatics. The fine chemical industries, however, are more challenging and still mainly use homogenous catalysts. Typical zeolite catalysts are hindered by their restricted micropores, and the low hydrothermal stability of other mesoporous M-silicates (such as MCM-41) results in structural deformation in aqueous solutions at elevated temperatures. Other highly promising solid catalysts suffer drawbacks of high cost, sophisticated synthesis procedures, and environmental risks from the use of toxic reagents. Thus, there is still a need for new cost-efficient reactive heterogeneous solid catalysts that are also environmentally benign.
\end{abstract}

This thesis reports the development of amorphous aluminosilicate inorganic polymers (known as geopolymers) as a novel class of heterogeneous solid acid catalysts. These geopolymers can be synthesised with the desired acidity and porosity in a very energyefficient and simple procedure which does not involve lengthy thermal treatments or the use of costly and sometimes toxic structural directing agents that are required for the synthesis of zeolite or other mesoporous aluminosilicates.

Microporous, mesoporous and hierarchical geopolymer-based catalysts were synthesised from different precursors with high surface area and acidic sites (Bronsted and Lewis) generated within their structure by ion-exchange with ammonium ions 
followed by thermal treatment, allowing the nature of these acidic sites to be tailored to specific applications. Furthermore, some of the resulting geopolymer catalysts were subjected to post synthetic treatments (demetallation) which provided improved acidity and porosity.

In the first instance, the geopolymer-based catalysts were synthesised from a naturally occurring clay mineral and their catalytic performance was evaluated in the industrially important Beckmann rearrangement of cyclohexanone oxime to $\varepsilon$-caprolactam. High catalytic reactivity and selectivity was achieved over the geopolymer-based catalysts that possess high surface area and weak surface acidities consisting of $\mathrm{H}$-bonded silanol nests and vicinal silanols.

The catalytic reactivity of the clay-based geopolymer catalysts was further evaluated in the Friedel-Crafts alkylation of large substituted arenes with benzyl halide as alkylating agent, where typical microporous zeolites show poor reactivity due to diffusional limitations. In this reaction, the thermal treatment was adjusted to generate the required Bronsted and Lewis acidic sites. High reactivity was achieved over several mesoporous geopolymer-based catalysts, with the best performance being observed over a hierarchical geopolymer-based catalyst that exhibits the highest acidity of all these new catalysts.

In another approach, highly reactive geopolymer-based catalysts were synthesised from industrial wastes precursors (fly ash). Several fly ashes were collected from different sources and the influence of their chemical and physical properties on the resulting geopolymers was investigated. These fly ash-based catalysts demonstrated excellent catalytic performance in the alkylation of benzene and substituted benzenes and their active sites were ascribed to a combination of $\mathrm{Fe}_{2} \mathrm{O}_{3}$ present in the raw fly ash, together with the Bronsted and Lewis acid sites that were generated within the geopolymers framework by the ion-exchange process followed by thermal treatment.

The use of the fly ash-based catalysts was also demonstrated in another highly demanding catalytic process, the Friedel-Crafts acylation of aromatics. High reactivity and selectivity was achieved in the acylation reactions of anisole and mesitylene using benzoylchloride as the acylating agent. In addition to their excellent catalytic reactivities, the fly ash-based geopolymer catalysts provide a valuable approach of the 
utilisation of industrial wastes such as fly ash, the vast production of which is becoming a world-wide concern.

The geopolymer-based catalysts developed in this work are reusable without significant loss of reactivity and their catalytic performance is superior to other commonly used solid acid catalysts. The results presented in this thesis demonstrate a great potential for geopolymers as active candidates in the field of heterogeneous catalysis, representing as they do a new class of solid acids with highly desirable features such as catalytic efficiency as well as ecological friendliness, cost effectiveness and ease of synthesis. 
"To my dearest mother 'Itaf'

for all her dedication and sacrifice" 


\section{Research output}

\section{Publications}

- "Porous aluminosilicate inorganic polymers (geopolymers): a new class of environmentally benign heterogeneous solid acid catalysts” M. I. M. Alzeer, K. J. D. MacKenzie and R. A. Keyzers. (Applied Catalysis A: General 2016, 524, 173-181).

- "Facile synthesis of new hierarchical aluminosilicate inorganic polymer solid acids and their catalytic performance in alkylation reactions" M. I. M. Alzeer, K. J. D. MacKenzie and R. A. Keyzers. (Microporous and Mesoporous Materials 2017, 241, 316-325)

- "Friedel-Crafts alkylation of arenes over a new class of aluminosilicate inorganic polymer solid acids derived from fly ash” M. I. M. Alzeer, K. J. D. MacKenzie and R. A. Keyzers. (under review)

- "Fly ash-based aluminosilicate inorganic polymer solid acids and their catalytic applications in Friedel-Crafts acylation of aromatics" M. I. M. Alzeer, K. J. D. MacKenzie and R. A. Keyzers. (under review)

\section{Conferences}

- "Inorganic polymers (geopolymers) as novel catalysts for organic reactions" International Conference on Modern Materials and Technologies (CIMTEC 2014), Montecatini Terme, Italy. (invited speaker)

- "Geopolymers: a novel class of heterogeneous solid acid catalysts" Advanced Materials and Nanotechnology (AMN-7 2015), Nelson, New Zealand. 


\section{Acknowledgments}

This thesis would have not been achieved without the help and support I received from many individuals to whom I would like to extend my sincere thanks.

I would like to express my special gratitude to my advisor Prof. Kenneth MacKenzie for sharing his knowledge and expertise and providing the help and guidance that was essential for accomplishing this research. The kind support and commitment I received during the period of my Ph.D. research was tremendous, it was a privilege working under his supervision. I would also like to extend my thanks to Dr. Robert Keyzers, my secondary supervisor, who was always supportive and ready to help, it was great pleasure working with him.

I am indebted to Prof. Kate McGrath for the generous scholarship I was granted over the second half of my PhD which had enormously helped me pursing this thesis. Thanks are also extended to Victoria University of Wellington for granting me Curtis-Gordon Research Scholarship and Victoria Doctoral Submission Scholarship.

I would also like to thank the administration staff and laboratory technicians in the School of Physical and Chemical Sciences for being very friendly and helpful, particularly Jackie King and Teresa Gen. Special thanks go to David Flynn for his help with SEM and for collecting the TEM micrographs. I would also like to thank Dr. Mathew Cairns for his help with some of the lab instruments and for constructive conversations. Thanks are also extended to the staff in the Faculty of Science, particularly Patricia Stein for all the help and support.

I would like to express my love and extended thank to Maryke Barnard who has shared this experience with me from the start and been always by my side supportive and caring. I also express my gratitude to her parents Estelle and Johan Barnard for all the love and support they provided me, you guys have made New Zealand a home for me. 
My ultimate gratitude and appreciation goes to my family for their endless support and encouragement. I dedicate this thesis to my brothers Tawfeeq \& Mosa who funded my postgraduate studies and have always been very supportive and inspiring, my Dad and to my beloved Mother who has dedicated her life to us and has always been a typical model of a great mother.

Mohammad I.M. Alzeer 


\section{Content}

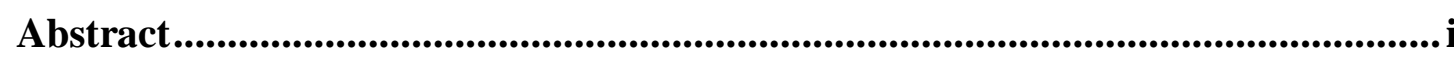

Research output ........................................................................................ iv

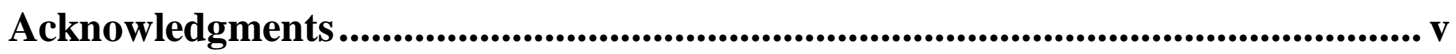

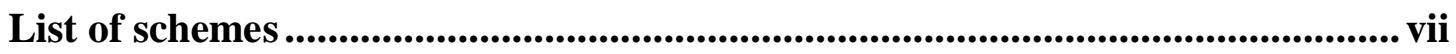

List of Figures .............................................................................................................. viii

List of Tables...................................................................................................................... $\mathrm{xV}$

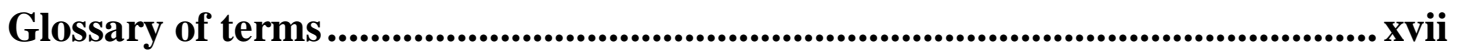

Chapter 1 Introduction and literature review .................................................... 1 -

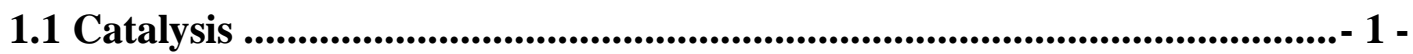

1.2 Heterogeneous Catalysis ........................................................................................ - 3 -

1.3 Solid Acids.................................................................................................. 3 -

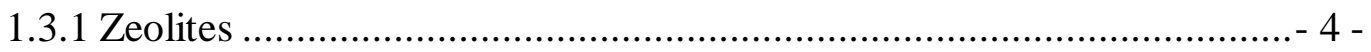

1.3.2 Mesoporous silicates and aluminosilicates ....................................... 7 -

1.3.3 Clays and clay-supported catalysts ............................................... 9 -

1.3.4 Oxides and mixed oxides based catalysts ........................................ 12 -

1.3.5 Heteropolyacids ......................................................................... 14 -

1.3.6 Amorphous silica-alumina (ASA) ............................................. 15 -

1.4. Scope of this thesis................................................................................................ 16 -

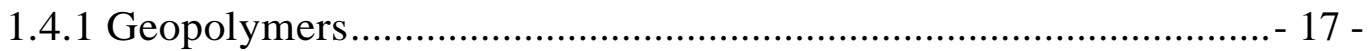

1.4.1 Geopolymers in heterogeneous catalysis........................................ 19 -

1.4.2 Novelty and research approaches................................................. 20 -

Chapter 2 Experimental .......................................................................... 21 -

2.1 Synthesis and catalysts preparation ........................................................ 21 - 
2.1.1 Geopolymer synthesis (the section was rearranged) .......................... 21 -

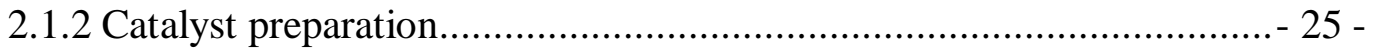

2.1.3 Post synthetic treatment ................................................................ 26 -

2.2 Catalyst characterisation .................................................................... 27 -

2.2.1 X-ray diffraction (XRD) .................................................................. 27 -

2.2.2 X-ray fluorescence (XRF) ........................................................... 29 -

$2.2 .3{ }^{27} \mathrm{Al}$ and ${ }^{29} \mathrm{Si}$ MAS NMR ............................................................. 29

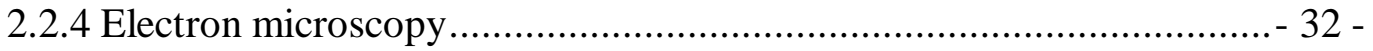

2.2.5 Fourier transform infrared spectroscopy (FTIR) ................................ 35 -

2.2.6 Surface acidity characterisation ..................................................... 36 -

2.2.7 Porosimetry measurements ........................................................... 39 -

2.2.8 Particle size distribution ................................................................ 45 -

2.3 Catalytic reactions.......................................................................... 46 -

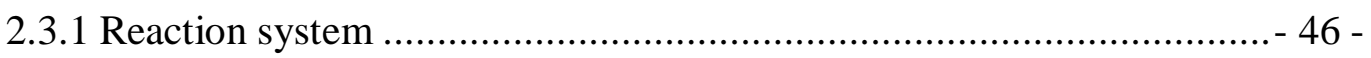

2.3.2 Analysis of the reaction outcomes .................................................. 46 -

2.3.3 Catalytic activity ......................................................................... 47 -

\section{Chapter 3 Activity of geopolymer catalysts in Beckmann rearrangement}

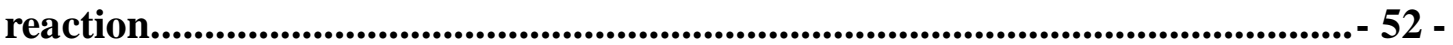

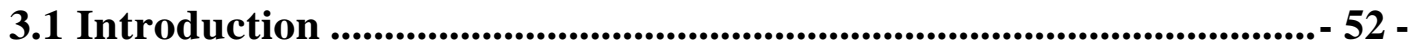

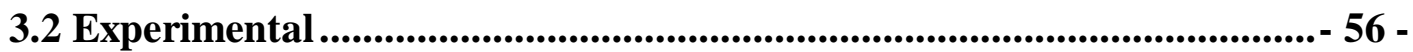

3.2.1 The catalyst preparation ........................................................... 56 -

3.2.2 Catalytic reaction ................................................................. 56 -

3.3 Results and discussion......................................................................... 57 -

3.3.1 Catalyst characteristics ….......................................................... 57 -

3.3.2 Generation of acid sites for Beckmann rearrangement ....................... 67 -

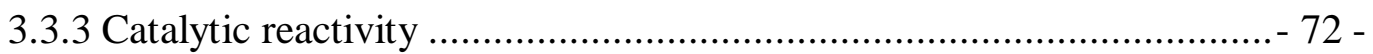

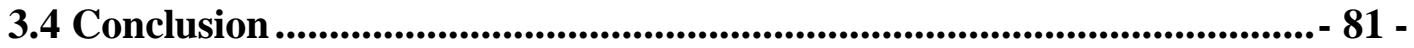

Chapter 4 Friedel-Crafts alkylation reactions catalysed over geopolymer

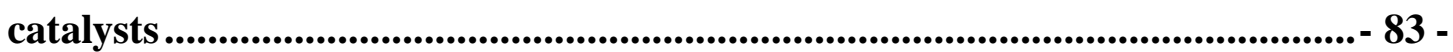

4.1 Introduction ........................................................................................... 83 - 
4.2 Experimental ........................................................................................................ 86 -

4.2.1 The catalyst preparation ................................................................. 86 -

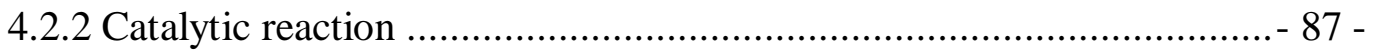

4.3 Results and discussion......................................................................................- 87 -

4.3.1 Catalysts characteristics................................................................... 87 -

4.3.2 Catalytic Reactivity ........................................................................ 93 -

4.4 Conclusion ……….................................................................................... 102 -

Chapter 5 Activity of fly ash-based geopolymer catalysts in alkylation reactions .103 -

5.1 Introduction ......................................................................................................... 103 -

5.1.1 Background to fly ash..................................................................- 104 -

5.1.2 Applications ...............................................................................- 106 -

5.2 Experimental .............................................................................................. 108 -

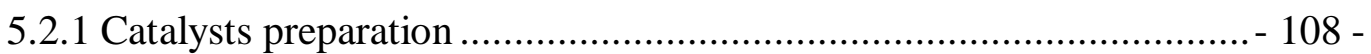

5.2.2 Catalytic reaction ....................................................................... 108 -

5.3 Results and discussion............................................................................ 108 -

5.3.1 Catalysts characteristics ............................................................................ 108 -

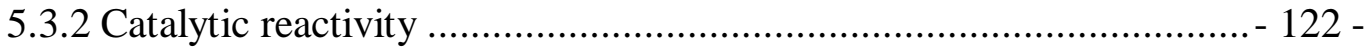

5.4 Conclusion ............................................................................................................ 135 -

Chapter 6 Reactivity of fly ash-based catalysts in Friedel-Crafts acylation reactions ................................................................................................................... 136 -

6.1 Introduction ............................................................................................................. - 136 -

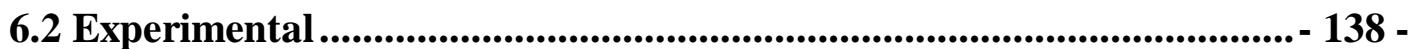

6.2.1 Catalysts preparation ..................................................................... 138 -

6.2.2 Catalytic reactions ................................................................... 138 -

6.3 Results and discussion...................................................................................... 139 -

6.4 Conclusion ......................................................................................................... 144 -

Chapter 7 Conclusion and future work ..................................................................... - 145 -

7.1 Conclusion ............................................................................................................. 145 - 


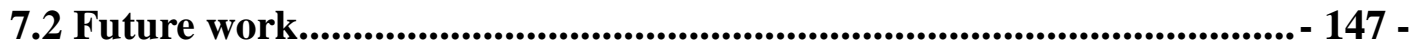

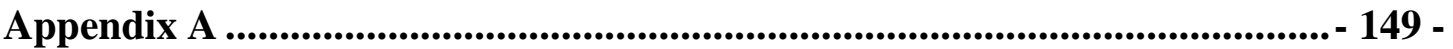

Bibliography ........................................................................................................... - 150 - 


\section{List of schemes}

Scheme 1.1 Illustration of a Bronsted acid site in zeolites (redrawn from ref. ${ }^{[19 b]}$ ). 5 Scheme 1.2 A comparison of the catalytic performance of micro- and mesoporous zeolite with MFI topology (redrawn from ref. ${ }^{[32]}$ ).......................................... 8 -

Scheme 1.3 Three different main building units of a geopolymer........................ 18 Scheme 4.1 Friedel-Crafts alkylation of benzene or substituted benzene with benzyl halide. - 84 -

Scheme 4.2 Monobenzylated products of Friedel-Crafts alkylation of several aromatics - 99 -

Scheme 6.1 Friedel-Crafts acylation of arenes with benzoyl halide as the acylating agent. 137 -

Scheme 6.2 The outcomes of the acylation reaction of different aromatics with BzoylCl. 140 - 


\section{List of Figures}

Figure 1.1 Zeolite frameworks; MFI e.g. ZSM-5 zeolite, and FAU e.g. Y zeolite [Database of Zeolite Structures (http://www.iza-structure.org/databases/)] $4-$

Figure 1.2 Conversion of methanol to olefins and other hydrocarbons over a zeolite catalyst (taken from ref. ${ }^{[25]}$ with permission). $6-$

Figure 1.3 Structural representation of MCM-41 (without surfactant), and MCM-48 (with surfactant) ${ }^{[29]}$ $-7-$

Figure 1.4 soft and hard-templating methods for mesoporous silicates synthesis (reproduced from ref. ${ }^{[34]}$ with permission). $-9-$

Figure 1.5 Representation of kaolinite and Smectite clays structure, showing the arrangements of silica and alumina layers (reproduced from ref. ${ }^{[38]}$ ). -10 -

Figure 1.6 Illustration of clay pillaring (reproduced from ref. ${ }^{[38]}$ ) $-11-$

Figure 1.7 Illustration of Bronsted acidity induced by coordinated Lewis acid site ${ }^{[48]}$. $-13-$

Figure 1.8 Illustration of the surface acidity of sulfated $\mathrm{ZrO}_{2}{ }^{[51]}$. $-13-$

Figure 1.9 phosphotungstate anion ${ }^{[55]}$ $-14-$

Figure 1.10 Schematic representation of the geopolymer framework - 17 -

Figure 2.1 Illustration of XRD theory $28-$

Figure 2.2 A representation of the Fourier transformation of FID to NMR spectrum ${ }^{[91]}$. $-30-$

Figure 2.3 (a) magic angle spinning probe and (b) ${ }^{27} \mathrm{Al} \mathrm{NMR}$ of a mixer of $\mathrm{Al}_{2} \mathrm{O}_{3}$ and $\mathrm{Y}_{2} \mathrm{O}$ measured for unspun (upper spectrum) and spun at the magic angle (bottom spectrum) ${ }^{[91]}$. The peaks marked with asterisks are spinning side bands. 31 - 
Figure 2.4 Illustration of some interactions between the primary electron beam and the sample in a scanning electron microscope (improved version from ${ }^{[95]}$ ). -33 -

Figure 2.5 Illustration of hydroxyl groups in different environments in silicates and aluminosilicates and their distinctive IR absorption. -37 -

Figure 2.6 Types of physisorption isotherms (adsorption-desorption) (a), and the hysteresis loops associated with physisorption isotherm type IV (b) ${ }^{[103]}$. -41 -

Figure 2.7 Stages of gas physisorption on a mesoporous solid. $-43-$

Figure 2.8 Illustration of the $t$-plot method. $-44-$

Figure 3.1 Industrial production of $\varepsilon$-caprolactam ${ }^{[122]}$ $-52-$

Figure 3.2 Reaction mechanism of Beckmann rearrangement of cyclohexanone oxime to $\varepsilon$-caprolactam (redrawn from ref. ${ }^{[134]}$ ). 54 -

Figure 3.3 Role of the solvent $(\mathrm{PhCN})$ in the 1,2 $\mathrm{H}$-shift during Beckmann rearrangement of cyclohexanone oxime to $\varepsilon$-caprolactam (edited from ref ${ }^{[129 a]}$ ). .- 55 Figure 3.4 Fig. 1. XRD traces of the halloysite clay and the geopolymers. (a) halloysite clay, (b) dehydroxylated halloysite clay, (c) K-hiSi, (d) K-N, (e) Na-N, (f) Na-hiSi, (g) Na-hiSi-Seq. (h: aluminium silicate hydroxide (halloysite); q: quartz; c: cristobalite; $\left.\mathrm{Na}_{2} \mathrm{CO}_{3} . \mathrm{H}_{2} \mathrm{O}\right)$. -58 -

Figure 3.5 A representative XRD traces for one of the prepared geopolymers after heating to $450{ }^{\circ} \mathrm{C}$ for $10 \mathrm{~min}$. (a) Na-hiSi-450 ${ }^{\circ} \mathrm{C}$, (b) Na-hiSi-Seq-450 ${ }^{\circ} \mathrm{C}$. (q: quartz; c: cristobalite; $\mathrm{Na}_{2} \mathrm{CO}_{3} . \mathrm{H}_{2} \mathrm{O}$ ) 59 -

Figure 3.6 ${ }^{27} \mathrm{Al}$ MAS NMR spectra of the as-synthesised geopolymer catalysts. (a) KhiSi, (b) K-N, (c) Na-N, (d) Na-hiSi, (e) Na-hiSi-Seq. $-60-$

Figure 3.7 ${ }^{29}$ Si MAS NMR spectra of the as-synthesised geopolymer catalysts. (a) KhiSi, (b) K-N, (c) Na-N, (d) Na-hiSi, (e) Na-hiSi-Seq. The asterisks denote spinning side bands. -61 -

Figure 3.8 FTIR spectra of the parent geopolymers. (a) K-hiSi, (b) K-N, (c) Na-N, (d) Na-hiSi. -62 -

Figure 3.9 SEM micrographs of the prepared geopolymers. (a) K-hiSi, (b) K-N, (c) NaN, (d) Na-hiSi. 63 - 
Figure 3.10 Particle size distribution of selected geopolymers before and after the sequential treatment $63-$

Figure 3.11 $\mathrm{N}_{2}$ adsorption-desorption isotherms of the geopolymer-based catalysts...$65-$

Figure 3.12 TEM micrographs of the parent geopolymers (a-d) and the sequentially treated geopolymers (e-h). (a) K-hiSi, (b) K-N, (c) Na-N, (d) and (e) Na-hiSi, (f) K-hiSi -Seq, (g) K-N-Seq, (h) Na-N-Seq, (i) and (j) Na-hiSi-Seq. $-66-$

Figure 3.13 FTIR spectra of (a) as-prepared Na-hiSi, (b) $\mathrm{NH}_{4}{ }^{+}$-ion-exchanged Na-hiSi, (c) after thermal treatment at $450{ }^{\circ} \mathrm{C}$ for $10 \mathrm{~min}$, (d) Na-hiSi-Seq after thermal treatment at $450{ }^{\circ} \mathrm{C}$ for $10 \mathrm{~min}$, (e) Na-hiSi after thermal treatment at $550{ }^{\circ} \mathrm{C}$ for $10 \mathrm{~min} . . . .-67$ -

Figure 3.14 ${ }^{27} \mathrm{Al}$ MAS NMR of a selected geopolymer after thermal treatment at different temperature. (a) Na-hiSi as-synthesised, (b) Na-hiSi after thermal treatment at $450{ }^{\circ} \mathrm{C}$ for $10 \mathrm{~min}$, (c) Na-hiSi after thermal treatment at $550{ }^{\circ} \mathrm{C}$ for $10 \mathrm{~min}$. $69-$

Figure 3.15 FTIR spectra of the activated geopolymer before and after adsorption of pyridine; (a) Na-hiSi without pyridine, (b) Na-hiSi-pyridine, (c) Na-hiSi-Seq without pyridine, (d) Na-hiSi-Seq-pyridine. $-70-$

Figure 3.16 TGA traces of pyridine desorption. (a) sample Na-hiSi, (b) Na-hiSi-Seq. $71-$

Figure 3.17 ${ }^{27} \mathrm{Al}$ MAS NMR spectra of Na-hiSi (a) and Na-hiSi-Seq (b) both heated to $450{ }^{\circ} \mathrm{C}$ for $10 \mathrm{~min}$. $-72-$

Figure 3.18 Catalytic activity of the various geopolymer-based catalysts compared with zeolite catalysts in the Beckmann rearrangement of cyclohexanone oxime to $\varepsilon$ caprolactam. (a) degree of conversion of the oxime, (b) selectivity for the caprolactam product. Reaction conditions: solvent $20 \mathrm{ml} \mathrm{PhCN} ; 0.1 \mathrm{~g}$ catalyst; $0.1 \mathrm{~g}$ oxime; $\mathrm{T}=130$ ${ }^{\circ} \mathrm{C}$. $-74-$

Figure 3.19 Catalytic performance of Na-hiSi-Seq as a function of reaction time. Reaction conditions: solvent $20 \mathrm{ml} \mathrm{PhCN} ; 0.1$ g catalyst; $0.1 \mathrm{~g}$ oxime; $\mathrm{T}=130{ }^{\circ} \mathrm{C} .-76$ 
Figure 3.20 The influence of the reaction temperature (a), and the oxime: catalyst wt. $\%$ (b) on the Beckmann rearrangement reaction. The catalyst is Na-hiSi-Seq, solvent $\mathrm{PhCN}$. - 77 -

Figure 3.21 Reusability of Na-hiSi-Seq over five reaction cycles. Reaction conditions; 1:1:200 oxime: catalyst: solvent wt. $\% ; \mathrm{T}=130{ }^{\circ} \mathrm{C} ; \mathrm{t}=24 \mathrm{hr}$. $-78-$

Figure 3.22 XRD traces of (a) fresh Na-hiSi-Seq catalyst and (b) the recovered catalyst after five reaction cycles. - 79 -

Figure 3.23 FTIR spectra of (a) fresh Na-hiSi-Seq catalyst, (b) the recovered catalyst after five reaction cycles, and (c) the recovered catalyst after heating to $450{ }^{\circ} \mathrm{C} . . .-79$ -

Figure 4.1 Several diaryalkanes and their biological activities ${ }^{[143]}$. $85-$

Figure 4.2 A mechanism of Friedel-Crafts alkylation of benzene or substituted benzene with benzylhalide as alkylating agent. $86-$

Figure 4.3 XRD traces of geopolymers thermally treated to $550{ }^{\circ} \mathrm{C}$ for $15 \mathrm{~min}$. (a) KhiSi, (b) K-N, (c) Na-N, (d) Na-hiSi, (e) Na-hiSi-Seq. (q: quartz; c: cristobalite; $\mathrm{Na}_{2} \mathrm{CO}_{3} . \mathrm{H}_{2} \mathrm{O}$ ). - 88 -

Figure 4.4 IR spectra of adsorbed pyridine over Na-hiSi-550 ${ }^{\circ} \mathrm{C}$ with and without sequential treatment; (a) Na-hiSi-550 ${ }^{\circ} \mathrm{C}$-background, (b) Na-hiSi-550 ${ }^{\circ} \mathrm{C}$-pyridine, (c) Na-hiSi-Seq-550 ${ }^{\circ} \mathrm{C}$-background, (d) Na-hiSi-Seq-550 ${ }^{\circ} \mathrm{C}$-pyridine. $89-$

Figure 4.5 TGA profiles of pyridine degradation of representative catalysts that were heated to $550{ }^{\circ} \mathrm{C}$ for $15 \mathrm{~min}$. before and after sequential treatment. (a) Na-hiSi, (b) NahiSi-Seq. $-90-$

Figure 4.6 FTIR spectra in the $\mathrm{OH}$ region of $\mathrm{NH}_{4}{ }^{+}$-ion exchanged geopolymers with and without sequential treatment, after heating at different temperatures. (a) Na-hiSi$450{ }^{\circ} \mathrm{C}$, (b) Na-hiSi-550 ${ }^{\circ} \mathrm{C}$, (c) Na-hiSi-Seq $550{ }^{\circ} \mathrm{C}$ - 91 -

Figure 4.7 Representative ${ }^{27} \mathrm{Al}$ MAS NMR spectra of geopolymer catalysts with different compositions at different stages of post-synthetic treatments. (a-c) Na-N; (a) Na-N parent, (b) Na-N-DA, (c) Na-N-Seq, (d-f) Na-hiSi; (d) Na-hiSi parent, (e) NahiSi-DA, (f) Na-hiSi-Seq. - 92 -

Figure 4.8 ${ }^{27} \mathrm{Al}$ MAS NMR spectra of heated geopolymer catalysts heated at $550{ }^{\circ} \mathrm{C}$ without sequential treatment (a-d); (a) K-hiSi, (b) K-N, (c) Na-N, (d) Na-hiSi, and with 
sequential treatment (e-h); (e) K-hiSi-Seq, (f) K-N-Seq, (g) Na-N-Seq, (h) Na-hiSi-Seq. $-93-$

Figure 4.9 Catalytic reactivity of the various geopolymer catalysts in alkylation of toluene with $\mathrm{BzCl}$. Reaction conditions: $13.0 \mathrm{ml}$ toluene; $1.0 \mathrm{ml} \mathrm{BzCl} ; 0.1 \mathrm{~g}$ catalyst; $\mathrm{T}$ $110^{\circ} \mathrm{C}$. $-94-$

Figure 4.10. Langmuir-Hinshelwood pseudo-first-order kinetic model and for alkylation of toluene with $\mathrm{BzCl}$ over $\mathrm{Na}$-hiSi-Seq. $-96-$

Figure 4.11 Arrhenius plot for alkylation of toluene with $\mathrm{BzCl}$ over Na-hiSi-Seq. $-96-$

Figure 4.12 The influence of the substrate: catalyst wt. \% on the alkylation of toluene with $\mathrm{BzCl}$ over Na-hiSi-Seq catalyst. $\mathrm{T}=110^{\circ} \mathrm{C}$ 97 -

Figure 4.13 Catalytic reactivity of Na-hiSi-Seq in alkylation of mesitylene, p-xylene, anisole, and toluene with $\mathrm{BzCl}$. Reaction conditions: $13.0 \mathrm{ml}$ of each aromatic; $1.0 \mathrm{ml}$ $\mathrm{BzCl} ; 0.1 \mathrm{~g}$ catalyst; $\mathrm{T}=110^{\circ} \mathrm{C}$. - $99-$

Figure 4.14 Reusability of Na-hiSi-Seq catalyst in alkylation of toluene with $\mathrm{BzCl}$. Reaction conditions; 130:10:1 Toluene: $\mathrm{BzCl}$ : catalyst wt. \%; T $110^{\circ} \mathrm{C}$. $-101-$

Figure 4.15 XRD traces of (a) fresh Na-hiSi-Seq catalyst and (b) the recovered catalyst after five reaction cycles. $-101-$

Figure 5.1 XRD of geopolymers prepared from different fly ashes. (a) raw fly ash, (b) fly ash-based geopolymer "as-synthesised", (c) $\mathrm{NH}_{4}{ }^{+}$-form of the geopolymer, (d) $\mathrm{NH}_{4}{ }^{+}$-geopolymer after heating to $550{ }^{\circ} \mathrm{C}$. - 109 -

Figure 5.2 FTIR spectra of geopolymers prepared from different fly ashes. (a) raw fly ash, (b) fly ash-based geopolymer "as-synthesised", (c) $\mathrm{NH}_{4}{ }^{+}$-form of the geopolymer, (d) $\mathrm{NH}_{4}{ }^{+}$-geopolymer after heating to $550{ }^{\circ} \mathrm{C}$. - 111 -

Figure 5.3 SEM micrographs the raw fly ashes (a-d) and the prepared geopolymers (eh). (a) Hyrock, (b) Huntly, (c) Mt. piper, (d) G.S., (e) Geo-Hyrock, (f) Geo-Huntly, (g) Geo-Mt. piper, (h) Geo-G.S. - 113 -

Figure 5.4 Particle size distribution of the raw fly ashes (a) and the corresponding fly ash-based catalysts (b). 114 -

Figure 5.5 $\mathrm{N}_{2}$ adsorption-desorption isotherms of Geo-Huntly catalyst $-115-$ 
Figure 5.6 TEM micrographs of the fly ash-based geopolymer catalysts. (a) GeoHyrock, (b) Geo-Huntly, (c) Geo-Mt. piper, (d) Geo-G.S................................. 116 -

Figure 5.7 TGA profiles of pyridine desorption from the fly ash-based catalysts. The dashed line is the background and the solid line represents the pyridine desorption. (a) Geo-Hyrock, (b) Geo-Huntly, (c) Geo-Mt. piper, (d) Geo-G.S. - 117 -

Figure 5.8 FTIR spectra of the fly ash-based catalysts without pyridine (dashed line), and with desorbed pyridine (solid line). (a) Geo-Hyrock, (b) Geo-Huntly, (c) Geo-Mt. piper, (d) Geo-G.S. ........................................................................ 118 -

Figure 5.9 EDS analysis of Geo-Hyrock catalyst.......................................... 119 -

Figure 5.10 Element distribution maps of Geo-Hyrock catalyst....................... 120 -

Figure 5.11 High magnification SEM images of specific spots containing Fe species. Each image (a-d) represents the red circled spots identified at the top two source images. $-121-$

Figure 5.12 Catalytic reactivity of Geo-Hyrock in alkylation of several aromatics. (a) conversion of $\mathrm{BzCl}$, (b) selectivity to monobenzylated products. Reaction conditions; $13.0 \mathrm{ml}$ of each aromatic; $1.0 \mathrm{ml} \mathrm{BzCl} ; 0.1 \mathrm{~g}$ catalyst; $\mathrm{T}=80{ }^{\circ} \mathrm{C}$ $-122-$

Figure 5.13 Catalytic reactivity of raw Hyrock fly ash (a) and H-Y zeolite (b) in the alkylation of various aromatics with $\mathrm{BzCl}$. Reaction conditions; $13.0 \mathrm{ml}$ of each aromatic; $1.0 \mathrm{ml} \mathrm{BzCl} ; 0.1 \mathrm{~g}$ catalyst; $\mathrm{T}=80^{\circ} \mathrm{C}$. $123-$

Figure 5.14 Langmuir-Hinshelwood pseudo-first-order kinetic model for the alkylation reactions of benzene, toluene, anisole, p-xylene, and mesitylene over the Geo-Hyrock catalyst. $-124-$

Figure 5.15 Catalytic reactivities of various fly ash-based catalysts in the alkylation of several aromatics. (a) and (b) Geo-Huntly, (c) and (d) Geo-G.S., (e) Geo-Mt. Piper. Reaction conditions; $13.0 \mathrm{ml}$ of each aromatic; $1.0 \mathrm{ml} \mathrm{BzCl} ; 0.1 \mathrm{~g}$ catalyst; $\mathrm{T}=80{ }^{\circ} \mathrm{C}$. $-126-$

Figure 5.16 Proposed redox mechanism of Fe-containing catalysts in alkylation of benzene with $\mathrm{BzCl}$ (redrawn from ref. ${ }^{[181]}$ )..... $128-$ 
Figure 5.17 Alkylation of various aromatics with $\mathrm{BzCl}$ carried over Geo-Hyrock catalyst at different temperatures. (a) $80{ }^{\circ} \mathrm{C}$, (b) $90^{\circ} \mathrm{C}$, (c) $100{ }^{\circ} \mathrm{C}$, (d) $110^{\circ} \mathrm{C}$. Aromatic: $\mathrm{BzCl}$ wt. $\%=13 ; 0.1 \mathrm{~g}$ cat. $-130-$

Figure 5.18 Arrhenius plot for the alkylation of toluene and p-xylene with $\mathrm{BzCl}$ over Geo-Hyrock catalyst. $131-$

Figure 5.19 The influence of the catalyst: substrate wt.\% on the outcomes of the alkylation reaction of toluene with $\mathrm{BzCl}$ over Geo-Hyrock catalyst. (a) at $80{ }^{\circ} \mathrm{C}$, (b) at $90{ }^{\circ} \mathrm{C}$. aromatic: $\mathrm{BzCl}$ wt. $\%=13$. $-131-$

Figure 5.20 The reusability of Geo-Hyrock in alkylation of toluene with $\mathrm{BzCl}$. Reaction conditions; toluene: $\mathrm{BzCl}$ wt. $\%=13 ; \mathrm{BzCl}$ : catalysts wt. $\%=10 ; \mathrm{t}=90{ }^{\circ} \mathrm{C}$. .133 -

Figure 5.21 FTIR spectra of a fresh Geo-Hyrock catalyst prior use (a), and the reused catalyst after five reaction cycles (b). - 134 -

Figure 5.22 TGA of a fresh Geo-Hyrock catalyst prior use (dashed red line), and the reused catalyst after five reaction cycles (solid black line). $-134-$

Figure 6.1 Catalytic reactivity of Geo-Huntly in the acylation of several aromatics with BzoylCl. Reaction conditions; $13 \mathrm{ml}$ aromatic, $1 \mathrm{ml} \mathrm{BzoylCl,} 0.1 \mathrm{~g}$ catalyst; $\mathrm{T}=130{ }^{\circ} \mathrm{C}$ for all aromatics except toluene $110^{\circ} \mathrm{C}$. $139-$

Figure 6.2 Langmuir-Hinshelwood pseudo-first-order kinetic model for the acylation of several aromatics over the Geo-Huntly catalyst. Reaction conditions as described for Figure 6.1 $-141-$

Figure 6.3 Catalytic reactivity of Geo-Huntly catalyst (a) and Geo-Hyrock catalyst (b) in the Friedel-Crafts acylation of anisole with BzoylCl at different reaction temperatures. Reaction conditions; $13 \mathrm{ml}$ anisole, $1 \mathrm{ml}$ BzoylCl, 0.1 g catalyst. .- 142 Figure 6.4 Influence of the catalyst: substrate wt.\% on the acylation reaction of anisole with BzoylCl over Geo-Huntly catalyst. (a) at $120{ }^{\circ} \mathrm{C}$, (b) at $130{ }^{\circ} \mathrm{C}$. Reaction conditions; anisole: BzoylCl wt.\% 13 $143-$ 


\section{List of Tables}

Table 2.1 Chemical composition of the halloysite clay and the fly ashes ${ }^{\text {a }}$ $-22-$

Table 2.2 weights in grams (g) of all the components used in the geopolymers synthesis - 23 -

Table 2.3 Chemical composition of the synthesised clay based geopolymers a ${ }^{\text {a..... }} 24$ -

Table 2.4 Molar compositions of the synthesised fly ash-based geopolymers a ....- 25 -

Table 3.1 Textural and acidic properties of the various geopolymer-based catalysts...64 -

Table 3.2 Different solvents used in Beckmann rearrangement reaction $-73-$

Table 3.3 Comparison of the catalytic performance of geopolymer-based catalysts with other catalysts for the liquid phase Beckmann rearrangement of cyclohexanone oxime to $\varepsilon$-caprolactam. Reaction conditions: solvent $20 \mathrm{ml} \mathrm{PhCN}$; $0.1 \mathrm{~g}$ catalyst; $0.1 \mathrm{~g}$ oxime; $\mathrm{T}=130{ }^{\circ} \mathrm{C} ; \mathrm{t}=5 \mathrm{hr}$. 80 -

Table 3.4 Reaction kinetics of Beckmann rearrangement of cyclohexanone oxime measured at $5 \mathrm{hr}$. reaction time over selected geopolymers and zeolites. $-81-$

Table 4.1 Total acid amount of the $\mathrm{NH}_{4}{ }^{+}$-ion exchanged geopolymers after heating to $550{ }^{\circ} \mathrm{C}$. - 89 -

Table 4.2. Catalytic reactivity of several sequentially treated geopolymer-based catalysts in alkylation of toluene with benzylchloride ${ }^{\text {a }}$ -95 -

Table 4.3 Physical and chemical properties of the catalyst, solvent and reactant in alkylation of toluene with $\mathrm{BzCl}$ over $\mathrm{Na}-\mathrm{hiSi}$-Seq ${ }^{\mathrm{a}}$. $-98-$

Table 4.4 Catalytic activity of Na-hiSi-Seq in the alkylation of several aromatics at 1 hr. reaction time ${ }^{\mathrm{a}}$. $100-$

Table 5.1 Global production and utilisation of CCPs by country or region in $2010^{[159]}$. - 104 -

Table 5.2 Elemental composition (wt. \%) of fly ashes produced from the combustion of different coals ${ }^{[162]}$. 105 - 
Table 5.3 Metal oxides (wt.\%) of the as-synthesised and ion exchanged fly ash-based geopolymers ${ }^{\text {a }}$ $112-$

Table 5.4 Physical and chemical properties of the fly ash-based catalysts. $115-$

Table 5.5 Kinetic parameters of the catalytic reactivity of Geo-Hyrock in the alkylation of the various aromatics with $\mathrm{BzCl}$ after 30 min. reaction time ${ }^{\mathrm{a}}$. $125-$ Table 5.6 Comparison of the catalytic reactivity of the various catalysts in the alkylation of toluene with $\mathrm{BzCl}$ at 30 min. reaction time ${ }^{\mathrm{a}}$ $-127-$

Table 5.7 Catalytic reactivity of fly ash-based catalysts with other reported $\mathrm{Fe}$ containing solid catalysts in the alkylation of benzene with $\mathrm{BzCl}$ under similar reaction conditions. 129

Table 5.8 Madon-Boudart test for the mass and heat transfer limitations. $-132-$

Table 6.1 Catalytic reactivity of Geo-Huntly in the acylation of several aromatics with BzoylCl as the acylating agent ${ }^{\mathrm{a}}$. $140-$ 


\section{Glossary of terms}

\begin{tabular}{|c|c|}
\hline BET & Brunauer-Emmett-Teller \\
\hline BJH & Barrett-Joyner-Halenda \\
\hline $\mathrm{B}_{\mathrm{o}}$ & External magnetic field \\
\hline BSE & Backscattered electron images \\
\hline $\mathrm{BzCl}$ & Benzylchloride \\
\hline BzoylCl & Benzoylchloride \\
\hline BzTol & Benzyltoluene \\
\hline $\mathrm{Ca}$ & Concentration \\
\hline CCPs & Coal combustion products \\
\hline $\mathrm{Cf}$ & Concentration at a given time \\
\hline $\mathrm{Ci}$ & Concentration originally added \\
\hline DA & Dealumination \\
\hline $\mathrm{Db}$ & Bulk diffusivity \\
\hline Deff & Effective diffusivity \\
\hline DPM & Diphenylmethane \\
\hline$D_{\text {pore }}$ & Pore diameter \\
\hline $\mathrm{Ea}$ & Activation energy \\
\hline EDS & Energy dispersive X-ray fluorescence spectroscopy \\
\hline EFAl & Extra framework aluminium \\
\hline FAl & Framework aluminium \\
\hline FID & Free induction decay \\
\hline FTIR & Fourier transform infrared spectroscopy \\
\hline Geo-G.S. & Geopolymer made from Gladstone fly ash \\
\hline Geo-Mt. piper & Geopolymer made from Mount piper fly ash \\
\hline $\mathrm{H}_{\mathrm{o}}$ & Hammett acidity function \\
\hline
\end{tabular}




\begin{tabular}{|c|c|}
\hline Huntly-Geo & Geopolymer made from Huntly fly ash \\
\hline Hyrock-Geo & Geopolymer made from Hyrock fly ash \\
\hline $\mathrm{k}$ & reaction constant \\
\hline K-hiSi & Potassium -geopolymer with high silicon content \\
\hline $\mathrm{K}-\mathrm{N}$ & Potassium-geopolymer with normal composition \\
\hline Lf & Larmor frequency \\
\hline LOI & Loss on ignition \\
\hline MAS NMR & Magic angle spinning nuclear magnetic resonance \\
\hline Mt & Million metric tonne \\
\hline$\eta$ & Viscosity \\
\hline Na-hiSi & Sodium-geopolymer with high silicon content \\
\hline $\mathrm{Na}-\mathrm{N}$ & Sodium-geopolymer with normal composition \\
\hline OSDA & Organic structural directing agents \\
\hline $\mathrm{P}$ & Ternan constant \\
\hline$p / p^{o}$ & Relative pressure \\
\hline r pore & Catalyst pore radius \\
\hline $\mathrm{ra}$ & Reaction rate \\
\hline $\mathrm{rf}$ & radio frequency \\
\hline $\mathrm{Rp}$ & Catalyst particle radius \\
\hline$S$ & Surface area \\
\hline SE & Standard error \\
\hline SEM & Scanning electron microscopy \\
\hline Seq & Sequential treatment \\
\hline STEM & Scanning transmission electron microscopy \\
\hline STP & Standard temperature and pressure \\
\hline TEM & Transmission electron microscopy \\
\hline TGA & Thermal gravimetric analysis \\
\hline TOF & Turn over frequency \\
\hline TON & Turn over number \\
\hline TPD & Temperature programmed desorption \\
\hline
\end{tabular}




$\begin{array}{ll}V & \text { Pore volume } \\ \text { V } & \text { Molar volume } \\ \text { XRD } & \text { X-ray diffraction } \\ \text { XRF } & \text { X-ray florescence } \\ \chi & \text { Association constant } \\ \phi & \text { Thiele modulus } \\ \phi_{\text {W.P. }} & \text { Weisz-Prater criterion } \\ \boldsymbol{\eta} & \text { Effectiveness factor }\end{array}$





\section{Chapter 1}

\section{Introduction and literature review}

\subsection{Catalysis}

Catalysis is one of the most important fields in chemistry in which around $90 \%$ of the chemical industries involve catalysis in at least one step of their reactions ${ }^{[1]}$. It is a chemical process where a substance (the catalyst) influences a reaction rate and thus controls the path of the reaction without being itself consumed. Catalysis plays a crucial role in the production of pharmaceuticals, fine chemicals and petrochemicals. Moreover, increased environmental awareness makes catalysis even more important as it limits the production of undesirable by-products, thus decreasing industrial wastes and producing energy-saving benefits.

The term catalysis was first coined by Berzelius in 1835 based on his analysis of the role of sulfuric acid on the conversion of ethanol to ether, the reaction devised by Eilhard Mitscherlich ${ }^{[2]}$. In fact, catalysed processes were carried out more than a century earlier, in the 16th and 17th centuries, in the fermentation of sugar to produce alcoholic beverages and in the production of soap by fat hydrolysis ${ }^{[3]}$. In addition, the synthesis of sulfuric acid by the oxidation of sulfur dioxide to the trioxide using nitrogen oxide, the "lead chamber process", was first achieved in around $1750{ }^{[4]}$.

Berzelius defined catalysis as "a phenomenon that allows a reaction to occur faster when non-reactive species are present". In the $19^{\text {th }}$ Century, Mitscherlich introduced the term "contact catalysis" by studying the acceleration of reactions by solids. In 1895 Ostwald described a catalyst as "a substance that accelerates or retards a reaction", 
and received the 1909 Nobel prize for his work. In 1912, another Nobel prize winner, Paul Sabatier, introduced the "Sabatier principle" which states that the adsorption energy of a reactant adsorbed on the surface of a catalyst should be strong enough to cause activation, but not so strong as to inhibit product desorption. A very important concept, that of "active sites", is still used today in heterogeneous catalysis and was introduced by Taylor in 1925. This states that a chemical reaction does not occur on the whole surface of a catalyst but rather on specific active sites on the surface of the catalyst. Further details of the history and subsequent developments of catalysis have been extensively reviewed elsewhere ${ }^{[2-3,5]}$.

The first industrial breakthrough in catalysis was the production of ammonia from hydrogen and nitrogen under high pressure by Haber and Nernst in 1908, using osmium as the catalyst ${ }^{[3]}$. Thus, catalysis has played a vital role in the commercialisation of $\mathrm{NH}_{3}$ production and other chemicals that are essential in such diverse fields as energy, industry, the environment, and life sciences. Consequently, the turnover of the industrial production of catalysts reached 33.5 billion dollars in $2014^{[6]}$.

Catalysis can be classified in three different categories; heterogeneous, homogeneous and bio or enzymatic catalysis. The latter occurs either naturally in vivo, where proteins or RNA molecules act as catalysts, or in vitro using enzyme catalysts ${ }^{[7]}$. An example of this type of catalysis is the synthesis of polysaccharides, in which hydrolases catalyse the formation of glycosidic linkages between glycosyl molecules ${ }^{\text {[7a] }}$. Homogeneous catalysis, by definition, describes the type of catalysis where the substrate and the catalyst are in the same phase, often the liquid phase. Lewis acids, organic and inorganic bases and organometallic complexes all are examples of homogeneous catalysts. A common example of such a catalytic system is the hydroformylation of olefins to give the corresponding aldehydes using Co and Rh carbonyls ${ }^{[8]}$.

By contrast, in heterogeneous catalysis, the substrate is most often in the gas or liquid phase and the catalyst is in the solid phase. Although homogeneous catalysts are highly reactive, heterogeneous catalysis is often preferable in industrial processes, in which $\sim 90 \%$ of the catalytic reactions are carried out using heterogeneous catalysts ${ }^{[9]}$. The present work has focussed on heterogeneous catalysts, particularly solid acids. 


\subsection{Heterogeneous Catalysis}

Recent increased environmental awareness and the stimulus of greener chemistry has driven the rapid development of heterogeneous catalysts for a wide range of organic synthesis applications ${ }^{[10]}$. The separation of the homogenous catalysts from reaction mixtures remains their main limitation, adding to the cost and generating large amounts of industrial wastes. For example, when $\mathrm{AlCl}_{3}$ is used as a homogeneous acid catalyst, it is separated from the reaction mixture by breaking down the catalyst-product complex using water (water quenching), generating a large amount of acidic aluminium hydroxide $\left(\mathrm{Al}(\mathrm{OH})_{3}\right)$ as waste ${ }^{[11]}$. In addition, some homogeneous catalysts are toxic and corrosive; these include $\mathrm{AlCl}_{3}, \mathrm{BF}_{3}, \mathrm{H}_{2} \mathrm{SO}_{4}$ or liquid $\mathrm{HF}$ which are difficult to handle and dispose of and are not reusable. By contrast, solid catalysts are reusable, easy to separate from the reaction mixture and usually generate less by-products ${ }^{[12]}$.

Different types of solid catalysts have been developed in the last few decades. These include solid acids, bases, acid-base bifunctional and redox catalysts. Of these materials, solid acids are the most commonly used in industry, particularly in petroleum refining and cracking processes ${ }^{[13]}$. An overview of the heterogeneous solid acid catalysts is presented below.

\subsection{Solid Acids}

Since the early 1930s, solid acids with different acid strengths have been used in industry, the first of which were clays, followed by amorphous silica-alumina and then by zeolites. Unlike homogenous catalysis or bio-catalysis, there is a lack of fundamental understanding, on a molecular level, of the mechanisms associated with heterogeneous catalysis. This remains the main challenge in heterogeneous catalysis, and is required to understand the structure-activity relationship and thus design more refined and predictable heterogeneous catalysts ${ }^{[9,14]}$. The active sites in solid acids are usually described as Bronsted or Lewis acid sites. The nature of these active sites has been studied in terms of their strength, density and location. Typically, this has been investigated by spectroscopic analysis such as FT-IR, solid state MAS NMR, and adsorption experiments using probe molecules. The strength of these sites, and hence their catalytic activity is affected by their environment, which varies in different solids, 
depending on their surface structures, chemical composition, electronic effects, etc. ${ }^{[5]}$. What follows is an overview of the most commonly used solid acid catalysts.

\subsubsection{Zeolites}

Zeolites are the most common solid acids, being extensively used in oil refining and the petrochemicals industry since $1962^{[15]}$. These are solid crystalline microporous aluminosilicate inorganic polymers with three-dimensional frameworks forming channels and cages of up to $1 \mathrm{~nm}$ in diameter, consisting of $\mathrm{SiO}_{4}$ and $\left(\mathrm{AlO}_{4}\right)^{-}$tetrahedral units with ion-exchangeable cations to compensate for the negative charge on the $\left(\mathrm{AlO}_{4}\right)^{-}$tetrahedra ${ }^{[16]}$.

Zeolites are conventionally synthesised via hydrothermal processes at a temperature of $80-200{ }^{\circ} \mathrm{C}$, using inorganic or organic structural directing agents (OSDAs) in alkaline or fluoride media ${ }^{[17]}$. These templates are usually removed by heating at high temperatures, which increases the environmental burden, especially in view of the large-scale production of zeolites. The synthesis usually is carried out in an autoclave at high pressure for up to 20 days ${ }^{[18]}$. In the last few years, however, studies have suggested alternative routes to synthesise zeolites in a more environmentally friendly manner ${ }^{[17-18]}$.

Zeolites exhibit remarkable features compared with other solid acids, such as high surface areas, highly crystalline structures with well-defined pores and cavities (Figure 1.1), which result in shape-selectivity, and strong acidity ${ }^{[15]}$.

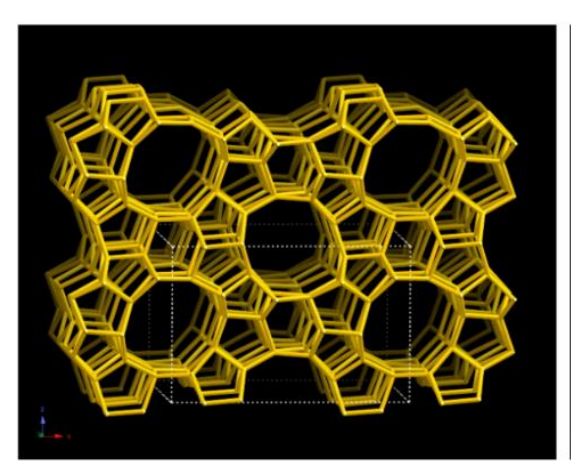

MFI Framework

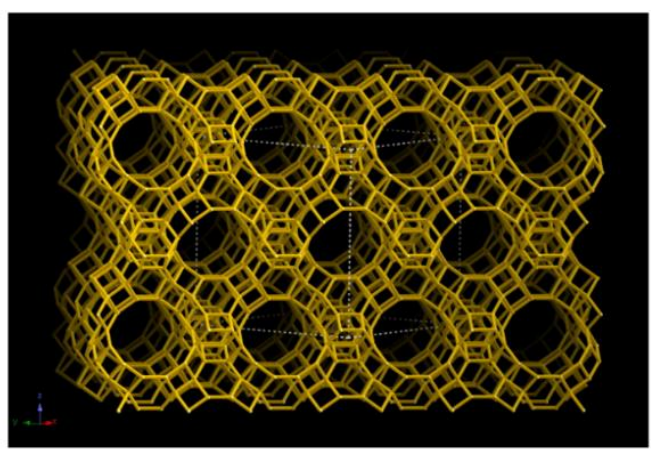

FAU Framework

Figure 1.1 Zeolite frameworks; MFI e.g. ZSM-5 zeolite, and FAU e.g. Y zeolite [Database of Zeolite Structures (http://www.iza-structure.org/databases/)]. 
The acidity of zeolites and other aluminosilicates is in the form of Bronsted and/or Lewis acid sites. Bronsted acid sites can be generated in the zeolite by ion-exchanging with $\mathrm{NH}_{4}{ }^{+}$followed by degradation of the ammonia by thermal treatment (usually around $500^{\circ} \mathrm{C}$ ) to form the $\mathrm{H}$-form of the zeolite. Literature reports agree that the origin of the Bronsted acid sites in zeolites arises from the bridging hydroxyl groups where the oxygen supporting the acidic hydrogen is associated with $\mathrm{Al}$ and $\mathrm{Si}$, as shown in scheme $1.1^{[19]}$. According to Pines Theory ${ }^{[20]}$, the acid strength of a given site is increased when the number of $\mathrm{Al}$ atoms in the next-nearest-neighbour (NNN) positions, indicated as red square in Scheme 1.1, decreased. Thus, acidic sites with 0 NNN Al possess the highest acidity.

Scheme 1.1 Illustration of a Bronsted acid site in zeolites (redrawn from ref. ${ }^{[19 b]}$ ).

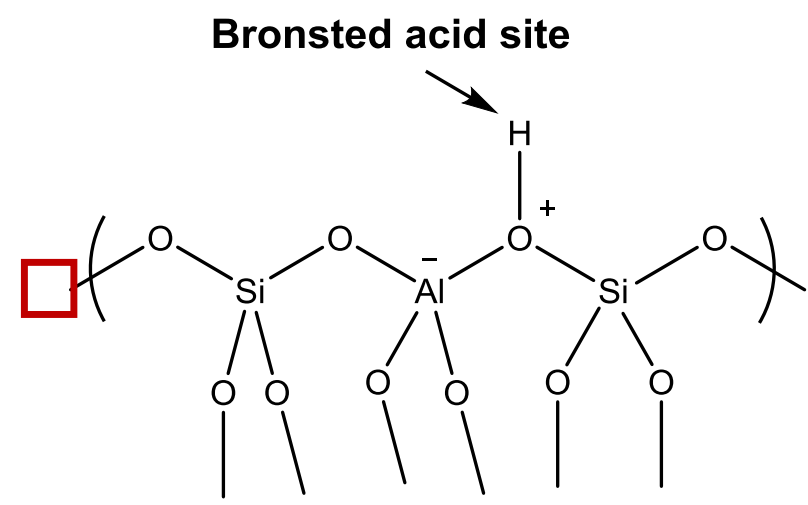

By contrast, the exact nature of Lewis acidic sites in the crystalline aluminosilicates (zeolites) is not clear at present. Generally, Lewis acidity is believed to be caused by extra-framework $\mathrm{Al}$ (EFAl.), 5- and 6-coordinated Al. These EFAl are generated by thermal and/or acidic treatment during the preparation of the catalyst, in which the tetrahedral $\mathrm{Al}$ is transformed to penta- and octahedral $\mathrm{Al}{ }^{[21]}$. In addition, tetrahedral and tri-coordinated framework $\mathrm{Al}$ can also act as Lewis acidic sites ${ }^{[22]}$.

Due to their special features, zeolites currently play a major role as industrial catalysts, mainly in the petrochemical industries. Examples of zeolite catalytic applications are numerous, and include the cracking of hydrocarbons over zeolite X, Y or ZSM-5 and the isomerisation of o-xylene to p-xylene, the precursor for terephthalic acid, over $\mathrm{H}$ ZSM-5 ${ }^{[23]}$. In addition, the synthesis of ethyl benzene (the precursor of styrene and thus polystyrene) by alkylation of benzene with ethylene over H-ZSM-5 and the production 
of cumene (an intermediate to produce phenol and acetone) are other examples of largescale industries using zeolite catalysts ${ }^{[24]}$.

An excellent example of the use of zeolite catalysts in economically valuable applications that are important in daily life is the production of gasoline, olefins and other hydrocarbons from methanol which is obtained from various resources (Figure 1.2) ${ }^{[25]}$.

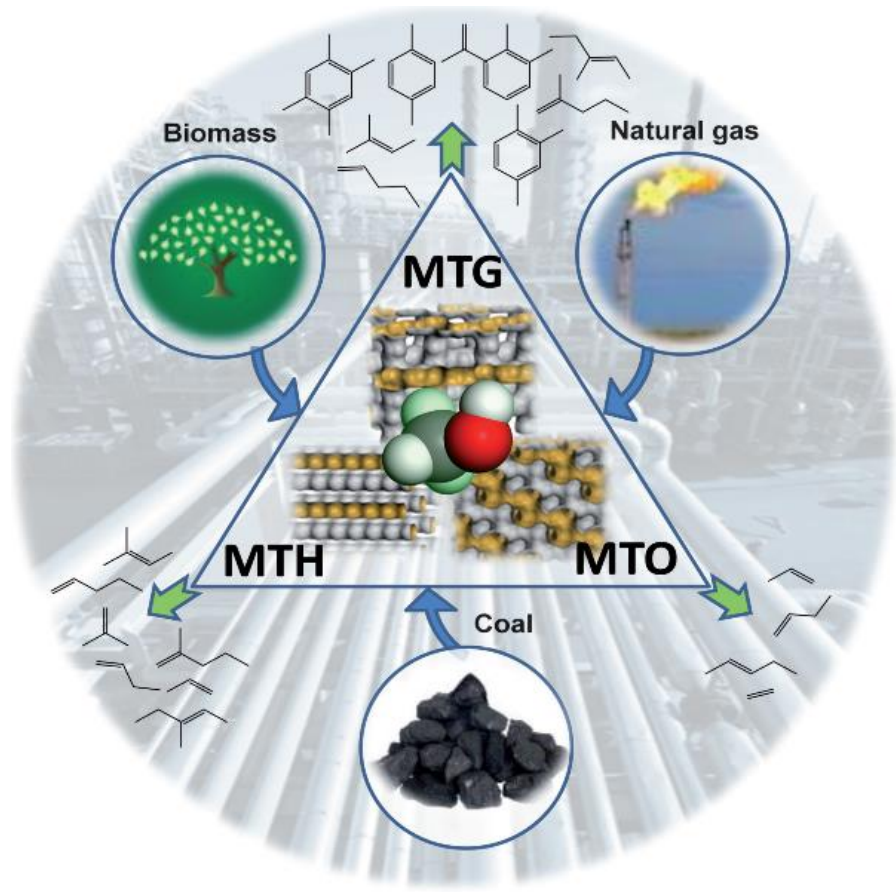

Figure 1.2 Conversion of methanol to olefins and other hydrocarbons over a zeolite catalyst (taken from ref. ${ }^{[25]}$ with permission).

Although zeolites are very efficient catalysts for many widespread applications, their use in the field of fine chemicals such as pharmaceuticals or even in the catalytic cracking of heavy hydrocarbons remains limited. This is because of their restricted micropore diameter $(<1 \mathrm{~nm})$ which is their main limitation ${ }^{[26]}$. This can be described as the confinement effect of the molecular sieves or mass-transfer constraints, in which small pores prevent larger molecules entering the pores and reducing the diffusion rate of smaller molecules being released out of the pores ${ }^{[26 a]}$. For example, microporous HZSM, H-Beta and H-Y zeolites exhibit poor catalytic reactivity for the benzylation of aromatics (Friedel-Crafts type alkylation reactions) ${ }^{[27]}$. Attempts to overcome this 
limitation in zeolites have resulted in the development of a great diversity of materials with controlled porosities and a variety of functionalities.

\subsubsection{Mesoporous silicates and aluminosilicates}

The discovery of a new family of mesoporous silicates (M41S) by Mobil Corporation researchers in 1992 with well-defined pores in the range of 2-50 $\mathrm{nm}$ has provoked much interest and raised expectations that these materials might be useful catalysts ${ }^{[28]}$. The most representative materials from this family are MCM-41 (with a hexagonal pore structure) and MCM-48 (cubic structure), as shown in Figure 1.3. These materials, with properties intermediate between amorphous silica-alumina and zeolites, have much larger pores compared with zeolites and therefore overcome the diffusional limitations associated with microporous zeolites.

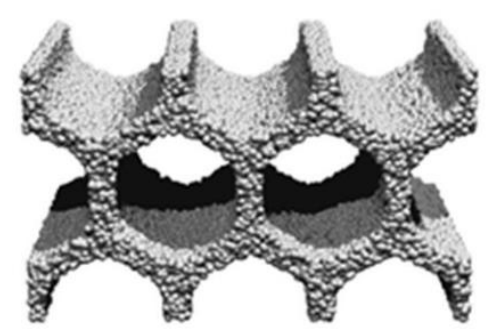

MCM-41

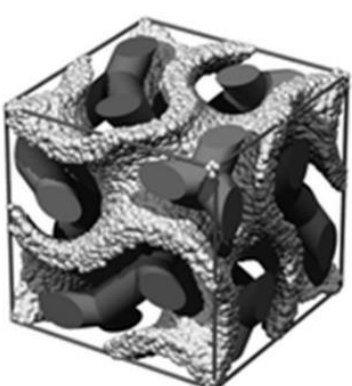

MCM-48

Figure 1.3 Structural representation of MCM-41 (without surfactant), and MCM-48 (with surfactant) ${ }^{[29]}$.

Despite their well-defined mesopores, these materials suffer from their low or medium acidity compared with zeolites, which is ascribed to a larger $(\mathrm{Si}-\mathrm{O}-\mathrm{M})$ bond angle

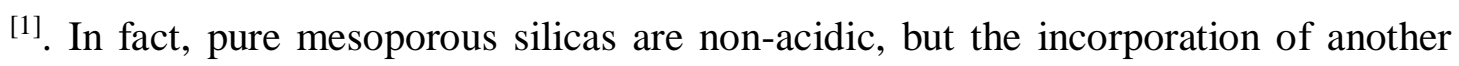
metals such as $\mathrm{Al}$ or metal oxides into the silica framework generates the active sites. Nonetheless, their main drawback is their low hydrothermal stability, which is ascribed to the amorphous nature of the internal walls, in fact on a microscale level these materials are actually amorphous ${ }^{[1,28]}$. For instance, MCM-41 has very good thermal stability in which a structure collapse could only occur at $\sim 1123 \mathrm{~K}$, but it has very low hydrothermal stability even in boiling water ${ }^{[30]}$. These limitations hinder their use in a number of applications such as catalysis or ion-exchange in aqueous solutions even at mild temperatures. 
What seemed to be more promising, however, are the mesoporous zeolites or the hierarchical zeolites, which contain both micro and mesopores. These materials are prepared by creating mesopores, $(2 \mathrm{~nm}<$ pore diameter (Dpore) $<50 \mathrm{~nm}$ ) inside the zeolite crystals which have the high acidity and thermal stability of the microporous zeolite while maintaining the advantage of being mesoporous (no mass transfer hindrance) ${ }^{[31]}$. These have been used in several applications where the microporous zeolites are inactive or show very poor activity, for instance, in the catalytic cracking of heavy hydrocarbons, alkylation of bulk aromatics and condensation reactions (see scheme 1.2) ${ }^{[32]}$.

Scheme 1.2 A comparison of the catalytic performance of micro- and mesoporous zeolite with MFI topology (redrawn from ref. ${ }^{[32]}$ )

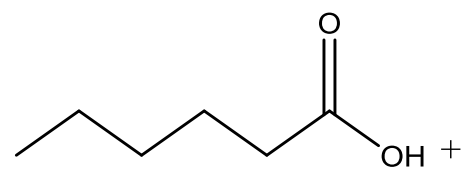

Conversion

MFI-meso $\quad 75 \%$

MFI $\quad 5 \%$

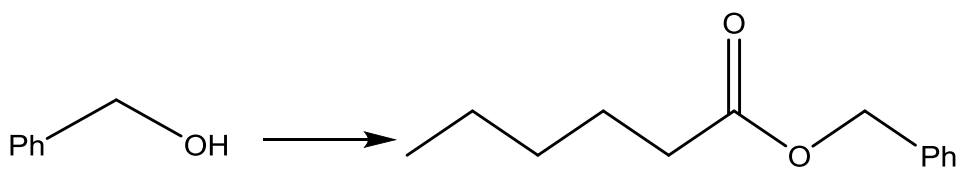

Selectivity

$79 \%$

$100 \%$

Two approaches are usually followed for the synthesis of mesoporous or hierarchical zeolites; these are the "top down" and "bottom up" approaches ${ }^{[33]}$. The former approach introduces secondary mesopores into the zeolite structure via post-synthetic treatments which include dealumination, removal of $\mathrm{Al}$ from the zeolite framework by thermal or acid treatments, and desilication, which is usually achieved by base treatments, e.g. with $\mathrm{NaOH}$. Although this approach is relatively inexpensive, it usually causes structural deformation leading to loss of crystallinity ${ }^{[31]}$.

In the "bottom up" approach, the zeolite is synthesised with the desired porosity by employing templating techniques such as soft or hard templating, similar in principle to the techniques that is used for synthesising mesoporous silicates (Figure 1.4).

In the hard-templating strategy, a secondary meso-scale template such as carbon nanotubes or mesoporous carbon is used to introduce the mesoporosity in the zeolites crystals. In the soft templating strategy, self-assembly surfactants are used which aggregate in aqueous solution to form micelles and then act as structural directing 
agents. In another type of soft templating, the mesoporosity is generated by an interaction between the silicates and the surfactant, forming inorganic-organic composites. These templating agents are removed from the mesoporous structure by calcination. Further details of these synthetic procedures have been well reviewed elsewhere ${ }^{[26]}$.

These sophisticated synthetic procedures require the use of expensive and sometimes toxic OSDAs which may militate against the large-scale production and commercialisation of hierarchical zeolites despite their excellent features ${ }^{[17,33 a]}$.

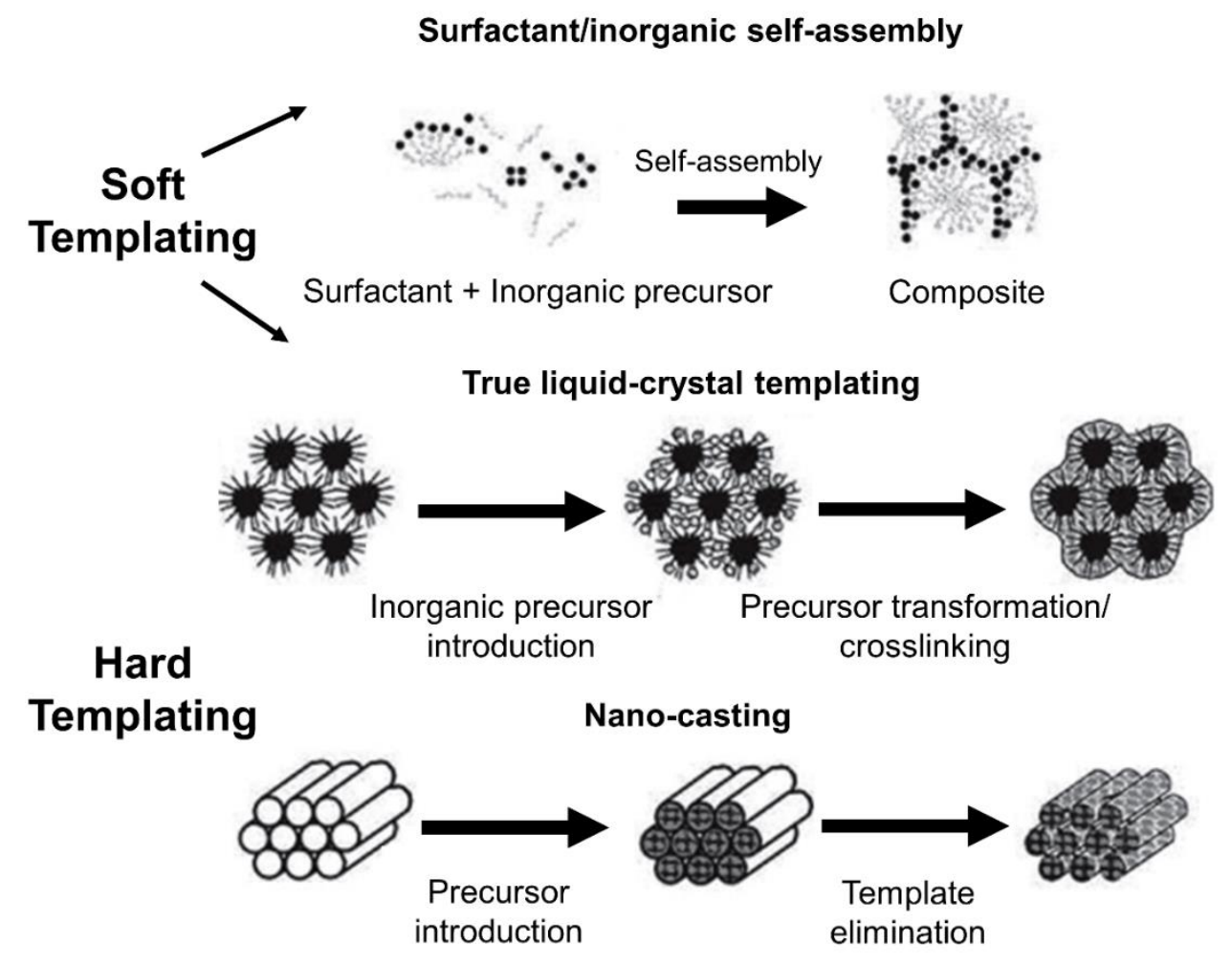

Figure 1.4 soft and hard-templating methods for mesoporous silicates synthesis (reproduced from ref. ${ }^{[34]}$ with permission).

\subsubsection{Clays and clay-supported catalysts}

Clays have played a significant role in heterogeneous catalysis since their original use as acids in the early 1930s, and have also been used as supports for stoichiometric reagents ${ }^{[35]}$. Subsequently they were replaced by amorphous silica-alumina and then by zeolites in the mid-1960s due to the negative impact of their impurities such as metals; furthermore, zeolites show better selectivity and stronger acidity ${ }^{[36]}$. However, 
the restricted pore size of zeolites, as mentioned above, has revived an interest in clays as catalysts.

Clays are crystalline layered silicate minerals, with structures composed of two types of layers as the main building blocks; these are an uncharged tetrahedral silica layer and a negatively charged octahedral layer formed very commonly by $\mathrm{Al}$. These layers are arranged either in an alternating manner (1:1) such as in kaolinite and halloysite, or in a sandwiched manner in which the octahedral layer is sandwiched between two tetrahedral layers $(2: 1)$ such as smectite ${ }^{[37]}$, as described in Figure 1.5. In the latter case, some of the tetrahedral silicon is substituted by $\mathrm{Al}$, generating negative charge that is compensated by the interlayer cations and can undergo ion-exchange.

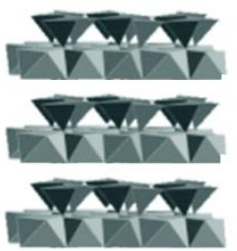

Kaolinite

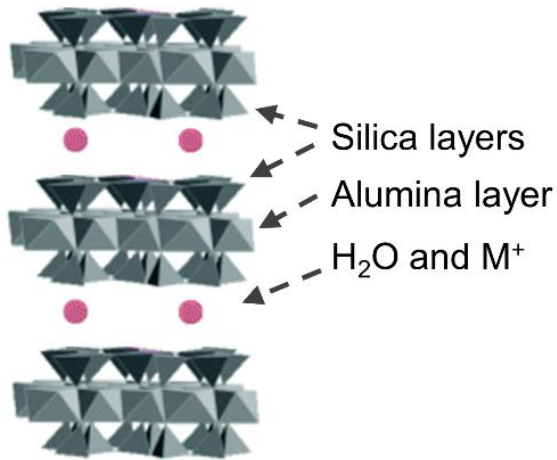

Smectite

Figure 1.5 Representation of kaolinite and Smectite clays structure, showing the arrangements of silica and alumina layers (reproduced from ref. ${ }^{[38]}$ ).

Clays have interesting properties such as the ability to act as ion-exchangers and catalyst supports, in addition to their ability for "pillaring" (Figure 1.6). Pillaring is a technique in which the two structural layers are held apart by using a pillaring agent such as inorganic polyoxocations $\left[\mathrm{Al}_{13} \mathrm{O}_{4}(\mathrm{OH})_{24} \mathrm{H}_{2} \mathrm{O}_{12}\right]^{7+}{ }^{[12]}$. Pillared clays have the advantage of greater thermal stability, since the presence of the pillaring agent prevents the layers from collapsing at elevated temperatures. 


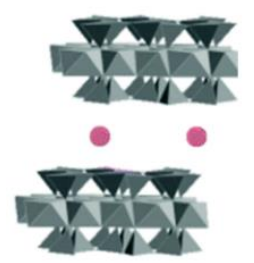

Organoammonium Cations

$\left(\mathrm{C}_{18} 2 \mathrm{C}_{1} \mathrm{~N}^{+}\right)$

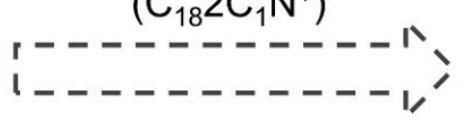

Smectite

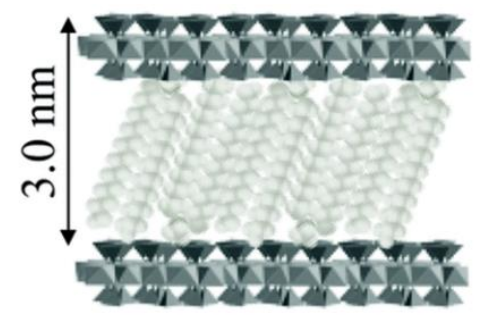

Figure 1.6 Illustration of clay pillaring (reproduced from ref. ${ }^{[38]}$ ).

Of the several clays such as halloysite and kaolinite that have been tested as catalysts for several catalytic processes, the commercially available montmorillonite (K10), an acid treated form of bentonite, has proved to be the most successful candidate ${ }^{[35]}$. Since the base clays show very poor catalytic activity, a post treatment such as thermal activation, acid treatment and ion-exchange, is necessary.

However, in order to improve their catalytic performance, most clays have been used mainly as supports for other catalytically active reagents such as $\mathrm{AlCl}_{3}$ and $\mathrm{ZnCl}_{2}$ or metals such as $\mathrm{Fe}$ or $\mathrm{Cu}{ }^{[35,37,39]}$. In the early 1980 s two active supported-clay catalysts were developed by impregnating Fe III and Cu II nitrates on K10 clay, commonly referred to as "clayfen" and "claycop" respectively ${ }^{[40]}$. These are efficient catalysts for the oxidation of alcohols to aldehydes, dihydropyridines to pyridines and regeneration of carbonyls from their protected forms such as phenylhydrazones ${ }^{[37]}$.

An important breakthrough in the development of clay-based catalysts was the discovery in 1989 by Clark and co-workers of a very active clay-based catalyst "clayzic" ${ }^{[41]}$ which uses $\mathrm{K} 10$ as a support for $\mathrm{ZnCl}_{2}$. This catalyst is very active, mainly in the Friedel-Crafts alkylation of benzene in which it is able to catalyse the benzylation of benzene with benzyl chloride with $100 \%$ conversion in $15 \mathrm{~min}$ at room temperature and over $80 \%$ selectivity for diphynelmethane (DPM) ${ }^{[35]}$.

This catalyst received much interest to explain its high activity, which is surprising since $\mathrm{ZnCl}_{2}$ is a relatively weak acid and $\mathrm{AlCl}_{3}$ is very active, although in the supported form with $\mathrm{K} 10$ the latter is inactive. One explanation for the higher reactivity of clayzic compared to its components is its ability to thermally dehydrate $\mathrm{ZnCl}_{2}$ in the presence of a support, forming Lewis acid sites, while similar treatment of $\mathrm{ZnCl}_{2}$ alone causes partial decomposition of the salt leading to poor reactivity ${ }^{[35]}$. 
Clay-based catalysts, whether in their acidic form, the acid post-treated clay, or as supports have been used to catalyse a wide range of reactions such as the oligomerisation of alkenes, addition reactions such as addition of alcohols to olefins to form ethers, Beckmann rearrangements, and Friedel-Crafts alkylation reactions ${ }^{[37,42]}$.

However, limitations of such catalysts arise from a weak interaction between the support and reagent, which is basically a physical attraction (physisorption). This results in leaching of the reagent in aqueous solutions ${ }^{[43]}$. In addition, some of these catalysts are surprisingly inactive in certain reactions, which limits their suitability for many applications. For instance, clayzic is not active in the alkylation of anisole, or in acylation reactions. It is also inactive to carboxylic acids (e.g. benzoic acids) and alkyl halides (except benzylic species) ${ }^{[43-44]}$. Drawbacks have also been reported in the use of clayfen in oxidation reactions; these include the need for excessive amounts of solvents, long reaction times, high ratios of reagent to substrate, and the generation of solid wastes ${ }^{[45]}$.

\subsubsection{Oxides and mixed oxides based catalysts}

In order to overcome the problems of catalyst instability and the limited reusability of the traditional physisorbed supported reagents, efforts have been made to develop true catalysts in which the reagent is chemically bound to the support. For this purpose, several materials were developed in the late 1990s, including organic-inorganic hybrid composites (e.g. Nafion-silica) and Lewis acids supported on modified mesoporous silica (e.g. $\mathrm{H}_{3} \mathrm{PO}_{4}-\mathrm{SiO}_{2}$ and $\left.\mathrm{AlCl}_{3}-\mathrm{SiO}_{2}\right){ }^{[43,46]}$.

The former materials possess interesting properties such as high acidity, high surface area, and large pore volume (20-60 nm), but although they appear promising for lowtemperature reactions such as aromatic alkylation, nitration and acylation and alcohol dehydration, their use is relatively uncommon, possibly due to the high synthesis cost of such catalysts ${ }^{[46-47]}$.

Acid treatment of surface hydroxylated mesoporous silica with $\mathrm{AlCl}_{3}$ reported by Wilson and Clark ${ }^{[48]}$ has led to acidity, interpreted by these authors in terms of the complexation of Lewis acid with the neighbouring oxygen as a base, to produce the highly acidic Bronsted site as shown in Figure 1.7. The catalytic activity of this catalyst, 
mainly for Friedel-Crafts benzylation of benzene, gave higher activity and selectivity over the homogeneous $\mathrm{AlCl}_{3}$ catalyst.

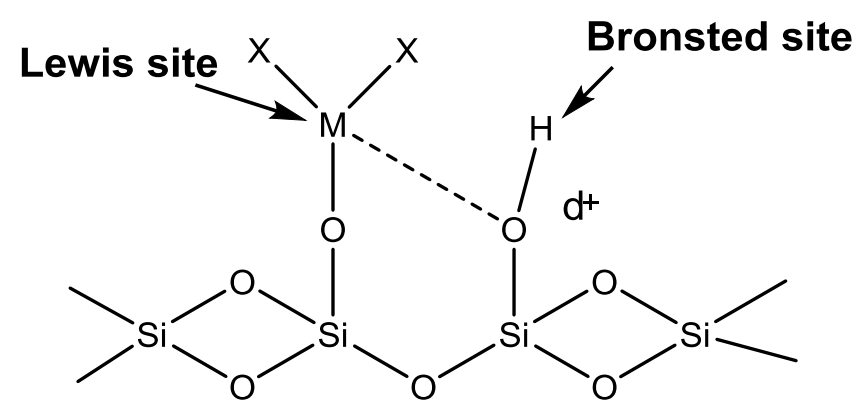

Figure 1.7 Illustration of Bronsted acidity induced by coordinated Lewis acid site ${ }^{[48]}$.

Another type of catalyst developed during the past decade is based on transition metal oxides such as $\mathrm{SnO}_{2}, \mathrm{TiO}_{2}$, and $\mathrm{ZrO}_{2}$. Although the acidity of such oxides is very low, it was found that functionalising their surfaces with sulfuric acid or ammonium sulfate (sulfated metal oxides) produces very high acidity (super acidity, $-13 \leq \mathrm{H}_{\mathrm{o}} \leq-16.3$ ) and therefore they show very high activity ${ }^{[49]}$. Among these oxides, mesoporous $\mathrm{ZrO}_{2}$ (zirconia) is the most popular and useful catalyst due to its excellent thermal and chemical stability.

The nature of the active sites is a matter of debate ${ }^{[36,49-50]}$; however, Figure 1.8 shows a widely accepted structure to describe the acidic sites of the sulfated zirconia (SZ). This model illustrates the formation of a sulfate bridge between two $\mathrm{Zr}$ atoms, and some of the Lewis acid sites are then transformed to Bronsted acid sites by absorption of water.

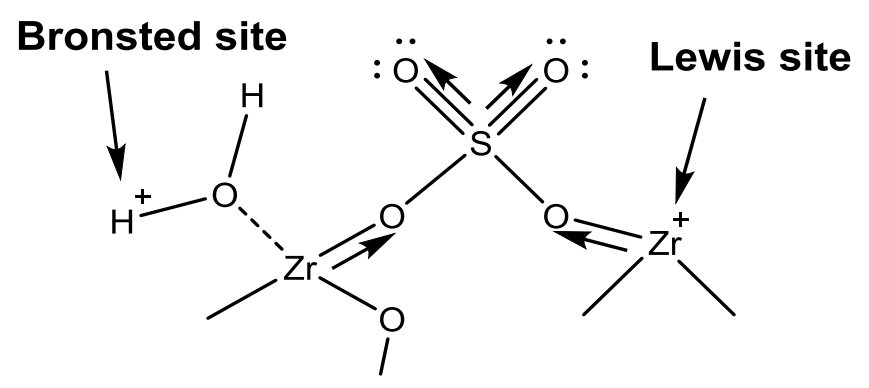

Figure 1.8 Illustration of the surface acidity of sulfated $\mathrm{ZrO}_{2}{ }^{[51]}$.

SZ shows very high activity for some organic synthesis applications e.g. Friedel-Crafts acylation of benzene, isomerisation of 1,2-epoxides and unsaturated hydrocarbons, and 
condensation of hydroquinone with aniline ${ }^{[49,52]}$. However, many studies have reported that these catalysts seem to suffer from leaching of the $S$ during calcination and regeneration which affects their activity and reusability ${ }^{[53]}$. For that reason, attempts to find an alternative to SZ have led to the development of tungstated zirconia (WZ), $\mathrm{WO}_{3} / \mathrm{ZrO}_{2}$. This catalyst gave the same activity as $\mathrm{SZ}$ but appears to be more stable and suitable for industrial applications ${ }^{[46,53 a]}$. The active sites in this catalyst are not well understood at present ${ }^{[53 \mathrm{~b}]}$, but a recent study claimed that the active centres consist of $\mathrm{WO}_{\mathrm{x}}$ clusters incorporating zirconium cations ${ }^{[54]}$.

\subsubsection{Heteropolyacids}

Heteropolyacids (HPAs) consist of heteropoly anions (oxoanions) with a central unit composed of typically $\mathrm{Si}$ or P. This central metal is tetrahedrally coordinated with oxygen and surrounded by 2-18 oxygen atoms connected hexagonally to metal atom, usually Mo or W. Figure 1.9 shows the structure of the phosphotungstate anion, the conjugate base of phosphotungstic acid $\left(\mathrm{H}_{3}\left[\mathrm{PW}_{12} \mathrm{O}_{40}\right]\right)^{[55]}$. Various acidic centres exist in HPAs, for instance, protonic acidic sites such as $\mathrm{H}_{3}\left[\mathrm{PW}_{12} \mathrm{O}_{40}\right]$ or Lewis acid sites such as $\mathrm{La}^{\mathrm{III}}\left[\mathrm{PMo}_{12} \mathrm{O}_{40}\right]$ in which a metal counter-ion acts as a Lewis acid ${ }^{[56]}$.

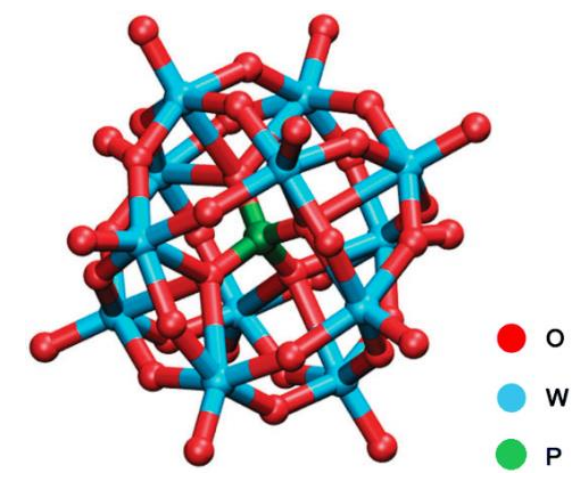

Figure 1.9 phosphotungstate anion ${ }^{[55]}$

Due to their low surface area $\left(5-8 \mathrm{~m}^{2} / \mathrm{g}\right)$, HPAs are most often used as supported catalysts rather than in the bulk form ${ }^{[57]}$. Various supports such as amorphous silica, zirconia and mesoporous silica MCM-41 have been employed for this purpose ${ }^{\text {[57-58]. }}$ The supported HPAs are of scientific interest and their use has been reported in several applications such as Friedel-crafts acylation and Fries rearrangement of aryl esters for which they show very high catalytic activity ${ }^{[56]}$. 
Nevertheless, their use is still limited, even in the supported form, due to some drawbacks. For instance, a high loading of HPAs may cause framework destruction of the support, or blockage of the pores of the support by the formation of clusters, thus negatively affecting their catalytic performance ${ }^{[58]}$. In addition, HPAs may leach from the support and catalyse the reaction homogeneously as a result of their high solubility in polar solvents ${ }^{[57]}$.

\subsubsection{Amorphous silica-alumina (ASA)}

ASAs are some of the earliest heterogeneous solid acids after the clays, having been used from the 1940s until recently in the petrochemical industries for hydrocracking, isomerisation and alkylation reactions ${ }^{[59]}$. They are usually prepared by mixing sources of $\mathrm{Si}$ and $\mathrm{Al}$ oxides (commonly commercially available silica or sodium silicate with aluminium nitrate or ammonium aluminium hexafluoride salt), followed by filtration of the solid, drying at $\sim 400 \mathrm{~K}$ and finally calcination at a high temperature $(750-1000 \mathrm{~K})$ ${ }^{[60]}$. If one or both of the sources contains sodium, ion-exchange with $\mathrm{NH}_{4}{ }^{+}$is necessary in order to remove the alkali cations.

ASA consists of various weight percentages of $\mathrm{Al}$ in three different forms (tetrahedral, octahedral and $\mathrm{Al}_{2} \mathrm{O}_{3}$ ) grafted onto a silica backbone which is usually tetrahedral ${ }^{[60]}$. The ratios of the aluminium in the various coordination states depend on the preparation method as well as the weight \% of Al. For example, when ASA is prepared by grafting $\mathrm{Al}$ into silica gel most of the $\mathrm{Al}$ is in the form of $\mathrm{Al}_{2} \mathrm{O}_{3}$, whereas preparation by cogelation produces $\mathrm{Al}$ as $\mathrm{Al}^{\mathrm{IV}}$ and $\mathrm{Al}^{\mathrm{VI}}{ }^{[60 \mathrm{~b}]}$. It has been found that ASA with a higher $\mathrm{Al}$ content contains a higher proportion of octahedral $\mathrm{Al}$ which is believed to result from the formation of $\mathrm{Al}_{2} \mathrm{O}_{3}$ clusters ${ }^{[61]}$. The small fraction of tetrahedral $\mathrm{Al}$ is formed due to the substitution of Si by Al during the preparation or subsequent calcination, and these species form Bronsted acidic sites which are the bridging hydroxyl group ( $\mathrm{Si}$ $\mathrm{OH}-\mathrm{Al}^{\mathrm{IV}}$ ), as described earlier ${ }^{[60]}$. The strength of these sites increases with increasing $\mathrm{Al}^{\mathrm{IV}} / \mathrm{Al}^{\mathrm{VI}}$ ratio. These two coordinated $\mathrm{Al}$ sites are also responsible for generating Lewis acidic sites ${ }^{[60 \mathrm{~b}]}$. This may explain the weaker activity of ASA compared with zeolites, in which, in some cases, only a few $\mathrm{Al}$ atoms substitute for $\mathrm{Si}$, resulting in weak acidity.

Similar to clay based catalysts, ASAs have been replaced by synthetic zeolites which have higher activities and longer lifetimes than ASA. The larger pore volumes of the 
ASA sometimes result in higher selectivity compared to zeolites, by allowing more molecules to enter the active sites ${ }^{[62]}$. However, the large pores allow increased coke deposition in the active sites, causing deactivation of the catalyst. It is not only the large pore volume which causes greater coke formation, but it has also been claimed that the presence of $\mathrm{Al}_{2} \mathrm{O}_{3}$ in the ASA is responsible for coke formation ${ }^{[63]}$. In addition, the complexity of the ASA's surface compared to zeolites prevents the ability to tailor the acidity of the ASA, providing another reason why such catalysts became less desirable after the introduction of synthetic zeolites.

In summary, this chapter describes the most common solid acid catalysts in terms of their preparation techniques, their structural properties, and the nature of their active sites. Their catalytic activities in some important industrial applications have also been discussed, together with some of the limitations associated with these catalysts. This chapter also outlines some of the wide range of applications where homogeneous catalysts have been replaced by more environmentally friendly heterogeneous catalysts.

Nonetheless, $\sim 95 \%$ of heterogeneous catalytic processes are in the bulk petrochemical industries with only very limited applications in the fine chemical industries ( $\sim 3-5 \%$ of fine chemicals are produced via heterogeneous catalysts) ${ }^{[64]}$. This is ascribed to the limitations of the solid catalysts such as the zeolites, where their applications are hindered by their restricted micropores. The low hydrothermal stability of mesoporous M-silicates (such as MCM-41) causes structural deformation at high temperature in aqueous solution. Other highly promising solid catalysts suffer drawbacks of high cost, sophisticated synthesis procedures, and environmental risks from the use of toxic reagents.

Thus, there remains a need for the development of new efficient and environmentally benign solid catalysts that can be prepared readily with tailored chemical and physical properties.

\subsection{Scope of this thesis}

This thesis describes the synthesis and characterisation of amorphous aluminosilicate inorganic polymers (also known as geopolymers) as a novel class of solid acid heterogeneous catalysts for liquid-phase organic synthesis applications. 
The similar chemical and thermal stability of geopolymers to their crystalline analogues (zeolites) ${ }^{[65]}$ suggests the possibility that they may be suitable candidates for heterogeneous catalysis. Moreover, geopolymers can readily be prepared at ambient temperature from environmentally friendly natural raw materials, or from industrial wastes such as fly ash. Unlike zeolites or mesoporous silicates, micro, meso or even hierarchical porosity can be introduced into the geopolymer structure by varying the synthesis composition without the use of costly, and sometimes toxic, OSDAs ${ }^{[66] . ~ I n ~ t h i s ~ r e s e a r c h, ~}$ acidic active sites will be generated in the geopolymer framework by ionexchange with ammonium ions followed by thermal treatment, allowing the nature of these acidic sites (Bronsted or Lewis) to be tailored to specific catalytic applications [67]. These possibilities suggest that geopolymers may have considerable potential as cost-effective, efficient, readily synthesised and environmentally friendly heterogeneous solid acid catalysts.

\subsubsection{Geopolymers}

Geopolymers are X-ray amorphous aluminosilicate polymers, first described by Joseph Davidovits in late 1970s ${ }^{[68]}$. Their structure consists of randomly organized threedimensional arrangements of tetrahedral silicate and aluminate units joined through their common oxygen atoms ${ }^{[69]}$. Charge balance in the tetrahedral aluminate units is achieved by the presence of (usually) monovalent alkali ions, Figure 1.10 illustrates a schematic representation of the geopolymer framework.

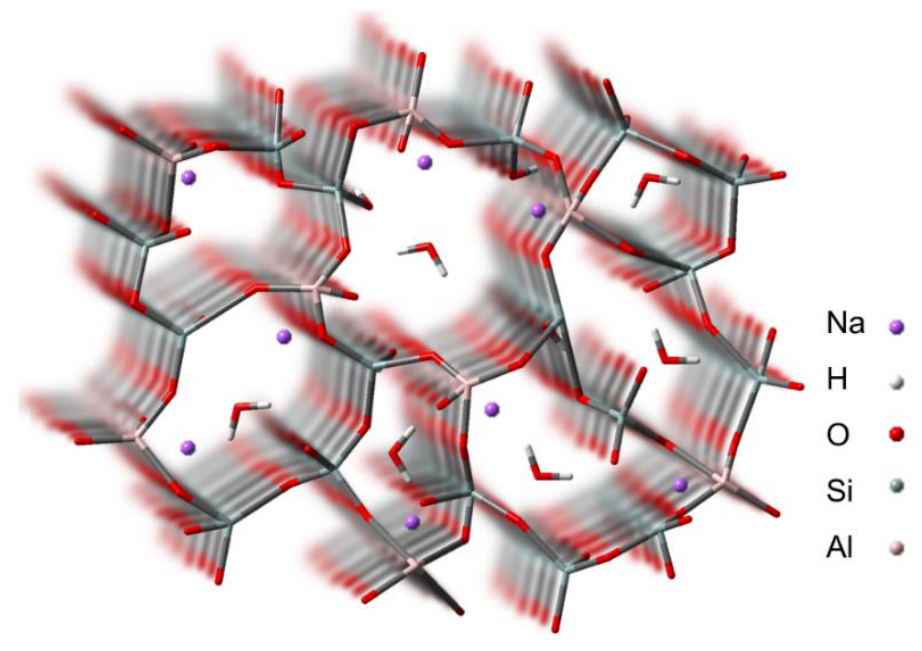

Figure 1.10 Schematic representation of the geopolymer framework 
Davidovits and his team have described geopolymers as polysialates (sialate is an abbreviation for silicon-oxo-aluminate) ${ }^{[70]}$. They have also identified three different building blocks based on the alternating silica and alumina tetrahedral units that form the geopolymer framework (scheme 1.3).

Scheme 1.3 Three different main building units of a geopolymer

Polysialate

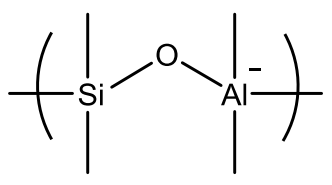

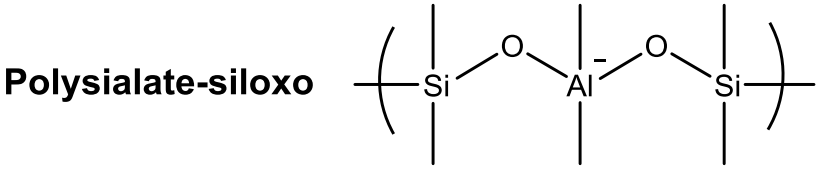<smiles>CC(C)(O[GeH3])[SiH2]O[Si](C)(C)O[Si](C)(C)O[Si](C)(C)O[Si](C)(C)C</smiles>

Geopolymers are usually prepared by mixing a solid aluminosilicate such dehydroxylated kaolin clay with an alkali metal silicate under alkaline conditions, the mixture setting to a hard mass at ambient temperatures. The process starts with dissolution of the aluminosilicate clay in the alkaline solution, and therefore the crystalline clay must typically be thermally activated to break down the crystalline structure, causing the coordination of Al to change from 6 to a mixture of 4, 5, and 6 which facilitates the dissolution process ${ }^{[69]}$. The resulting deconstructed $\mathrm{Si}$ and $\mathrm{Al}$ species then polymerise into a gel composed of monomers. As the concentration of these monomers increases, they grow to form the geopolymer framework ${ }^{[71]}$.

The setting properties of aluminosilicate geopolymers are dependent on their composition, particularly their $\mathrm{SiO}_{2} / \mathrm{Al}_{2} \mathrm{O}_{3}$ molar ratio, which is normally stated to have an optimum value of about 3, although geopolymers with higher and lower silica contents have been shown to be viable ${ }^{[72]}$.

Due to their excellent mechanical properties, geopolymers have conventionally been applied as environmentally friendly alternatives to Portland cement ${ }^{[73]}$. In previous work, we have developed natural fibre-reinforced geopolymer-based 
composites, using wool and flax fibres, for low-technology applications such as construction or insulation materials ${ }^{[74]}$. Due to their porous three dimensional framework, geopolymers have been used as adsorbents in waste water treatment applications and for the storage and immobilization of heavy metals and radioactive wastes ${ }^{[75]}$.

More recently, geopolymers have been investigated for more advanced applications such as drug delivery media ${ }^{[76]}$, and we have demonstrated their use as a novel stationary phase for open-column chromatography where silica or alumina would commonly be used ${ }^{[77]}$. The chromatographic performance of the geopolymer was comparable with or superior to the conventional stationary phases silica and alumina, with greater chemical stability to polar solvents than silica and lower preparation costs. However, geopolymers have only very rarely been used as supports in heterogeneous catalysis rather than catalysts in their own right.

\subsubsection{Geopolymers in heterogeneous catalysis}

Despite their desirable properties, only very few recent studies have reported the possibility of using geopolymers in the field of heterogeneous catalysis. Sazama et al. have reported the use of geopolymers as redox catalysts for the reduction of NOx by ammonia and the oxidation of volatile hydrocarbons where the active catalyst was $\mathrm{Pt}$, $\mathrm{Fe}, \mathrm{Cu}$ or $\mathrm{Co}$, supported on a geopolymer matrix ${ }^{[78]}$. The performance of the geopolymer catalysts was superior to conventional vanadium oxides supported on alumina $\left(\mathrm{V}_{2} \mathrm{O}_{5} / \mathrm{Al}_{2} \mathrm{O}_{3}\right)$. Geopolymers have also been used to support other catalytic nanoparticles such as $\mathrm{TiO}_{2}{ }^{[79]}, \mathrm{CuO}^{[80]}$, and a combination of both ${ }^{[81]}$ for photocatalytic degradation of organic pollutants. Sharma and co-workers have also reported the use of geopolymers loaded with $\mathrm{Ca}^{2+}$ as base catalysts for the generation of biofuel ${ }^{[82]}$. Of these studies, only Sharma et. al. has reported the reusability of their prepared catalysts, showing that deactivation began to occur after the third reaction cycle. As mentioned above, the main drawback of supported catalysts is the tendency of their active species to leach from the host in aqueous solutions, causing deactivation and affecting the catalyst reusability. 


\subsubsection{Novelty and research approaches}

The aim of this research is to synthesise and characterise geopolymers with properties suitable as novel solid acid catalysts, in which the active sites are generated within their structure rather than simply using the geopolymer as a support for other catalytically active species. The formation of these acidic centres as part of the geopolymer framework, and the investigation of their catalytic properties for several industrially important organic reactions has not previously been reported. The catalytic properties of these new materials were shown to be markedly improved by applying post-synthetic treatments to tailor their acidity and porosity, making them suitable for a number of organic reactions, each of which requires different active sites.

This project followed three different approaches. Firstly, clay-based geopolymer-based catalysts were synthesised with high surface areas and weak surface acidities, H-bonded silanol nests and vicinal silanols, these are the properties required to catalyse an important class of organic reactions, the Beckmann rearrangement reaction. In this section of the work, the new geopolymer catalysts were tested in a model Beckmann reaction, the rearrangement of cyclohexanone oxime to caprolactam. This part of the work is described in detail in chapter 3.

Secondly, it was shown that mesoporous and hierarchical clay-based geopolymer catalysts could be synthesised which contain both Bronsted and Lewis acid sites such as required for the catalysis of another class of important organic reactions, the FriedelCrafts benzylation reactions. The performance of the new catalysts with acid sites tailored for this purpose was demonstrated in a systematic study of the Friedel-Crafts benzylation of several relatively large aromatics. This part of the work is presented in chapter 4 .

Thirdly, several geopolymer catalysts were prepared from fly ash, a waste material from the combustion of coal. This contains $\mathrm{Fe}_{2} \mathrm{O}_{3}$, which could potentially provide active sites in addition to those synthesised within the geopolymer structure itself. The synthesis and characterization of these catalysts is reported and their performance in Friedel-Crafts benzylation and Friedel-Crafts acylation reactions were demonstrated. This work forms chapters 5 and 6 of the thesis. 


\section{Chapter 2}

\section{Experimental}

This chapter describes the synthesis of the parent geopolymers and the subsequent preparation of the catalysts. It provides a full description of the characterisation procedures and a brief description of the techniques commonly used to characterise solid catalysts. The last part of the chapter (section 2.3) describes the analysis of the catalytic reactions in which the reaction outcomes were identified and quantified. In addition, it discusses some common models that provide valid and accurate measurements of the heterogeneous catalytic reaction kinetics by evaluating the heat and mass transfer effects.

\subsection{Synthesis and catalysts preparation}

\subsubsection{Geopolymer synthesis (the section was rearranged)}

The geopolymers in this study were prepared from two different sources; clay and fly ash. The clay was a New Zealand kaolinite-type halloysite clay (Imerys Premium Grade). Four different fly ashes (FA) were collected from different sources; a C-class (high $\mathrm{CaO}$ content) fly ash was collected from the Huntly power station, New Zealand. The three other fly ashes were F-class (low $\mathrm{CaO}$ content), and were from Australian power stations; Gladstone in Queensland, Hyrock from Bayswater station, and Mount Piper station both the latter located in New South Wales. Table 2.1 summerises the composition of the halloysite clay and the four fly ashes. 
Table 2.1 Chemical composition of the halloysite clay and the fly ashes ${ }^{\text {a }}$

\begin{tabular}{cccccc}
\hline Oxide (wt. \%) & Halloysite & Hyrock & Huntly & Gladstone (G.S.) & Mount piper \\
\hline $\mathrm{SiO}_{2}$ & 49.5 & 66.68 & 46.48 & 43.61 & 69.23 \\
$\mathrm{Al}_{2} \mathrm{O}_{3}$ & 35.5 & 22.46 & 19.1 & 31.28 & 23.89 \\
$\mathrm{Na}_{2} \mathrm{O}$ & 0.04 & 0.18 & 0.76 & 0.46 & 0.22 \\
$\mathrm{~K} 2 \mathrm{O}$ & 0.01 & 1.05 & 0.69 & 0.31 & 2.23 \\
$\mathrm{MgO}$ & 0.02 & 0.56 & 2.85 & 1.62 & 0.24 \\
$\mathrm{CaO}$ & 0.02 & 1.09 & 15.56 & 3.73 & 0.23 \\
$\mathrm{Fe}_{2} \mathrm{O}_{3}$ & 0.29 & 5.79 & 10.2 & 12.74 & 1.15 \\
$\mathrm{TiO}_{2}$ & 0.09 & 0.99 & 1.19 & 1.76 & 0.92 \\
$\mathrm{MnO}$ & 0.00 & 0.09 & 0.08 & 0.17 & 0.02 \\
$\mathrm{P}_{2} \mathrm{O}_{5}$ & 0.00 & 0.18 & 0.27 & 1.10 & 0.11 \\
$\mathrm{SO}_{3}$ & 0.00 & $<0.01$ & 0.45 & $<0.01$ & $<0.01$ \\
$\mathrm{~L}_{0} \mathrm{O} . \mathrm{I}$. & 13.8 & 0.72 & 1.04 & 2.51 & 1.07 \\
$\mathrm{SUM}$ & 99.27 & 99.78 & 98.67 & 99.3 & 99.29 \\
\hline
\end{tabular}

${ }^{a}$ determined by X-ray fluorescence analysis (XRF).

\subsubsection{Clay based geopolymer}

Geopolymers with two different alkali cations $\left(\mathrm{Na}^{+}\right.$and $\left.\mathrm{K}^{+}\right)$were synthesised in this project. The masses of all the components used in the synthesis of all the prepared geopolymers are shown in Table 2.2. The weighed amounts of analytical grade $\mathrm{NaOH}$ or KOH (Panreac) were dissolved in distilled water and then sodium silicate (Sod-SilD) (FERNZ Chemical Co, NZ, Type “D”, $\mathrm{Na}_{2} \mathrm{O} / \mathrm{SiO}_{2}=0.48$, solids content $=41.1$ mass $\%$ ) or potassium silicate (Type K66, Ineos Silicas, UK) was added to the mixture. The solution was cooled to room temperature in an ice bath. The dehydroxylated clay, prepared by heating at $600{ }^{\circ} \mathrm{C}$ for $12 \mathrm{hr}$ was then gradually added to the mixture. After thorough manual mixing for $10 \mathrm{~min}$. the geopolymer resins were cured in covered plastic molds at $80{ }^{\circ} \mathrm{C}$ for $6 \mathrm{hr}$., then uncovered and oven-dried at $40{ }^{\circ} \mathrm{C}$ overnight. The hardened blocks were then broken into pieces and ground in a vibratory mill (Bleuler, Switzerland) fitted with a tungsten carbide pot and milling rings and sieved to pass a $105 \mu \mathrm{m}$ mesh. 
Table 2.2 weights in grams (g) of all the components used in the geopolymers synthesis

\begin{tabular}{cccccccccc}
\hline Geopolymer & NaOH & KOH & $\mathbf{H}_{2} \mathbf{O}$ & Na-silicate & K-silicate & Halloysite & Corresponding-FA & Silica-fume & Alphabond \\
\hline Na-N & 16.1 & 0.0 & 32.3 & 64.2 & 0.0 & 80.0 & 0.0 & 0.0 & 0.0 \\
Na-hiSi & 40.0 & 0.0 & 80.1 & 80.0 & 0.0 & 80.0 & 0.0 & 60.0 & 0.0 \\
K-N & 0.0 & 9.0 & 18.0 & 0.0 & 32.0 & 40.0 & 0.0 & 0.0 & 0.0 \\
K-hiSi & 0.0 & 11.0 & 20.0 & 0.0 & 20.0 & 30.0 & 0.0 & 15.0 & 0.0 \\
Geo-Hyrock & 7.0 & 0.0 & 17.0 & 0.0 & 0.0 & 0.0 & 21.0 & 0.0 & 2.0 \\
Geo-Huntly & 4.0 & 0.0 & 8.0 & 15.0 & 0.0 & 0.0 & 30.0 & 0.0 & 2.5 \\
Geo-G.S. & 4.0 & 0.0 & 8.0 & 15.0 & 0.0 & 0.0 & 20.0 & 0.0 & 0.0 \\
Geo-Mt. piper & 4.0 & 0.0 & 8.0 & 15.0 & 0.0 & 0.0 & 30.0 & 0.0 & 4.0 \\
\hline
\end{tabular}


Two sodium and two potassium-based geopolymers were prepared with "normal" compositions (e.g. $\mathrm{SiO}_{2} / \mathrm{Al}_{2} \mathrm{O}_{3} \sim 3.5$ ), designated Na-N and $\mathrm{K}-\mathrm{N}$, and two silica-rich compositions, containing twice this amount of silica, designated Na-hiSi and K-hiSi were also prepared. The compositions of the high-silica geopolymers were adjusted by the addition of fine silica fume (Elkem 971-U, Elkem, Norway) simultaneously with the clay (see Table 2.2 for masses). The composition of all the clay based geopolymers is shown in Table 2.3.

Table 2.3 Chemical composition of the synthesised clay based geopolymers ${ }^{\text {a }}$

\begin{tabular}{ccccc}
\hline Molar ratio & Na-N & Na-hiSi & K-N & K-hiSi \\
\hline $\mathrm{SiO}_{2} / \mathrm{Al}_{2} \mathrm{O}_{3}$ & 3.54 & 6.40 & 3.59 & 5.19 \\
$\mathrm{Na}_{2} \mathrm{O} / \mathrm{Al}_{2} \mathrm{O}_{3}$ & 1.26 & 2.27 & 0.02 & 0.02 \\
$\mathrm{~K}_{2} \mathrm{O} / \mathrm{Al}_{2} \mathrm{O}_{3}$ & 0.01 & 0.00 & 1.11 & 1.30 \\
$\mathrm{H}_{2} \mathrm{O} / \mathrm{Al}_{2} \mathrm{O}_{3}$ & 13.59 & 22.88 & 13.26 & 14.81 \\
$\mathrm{ZrO}_{2} / \mathrm{Al}_{2} \mathrm{O}_{3}$ & 0.00 & 0.07 & 0.00 & 0.05 \\
$\mathrm{CaO} \mathrm{Al}_{2} \mathrm{O}_{3}$ & 0.00 & 0.00 & 0.00 & 0.00 \\
$\mathrm{Fe}_{2} \mathrm{O}_{3} / \mathrm{Al}_{2} \mathrm{O}_{3}$ & 0.00 & 0.00 & 0.00 & 0.00 \\
\hline
\end{tabular}

${ }^{\text {a }}$ determined by XRF.

\subsubsection{Fly ash based geopolymer}

Unlike hydrated crystalline clay minerals, fly ash is a product of a thermal process (burning coal) and it is composed of a heterogeneous mixture of metal oxides, making thermal pre-treatment unnecessary. Thus, all the fly ash samples were used as received with no further chemical or thermal treatments. Only one geopolymer composition (the "normal" composition, $\mathrm{SiO}_{2} / \mathrm{Al}_{2} \mathrm{O}_{3} \sim 3.5$ ) was prepared, and only in the $\mathrm{Na}$ form. Accordingly, the four fly ash based geopolymers were designated with the name of the fly ash, plus the abbreviation "Geo" (e.g. Geo-Hyrock). The chemical compositions of all the synthesized fly ash geopolymers are shown in Table 2.4. The fly ash-based geopolymers were synthesised by exactly the same procedure as used for the clay-based geopolymers (see section 2.1.1.1 and Table 2.2), but in some of the compositions the $\mathrm{SiO}_{2} / \mathrm{Al}_{2} \mathrm{O}_{3}$ ratio had to be adjusted by the addition of amorphous $\mathrm{Al}_{2} \mathrm{O}_{3}$ (Alphabond 300, Alcoa). 
Table 2.4 Molar compositions of the synthesised fly ash-based geopolymers ${ }^{\text {a }}$

\begin{tabular}{lcccc}
\hline Molar ratio & Geo-Hyrock & Geo-Huntly & Geo-G.S. & Geo-Mt. piper \\
\hline $\mathrm{SiO}_{2} / \mathrm{Al}_{2} \mathrm{O}_{3}$ & 3.55 & 3.79 & 3.56 & 3.84 \\
$\mathrm{Na}_{2} \mathrm{O} / \mathrm{Al}_{2} \mathrm{O}_{3}$ & 1.34 & 1.10 & 1.41 & 0.79 \\
$\mathrm{~K}_{2} \mathrm{O} / \mathrm{Al}_{2} \mathrm{O}_{3}$ & 0.04 & 0.03 & 0.01 & 0.00 \\
$\mathrm{H}_{2} \mathrm{O} / \mathrm{Al}_{2} \mathrm{O}_{3}$ & 14.32 & 11.23 & 14.81 & 8.31 \\
$\mathrm{ZrO}_{2} / \mathrm{Al}_{2} \mathrm{O}_{3}$ & 0.00 & 0.00 & 0.00 & 0.00 \\
$\mathrm{CaO} \mathrm{Al}_{2} \mathrm{O}_{3}$ & 0.06 & 1.04 & 0.22 & 0.01 \\
$\mathrm{Fe}_{2} \mathrm{O}_{3} / \mathrm{Al}_{2} \mathrm{O}_{3}$ & 0.12 & 0.24 & 0.26 & 0.02 \\
$\mathrm{TiO}_{2} / \mathrm{Al}_{2} \mathrm{O}_{3}$ & 0.04 & 0.60 & 0.07 & 0.03 \\
\hline
\end{tabular}

${ }^{a}$ determined by XRF.

\subsubsection{Catalyst preparation}

The acidic form of each geopolymer was obtained by converting it to the $\mathrm{NH}_{4}{ }^{+}$form by ion exchange, followed by thermal treatment to decompose the ammonium and produce the H-form of the geopolymer. Throughout the thesis unless otherwise stated, the heating, for both $\mathrm{NH}_{4}{ }^{+}$decomposition and clay dehydroxylation, was performed in a regular lab furnace under static air atmosphere with a starting heating rate of $15^{\circ} \mathrm{C} / \mathrm{min}$.

Ion-exchange of the alkali for $\mathrm{NH}_{4}{ }^{+}$was carried out by the method of O'Connor et al. ${ }^{[83]}$. One gram of geopolymer powder was treated with $100 \mathrm{ml}$ of $0.1 \mathrm{M} \mathrm{NH}_{4} \mathrm{Cl}$ solution (Panreac) with vigorous stirring at room temperature for $12 \mathrm{hr}$., washed thoroughly with a freshly prepared solution of $0.1 \mathrm{M} \mathrm{NH}_{4} \mathrm{Cl}$, filtered, then washed again with distilled water to remove any remaining alkali ions and dried at $40{ }^{\circ} \mathrm{C}$ overnight. Zeolites $\mathrm{H}-$ ZSM-5 and Y were used for comparison purposes in the catalytic reactions and were ion-exchanged in the same manner.

The ion-exchanged catalysts were then heated at a range of temperatures between 300 $-550{ }^{\circ} \mathrm{C}$ to determine the appropriate temperature to produce acidic sites necessary to catalyze the particular organic reaction being studied; accordingly, the $\mathrm{NH}_{4}{ }^{+}$-ion exchanged geopolymers were heated to $450{ }^{\circ} \mathrm{C}$ for use as the catalyst in the Beckmann 
rearrangement, while catalysts that were used in the Friedel-Crafts alkylation and acylation reactions were heated to $550{ }^{\circ} \mathrm{C}$. The heating time was generally 15 min unless otherwise stated; a range of heating times up to $5 \mathrm{hr}$. was investigated but longer heating times were shown unnecessary for some the tested reactions. This is an advantage over zeolites which require several hours of thermal activation. Both zeolites used in this study were thermally treated in exactly the same way as the geopolymers.

\subsubsection{Post synthetic treatment}

Dealumination is routinely employed with zeolitic materials to introduce secondary mesopores or remove the EFAl ${ }^{[84]}$. This is commonly achieved by applying strong acids such as $\mathrm{HCL}$ or $\mathrm{HNO}_{3}$ or chelating agents such as $\mathrm{Na}_{2} \mathrm{H}_{2}$ EDTA. The latter has been shown to selectively remove the EFAl rather than the FAl and thus having minor influence on the zeolitic crystallinity ${ }^{[85]}$. More recently, desilication by base treatment (usually $\mathrm{NaOH}$ ) has been widely applied to zeolites (mainly with high $\mathrm{Si} / \mathrm{Al}$ ratio) as it introduces connected intracrystaline mesoporosity that cannot be achieved by dealumination ${ }^{[86]}$. However, this basic post-synthetic treatment was found ${ }^{[33 a]}$ not effective when applied to zeolites with low Si/Al ratios (e.g. Y zeolite), and therefore a pre-dealumination step is necessary to introduce mesoporosity in the framework of these high-Al zeolites. Furthermore, Verboekend et. al. have reported that an additional step of mild acid treatment after the alkaline treatment (desilication) further improves the porosity by removing any remaining Al-rich debris that could block the pores ${ }^{[33 a]}$. In this study these sequential treatments (see below) were applied to the geopolymers to improve their porosity and acidity and thus their catalytic activity.

It should be noted that post synthetic treatments were only applied to the clay based geopolymers. These were sequentially dealuminated and desilicated after ion exchange, following the procedure of Verboekend et al. ${ }^{[33 a]}$ in which a weighed amount of the $\mathrm{NH}_{4}{ }^{+}$-geopolymer catalyst was first dealuminated by treatment with $20 \mathrm{ml} / \mathrm{g} 0.11 \mathrm{M}$ $\mathrm{Na}_{2} \mathrm{H}_{2}$ EDTA (Merck) for $5 \mathrm{hr}$. at $85^{\circ} \mathrm{C}$; these samples were designated DA (e.g. NahiSi-DA). This was followed by desilication by treatment with $30 \mathrm{ml} / \mathrm{g} 0.1 \mathrm{M} \mathrm{NaOH}$ for $30 \mathrm{~min}$ at $65{ }^{\circ} \mathrm{C}$. The desilication was carried out in a plastic container placed in a thermostatic bath. The third step of the treatment was washing with $0.11 \mathrm{M} \mathrm{Na}_{2} \mathrm{H}_{2}$ EDTA, performed as in the first dealumination but for only $2 \mathrm{hr}$. Between each of these 
steps, the solid was filtered, washed with distilled water and dried at $\sim 50{ }^{\circ} \mathrm{C}$ overnight. Finally, these samples were exchanged with $\mathrm{NH}_{4}{ }^{+}$as described above, but in three 6hr. treatments with $\mathrm{NH}_{4} \mathrm{Cl}$ solution. The geopolymer samples treated in this way are designated Seq (e.g. Na-hiSi-Seq).

As discussed above, the initial step of the treatment (dealumination) was carried out with the $\mathrm{NH}_{4}{ }^{+}$-form of geopolymer. This is based on the reported experimental finding that the disodium form of EDTA ( $\left.\mathrm{Na}_{2} \mathrm{H}_{2} \mathrm{EDTA}\right)$ has no significant effect in terms of dealumination on the sodium form of zeolites with low $\mathrm{Si} / \mathrm{Al}$ ratio (e.g. Na-Y zeolite) [33a, 87]. Therefore, when $\mathrm{Na}_{2} \mathrm{H}_{2}$ EDTA is used, it is commonly applied to the ionexchanged form of the zeolite, whereas the acid form $\mathrm{H}_{4}$ EDTA is usually applied to either form (the alkali cation and the ammonium ion-exchanged zeolite). Thus, in this study, $\mathrm{Na}_{2} \mathrm{H}_{2}$ EDTA was used in conjunction with the $\mathrm{NH}_{4}{ }^{+}$-geopolymer. It should be noted that the catalysts preparation, including $\mathrm{NH}_{4}{ }^{+}$ion-exchange and the sequential treatment, was repeated several times and no significant change in their reproducibility in terms of catalytic performance was observed.

\subsection{Catalyst characterisation}

\subsubsection{X-ray diffraction (XRD)}

$\mathrm{XRD}$ is a very useful tool, widely applied to the characterization of solid materials, typically used to identify the structure of solid crystalline phases. A diffracted X-ray from a crystal results in a distinctive diffraction pattern that can be used as a fingerprint to identify that particular crystal structure.

On the other hand, amorphous materials such as geopolymers do not possess a crystalline structure with long range order and therefore XRD does not provide useful information about their structure. In practice, X-ray interaction with an amorphous material produces a broad background feature of the scattered X-rays. However, even for amorphous materials such as geopolymers, XRD is a very useful technique since it demonstrates the formation of the amorphous material. In addition, it reveals the presence of any crystalline phases that may be present either as deliberately introduced phases (e.g. nanoparticles) loaded on the solid or present as impurities. Moreover, in applications where amorphous materials are subjected to thermal treatments, XRD is 
useful in measuring their thermal stability by indicating whether or not a recrystallization process has occurred. Below is a brief description of the principle of the XRD.

Atoms within a crystal are arranged in a repeating array with long range order forming a three-dimensional lattice. Since the X-ray wavelength is of the order of the intracrystalline spacing, it is diffracted by the crystal, providing information about its structure. The arrangement of the atoms in the lattice can be described in terms of planes of atoms, defined by three integers (hkl), called Miller indices. When an X-ray beam interacts with the surface of a crystalline solid, a unique diffraction pattern is obtained in a form of peaks. Each of these peaks is related to a diffracted X-ray from a specific plane.

The condition for a diffraction to occur is illustrated in figure 2.1. A constructive interference is obtained when Bragg's law is satisfied ${ }^{[88]}$. As the wavelength of the Xray $(\lambda)$ is fixed by the nature of the target material in the X-ray tube, a diffracted X-ray from particular parallel planes of atoms with spacing (d) will be in phase only at a specific angle. The incident angle $(\boldsymbol{\theta})$ is the angle between the X-ray source and the sample, the diffracted angle ( $2 \boldsymbol{\theta})$ is defined as the angle between the incident angle and the detector, and thus the detector is set at an angle of $2 \boldsymbol{\theta}$.

\section{Bragg's law: $\mathrm{n} \lambda=2 \mathrm{~d} \sin \theta$}

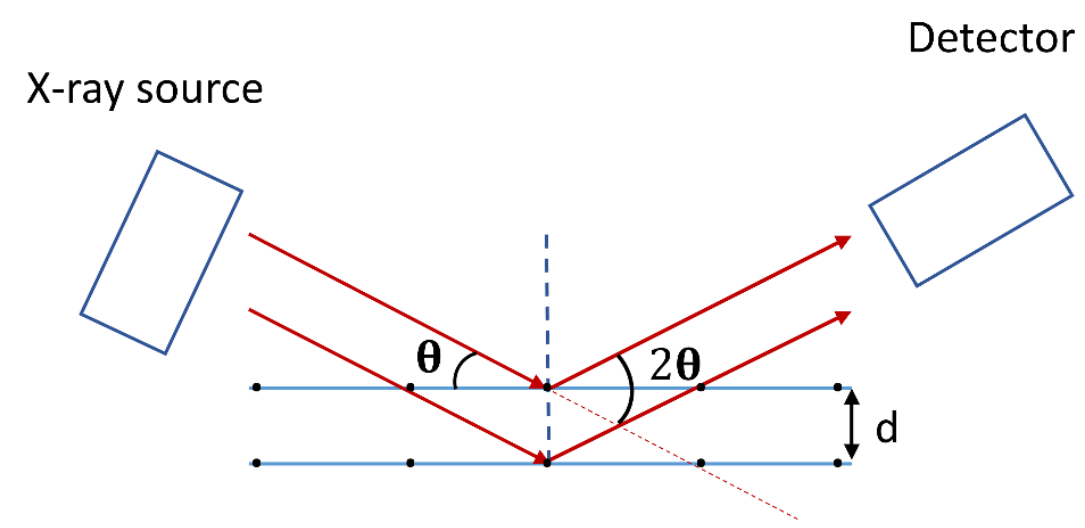

Figure 2.1 Illustration of XRD theory.

The X-ray source is basically a vacuum tube containing a metal filament (usually W) and a cathode target, commonly $\mathrm{Cu}$ or Mo. When the filament is connected to a high 
voltage it generates high energy electron beam $(30-50 \mathrm{kV})$ which is accelerated towards the anode by the potential difference. When the beam strikes the cathode, it causes expulsion of an electron from the inner shell ( $\mathrm{K}$ shell). When an electron from a higher shell (the M or L shells) fills the electron hole, the energy difference is released mostly in a form of heat and a characteristic X-ray whose energy and thus wavelength is specific for each individual atom. The resulting X-ray beam is passed through a collimator, producing a monochromatic X-ray beam.

Where powder samples are exposed to the X-ray beam, in all the possible diffraction reflections are observed, as there will always be crystals in the beam that satisfy Bragg's law for a specific set of atomic planes.

In this work, X-ray powder diffraction was used to characterize the formation of geopolymer as well as the structural stability of the thermally treated geopolymers and the spent geopolymers catalysts. A Bruker D8 Avance X-ray diffractometer with Nifiltered $\mathrm{Cu} \mathrm{K \alpha}$ radiation operated at $45 \mathrm{kV}$ and $40 \mathrm{~mA}$ was used, and the XRD data were analyzed by comparing the diffraction peak patterns with those collected in the International Center for Diffraction Data (ICDD) database.

\subsubsection{X-ray fluorescence (XRF)}

The compositions of the raw materials from which the geopolymers were synthesised as well as all the synthesized geopolymers were obtained by XRF analysis. The principle of generating X-rays is as described in section (2.2.1). The sample is presented to the X-rays which cause ionization of the inner shells of the different atoms present. The resulting characteristic emitted X-rays are detected, allowing qualitative and quantitative analysis of the sample's composition. This technique is applied only for atoms heavier than $\mathrm{Ne}$ due to the low X-ray yields for lighter elements ${ }^{[89]}$. The present XRF analyses were carried out by a commercial analytical company, SpectraChem Services, Seaview.

\subsection{3 ${ }^{27} \mathrm{Al}$ and ${ }^{29} \mathrm{Si}$ MAS NMR}

Magic angle spinning nuclear magnetic resonance (MAS NMR) experiments are routinely applied in characterisation of solid catalysts, providing key structural data that cannot be obtained by XRD. In aluminosilicates such as zeolites or geopolymers ${ }^{27} \mathrm{Al}$ and ${ }^{29} \mathrm{Si}$ MAS 
NMR experiments are essential to determine the coordination environment of the $\mathrm{Al}$ and the $\mathrm{Si}$, and thus giving insight to the arrangement of the aluminate and silicate units within the framework. Moreover, it is useful in evaluating the impact of post-synthetic treatments on the structure of the solid catalyst, by showing the transformation of a metal from one coordination state to another, or leaching of an element from the framework. ${ }^{27} \mathrm{Al},{ }^{1} \mathrm{H}$ and ${ }^{17}$ O MAS NMR spectra are also very useful tools to study the acidity of solid acid catalysts

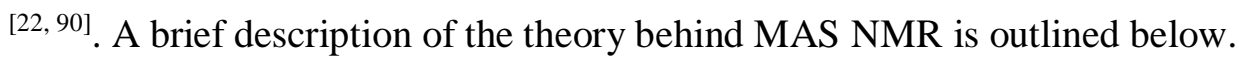

When a nucleus with a spin, (containing an odd number of protons and/or neutrons) is placed in a magnetic field, its spin energy states are split (Zeeman interaction). Transition between these states requires the absorption or emission of a photon in the radiofrequency (rf) range. In the NMR experiment the aim is to detect this rf resonance, which is unique for each nucleus in a different bonding environment.

When the nucleus is placed in an external magnetic field $\left(\mathrm{B}_{\mathrm{o}}\right)$, its spin magnetic moment precesses around the axis of $B_{o}$ at a specific frequency called the Larmor frequency (Lf) that is a characteristic of each nucleus. At this stage, the spin energy levels are split due to $\mathrm{B}_{\mathrm{o}}$ by the Zeeman effect and the nucleus magnetic moment is aligned in the lower energy level (at the same direction as the external $\mathrm{B}_{\mathrm{o}}$ ). Pulsing the nucleus with a rf pulse at the Lf causes the magnetic moment to incline from the axis of $\mathrm{B}_{\mathrm{o}}$ (the nucleus absorbs the rf and is transferred to higher spin energy levels with its spin magnetic moment aligned against $B_{o}$ ). When the pulsing ceases, the spin magnetic moment returns to its original axis (at the lower spin energy level) with a characteristic relaxation time, thereby inducing a voltage that is recorded as a function of time called a free induction decay (FID). The FID is transformed from the time domain to the frequency domain using a mathematical process called Fourier Transform (FT) as described in figure 2.2.

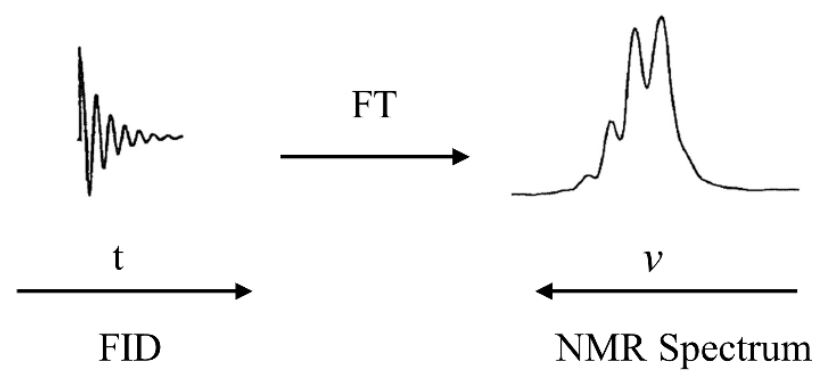

Figure 2.2 A representation of the Fourier transformation of FID to NMR spectrum ${ }^{[91]}$. 
Usually a nucleus is not only affected by the external $B_{o}$ but also by magnetic effects from neighbouring nuclei and shielding from neighbouring electrons; therefore it absorbs or emits slightly different frequencies from a single nucleus. Thus, it is difficult to measure the absolute NMR frequency, so the resonance frequency is measured relative to an external standard.

The complexity of solid state NMR compared to the liquid analogy is due to the broadening of the resulting peaks in the solid state NMR spectrum. This is due to a number of interactions around the nuclei, between dipole moments, between quadrupole moments (in quadrupole nuclei) and the electric field gradients at the nucleus, and also to anisotropy shielding effects ${ }^{[91]}$. In the liquid phase the rapid movement of the atoms cancels the impact of these interactions, resulting in narrow peaks, but in solids the atoms are not as free to move, giving rise to broadening by the various mechanisms listed above.

Some of these interactions in the solid sample are related to the angle between the sample and the magnetic field, expressed in terms of Legendre polynomials such as $\left(3 \cos ^{2} \boldsymbol{\theta}-1\right)$. This term becomes zero at the "magic angle" of $54.7^{\circ}$ to the axis of the magnetic field (figure 2.3a).
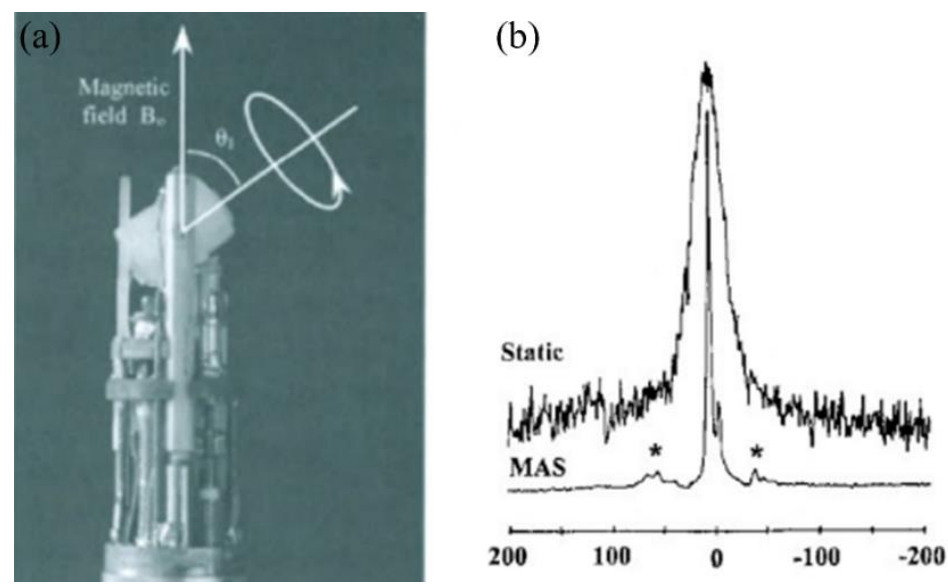

Figure 2.3 (a) magic angle spinning probe and (b) ${ }^{27} \mathrm{Al}$ NMR of a mixer of $\mathrm{Al}_{2} \mathrm{O}_{3}$ and $\mathrm{Y}_{2} \mathrm{O}$ measured for unspun (upper spectrum) and spun at the magic angle (bottom spectrum) ${ }^{[91]}$. The peaks marked with asterisks are spinning side bands.

Spinning the sample at high speed (up to 15000 revolutions/s) at the magic angle cancels broadening associated with non-quadrupolar $(I=1 / 2)$ and second order 
quadrupolar nuclei, resulting in narrow peaks (figure 2.3b); therefore, this technique is referred to as magic angle spinning, or MAS NMR.

Broadening that cannot be cancelled by MAS such as fourth order quadrupolar broadening can be removed by applying other techniques such as double angle spinning NMR or multi quantum MAS NMR, described in detail elsewhere ${ }^{[91-92]}$, but these techniques were not used in this work.

${ }^{27} \mathrm{Al}$ and ${ }^{29} \mathrm{Si}$ solid state MAS NMR spectra were acquired at a magnetic field of $11.7 \mathrm{~T}$ using a Bruker Avance I II 500 spectrometer operating at a ${ }^{27} \mathrm{Al}$ frequency of 130.24 $\mathrm{MHz}$ and a ${ }^{29} \mathrm{Si}$ frequency of $99.29 \mathrm{MHz}$. The $11.7 \mathrm{~T}{ }^{27} \mathrm{Al}$ solid-state spectra were acquired using a $4 \mathrm{~mm}$ Doty MAS probe with a silicon nitride rotor spun at $10-12 \mathrm{kHz}$, a $1 \mu$ s pulse and a $1 \mathrm{~s}$ recycle time, the spectra referenced with respect to $\mathrm{Al}\left(\mathrm{H}_{2} \mathrm{O}\right)^{3+}$. The ${ }^{29} \mathrm{Si}$ spectra were acquired with a $5 \mathrm{~mm}$ Doty MAS probe and a zirconia rotor spun at $\sim 6 \mathrm{kHz}$. The excitation pulse for ${ }^{29} \mathrm{Si}$ was $7 \mu \mathrm{s}$ with a recycle time of $30 \mathrm{~s}$ and the spectra were referenced with respect to tetramethylsilane (TMS).

\subsubsection{Electron microscopy}

Electron microscopy is another useful tool and widely applied in several fields such as medicine, biology and materials science. In the field of heterogeneous catalysis, it provides useful information about the morphology and porosity of the solid catalyst particles. In addition, it provides qualitative and quantitative elemental analysis of the catalyst.

The principle of electron microscopy is that electrons are used instead of light to produce images. The wavelength of an accelerated electron beam is $10^{5}$ times shorter than light wavelength ${ }^{[93]}$, therefore providing images of much higher resolution, down to few angstroms. Electromagnetic lenses are used in electron microscopes instead of glass lenses as in regular optical microscopes. The electron microscope techniques used in this work are described below.

\subsubsection{Scanning electron microscopy (SEM)}

In scanning electron microscopes, the sample surface is exposed to a high energy electron beam, accelerated by a potential difference, called the primary electron beam 
$(10-50 \mathrm{kV})$ which interacts with the sample as shown in figure 2.4. The secondary electrons are collected by an appropriate detector and processed to provide an image of the sample. Elemental analyses of the sample can also be obtained by detection and analysis of the emitted X-rays. Since the SEM data are obtained by collecting the scattered electrons from the sample surface, the experiment is conducted under high vacuum to avoid collisions between the electrons and the air.

A scanning electron microscope consists of an electron gun that generates the primary beam, commonly a heated tungsten filament. Since electrons interact with electric and magnetic fields, electromagnetic lenses (condenser and objective lenses) are used to focus the electric beam on to the sample. The primary electron beam is usually scanned over the sample in two perpendicular directions by a scanning coil, to cover a rectangular area of the sample called the raster. Different images are produced by collecting the secondary or backscattered electrons produced from each point of the raster. Thus, the SEM image is generated point by point, unlike the optical microscope which provides simultaneous imaging ${ }^{[94]}$.

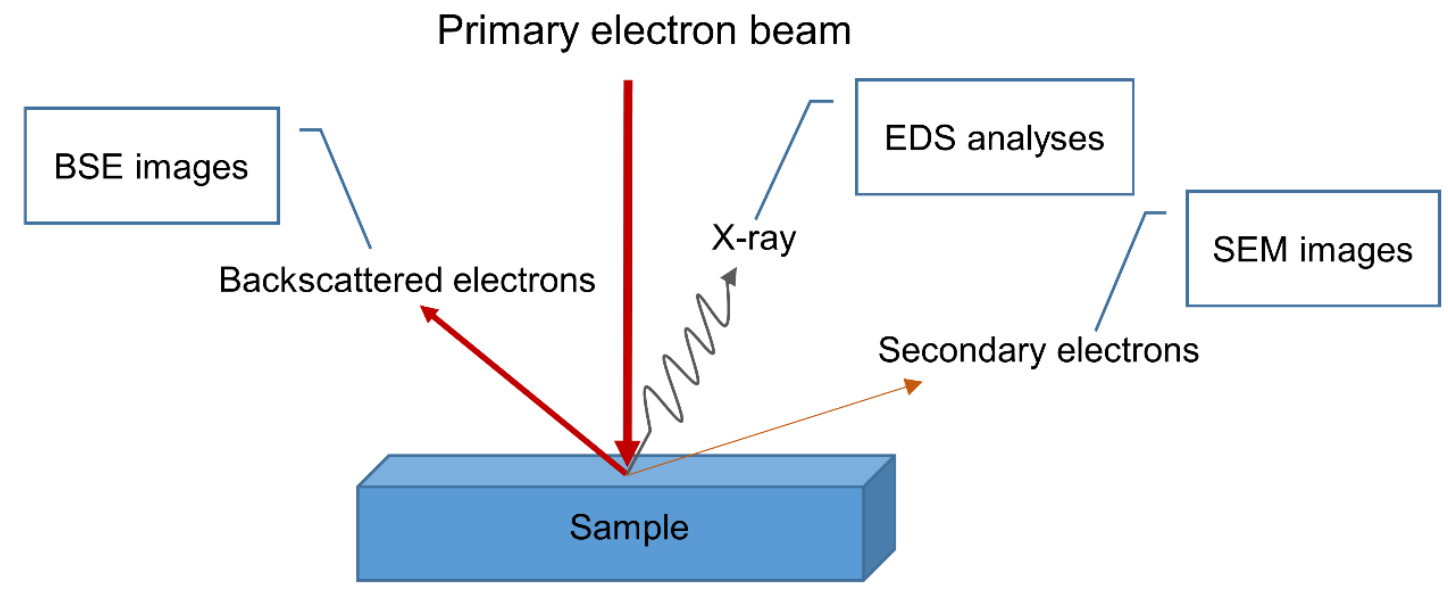

Figure 2.4 Illustration of some interactions between the primary electron beam and the sample in a scanning electron microscope (improved version from ${ }^{[95]}$ ).

SEM images are produced by the secondary electrons (figure 2.4), providing details about the surface topography and an estimate of the particle size. Up to $\sim 30,000 \mathrm{x}$ magnification with image resolution of $\sim 50 \mathrm{~nm}$ is achievable by regular SEM microscopes, but some SEMs using a field emission cathode in the electron gun produce a narrower beam of both low and high energy electrons, resulting in improved spatial resolution up to about $1.5 \mathrm{~nm}^{[89]}$. 
Backscattered electron images (BSE) are formed by the backscattered electrons, allowing discrimination of multiphase materials by variations in the contrast which depend on the atomic weight of the elements in the sample, e.g. the heavier atoms appear brighter.

\subsubsection{Energy dispersive X-ray fluorescence spectroscopy (EDS)}

As shown in figure 2.4, striking a sample with high energy beam of electrons produces X-rays with energy characteristic of each particular element in the sample (sections (2.2.1) and (2.2.2)). Measurement of these x-rays provides qualitative analyses of the elements in the sample. EDS can be used to map the distribution of the various elements in the sample.

\subsubsection{SEM sample preparation and apparatus}

The sample must be electrically conductive to avoid surface charging which results in poor imaging. Non-conductive samples are usually coated with an ultrathin layer of conductive material (few nanometres thick) such as carbon, Pt or Au. The choice of the conductive layer becomes important if elemental analysis (e.g. EDS and mapping) is required.

In this work, the sample powder was placed on a carbon tape and coated with a $\sim 16 \mathrm{~nm}$ layer of carbon using a Quorum Q150T turbo-pumped carbon coater. SEM images were obtained by using a JEOL JSM-6610 LA analytical scanning electron microscope operated at 10-20 kV, connected to an energy- dispersive spectrometer (EDS).

\subsubsection{Transmission electron microscopy (TEM)}

Transmission electron microscopes (TEMs) provide direct imaging of the target sample using electron beams of much higher energy $(200-300 \mathrm{kV})$ than in SEMs, thereby providing much higher magnification $\left(\times 10^{3}-10^{6}\right)$ with a spatial resolution of $0.2 \mathrm{~nm}$

${ }^{[93]}$. In the TEM, the high energy electron beam, focussed by a series of electromagnetic lenses, impinges on the sample surface and the transmitted electrons provide an image of the sample on a fluorescent screen placed under the sample. The image contains density variations depending on the thickness of various regions of the sample. TEM samples must be very thin (a few nanometres thick), and therefore this technique cannot be used for bulk specimens, for which SEM is more appropriate ${ }^{[93]}$. 
In the case of heterogeneous solid catalysts, TEM provides valuable information about their porosity, particularly their pore shapes that cannot be obtained by other techniques. Furthermore, the dispersion of nanoparticles supported on solid catalysts can be seen by TEM.

\subsubsection{Scanning transmission electron microscopy (STEM)}

STEM combines the techniques of TEM and SEM by scanning a very narrow spot on the sample and collecting the resulting X-rays, secondary, transmitted and backscattered electrons. Thus, STEM produces qualitative and quantitative elemental analyses similar to EDS element mapping. Usually TEMs are equipped with scanning coils to permit both TEM and STEM measurements.

\subsubsection{TEM sample preparation and apparatus}

In this work a slurry of the sample powder was produced by grinding in ethanol using a mortar and pestle. The slurry then was dispersed on copper grids, Formvar/carbon coated, followed by exposure of the samples to a low-energy plasma to clean the grids using a JEOL EC-2000 IC ion cleaner. TEM and STEM were acquired using a JEOL JSM-2100 F transmission electron microscope operated at $200 \mathrm{kV}$.

\subsubsection{Fourier transform infrared spectroscopy (FTIR)}

Vibrational spectroscopy, particularly FTIR, is another routine technique for characterising inorganic solid catalysts and solid and liquid organic compounds. In the case of solid catalysts, FTIR provides structural information by identifying the chemical bonds within the material. Moreover, ion-exchange processes and the subsequent generation of acidic hydroxyl groups are usually monitored by FTIR, which is also used to identify compounds adsorbed on the surface of the solid catalysts, thereby providing information regarding poisoning of spent catalysts or surface acidity by use of probe molecules (see section 2.2.6.2).

In this work, all the FTIR spectra were obtained by suspending the sample powder in a $\mathrm{KBr}$ disk and the spectra were acquired using a Perkin Elmer Spectrum One FTIR spectrometer in the range $4000-450 \mathrm{~cm}^{-1}$. The resulting spectra were interpreted by comparison with spectra in the literature. 


\subsubsection{Surface acidity characterisation}

Several techniques have been developed to qualitatively and quantitatively characterise the surface acidity of solid acid catalysts ${ }^{[96]}$. Some techniques provide a direct analysis of the Bronsted and/or Lewis acidic sites; these include solid-state ${ }^{1} \mathrm{H},{ }^{17} \mathrm{O}$ and ${ }^{27} \mathrm{Al}$ MAS NMR spectroscopy and FTIR spectroscopy in the characteristic region of hydroxyl groups $\left(3700-3400 \mathrm{~cm}^{-1}\right)$. These techniques can also be used to indirectly characterise surface acidity of a solid by use of probe molecules such as amines or carbon oxides. When a probe molecule is used, thermal desorption experiments can also be used to provide information about the amount of the desorbed species to provide an estimate of the concentration of the acidic sites. In addition, the strength of the acidic sites can be estimated by measuring the temperature at which the desorption occurs. Below is a brief description of the most common and viable techniques used for surface acidity characterisation.

\subsubsection{Acidity characterisation by solid state NMR spectroscopy}

Solid state NMR has been used in various ways to characterise the acidity of solid acids. ${ }^{1} \mathrm{H}$ MAS NMR has been used to distinguish between Bronsted acidic sites (bridging $\mathrm{OH}$ groups with chemical shifts at $\sim 4.0 \mathrm{ppm}$ ) and other silanol groups in zeolites ${ }^{[15}$, 97]. ${ }^{17} \mathrm{O}$ MAS NMR has also been used for the same purpose, but the very low natural abundance of ${ }^{17} \mathrm{O}(0.037 \%)$ and its large quadrupolar moment have limited its application ${ }^{[90 a, 97]}$.

${ }^{27} \mathrm{Al}$ MAS NMR spectroscopy is used to characterise framework $\mathrm{Al}$ (FAl) and extra framework $\mathrm{Al}$ (EFAl) which act as Lewis acidic sites in zeolites and zeolite-like materials. EFAl is usually in the form of penta-coordinated and octahedral Al, with characteristic chemical shifts at $30.5 \mathrm{ppm}$ and $0 \mathrm{ppm}$ respectively [21, 60b]. More recently, Lewis acidity, which arises from highly distorted tetrahedral and tricoordinated $\mathrm{FAl}$, has been detected as broad resonance at $\sim 40 \mathrm{ppm}$ and $\sim 67 \mathrm{ppm}$ respectively, using advanced solid-state NMR techniques such as ${ }^{27} \mathrm{Al}$ and ${ }^{1} \mathrm{H}$ REDOR (rotational-echo double-resonance triple-quantum) and CP (cross polarization triple-quantum) MAS NMR ${ }^{[22] .}$ 
When a probe molecule is used, such as an amine, ${ }^{15} \mathrm{~N}$ solid-state MAS NMR can be used to study interactions between the probe molecule and the acidic sites in the solid catalyst ${ }^{[98]}$.

\subsubsection{Acidity characterisation by FTIR spectroscopy}

FTIR in the range of $\left(3700-3400 \mathrm{~cm}^{-1}\right)$ provides direct information about acidic hydroxyl groups, whether weakly or non-acidic silanols or the bridging hydroxyls (Bronsted acid sites). Figure 2.5 illustrates the several $\mathrm{OH}$ groups that can be generated on the surface of silicates or aluminosilicates, together with their characteristic IR absorption ${ }^{[60 \mathrm{~b}, 99]}$.

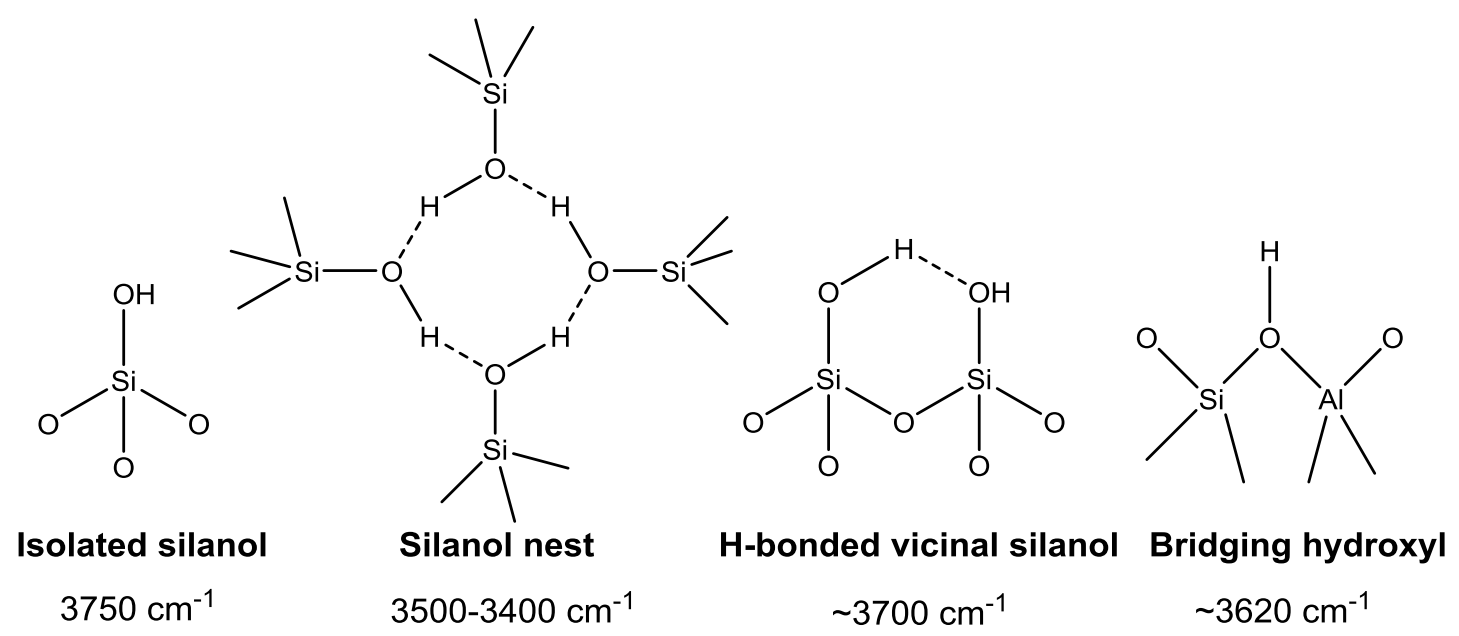

Figure 2.5 Illustration of hydroxyl groups in different environments in silicates and aluminosilicates and their distinctive IR absorption.

FTIR is the tool of choice for characterising the surface acidity using a basic probe molecule. Several molecular probes have been used for this purpose, the most common being pyridine ${ }^{[98]}$. Pyridine can distinguish between the acidic sites on the surface, since it interacts differently with Bronsted and Lewis acid sites, resulting in distinctive bands for each acidic site. Pyridine coordinated to Lewis acidic sites shows bands at 1448 and $1600 \mathrm{~cm}^{-1}$, while the bands at 1544 and $1638 \mathrm{~cm}^{-1}$ are characteristic of protonated pyridine on a Bronsted acid site ${ }^{[100]}$. Furthermore, quantitative analyses of the concentrations of the acidic sites can be made by integrating the absorption peaks [101]. 
In these experiments, the catalyst must be activated (degassed) prior to its exposure to pyridine, by heating at temperatures between $300-500{ }^{\circ} \mathrm{C}$ under vacuum (up to $10^{-6}$ Torr) for sufficient time to remove the moisture and any other physisorbed species on the catalyst surface ${ }^{[60 \mathrm{~b}, 100 \mathrm{a}]}$. Since it is important to measure the chemisorbed pyridine, after exposure of the catalyst to pyridine, the physisorbed pyridine is removed by heating under vacuum. The temperature and vacuum applied at this stage are determined by the acid strength of the catalyst, for instance the pyridine that is adsorbed on strong acid sites requires higher desorption temperature compared to that adsorbed on weaker acid sites.

These experiments are usually performed in situ to obtain more accurate results. For this purpose, special IR cells have been developed which are connected to temperaturecontrolled heaters and have windows for evacuation and supply of the pyridine ${ }^{[100 a]}$. In this equipment, the catalyst is placed in the cell as a self-supported wafer, and its activation under vacuum is monitored by measuring a background spectrum. The solid is then exposed to the pyridine for a period of time, followed by heating under vacuum to remove the physisorbed molecules, and the spectrum recorded. The two spectra are subtracted to give the final result.

\subsubsection{Thermal desorption of a molecular probe}

Thermal decomposition of a chemisorbed probe molecule does not only provide quantitative analysis of the amount of acidic sites, but also gives insights into the strength of the acidic sites, since the temperature of desorption is proportional to the strength of the acid site. A common technique to achieve this is temperature programmed desorption (TPD) of $\mathrm{NH}_{3}{ }^{[98]}$. Another convenient method is thermal gravimetric analysis (TGA) in which the probe molecule (gas or liquid) is desorbed and the weight loss is recorded. Comparison with untreated sample (background) enables an estimate to be made of both the quantity and strength of the acidic sites ${ }^{[96,102]}$.

\subsubsection{Acidity characterisation of the geopolymer catalysts}

In this work, qualitative characterisation of the surface acidity of the synthesised geopolymer catalysts was made by FTIR spectroscopy of adsorbed pyridine, while quantitative analysis of the total acidity was determined from the TGA profile of desorbed pyridine. The procedures of both experiments are described below. 
Adsorption/desorption of pyridine on the catalyst surface was carried out using a Micromeritics VacPrep061 sample degassing system. $0.1 \mathrm{~g}$ of the $\mathrm{H}$-form of the catalyst was degassed at $250{ }^{\circ} \mathrm{C}$ for $12 \mathrm{hr}$. at $200 \mathrm{mTorr}$ vacuum. The $\mathrm{H}$-form of the catalysts was prepared as described in section 2.1 .2 by heating the $\mathrm{NH}_{4}{ }^{+}$-geopolymer to different temperatures. In this case the solid was removed from the oven and immediately transferred to the degasser. After degassing, the temperature was set to $150{ }^{\circ} \mathrm{C}$ and the sample was exposed to pyridine $(100 \mu \mathrm{l})$ for $1 \mathrm{hr}$. The physisorbed pyridine was then desorbed at $100{ }^{\circ} \mathrm{C}$ for $1 \mathrm{hr}$. under the same vacuum conditions, and the sample was then removed from the degasser for FTIR and TGA measurements. The FTIR spectra were acquired as described in section 2.2.5. The resulting spectrum for each solid was compared with a background sample (without pyridine) that had been prepared and handled in exactly the same way.

Thermogravimetric analysis (TGA) of the pyridine desorption was obtained using a Shimadzu TGA-50 thermal analyser at a heating rate of $10{ }^{\circ} \mathrm{C} \min ^{-1}$ up to $800{ }^{\circ} \mathrm{C}$ in flowing air $\left(50 \mathrm{ml} \mathrm{min}^{-1}\right)$. The gradual weight loss of the sample with pyridine was recorded and compared with a background sample. The difference in the weight loss between the sample with and without pyridine indicated the total concentration of the acid sites in the sample.

\subsubsection{Porosimetry measurements}

The surface and textural characteristics of a solid catalyst, including the surface area and porous features are crucial factors in heterogeneous catalysis, since they have a direct influence on the catalyst reactivity and thus the reaction kinetics. Typically, a solid catalyst should possess a high surface area which in some cases equates to a higher density of active sites and increased efficiency, especially when the reaction occurs at the surface or when an active catalyst is used as a support. The pore volume and its diameter are also critical features in a solid catalyst that controls the path of the reactant and products into and away from the active sites. Pore volumes are largely responsible for the deactivation or inactivity of a catalyst. This is particularly important in microporous zeolites which show poor reactivity when dealing with bulk molecules despite their other excellent features (section 1.3.1). 


\subsubsection{Adsorption isotherms $\left(\mathbf{N}_{2}\right.$ isotherms)}

The surface area and the pore volume of a solid is usually measured by determining the physical adsorption (physisorption) of an inert gas $\mathrm{N}_{2}, \mathrm{Ar}$, and $\mathrm{Kr}$ on the solid surface at low temperature, typically that of $\mathrm{N}_{2}$ at the boiling point of liquid $\mathrm{N}_{2}(77 \mathrm{~K})$. Physisorption occurs due to Vander Waal's weak attractive forces between the gas or liquid molecules with the surface of the solid, while the chemisorption results from the formation of strong chemical bonds. Physisorption of $\mathrm{N}_{2}$ (adsorbate) on the surface of a solid (adsorbent) is expressed by an adsorption isotherm; the relation between the volume of the gas adsorbed (adsorbate) and the relative pressure $\left(p / p^{o}\right)$, where $p$ is the gas pressure and $p^{o}$ is the saturation vapour pressure at the temperature of the vessel containing the solid, at a relative pressure range between 0 and $1\left(0 \leq p / p^{o} \leq 1\right)$.

Adsorption isotherms are classified by the International Union of Pure and Applied Chemistry (IUPAC) into six main types ${ }^{[103]}$, (figure 2.6a). The type of the isotherm depends on whether the material is porous or not, and is related to the size of the pores. Porous materials are classified into three categories based on their pore diameter $\left(D_{\text {pore }}\right)$; Microporous ( $\left.D_{\text {pore }} \leq 2 \mathrm{~nm}\right)$, Mesoporous ( $\left.2 \mathrm{~nm} \leq D_{\text {pore }} \leq 50 \mathrm{~nm}\right)$, and Macroporous (50 $\left.\mathrm{nm} \leq D_{\text {pore }}\right)^{[103]}$.

The Type I isotherm is related to a microporous adsorbent with monolayers (a few molecular layers). The gas uptake is hindered and stabilises at high pressure by the micropore volume. Type II isotherms are related with a nonporous or macroporous adsorbent with unlimited gas uptake at the high pressure range. The point (B) indicates the completion of the monolayer coverage. Type III and V isotherms are not common and mostly result from weak adsorptive-adsorbent interactions. Mesoporous materials exhibit type IV isotherms which are related to type II isotherms, but with a distinctive hysteresis loop (figure 2.6b) which results from capillary condensation of the adsorbate inside the mesopores of the adsorbent. Type VI isotherms show stepwise multilayer adsorption and are usually associated with adsorption on nonporous materials with uniform surfaces, e.g. adsorption of $\mathrm{Kr}$ on nonporous graphitised carbon black. 

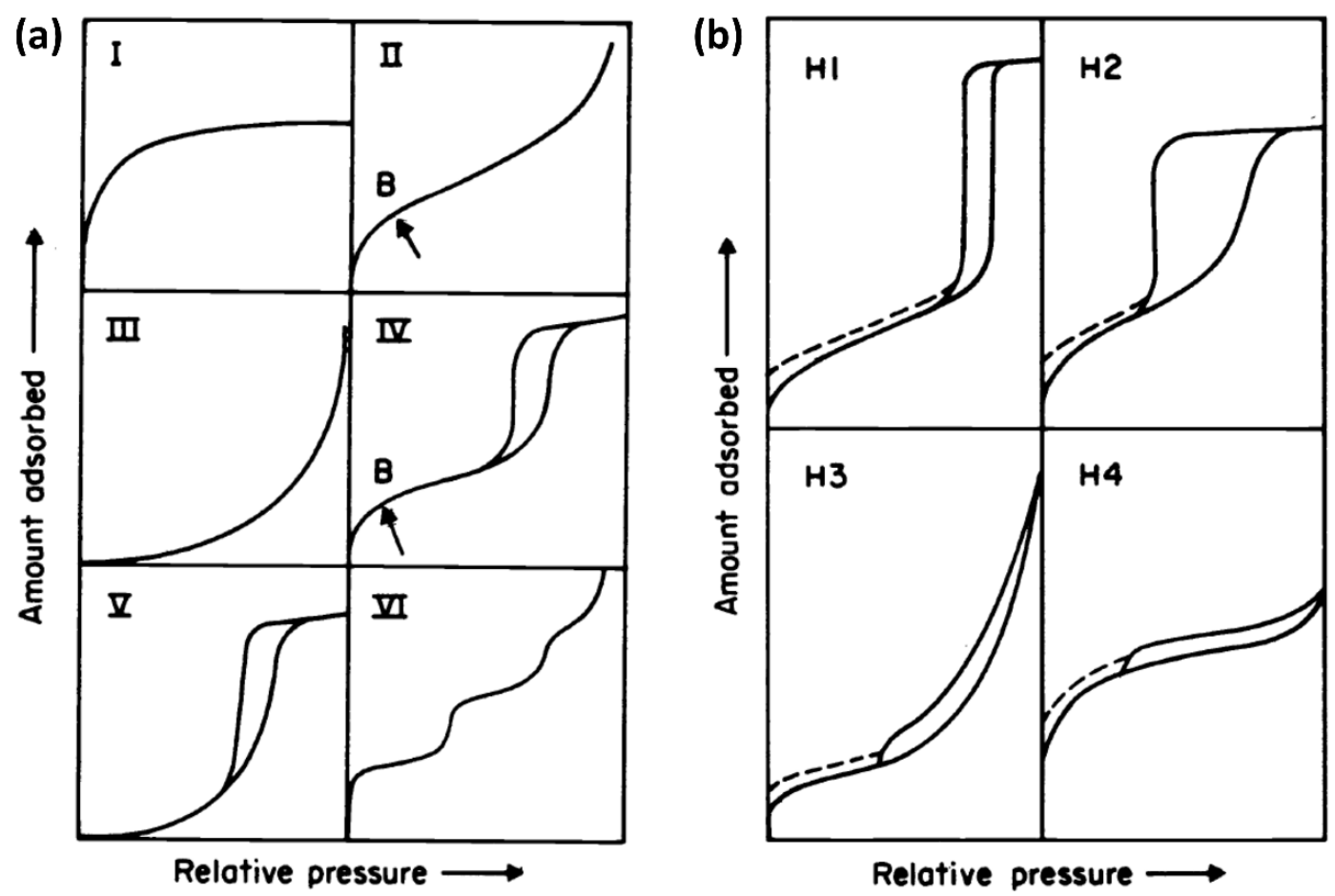

Figure 2.6 Types of physisorption isotherms (adsorption-desorption) (a), and the hysteresis loops associated with physisorption isotherm type IV (b) ${ }^{[103]}$.

The type of hysteresis loop (figure 2.6b) associated with type IV isotherm provides further insight about the pore shapes. Type $\mathrm{H} 1$ loops are obtained from porous materials that consist of agglomerates and have well-defined cylindrical pores with a narrow size distribution. Type $\mathrm{H} 2$ hysteresis loops, on the other hand, are common in porous glasses and inorganic gels where the pore shape and distribution is not well defined. Both hysteresis loops $\mathrm{H} 3$ and $\mathrm{H} 4$ are associated with porous materials consisting of aggregates forming slit-like pores. However, H4 hysteresis loops are usually related to narrower pores and their similarity to type I isotherms indicates the presence of microporosity.

\subsubsection{Models for adsorption isotherms interpretation}

Several methods have been developed to measure the surface area (S) and pore dimensions from the adsorption isotherms, the simplest of which is described by Langmuir equation (1):

$n=c p /[1+(c p)]$ 
Where $n$ is the volume of the adsorbed gas (monolayer), $c$ is a constant, and $p$ is the gas pressure. The main assumptions are; the adsorbed molecules only form a monolayer, the energy of adsorption on the surface of the solid is uniform, and there are no adsorbent-adsorbent interactions ${ }^{[104]}$. Although these assumptions have been shown to be untrue, the Langmuir isotherm still provides reasonable results when applied to chemisorption or physisorption Type I (figure 2.6). This is because the non-uniformity of the surface energy decreases the enthalpy of adsorption while the adsorbentadsorbent interaction increases it, thus the impact of these two assumptions is cancelled.

However, Langmuir theory fails to describe the multilayer formation which is common at low temperatures. In addition, if the material is mesoporous, multilayer formation is usually followed by capillary condensation in which the adsorbed gas condenses to a liquid-like phase in the mesopores (figure 2.7).

Brunauer-Emmett-Teller (BET) theory, introduced in 1938, provides a model for multilayer physisorption with the assumptions; the gas molecules are adsorbed on a solid in layers (interaction between these layers is ignored), and the Langmuir isotherm can be applied for each layer ${ }^{[105]}$. The BET equation is shown below (equation 2):

$p / V_{a d s}\left(p^{o}-p\right)=\left(1 / V_{m}\right)+\left[(c-1) p /\left(V_{m} c p^{o}\right)\right]$

Where $p$ is the gas pressure, $V_{a d s}$ is the total volume of the adsorbed gas, $p^{o}$ is the saturation vapour pressure, $V_{m}$ is the volume of the adsorbed gas for monolayer, and $c$ is a constant. Plotting the first half of the equation vs $\left(p / p^{o}\right)$ produces a linear plot in the range $0.05-0.3$ with a slope $=c-1 / V_{m} c$ and the intercept $=1 / V_{m} c$. From these two equations the value of $V_{m}$ is obtained. Taking into account the area of $\mathrm{N}_{2}=16.2 \AA^{2}$, the surface area of the solid is obtained and usually expressed as specific surface area $\left(S_{B E T}\right.$ $\left.\mathrm{m}^{2} / \mathrm{g}\right)$. 


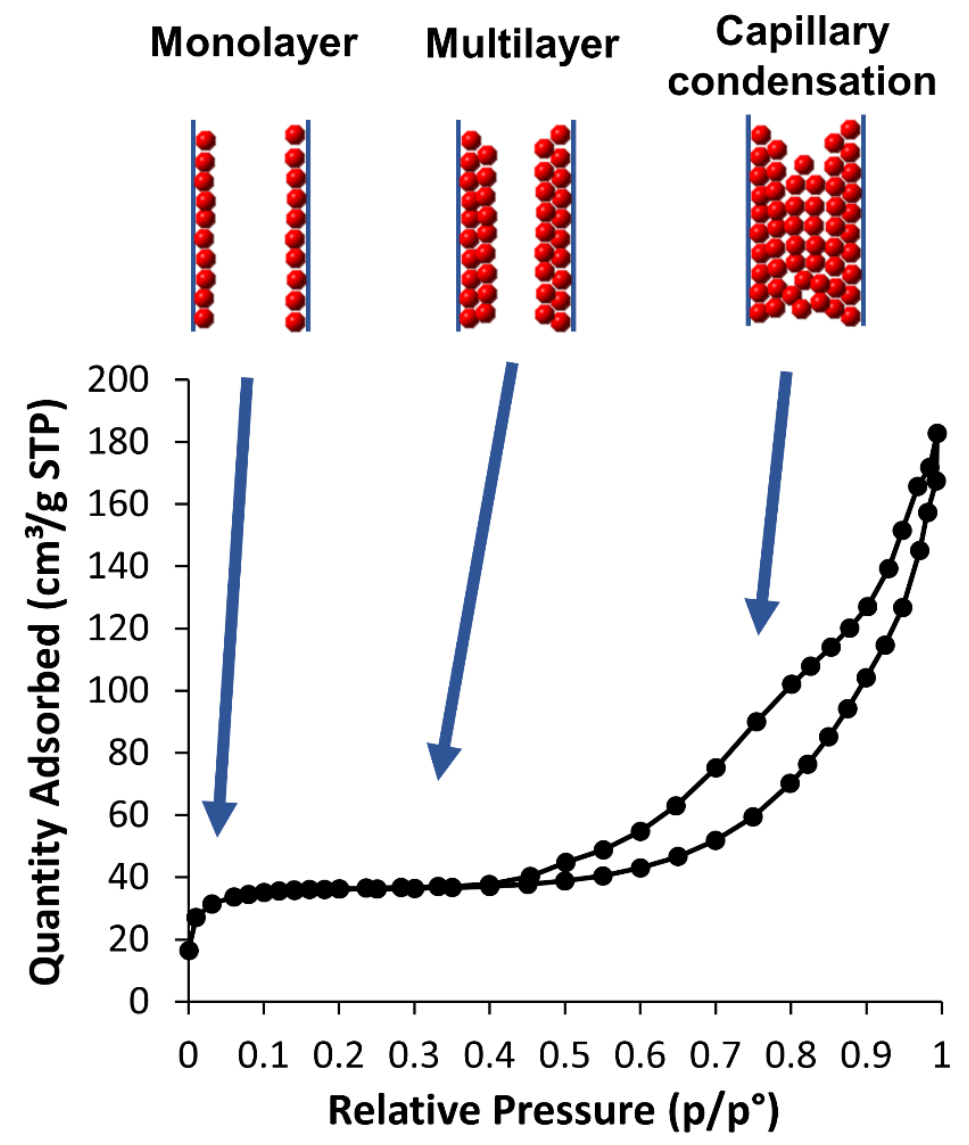

Figure 2.7 Stages of gas physisorption on a mesoporous solid.

Although BET theory is widely used for surface area measurements ${ }^{[89,105]}$, it fails to measurement the volume of the micropores because it ignores the surface energy inhomogeneity, which means the higher energy sites on the surface will be filled faster. Therefore, the BET plot is non-linear at relative pressures less than 0.05, which is the area usually associated with the micropore filling. A common method for measuring the micropore volume is the $t$-plot.

The $t$-plot is a plot of $V_{a d s}$ vs the thickness of the multilayers (t) at each point of $p / p^{o}$. The plot is linear in the range of monolayer to capillary condensation $\left(\sim 0.05 \leq p / p^{o} \leq\right.$ $\sim 0.5)$. The slope of the linear part of the plot represents the external surface area $\left(S_{\text {ext }}\right)$, the area of all the pores and cavities apart from the micropores which do not contribute to the formation of multilayers. Thus the micropore area $\left(S_{\text {micro }}\right)$ is obtained from $S_{\text {micro }}$ $=S_{B E T}-S_{\text {ext }}$. The micropore volume is measured from the intercept of the linear extension of the plot as shown in figure 2.8. t-plot method can also be applied to mesopore volume measurements ${ }^{[106]}$. 


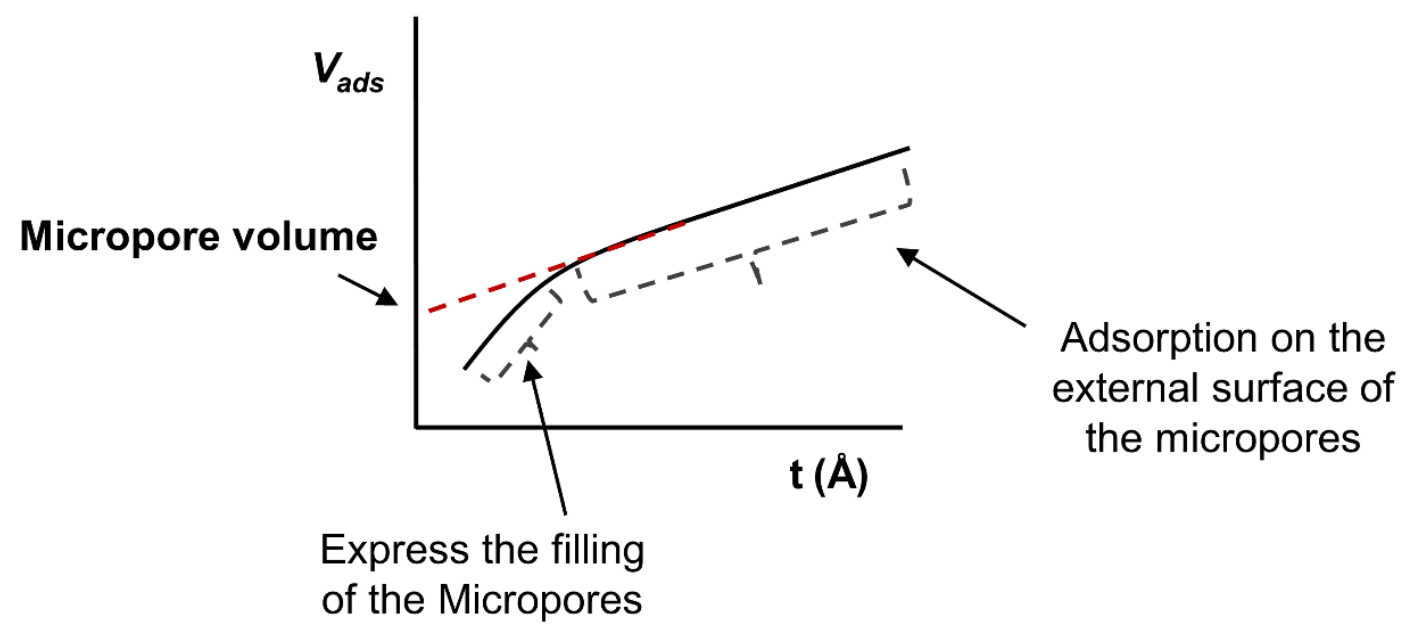

Figure 2.8 Illustration of the $t$-plot method.

The mesopore radius and volume are usually determined from the correlation between the pore diameter and the condensation pressure in the pore, assuming cylindrical pore shape. This relation is given by Kelvin as shown in equation (3) below:

$\operatorname{In}\left(p / p^{o}\right)=(\mathrm{V} / \mathrm{RT})\left(2 \sigma \cos \boldsymbol{\theta} / \mathrm{r}_{\mathrm{k}}\right) \ldots(3)$

Where $\mathrm{V}$ is the molar volume of the liquid adsorbate, $\mathrm{R}$ is the gas constant, $\mathrm{T}$ is temperature, $\sigma$ is surface tension of the liquid adsorbate, $\boldsymbol{\theta}$ is the angle between the adsorbate and the adsorption layer on the wall of the pore $(\boldsymbol{\theta}=0), \mathrm{r}_{\mathrm{k}}$ is the Kelvin critical radius of the pore ${ }^{[3]}$. Kelvin theory excludes the thickness of the layers formed prior to capillary condensation. A modified version of the Kelvin equation was derived by Barrett-Joyner-Halenda (the BJH method), in which the pore size $\left(\mathrm{r}_{\mathrm{p}}\right)$ is taken as the sum of the Kelvin radius $\left(\mathrm{r}_{\mathrm{k}}\right)$ and the thickness of the physisorbed layers ( $\mathrm{t}$ ) (equation (4)). The BJH method also allows he pore size distribution to be determined, provided the desorption branch is measured ${ }^{[3]}$.

$r_{p}=r_{k}+t \ldots(4)$

\subsubsection{Geopolymer porosity measurements}

In this work, the $\mathrm{N}_{2}$ adsorption-desorption isotherms were determined using a Micromeritics ASAP 2010 instrument. All the samples were degassed at $110{ }^{\circ} \mathrm{C}$ down to 3 mTorr vacuum using the instrument degassing system. The specific surface area $\left(S_{B E T}\right)$ was measured by the Brunauer-Emmett-Teller $(\mathrm{BET})$ method over a $\mathrm{p} / \mathrm{p}^{\mathrm{o}}$ range 
of $0.05-0.3$. The total pore volume ( $\left.V_{\text {total }}\right)$ was measured by single point adsorption at $\mathrm{p} / \mathrm{p}^{\mathrm{o}}=0.995$. Barrett-Joyner-Halenda $(\mathrm{BJH})$ method was used to measure the mesopore volume $\left(V_{\text {meso }}\right)$, the pore diameters $\left(D_{\text {pore }}\right)$, and the pore size distribution.

\subsubsection{Particle size distribution}

The particle size of a solid catalyst and its surface area are interrelated quantities and are crucial parameters in heterogeneous catalysis. In addition, the particle size affects the mass and heat transfer parameters (see section 2.3.3), thus affecting the catalytic reaction kinetics. Several methods can be employed to measure the particle size of a solid material; for instance, SEM provides an estimate of the particle diameters. The size of solid crystallites can be measured by X-ray line broadening and small angle Xray scattering (SAXS), these techniques are described elsewhere ${ }^{[98,107]}$.

Another common technique is based on light scattering. Static laser diffraction is used for particles in the micro range, while the dynamic light scattering is used for smaller particles at the nano scale. In the latter method, the sample is illuminated with a laser beam, and the scattered light is collected by a detector placed at a specific angle. Due to Brownian motion of sub-micron particles, collisions between these particles change the intensity of the scattered light, from which the particle size is determined ${ }^{[108]}$.

In the static light scattering method, the scattered light is collected by a detector at a wide range of angles. From the intensity and the angle of the scattered light the particle size is determined, since the larger the particle the smaller the scattering angle and the higher the intensity. In this method, Mie theory is applied, where the particle is assumed to be spherical with a known refractive index ${ }^{[108]}$.

In this work, the particle size distribution of the synthesised geopolymers was estimated by laser diffraction using a Malvern Mastersizer 2000 instrument. The refractive index of the geopolymer was taken as 1.55 (similar to an aluminosilicate kaolin clay). The geopolymer powder was suspended in distilled water. The results were taken as the average of three measurements. Even though geopolymer particles are not spherical (rounded particles) and their exact refractive index is unknown, this technique is still widely acceptable and provides a reasonable estimation of their particle size distribution. 


\subsection{Catalytic reactions}

\subsubsection{Reaction system}

All the catalytic reactions in this study were performed in liquid phase systems under atmospheric pressure in a magnetically stirred 50-ml two-necked round bottom flask equipped with a reflux condenser and placed in a thermostatic bath using Silicon oil.

In each run, the solvent and the reactant were mixed together in a specific ratio at a specific temperature followed by addition of the catalyst. The time at which the reaction reached the desired temperature was taken as the start of the reaction. The catalyst was the $\mathrm{NH}_{4}{ }^{+}$ion-exchanged form of the geopolymer that had been thermally treated at different temperatures for $15 \mathrm{~min}$. before being added to the reaction mixture. It should be noted that for each reaction, the catalyst was removed from the oven and immediately added straight to the reaction vessel. In this way, the acid form of the geopoylmer was formed by decomposing the $\mathrm{NH}_{4}{ }^{+}$simultaneously with activation of the catalyst by removal of the physisorbed species.

More details regarding the reaction time, the molar ratios of the reactants to the catalyst, reaction temperatures, the temperatures of the catalyst thermal treatments and the procedure for testing the reusability of the catalysts will be described in each subsequent chapter.

\subsubsection{Analysis of the reaction outcomes}

The outcome of each reaction was analysed by sampling over the course of the reaction. About $100 \mu \mathrm{l}$ aliquots were taken from the reaction vessel at specific time intervals using a syringe connected to a micro-filter. The samples were analysed with a Shimadzu QP2010-Plus gas chromatograph (Shimadzu, Kyoto, Japan) fitted with an RXI 5SilMS column ( $30 \mathrm{~m}$ x $0.25 \mathrm{~mm}$ i.d. x $0.25 \mu \mathrm{m}$ film thickness, Restek, Bellefonte, PA) attached to an electron impact mass spectrometer operating at $70 \mathrm{eV}$ in positive ion mode, scanning from $\mathrm{m} / \mathrm{z} 42-600$ every $0.3 \mathrm{sec}$.

$1 \mu \mathrm{l}$ samples were introduced using an AOC - 20i auto-sampler with a split injection (20:1) at an injector temperature of $270{ }^{\circ} \mathrm{C}$ using $\mathrm{He}$ as the carrier gas at a linear velocity of $43.4 \mathrm{~cm} / \mathrm{s}(1.38 \mathrm{ml} / \mathrm{min})$ at constant flow. Each sample was injected at an initial oven 
temperature of $50{ }^{\circ} \mathrm{C}$ for $2 \mathrm{~min}$, followed by a ramp at $20{ }^{\circ} \mathrm{C} / \mathrm{min}$ to $300{ }^{\circ} \mathrm{C}$ with a final hold for $5 \mathrm{~min}$. The reaction products were identified by comparison of the TIC fragmentation mass spectra with those contained in the NIST11 library with a similarity score of $>85 \%$ in at least two of three replicates. The identity of each compound in the reaction mixture was then confirmed by comparison of the fragmentation pattern and retention time with that of authentic standards (positive identification; Sigma-Aldrich, Castle hill, Australia or AK Scientific, Union City, CA).

Quantitative analysis was performed using conventionally-determined calibration curves in which five standards were prepared from the reactant and the product which were analysed with each run. Each reaction was repeated at least three times for statistical purposes and the reproducibility is expressed as the standard error (SE), described in equation (5).

$\mathrm{SE}=($ standard deviation $) / \sqrt{ }($ number of replicates $) \ldots(5)$

\subsubsection{Catalytic activity}

Investigating a new heterogeneous catalyst requires a precise characterisation not only of its chemical and structural properties such as acidity, porosity, etc., but also its catalytic activity ${ }^{[109]}$. This should be done following recommended standard methods to provide a valid comparison with other existing catalysts. The catalytic activity measurements such as conversion, selectivity and reaction rates should also be free of reaction defects such as mass and heat transfer limitations. The ways of expressing the catalytic activity and the common models for evaluating the presence or absence of mass and heat transfer limitations are described below.

\subsubsection{Conversion and selectivity}

The activity of a catalyst is related to its ability to convert the reactant (conversion) to the desired product (selectivity). Based on the IUPAC recommendations [110], conversion, selectivity, and the yield of the desired product were measured as follows:

Conversion $(\%)=\frac{\text { amount of reactant converted (mole) }}{\text { amount of reactant fed (mole) }} \times 100 \% \ldots$ (6)

Where the amount converted (mole) $=$ amount fed (mole) - amount remaining (mole) . 
Selectivity $(\%)=\frac{\text { amount of a particular product formed }}{\text { amount of reactant converted }} \times 100 \% \ldots$

Yield $(\%)=\frac{\text { amount of a particular product formed }}{\text { amount of reactant fed }} \times 100 \% \ldots$ (8)

Equation $(8)$ can be expressed as Yield $(\%)=$ conversion $(\%)$ x selectivity $(\%)$.

\subsubsection{Reaction rate}

Reaction rate of conversion or formation in a heterogeneous catalytic reaction is usually normalised to the amount of the solid catalyst being used, which can be expressed as mass, volume or surface area ${ }^{[105,110]}$. Thus, the reaction rate can be expressed in three ways as described below.

Specific rate $(\mathrm{mol} / \mathrm{g} . \mathrm{s})=\frac{\text { amount of reactant converted }}{\text { mass of catalyst } \mathrm{x} \text { reaction time }}$

Volumetric rate $\left(\mathrm{mol} / \mathrm{cm}^{3} . \mathrm{s}\right)=\frac{\text { amount of reactant converted }}{\text { volume of catalyst } \mathrm{x} \text { reaction time }} \ldots$

Where the volume of the catalyst is related to the volume of the catalyst particles, excluding the interparticle volume.

Areal rate $\left(\mathrm{mol} / \mathrm{cm}^{2} . \mathrm{s}\right)=\frac{\text { amount of reactant converted }}{\text { surface area of catalyst } \mathrm{x} \text { reaction time }}$

The amount of reactant converted can be replaced with the amount of a particular product formed, thereby obtaining the rate of its formation. Therefore, one should specify the way in which these measurements were calculated. In this study, the reaction rates were measured as specific rates of conversion, unless otherwise specified.

The Langmuir-Hinshelwood kinetic model, which is the most widely applied model to express the kinetics of a heterogeneous catalytic reaction ${ }^{[111]}$, was used in this study to give the reaction rate constants. The activation energies for the catalytic reactions studied here were obtained from the slopes of the Arrhenius plots. 


\subsubsection{Turn over numbers}

When the reaction rate is normalised to the active component of the catalyst, particularly the number of active sites, this is called the specific activity or turn over number (TON) ${ }^{[105]}$. Where the TON is expressed per time unit (usually seconds), this is then referred to as the turnover frequency (TOF). The TON is defined as the number of molecules reacting per active site, which represents the number of revolutions of catalytic cycles that a catalyst will undergo, and hence it gives an indication of the catalyst lifetime $^{[110,112]}$. The difficulty in measuring the TON or TOF lies mainly in determining the number of active sites. This is usually an approximation made by using some standard method for measuring the active sites such as chemisorption of a probe molecule; therefore turn over numbers are approximate values of the overall catalyst activity $[105,112]$. Despite this, TOF values have advantages for comparing the performance of a new catalyst with other known catalysts.

In this work, the TON and TOF values were measured as follows ${ }^{[110,113]}$ :

TON $=\frac{\text { number of moles of reactant converted per gram of catalyst. }}{\text { number of moles of the acidic sites per gram of catalyst. }} \ldots$

TOF $\left(\mathrm{s}^{-1}\right)=\frac{\mathrm{TON}}{\text { reaction time }}$

\subsubsection{Models to evaluate the mass and heat transfer limitations}

In a heterogeneous catalytic reaction, it is very important to ensure that the kinetic data were obtained from a kinetically-controlled system free of mass and heat transfer limitations. This can be achieved by the use of a valid evaluation of the impact of these factors using one of the commonly applied models.

The heat transfer limitation in liquid phase reaction systems can be excluded due to the high heat capacity and thermal conductivity of the liquid phase compared to the gas phase ${ }^{[105,114]}$.

On the other hand, when a porous catalyst is used, the diffusional limitations, whether external (interphase, between the liquid and the solid phases) or, more importantly, internal (intraphase, within the pores of the catalyst) can alter the kinetics of the catalytic system. The impact of the external mass transfer can be 
eliminated by ensuring proper mixing of the reaction solution, by increasing the stirring speed of the reaction until no further increase in the reaction rate is observed $^{[105]}$. In the present work, a stirring speed of $700 \mathrm{rpm}$ was used, chosen to be sufficient to avoid the external mass transfer limitations for the tested reactions.

Therefore, in liquid phase "slurry" reaction systems with sufficient mixing, internal mass transfer limitation is the main factor that may affect the reaction kinetics. Several models have been suggested to evaluate the impact of the internal mass diffusion limitations. Some of the commonly applied models are briefly described below.

The Koros-Nowak test assumes that in a kinetically controlled regime the reaction rate is proportional to the concentration of the active sites. In this method, the concentration of the active sites is diluted and if the TOF remains constant, no internal mass limitation is present ${ }^{[115]}$. A version of the Koros-Nowak method developed by Madon and Boudart ${ }^{[116]}$ states that if the Koros-Nowak test is obeyed at two different temperatures, the reaction data are free of heat and mass transport limitations and other artifacts such as poisoning.

Another common method is the Thiele modulus $(\phi)$, in which the mass transfer limitation is estimated from the ratio between the surface reaction rate and the rate of diffusion of the reactant within the pores of the catalyst. In this method, a value of $\phi$ is calculated, from which an effectiveness factor $(\boldsymbol{\eta})$ is determined with values between 0 to 1 . The absence of mass transfer limitation is indicated when $\boldsymbol{\eta}$ is close to $1^{[105]}$. This method is described in detail elsewhere ${ }^{[105,114]}$.

Another widely applied test is the Weisz-Prater criterion ( $\left.\phi_{\text {W.P. }}\right)$, (equation (14)), which also represents the ratio of the reaction rate and the diffusion rate of the reactants within the pores. According to this criterion, if the value of the dimensionless $\phi_{\text {W.P }}$ parameter $<0.3$ for a reaction of $2^{\text {nd }}$ order or less, internal mass diffusion limitations can be eliminated ${ }^{[114,117]}$, whereas a value of $\phi_{W . P} p>$ 6 indicates significant diffusional control. 
In this study, the internal mass transfer limitation was evaluated by the $\phi_{\mathrm{W} . \mathrm{P}}$ criterion as described below (equation 14), paying attention to the units:

$\phi$ W.P. $=\frac{r_{\mathrm{a}} R_{\mathrm{p}}^{2}}{\mathrm{C}_{\mathrm{a}} \mathrm{D}_{\mathrm{eff}}} \ldots$

Where $r_{a}$ is the reaction rate per catalyst volume ( $\mathrm{mol}$ reactant $\left./ \mathrm{cm}^{3} . \mathrm{s}\right), \mathrm{Rp}$ is the catalyst particle radius $(\mathrm{cm})$ and $\mathrm{Ca}$ is the concentration of the reactant on the surface of the catalyst $\left(\mathrm{mol} / \mathrm{cm}^{3}\right)$.

In liquid phase systems, this can be considered as the bulk concentration if the external mass transfer limitations have been excluded by proper mixing ${ }^{[14]}$.

$D_{\text {eff }}$ is the effective diffusivity $\left(\mathrm{cm}^{2} / \mathrm{s}\right)$ which was measured in this work by the Ternan method (equation 15):

$D_{\text {eff }}=D_{b} \frac{(1-\lambda)^{2}}{1+P \lambda}$

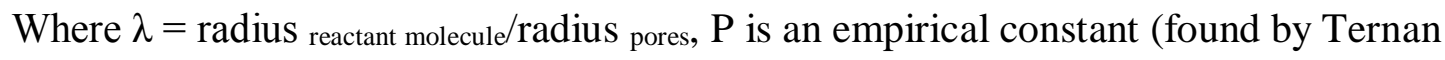
to be 16.3$)^{[117]}$ and $\mathrm{Db}$ is the bulk diffusivity $\left(\mathrm{cm}^{2} / \mathrm{s}\right)$ which was measured by the method of Wilke and Chang ${ }^{[114,117-118]}$ (equation 16):

$\mathrm{D}_{\mathrm{b}}=1.1728 \times 10^{-16} \frac{\mathrm{T} \sqrt{\mathrm{XM}_{2}}}{\eta_{2} \mathrm{~V}_{1}^{0.6}} \ldots$

Where $\mathrm{T}$ is the temperature $(\mathrm{K}), \mathcal{X}$ is the association constant of the solvent, $\mathrm{M}_{2}$ is the molar mass of the solvent $(\mathrm{g} / \mathrm{mol}), \mathrm{n}_{2}$ is the viscosity of the solvent at the reaction temperature (Pa.s) ${ }^{[19]}$ and $\mathrm{V}_{1}$ is the molar volume of the reactant at its normal boiling point $\left(\mathrm{m}^{3} / \mathrm{kmol}\right)$. This was determined by the method of Grain ${ }^{[120]}$ (equation 17):

$\mathrm{V}_{1}=\frac{\mathrm{M}_{1}}{\mathrm{~d}}\left(3-2 \frac{\mathrm{T}}{\mathrm{Tb}}\right)^{0.31}$

Where $\mathrm{M}_{1}$ is the molar mass of the reactant $(\mathrm{g} / \mathrm{mol}), \mathrm{D}$ is the density of the reactant $\left(\mathrm{g} / \mathrm{cm}^{3}\right)$ at temperature $\mathrm{T}(\mathrm{K}), \mathrm{Tb}$ is the normal boiling point of the reactant $(\mathrm{K})$. 


\section{Chapter 3}

\section{Activity of geopolymer catalysts in}

Beckmann rearrangement reaction

\subsection{Introduction}

The Beckmann rearrangement is a ubiquitous organic reaction vital to many industrially important organic syntheses. One such reaction is the production of $\varepsilon$-caprolactam, the precursor of polyamide-6 (nylon-6), which is produced in vast amounts; in 2012 alone, five million metric tonnes of caprolactam were produced ${ }^{[121]}$. The industrial synthesis of caprolactam is by a one-pot ammoximation of cyclohexanone, followed by the Beckmann rearrangement of the cyclohexanone oxime using concentrated sulphuric acid and/or oleum as the catalyst and solvent as shown in Figure $3.1^{[122]}$.

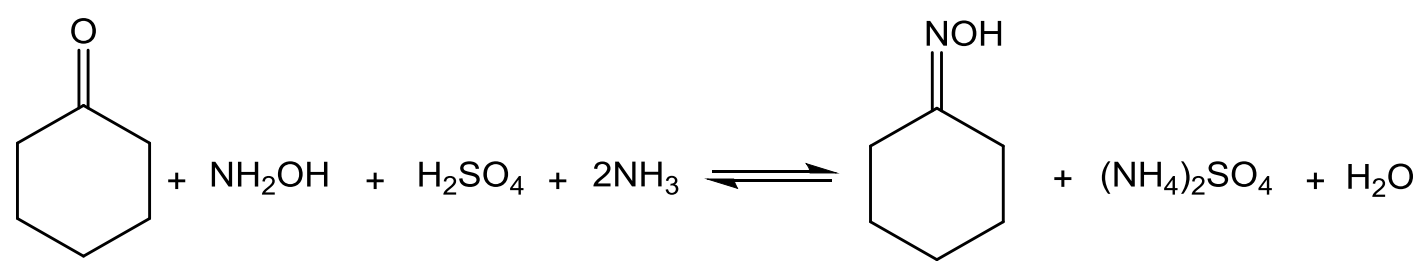

cyclohexanone

cyclohexanone oxime

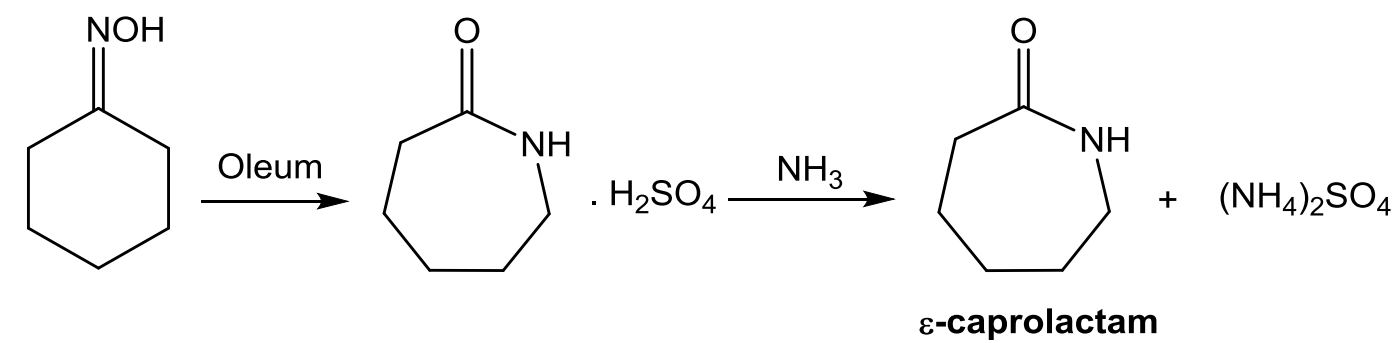

Figure 3.1 Industrial production of $\varepsilon$-caprolactam ${ }^{[122]}$. 
Each tonne of caprolactam produced in this process produces two tonnes of ammonium sulfate waste, formed by neutralisation of the acid with ammonia ${ }^{[12]}$. Generation of such large amounts of waste presents serious environmental problems and expense involved in its treatment and disposal. Therefore, considerable research effort has been expended in the development of environmentally friendly alternatives to catalyse Beckmann rearrangement reactions ${ }^{[123]}$. Several of the heterogeneous catalysts employed for the gas phase Beckmann rearrangement of cyclohexanone oxime to $\varepsilon$ caprolactam during the last three decades include zeolites (mainly high-silicon MFI framework structures [99a, 124] and nanosheets ${ }^{[99 b]}$, molecular sieves ${ }^{[125]}$ and functionalised oxides or mixed oxides ${ }^{[126]}$. Large-scale production of $\varepsilon$-caprolactam ( 90000 tonnes/year) by the Beckmann rearrangement in the gas phase was achieved in 2003 by the Sumitomo Corporation, Japan, using H-ZSM-5 zeolite as the catalyst [127].

However, such vapour-phase systems suffer the drawback of requiring high temperatures (often $>623 \mathrm{~K}$ ) which can also deactivate the catalyst by facilitating side reactions such as polymerisation, with a deleterious effect on the catalyst life time and reusability ${ }^{[121,128]}$. Although the reusability problem can be solved by introducing into the reaction system an additional fluidized-bed reactor for regenerating the catalyst while conducting the reaction ${ }^{[127]}$, such systems are energy consuming. Thus, for both economic and environmental reasons, interest has recently increased in the liquid-phase Beckmann rearrangement of cyclohexanone oxime. Various heterogeneous catalysts have been proposed for this purpose, including zeolites (mainly large pore Y and beta type zeolites $\left.{ }^{[129]}\right)$, molecular sieves ${ }^{[121,130]}$, mixed oxides ${ }^{[128 a, 131]}$, heteropolyacids ${ }^{[128 b]}$, Metal organic frameworks (MOFs) ${ }^{[128 c]}$ and ionic liquids ${ }^{[132]}$.

The nature and the location of the active sites responsible for catalysing the Beckmann rearrangement reaction are in some dispute in the literature. Some reports suggest that the rearrangement is catalysed by Bronsted acid sites located inside the pores of a ZSM5 zeolite catalyst ${ }^{[133]}$, whereas other studies have implicated the strong acidic bridging hydroxyl groups in the strong adsorption of the product to the catalyst, giving rise to its rapid deactivation ${ }^{[134]}$. However, a more recent general consensus is that the most likely active sites for this rearrangement are the non- or weakly acidic hydrogen-bonded silanol groups (silanol nests) and vicinal silanols located on the outer surface of the 
catalyst or just under the mouths of the pores ${ }^{[126-127]}$. Non-acidic silanol groups (isolated $\mathrm{Si}-\mathrm{OH})$ located on the outer surface of zeolite nanosheets have also been reported to be active sites for this reaction ${ }^{[99 b]}$. The mechanism of this reaction over solid acids has previously been suggested to involve the protonation of the oxygen on the hydroxyl group of the oxime as the first step, followed by alkyl migration, simultaneously with dehydration giving carbinium and nitrilium cations. Hydrolysis of the resulting cation by the liberated water forms the amide ${ }^{[135]}$. However, recent kinetic and spectroscopic studies have shown that the first step of the mechanism is more likely to involve the protonation of the nitrogen atom followed by a 1,2-H shift from the nitrogen to the oxygen atom of the oxime forming carbinium and nitrilium cations as shown in Figure $3.2^{[134-136]}$. The latter is considered as the key to the rearrangement reaction as it is the most energy-demanding step ${ }^{[129 a, 135]}$.

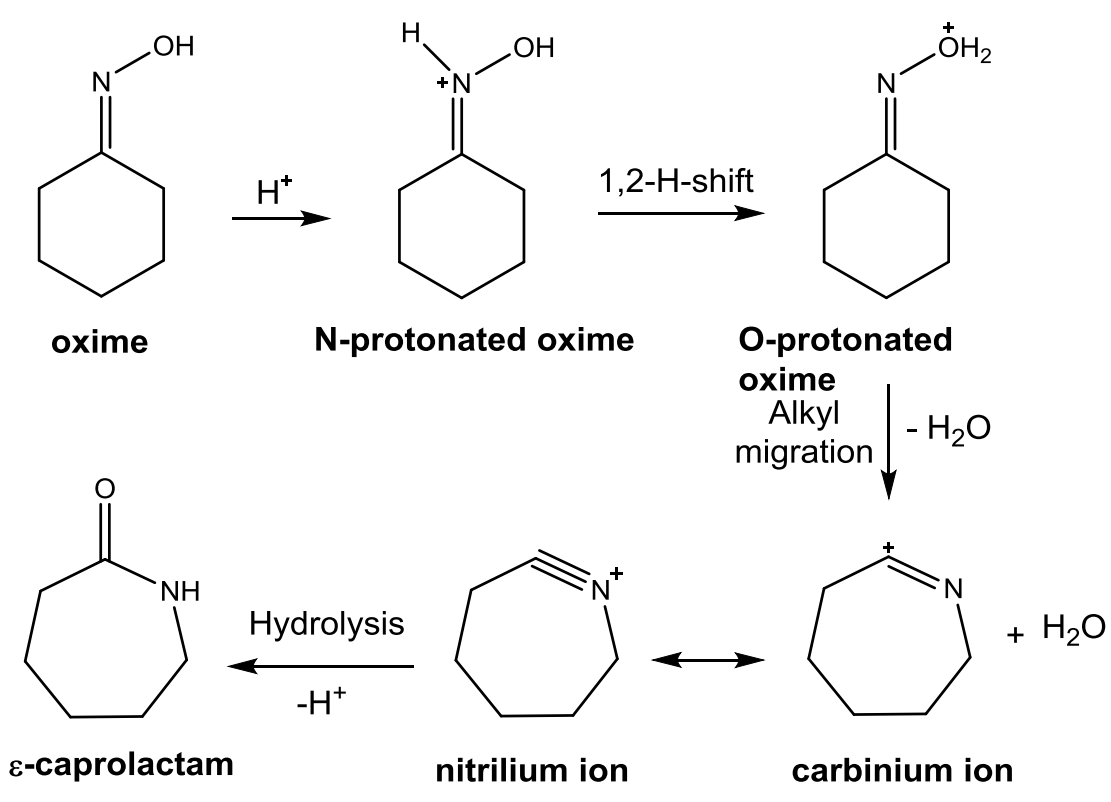

Figure 3.2 Reaction mechanism of Beckmann rearrangement of cyclohexanone oxime to $\varepsilon$-caprolactam (redrawn from ref. ${ }^{[134]}$ )

The solvent plays a crucial role in the liquid phase Beckmann rearrangement. Solvents with high polarity have been reported to show poor catalytic activity which is ascribed to the strong adsorption of the solvent on to the surface of the catalyst, thus preventing the reactant from reaching the active sites ${ }^{[129 a, 130 a, 135]}$. This suggests that non-polar solvents such as toluene should be preferable for this type of reaction, but no activity was observed when toluene was used, indicating the operation of factors other than solvent polarity. The solvent acts as a co-catalyst in which it interacts with the $\mathrm{N}$ - 
protonated oxime forming a five-membered ring complex (Figure 3.3) ${ }^{[129 a]}$. This complex favours the 1,2 $\mathrm{H}$-shift and thus accelerates the reaction. Therefore, the proton affinity of the solvent which is related to the dielectric constant is very important; in other words, a typical solvent for the liquid phase Beckmann rearrangement should have a moderate polarity and electron affinity.

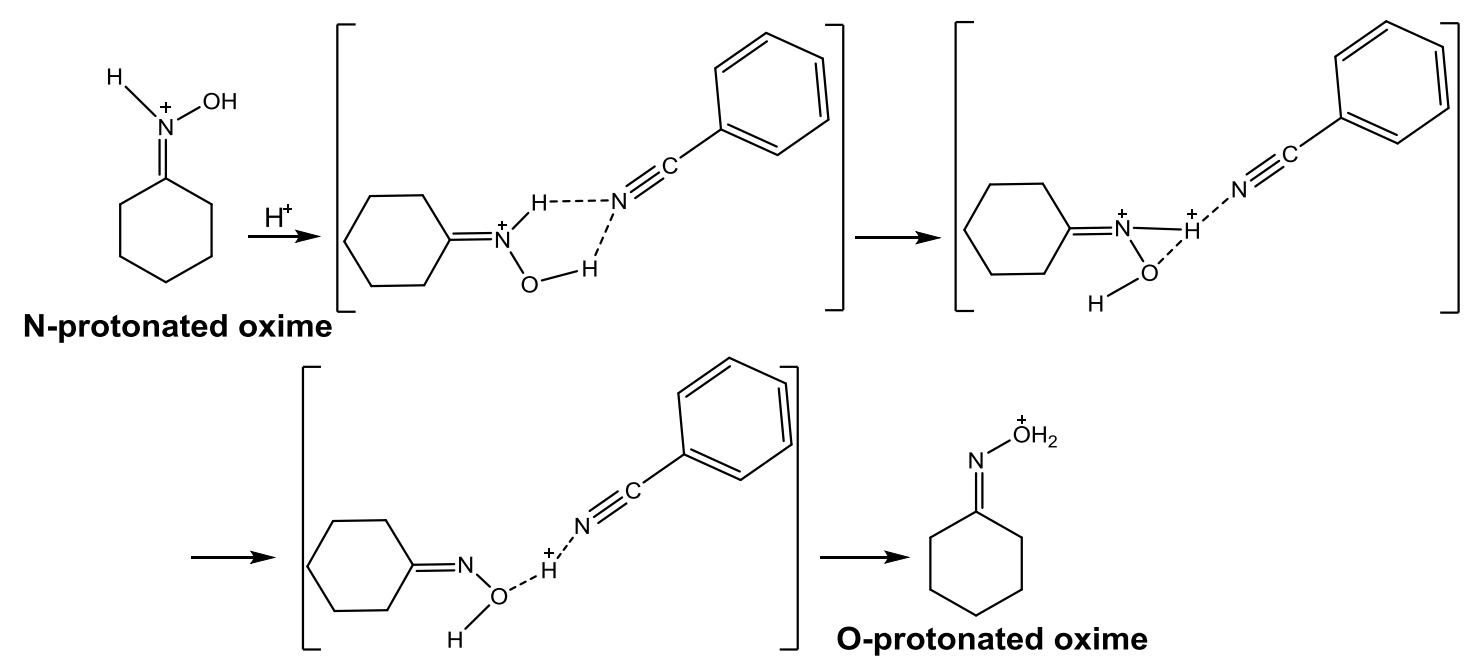

Figure 3.3 Role of the solvent $(\mathrm{PhCN})$ in the 1,2 $\mathrm{H}$-shift during Beckmann rearrangement of cyclohexanone oxime to $\varepsilon$-caprolactam (edited from ref ${ }^{[129 a]}$ ).

The application of solid catalysts in liquid-phase organic reactions in general, and the Beckmann rearrangement in particular, is very limited compared with vapour-phase systems ${ }^{[128]}$. This is due to the limitations associated with the reactive solid catalysts as described in chapter 1 .

In the present chapter, it is shown that geopolymers can be synthesised with chemical and textural properties suitable for very efficient catalysis of the liquid-phase Beckmann rearrangement of cyclohexanone oxime to $\varepsilon$-caprolactam. The nature of the active sites generated within the geopolymer framework was studied qualitatively and quantitatively. The effect of varying the geopolymer compositions on the textural and acidic properties of the catalysts was also studied and its influence on the catalytic activity was examined. Post-synthetic treatments to improve the catalytic performance of the prepared catalysts were also developed, and their performance over multiple reaction cycles evaluated. The performance of the geopolymer catalysts was compared 
with zeolites Y, HZSM-5 and with other reported catalysts in which the reaction was carried out under identical conditions.

\subsection{Experimental}

\subsubsection{The catalyst preparation}

The catalysts used in the model Beckmann rearrangement reaction were the $\mathrm{NH}_{4}{ }^{+}$ionexchanged form of the geopolymers, heated to $450{ }^{\circ} \mathrm{C}$ and held for $10 \mathrm{~min}$ prior to the reaction. The catalyst was removed from the oven and immediately added to the reaction vessel. The acidity measurements of the geopolymer catalysts tailored for the Beckmann rearrangement were carried out on $\mathrm{NH}_{4}{ }^{+}$-geopolymers heated to $450{ }^{\circ} \mathrm{C}$.

In this chapter, HZSM-5 and $\mathrm{Y}$ zeolites were also tested in the Beckmann rearrangement reaction under identical conditions for comparison purposes. Both the $\mathrm{NH}_{4}{ }^{+}$-ion exchanged zeolites were heated in the same manner as geopolymers (to 450 ${ }^{\circ} \mathrm{C}$ and held for $10 \mathrm{~min}$ ), heating details are described in section 2.1.2.

As described in section 2.1.3, some of the catalysts were subjected to post-synthetic treatments to improve their catalytic reactivity (see section 2.1.2). Of all the sequentially treated catalysts, only the Na-hiSi-Seq version was found to give improved catalytic performance for the Beckmann rearrangement reaction; for this reason, only this particular catalyst was studied in detail.

\subsubsection{Catalytic reaction}

The rearrangement of cyclohexanone oxime to $\varepsilon$-caprolactam was carried out in a magnetically stirred 50-ml two-necked round bottom flask equipped with a reflux condenser and placed in a thermostatic bath. In view of the known sensitivity of these reactions to moisture, some of these experiments were carried out under dry $\mathrm{N}_{2}$ using a Schlenk line. In a typical run $0.1 \mathrm{~g}$ of cyclohexanone oxime was dissolved in $20 \mathrm{ml}$ solvent (benzonitrile) and heated to $100{ }^{\circ} \mathrm{C}$ followed by the addition of $0.1 \pm 0.001 \mathrm{~g}$ of the catalyst and the reaction temperature was set at $130{ }^{\circ} \mathrm{C}$ under atmospheric pressure with $700 \mathrm{rpm}$ stirring speed for $5 \mathrm{hr}$. (wt.\% 1:200:1 respectively). Calibration curves covering the range of $0.044-0.009 \mathrm{mmol} / \mathrm{ml}$ of each reactant and product were used for quantitative analysis. 
Specifically in rearrangement reactions, the carbon mass balance is an important tool to monitor the reliability of the results. This was measured as follows ${ }^{[125 b]}$ :

Mass balance $(\%)=\frac{\text { sum of all products formed }+ \text { amount of oxime remained }}{\text { amount of oxime fed }} \times 100 \%$

All the other catalytic measurements were calculated as described in chapter 2, section 2.3.3.

To evaluate the reusability of the catalyst in this particular reaction, the catalyst was transferred quantitatively to a centrifuge tube after each reaction cycle and separated from the reaction solution. The solid catalysts were then washed twice with $2 \mathrm{ml}$ acetone which was combined and analysed by GC-MS to identify and quantify any adsorbed reactant or product on the surface of the catalyst; the amounts of these components (which was found to be $<7.00 \mathrm{wt}$. $\%$ of the starting material) were combined with those of the primary catalytic results. After washing with acetone, the catalyst was dried at $\sim 50{ }^{\circ} \mathrm{C}$ overnight then heated again to $450{ }^{\circ} \mathrm{C}$ for $10 \mathrm{~min}$ prior to the next reaction cycle. The structural stability of the spent geopolymer-based catalyst was evaluated by XRD and FTIR after five reaction cycles.

\subsection{Results and discussion}

\subsubsection{Catalyst characteristics}

XRD traces of the synthesised catalyst powders are shown in Figure 3.4. All the geopolymers show the broad background amorphous feature from $20-40^{\circ} 2 \theta$ typical of well-formed geopolymers on which are superimposed two sharp reflections arising from crystalline silica polymorphs quartz and cristobalite present as impurities in the original clay (Figure 3.4a and 3.4b).

Figure 3.4a shows the diffractogram of the initial clay. Dehydroxylation caused breakdown of the crystal structure of the clay forming an amorphous material (Figure $3.4 b$ ), which facilitates the geopolymerisation process. Traces of $\mathrm{Na}_{2} \mathrm{CO}_{3} \cdot \mathrm{H}_{2} \mathrm{O}$ are present in the synthesised geopolymers, arising from reaction of the $\mathrm{NaOH}$ with atmospheric $\mathrm{CO}_{2}$; this is most evident in the Na-hiSi sample (Figure 3.4f) which contains the highest $\mathrm{Na}_{2} \mathrm{O}$ content (Table 2.2). 
The XRD pattern of Na-hiSi-Seq (Figure 3.4g) shows a slight shift of the broad background hump that may be due to removal of some of the crystalline phases and Alrich debris during washing. The disappearance of the $\mathrm{Na}_{2} \mathrm{CO}_{3} \cdot \mathrm{H}_{2} \mathrm{O}$ from this sample is ascribed to the removal of most the Na cations from the geopolymer framework by ionexchange with $\mathrm{NH}_{4}{ }^{+}$.

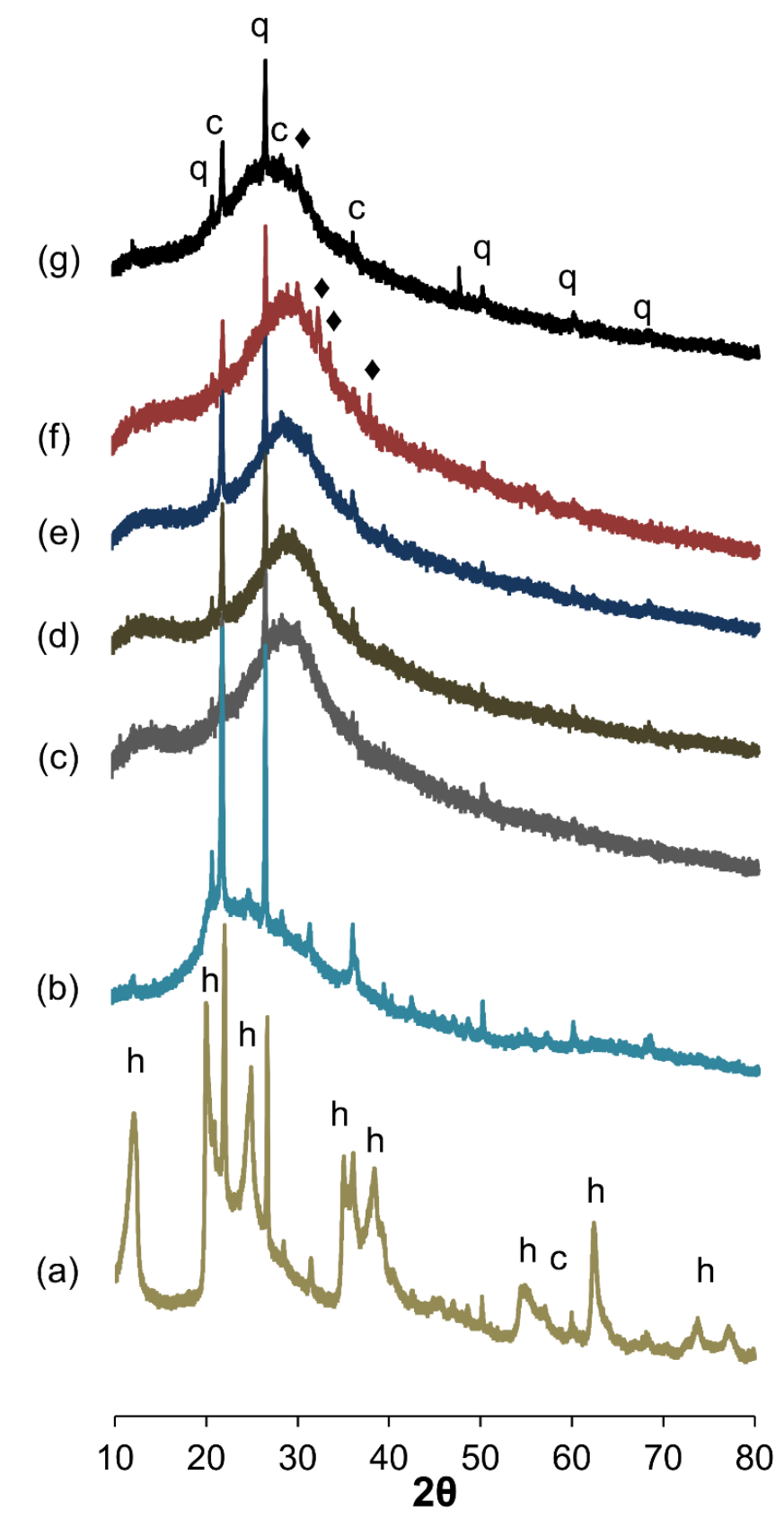

Figure 3.4 Fig. 1. XRD traces of the halloysite clay and the geopolymers. (a) halloysite clay, (b) dehydroxylated halloysite clay, (c) K-hiSi, (d) K-N, (e) Na-N, (f) Na-hiSi, (g) Na-hiSi-Seq. (h: aluminium silicate hydroxide (halloysite); q: quartz; c: cristobalite; $\mathrm{Na}_{2} \mathrm{CO}_{3} \cdot \mathrm{H}_{2} \mathrm{O}$ ) 
The XRD patterns of these geopolymers are unchanged by thermal treatment (Figure 3.5) which shows both $\mathrm{Na}$-hiSi and Na-hiSi-Seq after heating to $450{ }^{\circ} \mathrm{C}$ for $10 \mathrm{~min}$. The broadening of the amorphous background hump in the traces of both materials can be ascribed to the removal of some of the crystalline impurities.

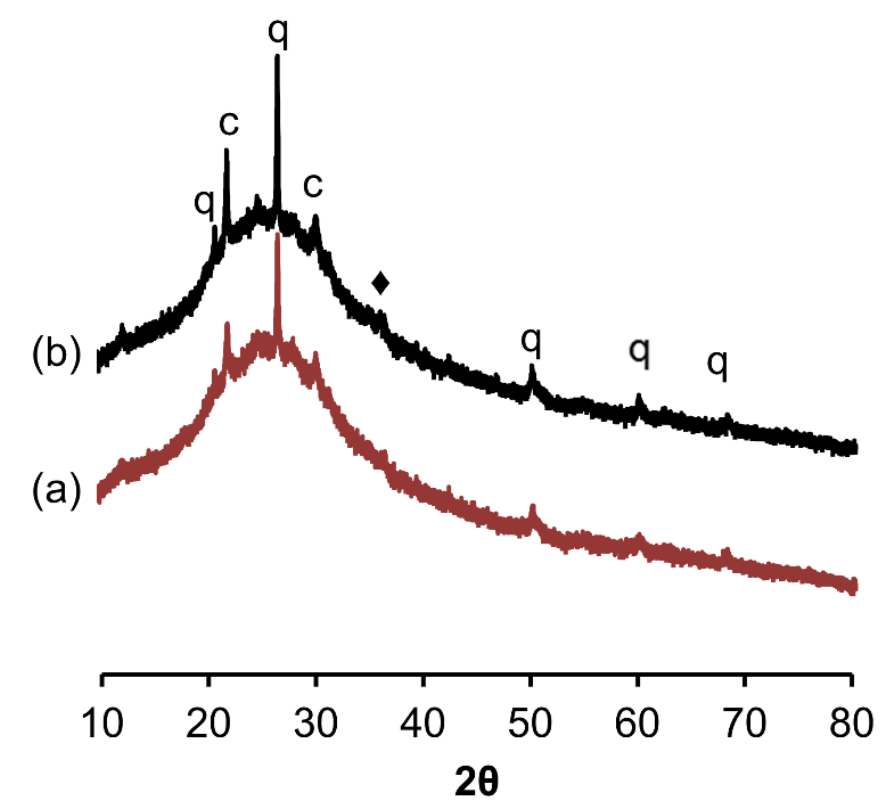

Figure 3.5 A representative XRD traces for one of the prepared geopolymers after heating to $450{ }^{\circ} \mathrm{C}$ for $10 \mathrm{~min}$. (a) Na-hiSi-450 ${ }^{\circ} \mathrm{C}$, (b) Na-hiSi-Seq-450 ${ }^{\circ} \mathrm{C}$. (q: quartz; c: cristobalite; $\diamond \mathrm{Na}_{2} \mathrm{CO}_{3} \cdot \mathrm{H}_{2} \mathrm{O}$ )

Solid-state ${ }^{27} \mathrm{Al}$ MAS NMR spectra of the geopolymer catalysts are shown in Figure 3.6. The chemical shifts of the Al spectra indicate the coordination number of the aluminium, which in well-formed geopolymers is almost entirely 4; this is seen to be the case in the spectra of all the geopolymers, in which the chemical shift of the principal resonance is typically tetrahedral $(\sim 60 \mathrm{ppm}){ }^{[69,91]}$. The slight shift to lower frequency in the "hiSi" geopolymers probably reflects their higher Si/Al ratio (Figure 3.6a and 3.6d). Dealumination is also confirmed in the sequentially post-synthetic treated geopolymer by a further shift to $57.8 \mathrm{ppm}$ (Figure 3.6e). Two additional small, broad ${ }^{27} \mathrm{Al}$ resonances at about $4 \mathrm{ppm}$ in the Na-geopolymer samples (Figure 3.6c and 3.6d) arise from octahedrally-coordinated $\mathrm{AlO}_{6}{ }^{[91]}$, indicating the presence of a small amount of unreacted clay mineral, in which the $\mathrm{Al}$ is in solely 6 -fold coordination. The absence of unreacted starting material in the K-based samples (Figure 3.6a and 3.6b) 
indicates more complete geopolymer formation in these samples, as expected from previous studies ${ }^{[69]}$.

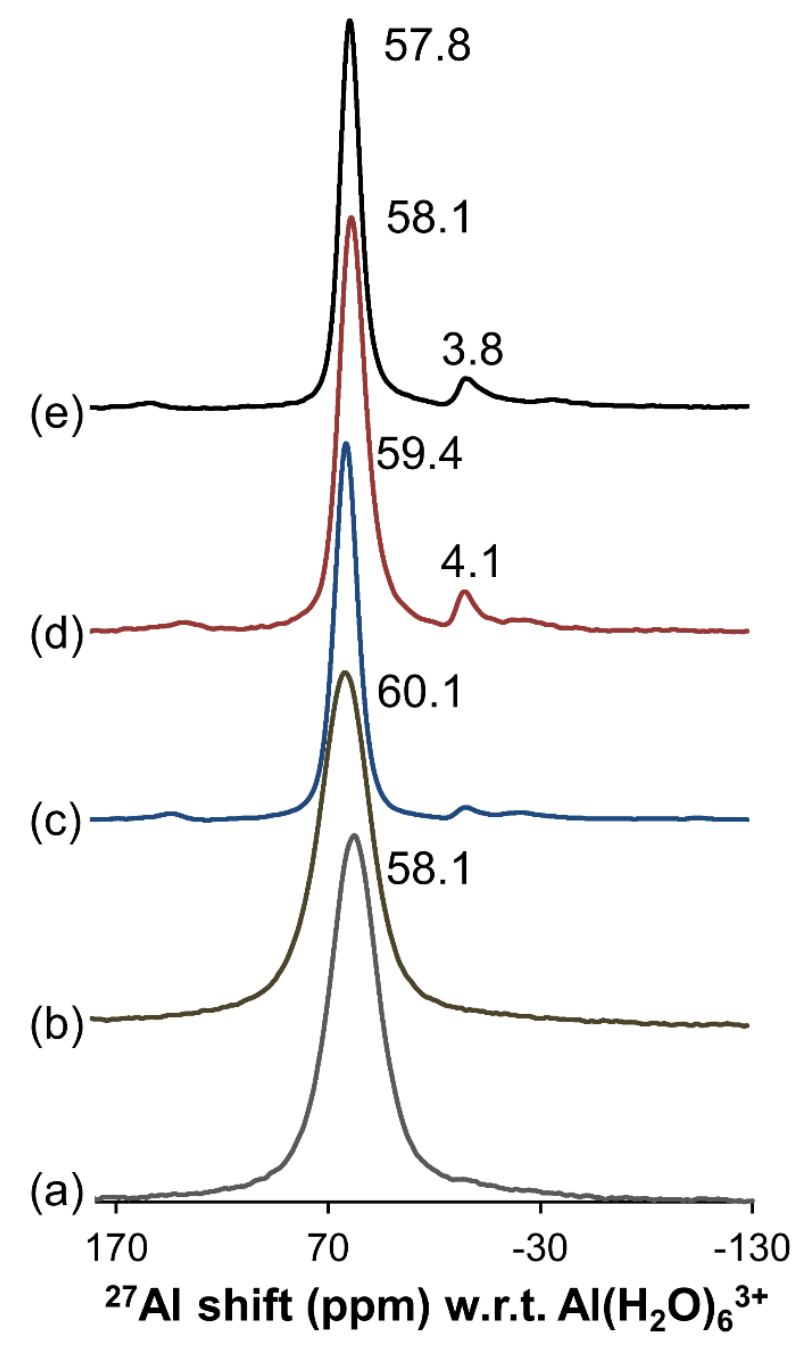

Figure 3.6 ${ }^{27} \mathrm{Al}$ MAS NMR spectra of the as-synthesised geopolymer catalysts. (a) KhiSi, (b) K-N, (c) Na-N, (d) Na-hiSi, (e) Na-hiSi-Seq.

The ${ }^{29}$ Si MAS NMR spectra of K-hiSi, K-N, and Na-N (Fig. 3.7) show a single broad resonance in the range 85-95 ppm, typical of a well-formed geopolymer containing tetrahedral Si coordinated to $4 \mathrm{Al}$ atoms ${ }^{[91]}$. The Na-hiSi sample (Fig. 3.7d) contains an additional resonance at about $-94.4 \mathrm{ppm}$, indicating the presence of $\mathrm{Si}\left(\mathrm{Q}^{3}\right)$ units in which the $\mathrm{Si}$ is surrounded by $3 \mathrm{Al}{ }^{[91]}$. The Na-hiSi-Seq sample (Figure 3.7e) also contains these two resonances, but shifted to lower frequencies due to dealumination. By contrast with the Na-hiSi spectrum, the intensity of the $\mathrm{Si} \mathrm{Q}^{3}$ resonance at $-97.3 \mathrm{ppm}$ in the hiSi-Seq sample is greater than that of $\mathrm{Si}^{4}$ at $-92.1 \mathrm{ppm}$, reflecting a higher concentration of silanol groups formed in the post-synthetic treatment. 


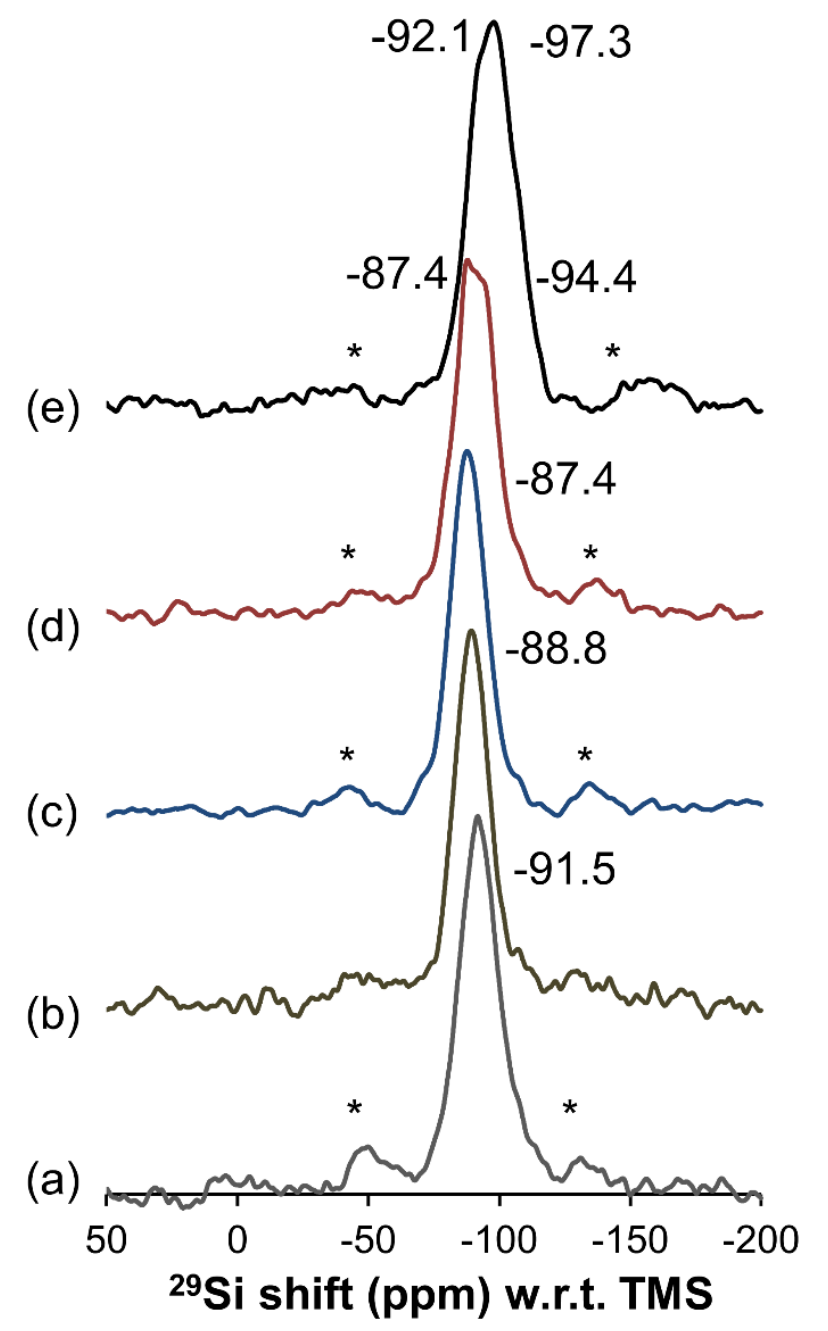

Figure 3.7 ${ }^{29} \mathrm{Si}$ MAS NMR spectra of the as-synthesised geopolymer catalysts. (a) KhiSi, (b) K-N, (c) Na-N, (d) Na-hiSi, (e) Na-hiSi-Seq. The asterisks denote spinning side bands.

A well-formed geopolymer structure was also confirmed by FTIR spectroscopy (Figure 3.8). All the parent geopolymers contain a typical strong broad peak at 1000 $\mathrm{cm}^{-1}$ ascribed to the Si-O-Al stretching vibration, with a shoulder at around $1080 \mathrm{~cm}^{-1}$ due to the Si-O-Si stretch and another shoulder at about 880 from the $\mathrm{Si}-\mathrm{OH}$ bending mode ${ }^{[137]}$. Another small peak at $1400 \mathrm{~cm}^{-1}$ arises from carbonate formed by atmospheric carbonation of the geopolymer to form $\mathrm{Na}_{2} \mathrm{CO}_{3}$, as detected by XRD (Figure 3.4). Carbonation is also confirmed by the broad $\mathrm{CO}_{2}$ asymmetric stretching vibration at about $2347 \mathrm{~cm}^{-1}{ }^{[138]}$. A broad peak at about $3500 \mathrm{~cm}^{-1}$ is assigned to hydrogen bonded silanol nests $\left(3500-3400 \mathrm{~cm}^{-1}\right){ }^{[99,124]}$ while the small peak at 1640 $\mathrm{cm}^{-1}$ is due to the $\mathrm{H}-\mathrm{OH}$ stretching mode from adsorbed water ${ }^{[139]}$. It is seen that the intensity of the silanol nests for the Na-hiSi (Figure 3.8d) is the highest of all the 
geopolymers. This is consistent with the ${ }^{29} \mathrm{Si}$ MAS NMR spectra in which the resonance at around $-94.4 \mathrm{ppm}$ assigned to $\mathrm{Si}\left(\mathrm{Q}^{3}\right)$ surrounded by $3 \mathrm{Al}$, indicating the presence of silanol groups.

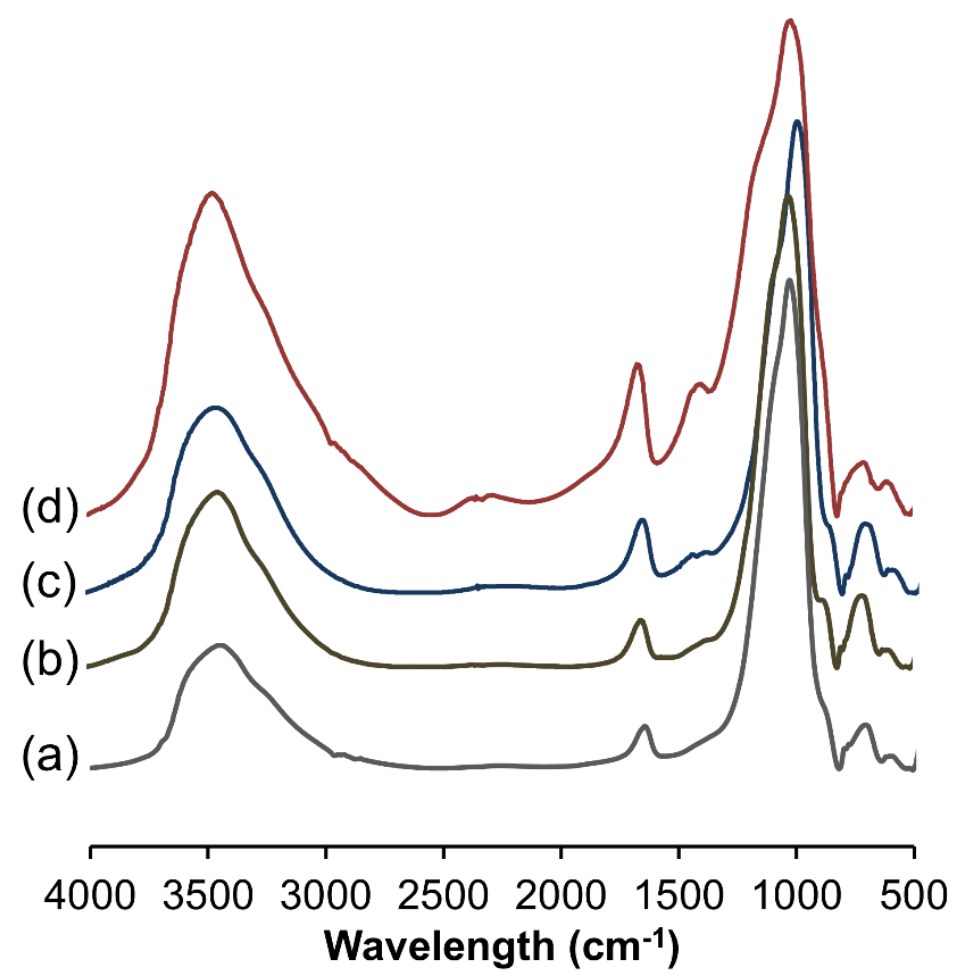

Figure 3.8 FTIR spectra of the parent geopolymers. (a) K-hiSi, (b) K-N, (c) Na-N, (d) Na-hiSi.

The SEM micrographs of the morphology of the synthesised geopolymer catalysts (Figure 3.9) show rounded particles with an average diameter of $~ 20$ $\mu \mathrm{m}$. At higher magnification the geopolymer particles are seen to be composed of small nanoparticles aggregated to form larger particles, creating voids of different widths within the particles.

The particle size distribution of selected geopolymers is shown in Figure 3.10. The average particle size of all the synthesised geopolymers is $\sim 20 \mu \mathrm{m}$, which is in agreement with the estimates from the SEM images. The sequential treatment slightly affects the particle size by shifting its average value to a slightly lower range, down to $11 \mu \mathrm{m}$ for the $\mathrm{K}-\mathrm{hiSi}$-Seq. 

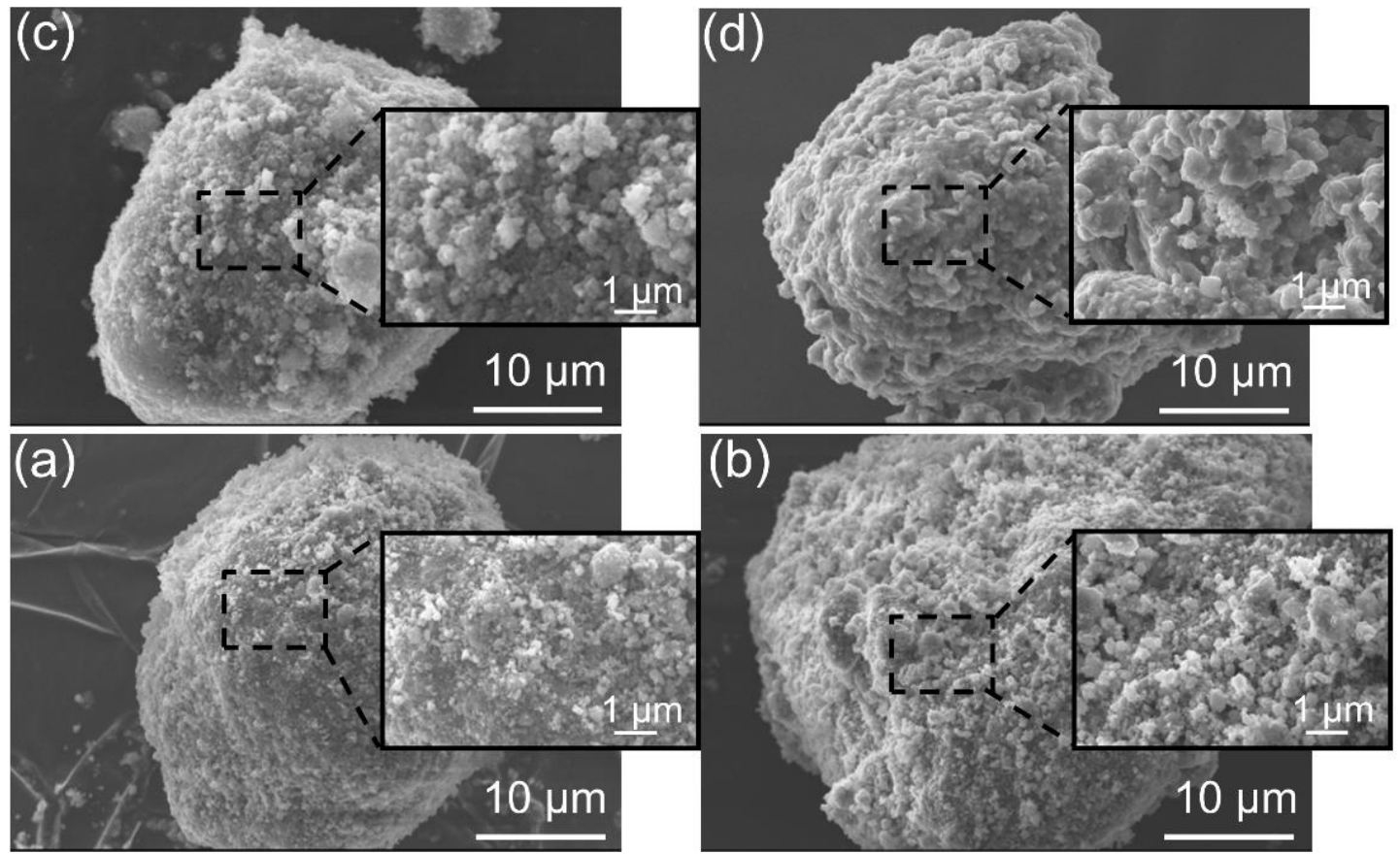

Figure 3.9 SEM micrographs of the prepared geopolymers. (a) K-hiSi, (b) K-N, (c) Na$\mathrm{N}$, (d) Na-hiSi.

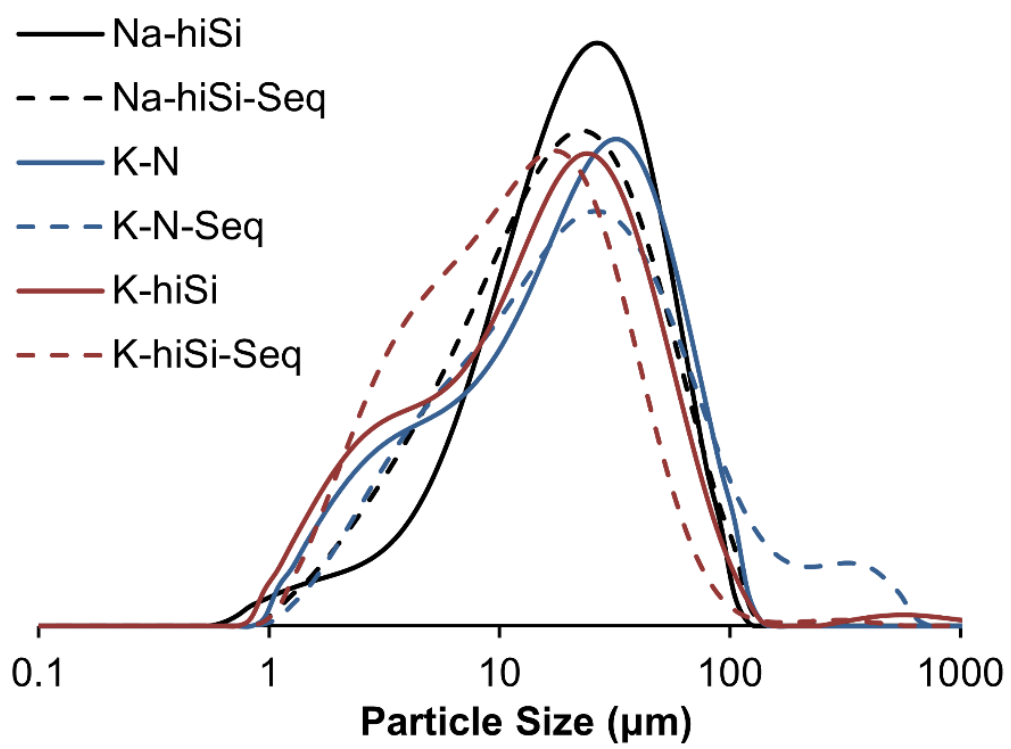

Figure 3.10 Particle size distribution of selected geopolymers before and after the sequential treatment.

The $\mathrm{N}_{2}$ adsorption-desorption isotherms of the parent and the sequentially treated geopolymer catalysts are shown in Figure 3.11 and the corresponding porosity values in Table 3.1. 
Of the synthesised geopolymers, only the Na-hiSi sample shows a type I isotherm typical of microporous materials, whereas all the other geopolymers show type IV isotherms with unique hysteresis loops indicating their mesoporosity. The hysteresis loop is of type $\mathrm{H} 3$ with unlimited gas uptake at high $p / p^{o}$, which is associated with aggregates possessing slit-like pores ${ }^{[103]}$. This is consistent with SEM micrographs (Figure 3.9) which indicate the presence of small nanoparticles aggregated to form larger particles, creating voids of different sizes within the particles.

Table 3.1 Textural and acidic properties of the various geopolymer-based catalysts.

\begin{tabular}{cccccc}
\hline Catalyst & $\begin{array}{c}\text { Acid content } \\
(\mathbf{m m o l} / \mathbf{g})^{\mathbf{a}}\end{array}$ & $\boldsymbol{S}_{\text {BET }}\left(\mathbf{m}^{2} / \mathbf{g}\right)$ & $\begin{array}{c}\boldsymbol{V}_{\text {total }} \\
\left(\mathbf{c m}^{\mathbf{3}} / \mathbf{g}\right)^{\mathbf{b}}\end{array}$ & $\begin{array}{c}\boldsymbol{V}_{\text {meso }} \\
\left(\mathbf{c m}^{\mathbf{3}} / \mathbf{g}\right)^{\mathbf{c}}\end{array}$ & $\boldsymbol{D}_{\text {pore }}(\mathbf{n m})^{\mathbf{c}}$ \\
\hline K-N & 0.27 & 50.0 & 0.20 & 0.20 & 22.1 \\
K-N-Seq & 0.33 & 64.7 & 0.33 & 0.32 & 28.1 \\
K-hiSi & 0.30 & 40.2 & 0.32 & 0.32 & 42.8 \\
K-hiSi-Seq & 0.34 & 59.6 & 0.39 & 0.39 & 50.2 \\
Na-N & 0.18 & 18.0 & 0.15 & 0.15 & 45.1 \\
Na-N-Seq & 0.24 & 42.2 & 0.25 & 0.24 & 47.1 \\
Na-hiSi & 0.38 & 135.0 & 0.11 & 0.01 & 2.7 \\
Na-hiSi-Seq & 0.43 & 211.5 & 0.28 & 0.26 & 16.4 \\
\hline
\end{tabular}

${ }^{a}$ total (determined from TGA profile of adsorbed pyridine)

${ }^{\mathrm{b}}$ single point at $p / p^{o}=0.995$

${ }^{c}$ measured by BJH (adsorption branch).

The pore size distribution of some of the synthesised geopolymers is in the range of $<10 \mathrm{~nm}$ to $100 \mathrm{~nm}$, indicating the presence of macroporosity which was also observed by TEM (Figure 3.12). However, the average pore size distribution of all the catalysts is in the range of $10-40 \mathrm{~nm}$. 

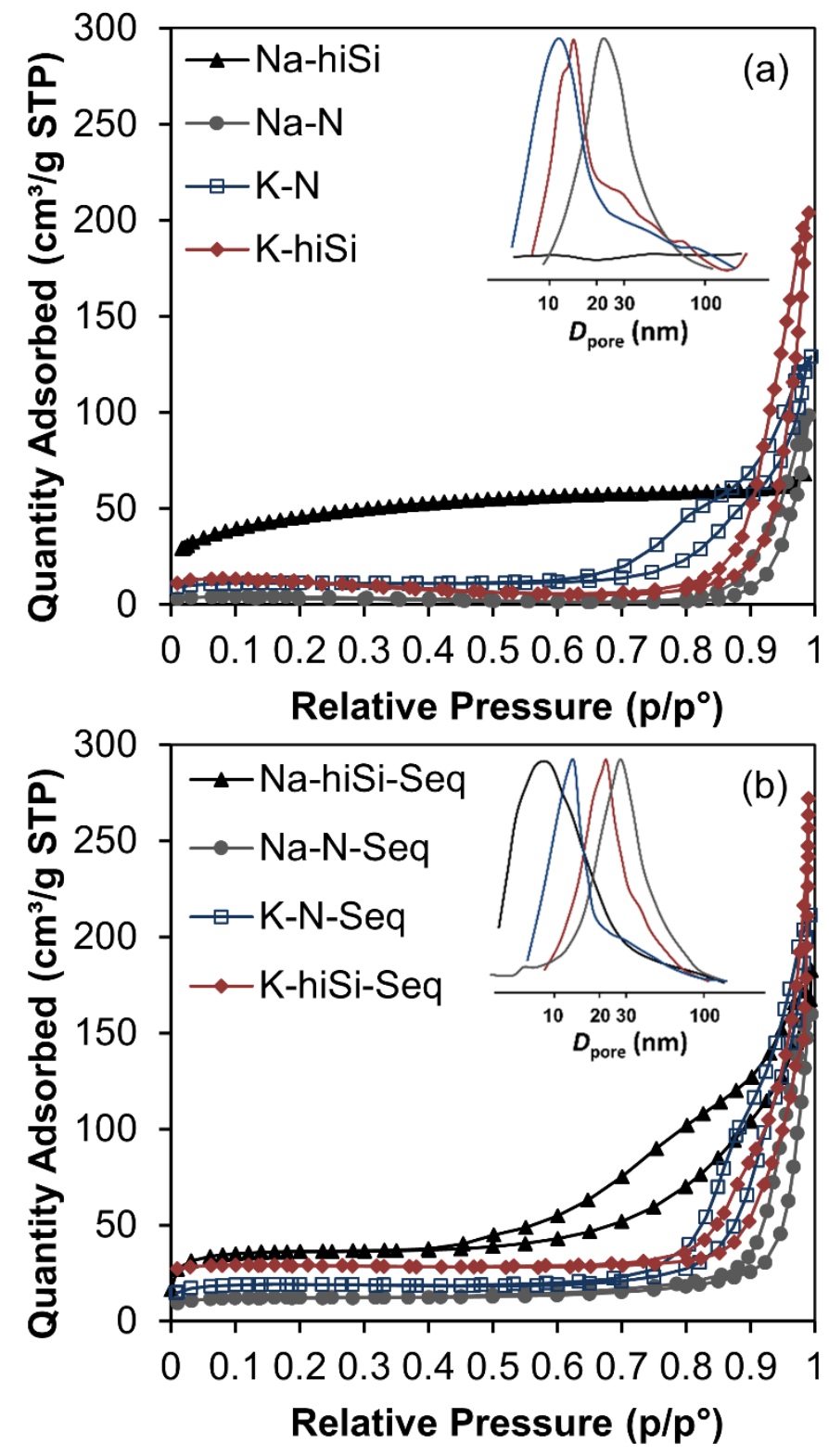

Figure 3.11 $\mathrm{N}_{2}$ adsorption-desorption isotherms of the geopolymer-based catalysts.

Around $80 \mathrm{wt}$. \% of the solid catalyst originally used was recovered after the sequential treatment and the impact of the treatment on the porosity and acidity of the catalyst was investigated. Figure $3.11 \mathrm{~b}$ shows the effect of the post-synthetic treatment on the porosity of the geopolymer catalysts. After the sequential treatment, the surface area, pore volume and pore width are almost doubled for some of the starting geopolymers (Table 3.1). Figure $3.11 \mathrm{~b}$ also shows that the sequential treatment on Na-hiSi geopolymer results in the formation of a hierarchical geopolymer (containing both micro and meso scale pores). 
The influence of these treatments on the porosity can also be seen in the TEM images of the geopolymer catalysts before and after the sequential treatment (Figure 3.12).

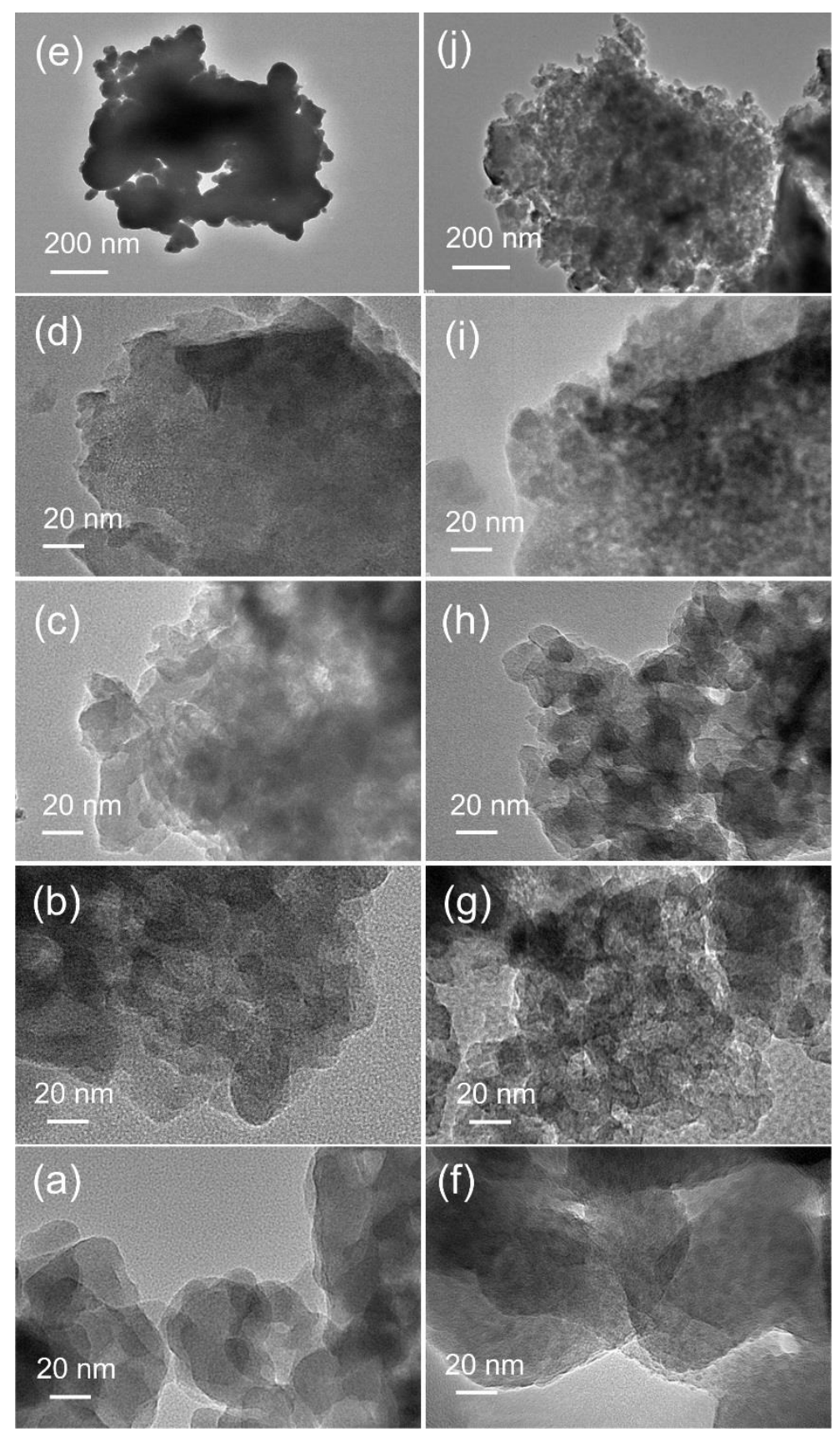

Figure 3.12 TEM micrographs of the parent geopolymers (a-d) and the sequentially treated geopolymers (e-h). (a) K-hiSi, (b) K-N, (c) Na-N, (d) and (e) Na-hiSi, (f) K-hiSi -Seq, (g) K-N-Seq, (h) Na-N-Seq, (i) and (j) Na-hiSi-Seq.

Geopolymers K-hiSi, K-N, and the Na-N geopolymers (Figure 3.12a-c) exhibit large mesopores in the form of voids contained within aggregates; this is consistent with the $\mathrm{N}_{2}$ adsorption-desorption isotherm of these samples which show an $\mathrm{H} 3$ type of 
hysteresis associated with particle aggregates, producing slit-like pores ${ }^{[103]}$. By contrast, Na-hiSi (Figure (3.12d) contains small randomly oriented non-uniform micropores. Figure 3.12f-i shows that additional new mesopores are introduced into all the catalysts after the sequential treatment, consistent with the results obtained from $\mathrm{N}_{2}$ isotherms and the corresponding data of Table 3.1. TEM micrographs at lower magnification for both Na-hiSi and Na-hiSi-Seq (Figure $3.12 \mathrm{e}$ and $\mathrm{j}$ respectively) show that interparticle voids were introduced, explaining the high surface area resulting from the post synthetic treatment for this particular catalyst.

\subsubsection{Generation of acid sites for Beckmann rearrangement}

The ion-exchange process was monitored by FTIR spectroscopy (Figure 3.13), which shows selected spectra of a sequence of Na-hiSi samples throughout the various stages of treatment to produce the catalyst.

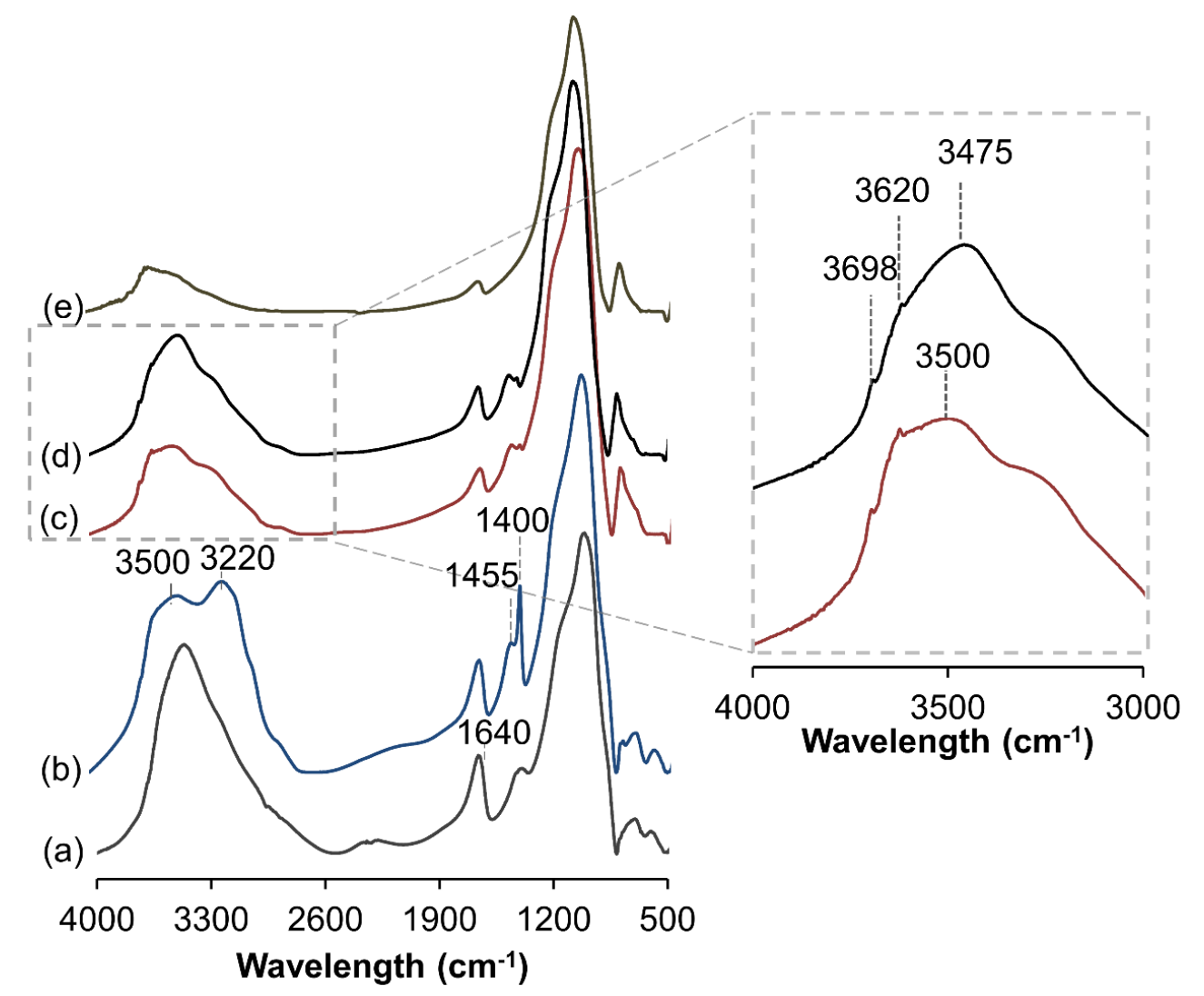

Figure 3.13 FTIR spectra of (a) as-prepared Na-hiSi, (b) $\mathrm{NH}_{4}{ }^{+}$-ion-exchanged Na-hiSi, (c) after thermal treatment at $450{ }^{\circ} \mathrm{C}$ for10 min, (d) Na-hiSi-Seq after thermal treatment at $450{ }^{\circ} \mathrm{C}$ for $10 \mathrm{~min}$, (e) Na-hiSi after thermal treatment at $550{ }^{\circ} \mathrm{C}$ for $10 \mathrm{~min}$. 
After ion-exchange with $\mathrm{NH}_{4}{ }^{+}$, additional bands appear (Figure 3.13b); one at about $3200 \mathrm{~cm}^{-1}$ is assigned to the N-H asymmetric stretching vibration, while two other bands at $1455 \mathrm{~cm}^{-1}$ and $1400 \mathrm{~cm}^{-1}$ are the typical bending modes of ammonium ions. Ion exchange is also accompanied by the disappearance of the $\mathrm{CO}_{2}$ asymmetric stretching band at $2347 \mathrm{~cm}^{-1}$. due to the removal of the Na cations. After heating to 450 ${ }^{\circ} \mathrm{C}$ for $10 \mathrm{~min}$. (Fig. 3.13c, d), the principal N-H stretching band begins to broaden and only traces remain of the $\mathrm{N}-\mathrm{H}$ bending modes, resulting from decomposition of the $\mathrm{NH}_{3}$. The bands at 3500 and $3475 \mathrm{~cm}^{-1}$ are also slightly broadened after thermal treatment due to destruction of the silanol nests. Two new peaks appearing at 3700 and $3620 \mathrm{~cm}^{-1}$ are attributed to H-bonded vicinal silanols and bridging hydroxyls (Bronsted $\mathrm{OH})$ respectively ${ }^{[99,140]}$. These weak acidic silanols together with minor amounts of Bronsted acid sites are ideal active sites for the Beckmann rearrangement, suggesting that these materials should act as efficient catalysts for this reaction. On the other hand, a total degradation of the $\mathrm{NH}_{4}{ }^{+}$occurs at $550{ }^{\circ} \mathrm{C}$, evidenced by the total disappearance of the $\mathrm{N}-\mathrm{H}$ bending and stretching bands, associated with severe destruction of the structural hydroxyls (Figure 3.13e).

Thermal treatment of aluminosilicates normally brings about dealumination, producing EFAl which usually acts as Lewis acid sites. Figure 3.14 shows the effect of thermal treatment at different temperatures on Na-hiSi, in which a higher proportion of EFAl species is generated by heating at $550{ }^{\circ} \mathrm{C}$. 


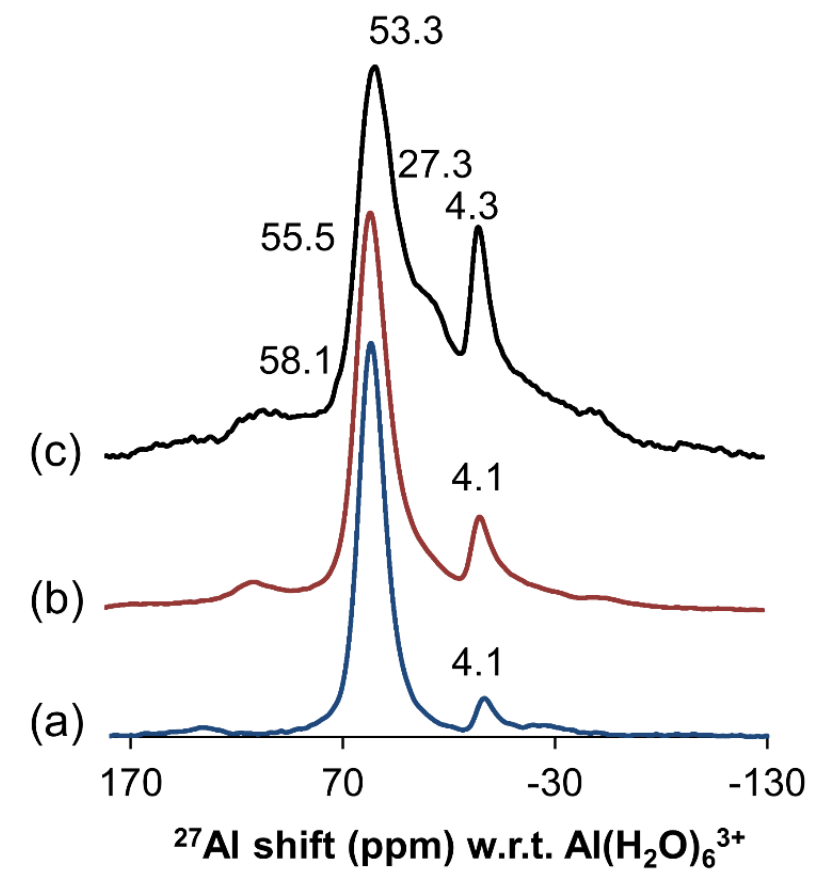

Figure 3.14 ${ }^{27} \mathrm{Al}$ MAS NMR of a selected geopolymer after thermal treatment at different temperature. (a) Na-hiSi as-synthesised, (b) Na-hiSi after thermal treatment at $450{ }^{\circ} \mathrm{C}$ for $10 \mathrm{~min}$, (c) Na-hiSi after thermal treatment at $550{ }^{\circ} \mathrm{C}$ for $10 \mathrm{~min}$.

In the Beckmann rearrangement of cyclohexanone oxime to $\varepsilon$-caprolactam, the presence of Lewis acid sites in the catalyst usually leads to the formation of other byproducts, mainly cyclohexanone ${ }^{[129 \mathrm{c}]}$. For this reason, the catalysts for use in this reaction were thermally treated at $450{ }^{\circ} \mathrm{C}$, the temperature at which a reasonable degree of $\mathrm{NH}_{4}{ }^{+}$degradation takes place, generating the various types of hydroxyl groups that are required for this particular reaction, and at the same time only generating a small proportion of EFAl.

Figure 3.15 shows representative FTIR spectra of Na-hiSi and Na-hiSi-Seq geopolymers activated at $450{ }^{\circ} \mathrm{C}$ containing pyridine. The band at $1543 \mathrm{~cm}^{-1}$ is assigned to Bronsted acid sites while those at $1448 \mathrm{~cm}^{-1}, 1600 \mathrm{~cm}^{-1}$ and $1620 \mathrm{~cm}^{-1}$ are assigned to Lewis acid sites. The peak at $1490 \mathrm{~cm}^{-1}$ is ascribed to a combination of both Lewis and Bronsted acid sites ${ }^{[100,126 b]}$. The low intensity of the peaks in Figure 3.14 is probably due to interference with the N-H stretching bands at $1400-1450 \mathrm{~cm}^{-1}$ by the remaining $\mathrm{NH}_{4}{ }^{+}$. 


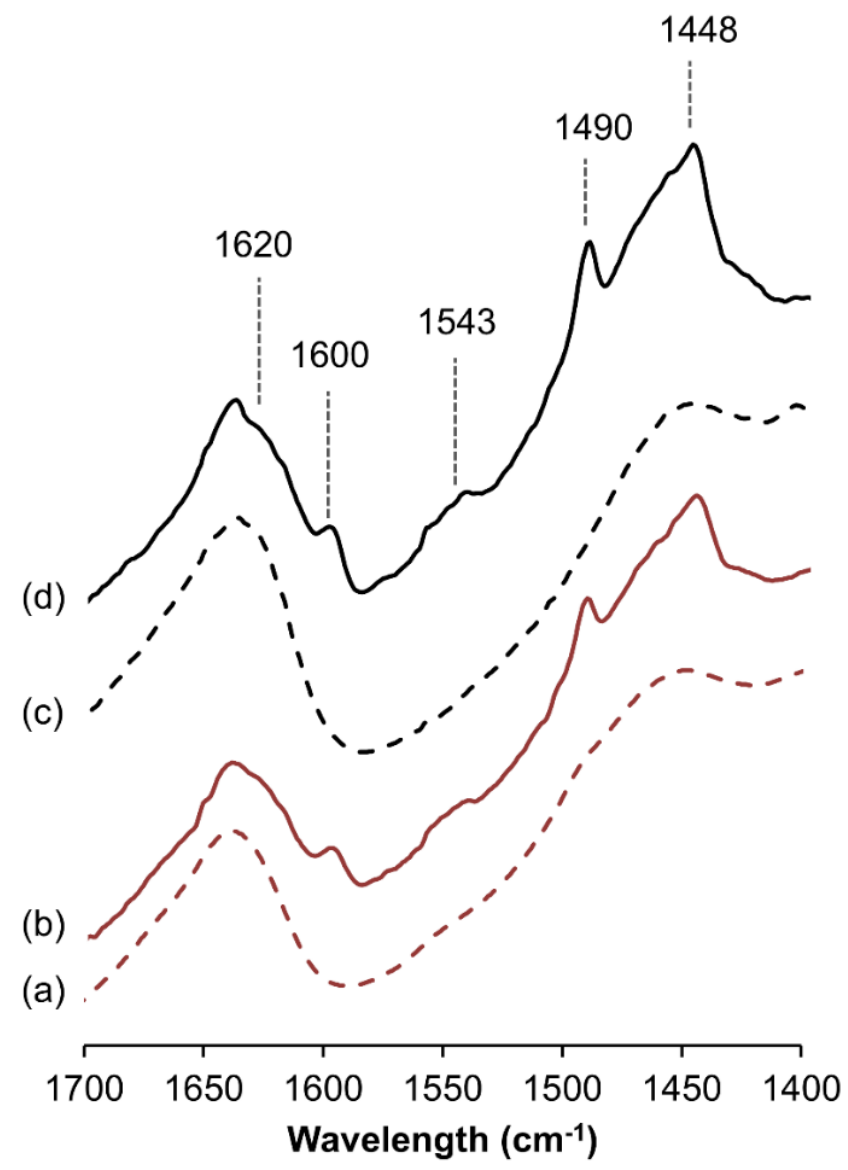

Figure 3.15 FTIR spectra of the activated geopolymer before and after adsorption of pyridine; (a) Na-hiSi without pyridine, (b) Na-hiSi-pyridine, (c) Na-hiSi-Seq without pyridine, (d) Na-hiSi-Seq-pyridine.

Figure 3.16 shows the TGA trace of pyridine desorption from selected catalysts, from which the total acidity was measured (and summarised in Table 3.1). The total acid amount measured for the catalysts with higher silicon content is higher than the total acidity of geopolymers with higher Al content, as shown in Table 3.1. This could be explained by analogy with zeolites in terms of the distribution of framework $\mathrm{Al}$ as described in section 1.3.1. 
The Na-hiSi-Seq sample (Figure 3.16b) shows a larger amount of pyridine desorbed in the higher temperature range $\left(160-350{ }^{\circ} \mathrm{C}\right)$, indicating a higher pyridine concentration and stronger acid sites in this catalyst compared to Na-hiSi (Figure 3.16a). The higher acidity of Na-hiSi-Seq (Table 3.1) can be ascribed to the higher proportion of EFAl species generated during the thermal pre-treatment. This suggestion is supported by ${ }^{27} \mathrm{Al}$ MAS NMR spectroscopy of both catalysts after thermal treatment at $450{ }^{\circ} \mathrm{C}$ for $10 \mathrm{~min}$ (Fig. 3.17).

(a)

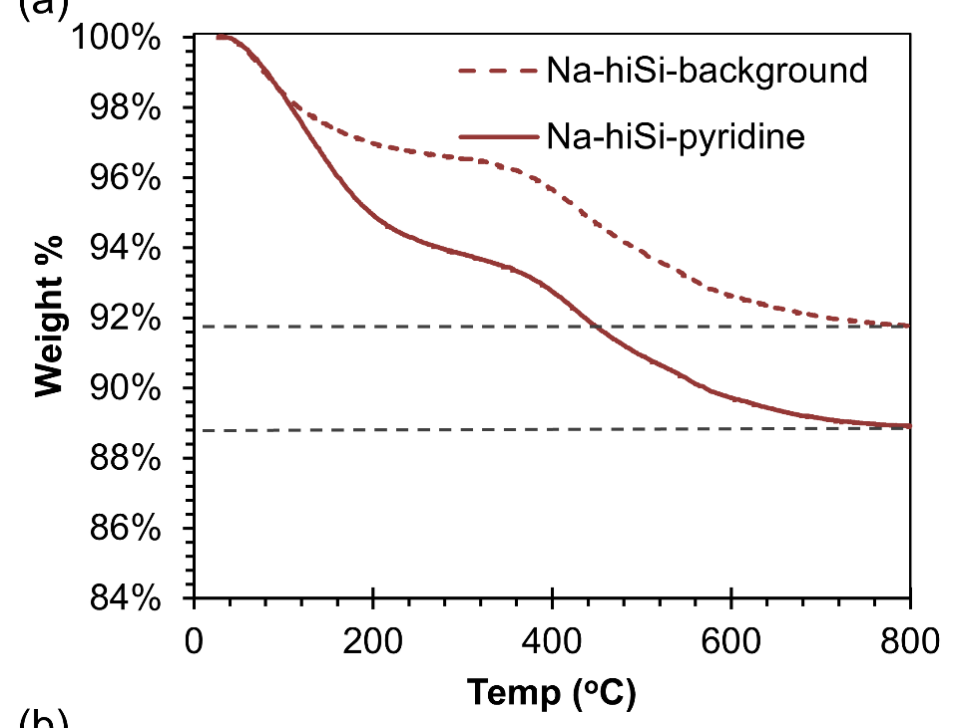

(b)

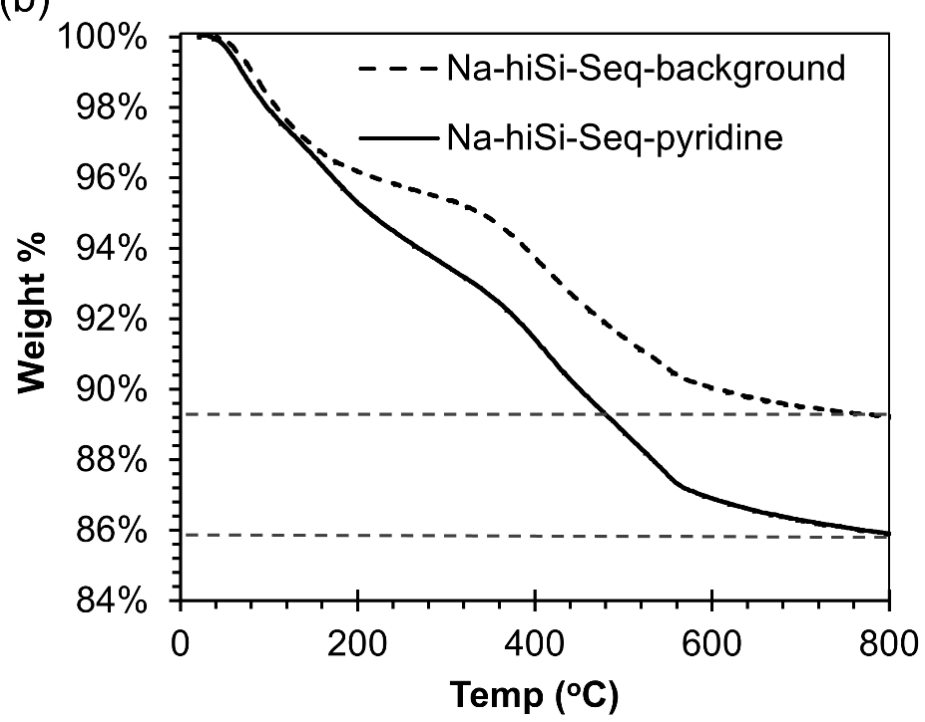

Figure 3.16 TGA traces of pyridine desorption. (a) sample Na-hiSi, (b) Na-hiSi-Seq. 


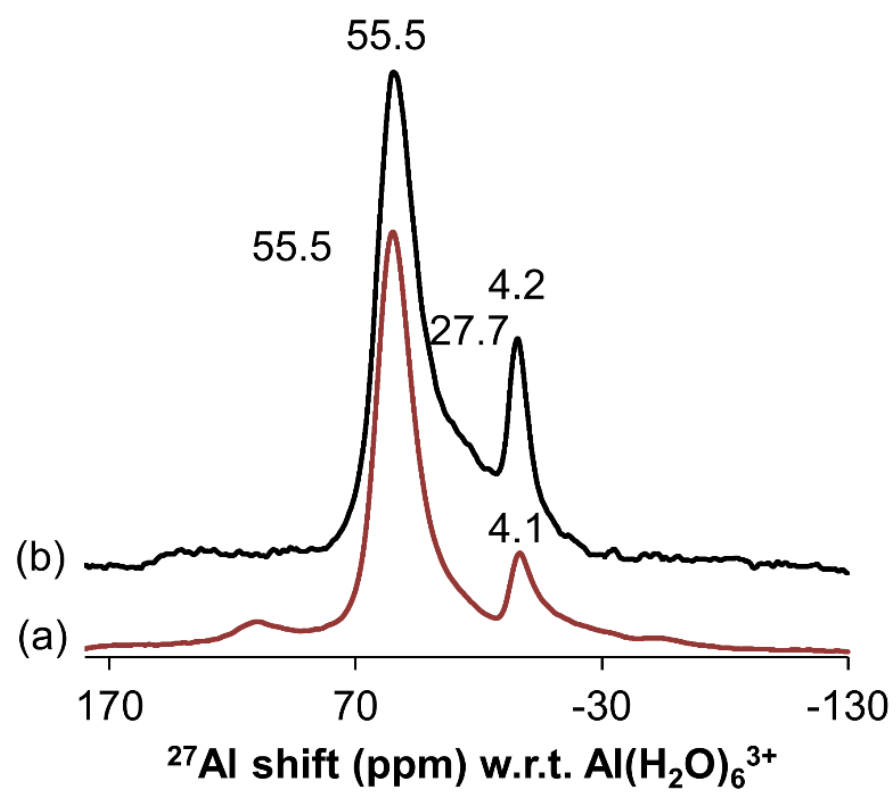

Figure 3.17 ${ }^{27} \mathrm{Al}$ MAS NMR spectra of Na-hiSi (a) and Na-hiSi-Seq (b) both heated to $450{ }^{\circ} \mathrm{C}$ for $10 \mathrm{~min}$.

The spectra indicate that the thermal treatment has produced a higher concentration of octahedrally-coordinated $\mathrm{Al}$ (the resonance at $4.2 \mathrm{ppm}$ ) and a more pronounced shoulder at $27.7 \mathrm{ppm}$ arising from 5-coordinated $\mathrm{Al}$ in the sequentially-treated solid compared to the as-synthesised geopolymer.

\subsubsection{Catalytic reactivity}

No conversion to the oxime occurred when a control sample was tested using the $\mathrm{NH}_{4}{ }^{+}$-geopolymer without thermal treatment. Moreover, catalysts that were thermally treated above $450{ }^{\circ} \mathrm{C}\left(500\right.$ and $\left.550{ }^{\circ} \mathrm{C}\right)$ gave almost $100 \%$ conversion of cyclohexanone oxime, but with $0 \%$ selectivity to caprolactam. As discussed above (section 3.3.2), this is ascribed to the EFAl species which act as Lewis acid sites and facilitate side reactions such as polymerisation, as well as causing hydrolysis to the oxime in presence of atmospheric $\mathrm{H}_{2} \mathrm{O}$ resulting in the formation of cyclohexanone. Since the results obtained from experiments carried out using a Schlenk line did not show any difference in the catalytic performance, the bulk of the experiments were carried out under the conditions described in section 3.2.2. 
A number of other solvents with different basicities and dielectric constants were used, as shown in table 3.2. However, apart from $\mathrm{PhCN}$, none of the other solvents used in conjunction with the geopolymer catalyst showed any activity. This result is ascribed the lower basicity of PhCN compared with DMSO and acetonitrile which results in reasonable adsorption on the catalyst surface, thereby facilitating the adsorption of the reactant and desorption of the product, and at the same time possessing the good proton affinity necessary for the reaction mechanism (see section 3.1). In addition, $\mathrm{PhCN}$ is adsorbed on the Lewis acidic sites and reacts with water forming benzamide, thus improving the selectivity to caprolactam ${ }^{[130 \mathrm{a}]}$.

Table 3.2 Different solvents used in Beckmann rearrangement reaction

\begin{tabular}{cccc}
\hline Solvent & Dielectric constant $^{\mathbf{a}}$ & Basicity $^{\mathbf{b}}$ & Boiling point $^{\left({ }^{\circ} \mathbf{C}\right)}$ \\
\hline $\begin{array}{c}\text { Dimethylsulfoxide } \\
\text { (DMSO) }\end{array}$ & 47.24 & 29.8 & 189.0 \\
$\begin{array}{c}\text { Acetonitrile } \\
\text { Benzonitrile } \\
(\text { PhCN) }\end{array}$ & 36.64 & 14.1 & 82.0 \\
Toluene & 25.20 & 11.9 & 190.7 \\
\hline
\end{tabular}

${ }^{a}$ values obtained from refs. ${ }^{[129 a, 130 a]}$

${ }^{\mathrm{b}}$ donor number $(\mathrm{kcal} / \mathrm{mol})^{[141]}$.

The catalytic performance of all the geopolymer-based catalysts for the rearrangement of cyclohexanone oxime to $\varepsilon$-caprolactam is illustrated in Figure 3.18 and the data, including the catalytic reproducibilities are shown in Table 3.3. Those geopolymer catalysts with low surface areas and large pore diameters (K$\mathrm{N}, \mathrm{K}-$ hiSi and Na-N, see Table 3.1) gave the highest conversion of oxime but very low selectivity for $\varepsilon$-caprolactam.

The principal by-product resulting from the use of these catalysts was cyclohexanone, due to hydrolysis of the oxime on the Lewis acid sites in the presence of atmospheric water. Benzamide was also formed as a hydrolysis product of the solvent $(\mathrm{PhCN})$ in a reaction which also requires Lewis acid sites, as discussed above. Traces of other by-products such as of 9-octadecenamide 
were also detected, resulting from polymerisation side reactions facilitated by the presence of different EFAl species generated during thermal pre-treatment.
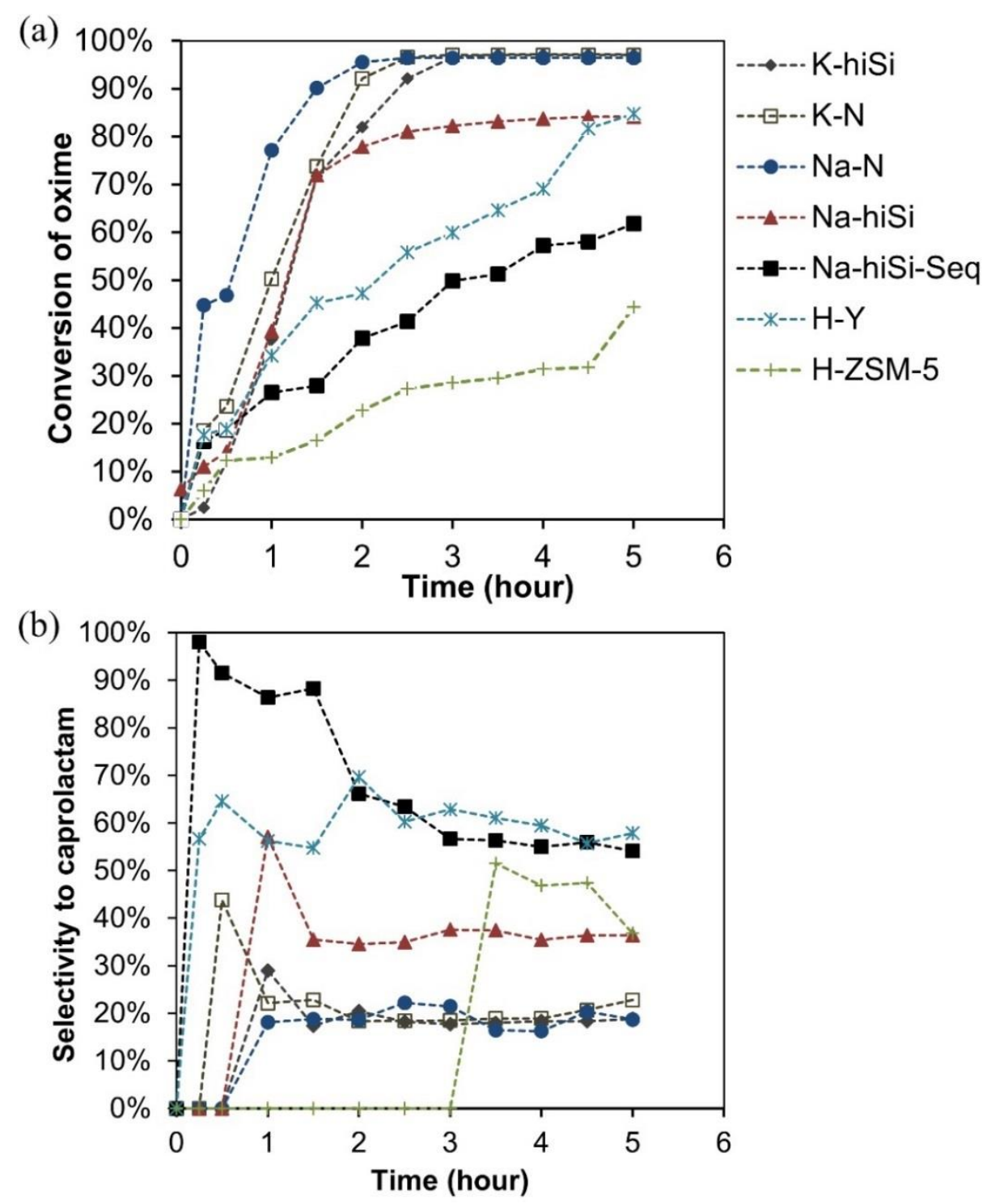

Figure 3.18 Catalytic activity of the various geopolymer-based catalysts compared with zeolite catalysts in the Beckmann rearrangement of cyclohexanone oxime to $\varepsilon$ caprolactam. (a) degree of conversion of the oxime, (b) selectivity for the caprolactam product. Reaction conditions: solvent $20 \mathrm{ml} \mathrm{PhCN;} 0.1$ g catalyst; $0.1 \mathrm{~g}$ oxime; $\mathrm{T}=130$ ${ }^{\circ} \mathrm{C}$.

A possible explanation for these results is that when the surface area is too low, the distribution of the active sites may be very limited, and rather than being adsorbed on the surface, the oxime will penetrate into the pores where active sites such as 5- and 6-coordinated EFAl are present, leading to the formation of other 
by-products. Thus, although the Na-hiSi catalysts with higher surface areas gave slightly lower conversion of oxime compared to the low surface area geopolymer-based catalysts, they showed higher selectivity towards $\varepsilon$ caprolactam. These results are in contrast to the behaviour of zeolite H-Y, which despite its high surface area, demonstrates a high degree of conversion that can be ascribed to its stronger surface acidity.

The influence of the post-synthetic treatment on the textural properties of the geopolymer are clearly seen; where the surface area was significantly increased (Table 3.1), this affected the selectivity towards $\varepsilon$-caprolactam. As discussed previously, silanol nests and weakly acidic vicinal silanols are the most likely active sites for this rearrangement. These sites are present in both the Na-hiSiSeq and Na-hiSi catalysts (Figure 3.13); the higher catalytic activity of the former compared to Na-hiSi can be attributed to the higher surface area and hence better distribution of these active sites in the former. These factors facilitate adsorption of the oxime molecules on the catalyst, thereby assisting the rearrangement reaction.

The performance of Na-hiSi-Seq was evaluated over a longer time period (Figure $3.19)$ in which the gradual increase in conversion and selectivity suggests that saturation of the active sites on the catalyst surface is occurring. If this is the case, it is possible that the problem could be overcome by running the reaction in a continuous flow system to remove the product as it is formed, making available further active sites on which the reaction can occur. 


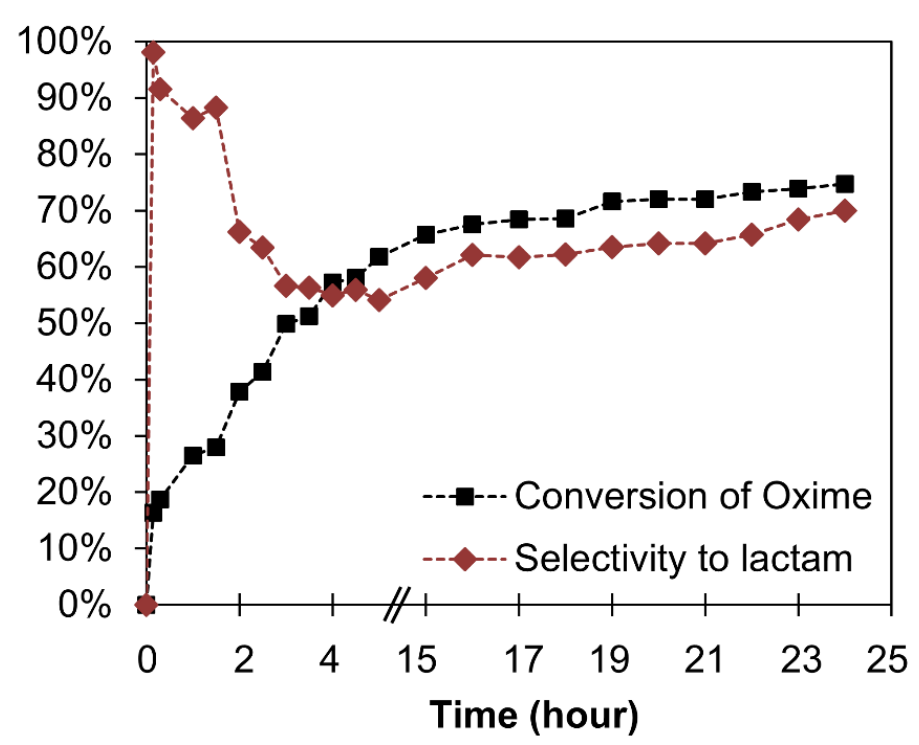

Figure 3.19 Catalytic performance of Na-hiSi-Seq as a function of reaction time. Reaction conditions: solvent $20 \mathrm{ml} \mathrm{PhCN}$; $0.1 \mathrm{~g}$ catalyst; $0.1 \mathrm{~g}$ oxime; $\mathrm{T}=130{ }^{\circ} \mathrm{C}$.

The effect of the reaction temperature and the substrate weight ratio are shown in Figure 3.18. Oxime conversion increased significantly from $\sim 33 \%$ at $90{ }^{\circ} \mathrm{C}$ to $\sim 87 \%$ at $140{ }^{\circ} \mathrm{C}$ (Figure 3.20a). These results are in agreement with the literature and can be attributed to the endothermic nature of the Beckmann rearrangement [126a, 142]. On the other hand, the selectivity to caprolactam is gradually reduced, which is expected since higher temperatures facilitate side reactions leading to the formation of other by-products. 
(a)

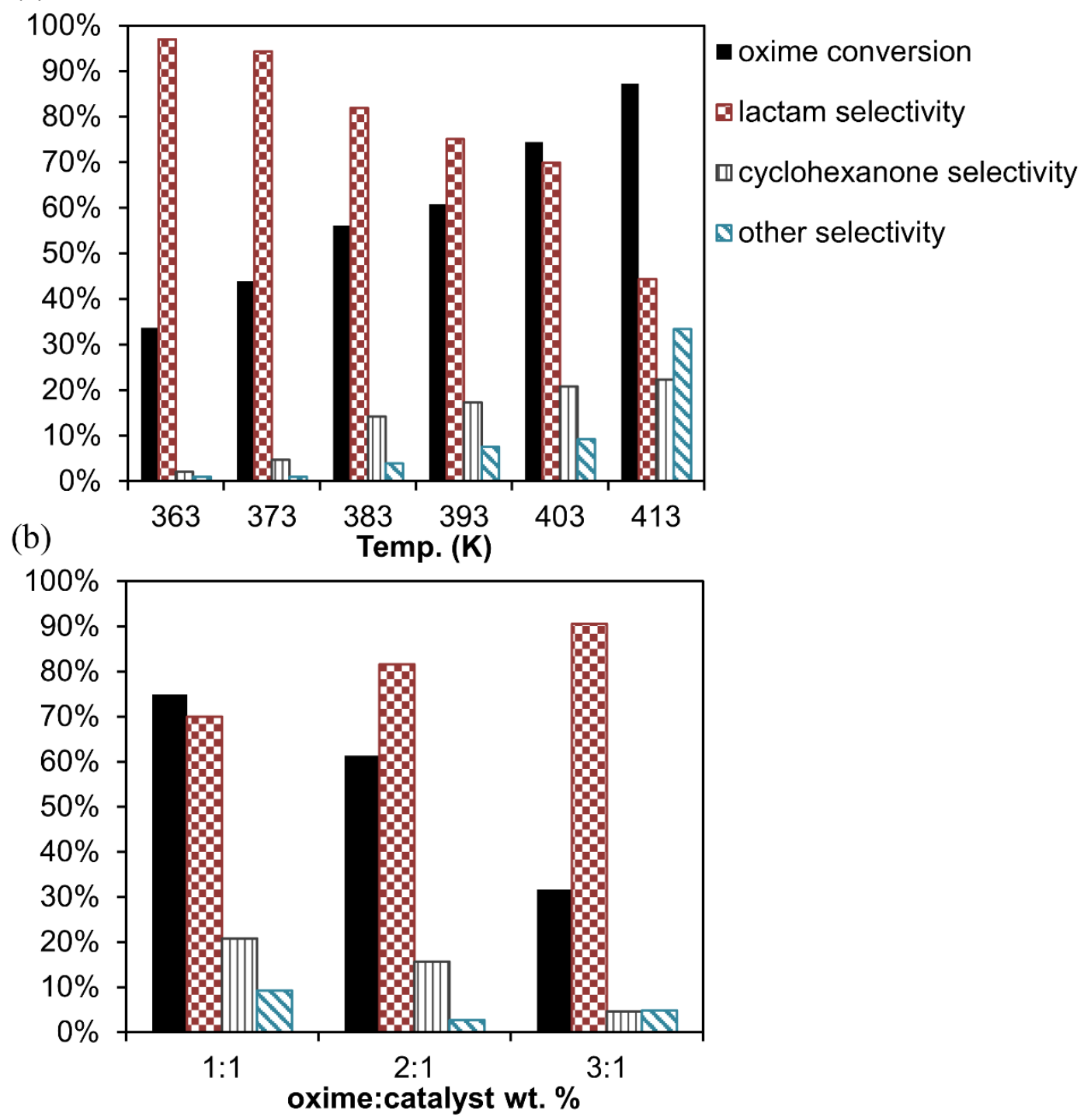

Figure 3.20 The influence of the reaction temperature (a), and the oxime: catalyst wt. $\%$ (b) on the Beckmann rearrangement reaction. The catalyst is Na-hiSi-Seq, solvent $\mathrm{PhCN}$.

With respect to the influence of the oxime: catalyst weight ratio, Figure $3.20 \mathrm{~b}$ shows that the conversion of the oxime is decreased by reducing the amount of the catalyst. The selectivity to the lactam, however, increases slightly as the amount of by-products formed decreases. These results can be attributed to the lower number of active sites responsible for both the rearrangement and production of the by-products. These results are in agreement with those reported for similar experiments with H-USY zeolite ${ }^{[129 c]}$. 
The reusability of Na-hiSi-Seq catalysts was studied for up to five reaction cycles and is shown in Figure 3.21. As expected, no significant change was observed in the yield of $\varepsilon$-caprolactam; since the active sites are generated within the geopolymer structure, these catalysts do not suffer the drawback of supported catalysts from which the active sites tend to be removed by leaching, deactivating the catalyst and adversely affecting its reusability.

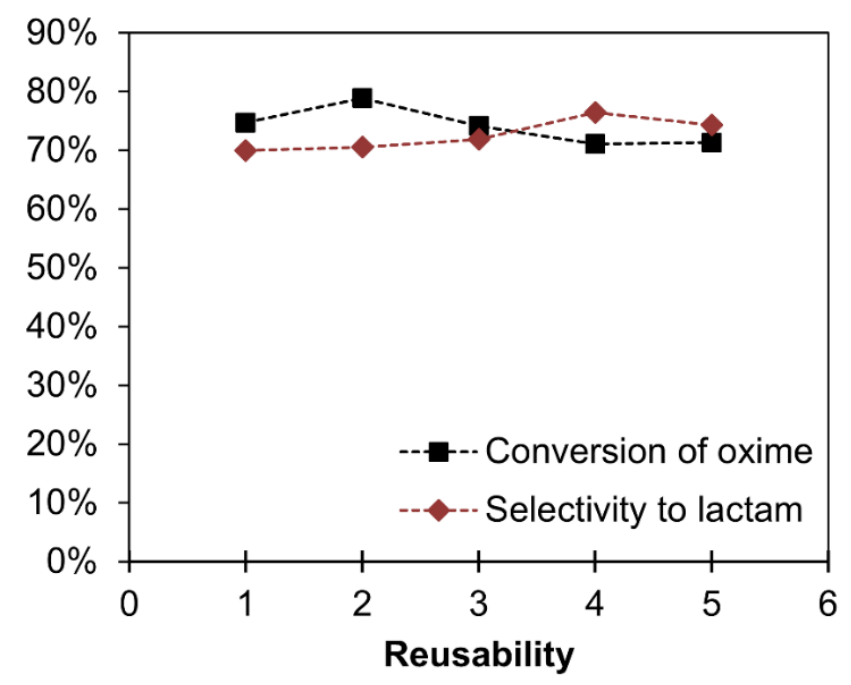

Figure 3.21 Reusability of Na-hiSi-Seq over five reaction cycles. Reaction conditions; 1:1:200 oxime: catalyst: solvent wt. $\% ; \mathrm{T}=130{ }^{\circ} \mathrm{C} ; \mathrm{t}=24 \mathrm{hr}$.

The structural stability of the used catalyst, after five recycles, was evaluated by XRD (Figure 3.22) which shows that there is little or no change in the spent geopolymer structure, illustrating the stability of the geopolymer-based catalysts. 


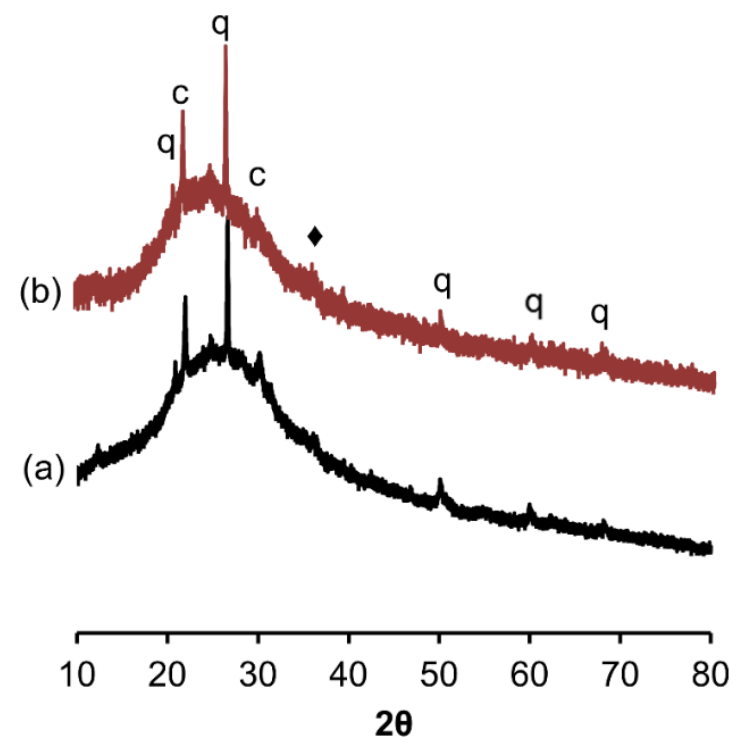

Figure 3.22 XRD traces of (a) fresh Na-hiSi-Seq catalyst and (b) the recovered catalyst after five reaction cycles.

The stability of the recovered catalyst was also examined by FTIR (Figure 3.23), showing the comparison between a fresh catalyst (Figure 3.23a) and the recovered one before and after the thermal treatment (Figure $3.23 \mathrm{~b}$ and $3.23 \mathrm{c}$ respectively).

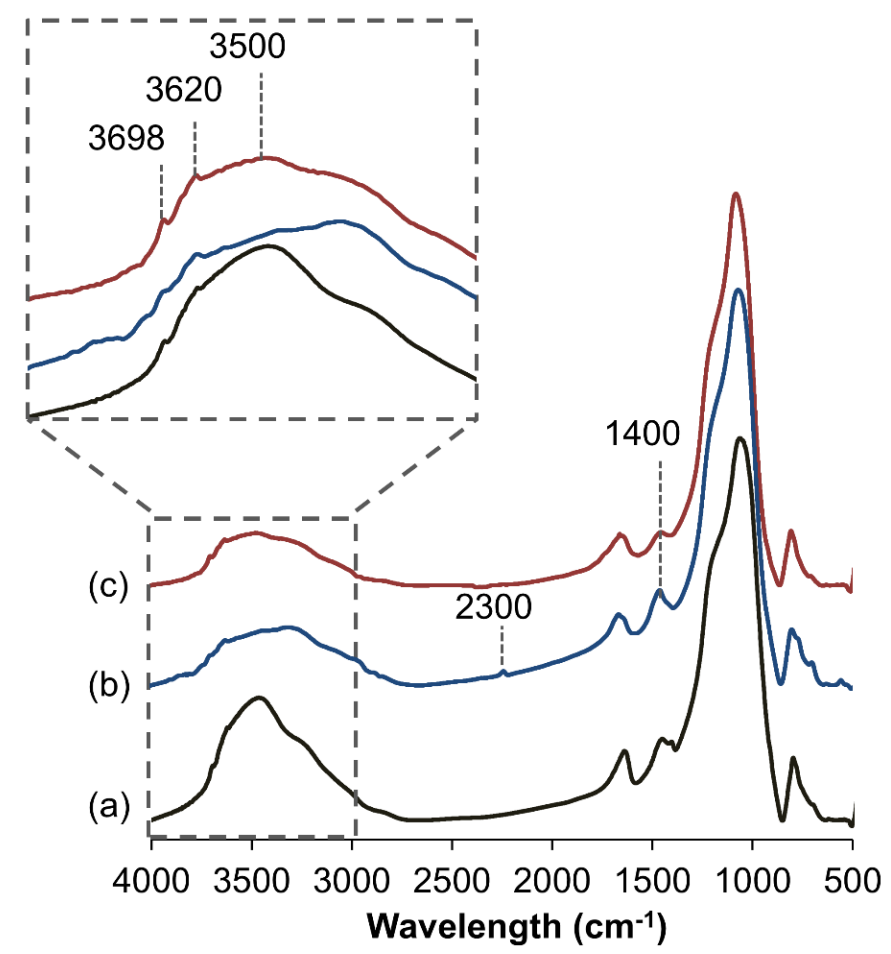

Figure 3.23 FTIR spectra of (a) fresh Na-hiSi-Seq catalyst, (b) the recovered catalyst after five reaction cycles, and (c) the recovered catalyst after heating to $450{ }^{\circ} \mathrm{C}$. 
The recovered catalyst (Figure 3.23b) contains small peaks at $\sim 1400$ and $\sim 2300 \mathrm{~cm}^{-1}$ arising from carbonation of the catalyst surface due to adsorbed species remaining after washing with acetone. These peaks disappeared after reactivating the catalyst at $450{ }^{\circ} \mathrm{C}$ which removed the adsorbed species (Figure 3.23c).

The performance of the new geopolymer-based catalysts was compared with other commonly used solid catalysts that have been previously reported for the liquid phase Beckmann rearrangement of cyclohexanone oxime. For the most meaningful possible comparison, only those studies are considered in which the reaction conditions were identical to the present work. These are shown in Table 3.3. The yield of caprolactam obtained by Na-hiSi-Seq is superior to the H-ZSM5 and H-Y zeolites tested in this study. The catalytic kinetics of Na-hiSi and NahiSi-Seq compared to HZSM-5 and H-Y zeolites are shown is Table 3.4. The obtained TON of both Na-hiSi and Na-hiSi-Seq catalysts are higher than those of the zeolites, indicating that the geopolymer catalysts are more efficient in the liquid phase Beckmann rearrangement reaction.

Table 3.3 Comparison of the catalytic performance of geopolymer-based catalysts with other catalysts for the liquid phase Beckmann rearrangement of cyclohexanone oxime to $\varepsilon$-caprolactam. Reaction conditions: solvent $20 \mathrm{ml} \mathrm{PhCN} ; 0.1 \mathrm{~g}$ catalyst; $0.1 \mathrm{~g}$ oxime; $\mathrm{T}=130{ }^{\circ} \mathrm{C} ; \mathrm{t}=5 \mathrm{hr}$.

\begin{tabular}{ccccc}
\hline \multirow{2}{*}{ Catalyst } & $\begin{array}{c}\text { Conversion } \\
\text { \% }\end{array}$ & \multicolumn{2}{c}{ Selectivity \% } & $\begin{array}{c}\text { Mass } \\
\text { balance } \\
\text { \% }\end{array}$ \\
\cline { 3 - 5 } Na-hiSi & $84 \pm 5$ & $36 \pm 3$ & $61 \pm 2$ & 98 \\
Na-hiSi-Seq $^{\#}$ & $75 \pm 4$ & $70 \pm 2$ & $21 \pm 1$ & 98 \\
H-Y zeolite $^{*}$ & $85 \pm 3$ & $58 \pm 1$ & $40 \pm 0.1$ & 98 \\
H-ZSM-5 $^{*}$ & $44 \pm 5$ & $37 \pm 3$ & $59 \pm 4$ & 98 \\
WOxZrO $_{2}{ }^{[128 a]}$ & 49 & 79 & & \\
Al-MCM-41 $^{[130 a]}$ & 51 & 89 & & \\
H-USY $^{[129 c]}$ & 98 & 75 & & \\
\hline
\end{tabular}

\footnotetext{
\# Reaction continued for 24 hours

* see section 3.2.1 for the catalysts preparation
} 
In addition, the catalytic activities of the geopolymer-based catalysts are superior to those reported for a wider range of solid catalysts which were tested in liquid phase systems, but under different reaction conditions. These other catalysts include zeolites (large pore $\mathrm{Y}$ and beta type ${ }^{[128 \mathrm{c}, 129 \mathrm{a}]}$ ), molecular sieves $(\mathrm{Nb}-$ MCM-41 ${ }^{[121]}$ and SBA-15-ar- $\left.\mathrm{SO}_{3} \mathrm{H}^{[130 \mathrm{~b}]}\right)$, functionalised silica $\left(\mathrm{H}_{2} \mathrm{SO}_{4} / \mathrm{SiO}_{2}\right.$ [131b] and $\mathrm{H}_{2} \mathrm{SO}_{4} / \mathrm{M} / \mathrm{SiO}_{2}{ }^{\text {[131c] }}$ ), HPAs such as Cs-phosphotungstic acid [128b], $\mathrm{Fe} / \mathrm{Cu}-1,3,5$-benzenetricarboxylate (MOFs) ${ }^{[128 c]}$ and ionic liquids (cyanuric chloride in imidazolium-based ILs ${ }^{[132 \mathrm{a}]}$, [Bis-BsImD] [OTf $\left.]_{2}-\mathrm{ZnCl}_{2}\right]^{[132 \mathrm{~b}]}$ and $\left.\left[\mathrm{C}_{3} \mathrm{SO}_{3} \mathrm{Hmim}\right][\mathrm{Cl}]-\left[\mathrm{ZnCl}_{2}\right]^{[132 \mathrm{c}]}\right)$.

Table 3.4 Reaction kinetics of Beckmann rearrangement of cyclohexanone oxime measured at $5 \mathrm{hr}$. reaction time over selected geopolymers and zeolites.

\begin{tabular}{cccc}
\hline Catalyst & $\begin{array}{c}\text { Specific reaction rate } \\
\left(\mathbf{x 1 0 0 ~} \mathbf{~ m m o l} \text { lactam. } \mathbf{g ~ c a t ~}^{\mathbf{- 1}} \cdot \mathbf{h}^{\mathbf{- 1}}\right)\end{array}$ & TON & TOF $\left(\mathbf{h}^{\mathbf{- 1}}\right)$ \\
\hline Na-hiSi-Seq & 33.51 & 3.9 & 0.78 \\
Na-hiSi & 27.29 & 3.6 & 0.72 \\
H-Y zeolite & 43.36 & 1.2 & 0.24 \\
H-ZSM-5 & 21.03 & 0.6 & 0.12 \\
\hline
\end{tabular}

The geopolymer catalysts developed in this work have the additional advantage of facile synthesis from readily available and relatively inexpensive naturally-occurring raw materials, by comparison with the synthesis of other advanced catalysts which involves multiple steps and expensive reagents. Since the geopolymer synthesis does not involve the use of toxic reagents such as OSDAs, the resulting catalysts are more ecologicallyfriendly materials. All these factors indicate that these catalysts represent a new class of materials with advantages in terms of performance, cost, ease of synthesis and ecological friendliness.

\subsection{Conclusion}

This work reports the first use of inorganic aluminosilicate geopolymers as heterogeneous solid acid catalysts for the liquid-phase Beckmann rearrangement of cyclohexanone oxime to $\varepsilon$-caprolactam. The geopolymer catalysts are readily prepared at room temperature from natural clay and alkali silicates under alkaline 
conditions. The catalytic sites are generated within the structure of geopolymer by ion-exchange with $\mathrm{NH}_{4}{ }^{+}$followed by thermal treatment. The surface areas and pore dimensions of the catalysts can be varied by adjusting the starting composition of the inorganic polymers, and it is found that excellent catalytic conversion of the oxime is obtained using geopolymers with small surface areas and large pore diameters; however, these catalysts showed low selectivity towards the caprolactam product. This was ascribed to the presence of Lewis acid sites generated by the thermal treatment which produced EFAl species. Nevertheless, the selectivity towards the $\varepsilon$-caprolactam can be significantly improved by exposing the geopolymer catalysts to a post-synthetic dealumination and desilication treatment which increases the surface area which we suggest enhances the distribution of the active sites. The catalytic activity of the geopolymers in the liquid-phase Beckmann rearrangement is superior to, or at least comparable with other solid acid catalysts under identical reaction conditions. These geopolymer-based catalysts were also recycled five times without deterioration in the yield of $\varepsilon$-caprolactam, emphasising their potential as efficient environmentally-friendly inexpensive heterogeneous catalysts as alternatives to the solid acids presently used in Beckmann rearrangement reactions.

The results reported in this chapter also show the feasibility of generating reactive Lewis acidic sites by applying a suitable thermal treatment to the geopolymer. This raises the possibility for the application of geopolymers as active catalysts in other organic reactions requiring Lewis acid sites. This aspect of these materials is investigated in the following chapter. 


\section{Chapter 4}

\section{Friedel-Crafts alkylation reactions catalysed over geopolymer catalysts}

\subsection{Introduction}

In the previous chapter it was shown that geopolymers could be synthesised with high surface areas and active sites, including weakly acidic silanol groups and minor amounts of Bronsted acid sites which could successfully be generated on the surface of the geopolymer, giving it high activity and selectivity for the Beckmann rearrangement of cyclohexanone oxime to $\varepsilon$-caprolactam.

In addition, it was also shown that Lewis acidic sites could be generated during thermal treatment, the concentration of which depended mainly on the temperature of the thermal treatment. These sites were active in facilitating side reactions resulting in the formation other by-products.

In this chapter, porous geopolymers were modified and their catalytic activities were evaluated for application to the industrially important Friedel-Crafts alkylation reactions where both Bronsted and Lewis acid sites are required.

Friedel-Crafts reactions are one of the oldest methods of introducing substituents into aromatics, demonstrated in 1887 by Charles Friedel and James Crafts for the synthesis of amyl benzene from benzene and amyl chloride using $\mathrm{AlCl}_{3}$ as a catalyst ${ }^{[143]}$. The Friedel-Crafts reaction, which can be used either for alkylation or acylation, is one of the most important industrial organic reactions; for instance, the global market revenue 
of the production of ethyl benzene (produced by alkylation of benzene with ethylene) accounted for 18.28 billion USD in 2015 with an expected rise to 23.31 billion USD by $2021^{[144]}$. Such reactions are catalysed mainly by conventional homogenous Lewis acids such as $\mathrm{AlCl}_{3}, \mathrm{H}_{2} \mathrm{SO}_{4}, \mathrm{HF}$, or $\mathrm{ZnCl}_{2}$, but these catalysts possess drastic drawbacks in terms of toxicity, corrosiveness, handling difficulty and reusability ${ }^{[11,39]}$.

Zeolites such as HZSM-5 and more recently MCM-22 have successfully replaced the homogenous catalysts, and are now commercially used in applications involving alkylation reactions including the production of ethylbenzene, cumene (by alkylation of benzene with propylene), and $p$-ethyltoluene. The latter is used as a precursor for production of poly- $p$-methylstyrene, a material that possesses superior properties to polystyrene ${ }^{[12,145]}$.

On the other hand, zeolites are not very efficient in the Friedel-Crafts alkylation of aromatics with benzyl substituents (benzylation reactions using benzylhalide or benzyl alcohol as the alkylating agent) (scheme 4.1). Although some microporous zeolites such as the beta-form have been used in this reaction and show high reactivity in the alkylation of benzene with benzyl alcohol, they show poor reactivity in the alkylation of relatively larger molecules such as substituted benzenes due to their restricting micropores ${ }^{[27 b]}$.

Scheme 4.1 Friedel-Crafts alkylation of benzene or substituted benzene with benzyl halide.

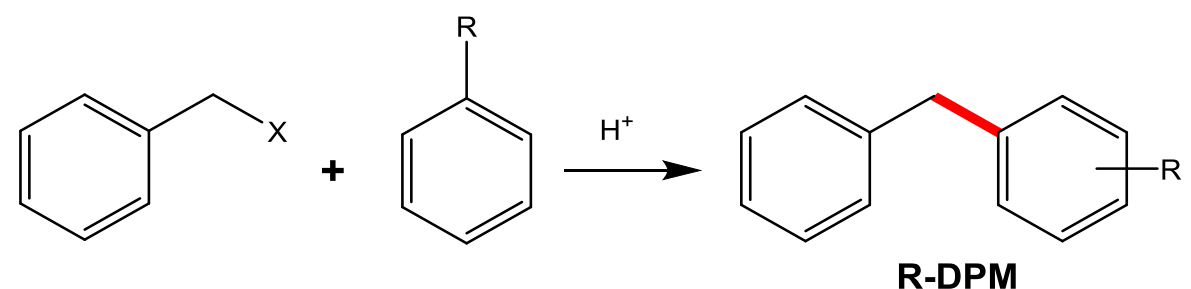

Aromatic benzylation is an important type of organic reaction, being used to synthesise a number of industrially valuable synthetic intermediates for the production of pharmaceuticals and fine chemicals ${ }^{[146]}$. For instance, diphenylmethane (DPM) or substituted DPM (such as benzyltoluene) are very important industrially, with wide applications as pharmaceutical intermediates 
(Figure 4.1), heat-transfer fluids, dyes and fragrances ${ }^{[147]}$. They have also been used to improve the thermal stability of polyester, in adhesion and insulating applications, and as curing agents for epoxy resins ${ }^{[146]}$.

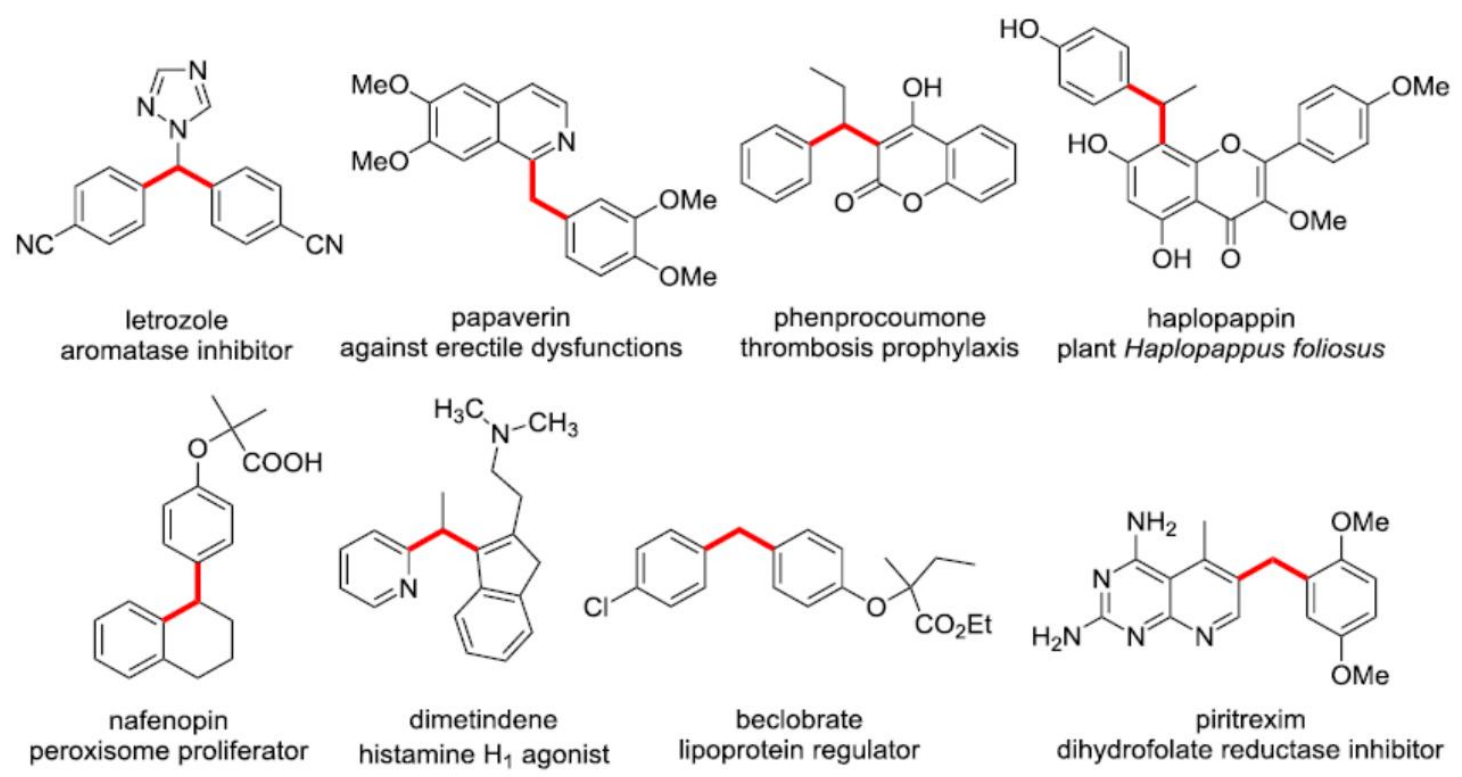

Figure 4.1 Several diaryalkanes and their biological activities ${ }^{[143]}$.

This class of reaction is still catalysed predominantly by the use of traditional homogenous Lewis acids. For this reason, several mesoporous solid catalysts have been developed to replace their homogenous analogues; these new catalysts include mesoporous zeolites ${ }^{[27 \mathrm{~b}, 148]}$, mesoporous silicates ${ }^{[149]}$, supported oxides ${ }^{[150]}$, and ionic liquids ${ }^{[151]}$.

Various active sites can catalyse the Friedel-Crafts alkylating reaction, depending on the alkylating agent used. For instance, Lewis acid sites are required with benzyl halides while Bronsted acid sites are needed with benzyl alcohol ${ }^{[10]}$.

The Friedel-Crafts alkylation reaction is an electrophilic substitution reaction, in which the initial step involves the formation of the benzyl carbocation $\left(\mathrm{C}_{6} \mathrm{H}_{5} \mathrm{CH}_{2}{ }^{+}\right)$ electrophile in the presence of a Bronsted and/or Lewis acid. The electrophile then attacks the activated benzene or substituted benzene to form the product (Figure 4.2) ${ }^{[152]}$. The reaction can also proceed via a free radical mechanism when an M-support catalyst is used. This mechanism will be discussed in the following chapter. 
In this chapter, geopolymers were prepared and modified so as to contain both Bronsted and Lewis acidic sites within their structure, as required to catalyse reactions such as Friedel-Crafts alkylations. Their catalytic activity was evaluated in liquid-phase Friedel-Crafts alkylation of a series of relatively large substituted benzenes (alkylation of toluene, anisole, p-xylene and mesitylene) using benzyl chloride $(\mathrm{BzCl})$ as the alkylating agent. The present work also investigated the effect of postsynthetic dealumination and desilication treatments on the structural and acidic properties and thus the improvement of the catalytic activities of these geopolymerbased catalysts.

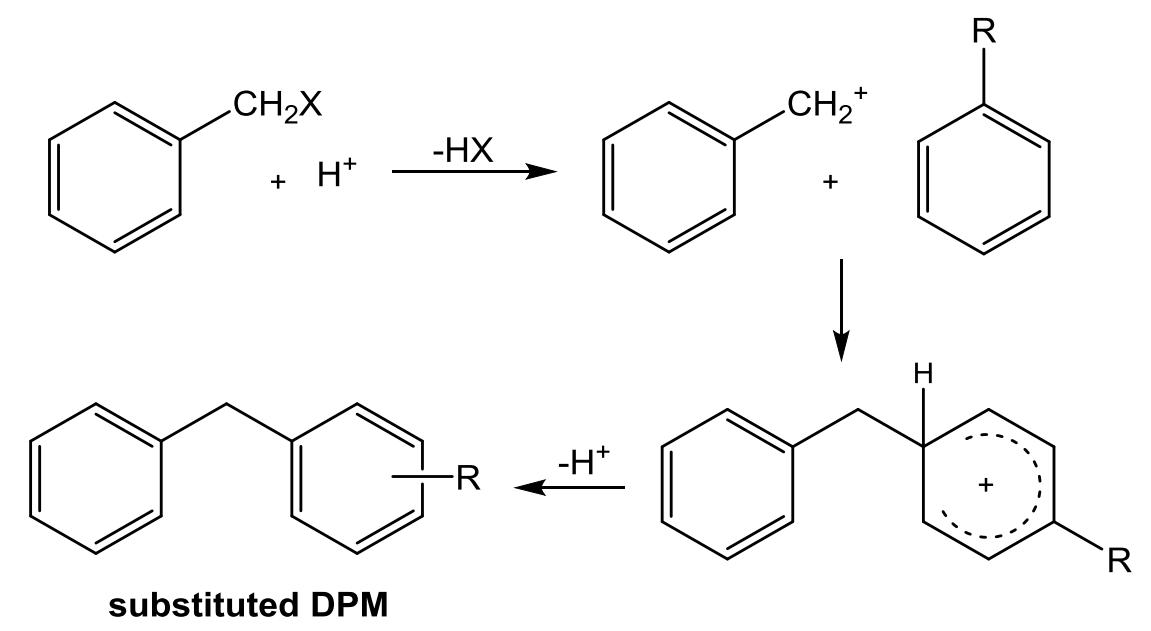

Figure 4.2 A mechanism of Friedel-Crafts alkylation of benzene or substituted benzene with benzylhalide as alkylating agent.

\subsection{Experimental}

\subsubsection{The catalyst preparation}

The catalysts used for the Friedel-Crafts alkylation reaction are similar to those reported in Chapter 3, but with different thermal pre-treatment. As in Chapter 3 the catalysts were the $\mathrm{NH}_{4}{ }^{+}$ion-exchanged form of the geopolymers, but as described in section 2.1.2 were heated to $550{ }^{\circ} \mathrm{C}$ to generate the required acid sites and held for 15 min prior to the reaction. The acidity measurements reported in this chapter were of these $\mathrm{NH}_{4}{ }^{+}-$ geopolymers heated at $550{ }^{\circ} \mathrm{C}$. 
In this work, the catalytic performance of Y zeolite was also determined for comparison purposes, by being treated in exactly the same manner as the geopolymer catalysts in terms of ion-exchange and thermal treatment.

\subsubsection{Catalytic reaction}

The reaction system is as described in chapter 2 (section 2.3.1). In a typical run, $1 \mathrm{ml}$ of $\mathrm{BzCl}$ was mixed with $13 \mathrm{ml}$ of the aromatic compound (Analytical grade toluene, anisole, p-xylene or mesitylene). $0.1 \mathrm{~g}$ of the catalyst was added to the reaction mixture at $85^{\circ} \mathrm{C}$ and the temperature was raised to $110^{\circ} \mathrm{C}$, this being taken as the starting time of the reaction. Calibration curves covering the range of $0.027-0.005 \mathrm{mmol} / \mathrm{ml}$ of each reactant and product were used for quantitative analysis. The reaction analyses and the catalytic calculations are described in Chapter 2 (section 2.33).

To test the reusability of the catalyst in Friedel-Crafts alkylation reaction, the catalyst was separated from the reaction mixture using a micro filter. The solid then was activated by heating to $550{ }^{\circ} \mathrm{C}$ for $1 \mathrm{hr}$ prior to each run. The structural stability of the spent catalyst was investigated by XRD.

\subsection{Results and discussion}

\subsubsection{Catalysts characteristics}

The structural characteristics of the synthesised geopolymers are described in detail in Chapter 2 (see section 3.3.1). To generate acid sites suitable for the Friedel-Crafts alkylation reactions, the geopolymer catalysts were heated to a higher temperature $\left(550^{\circ} \mathrm{C}\right)$ than was required for the Beckmann rearrangement. The structural stability and acidic properties of these catalysts are described below.

The structural stability of the heated geopolymer was evaluated by XRD as shown in Figure 4.3. The broadening of the original background hump (Figure 3.4) can be ascribed to the breakdown of the crystalline structure of some of the silicate or aluminate impurities in the geopolymer framework. Most importantly, no sign of recrystallization was seen, emphasising the thermal stability of the 
geopolymers at $550{ }^{\circ} \mathrm{C}$ even after heating for $5 \mathrm{hr}$. In addition, there was no noticeable effect of the thermal treatment on the sequentially treated catalysts compared with the untreated ones (see for example Figure $4.3 \mathrm{~d}$ and $4.3 \mathrm{e}$ which shows $\mathrm{Na}$-hiSi and $\mathrm{Na}$-hiSi-Seq respectively).

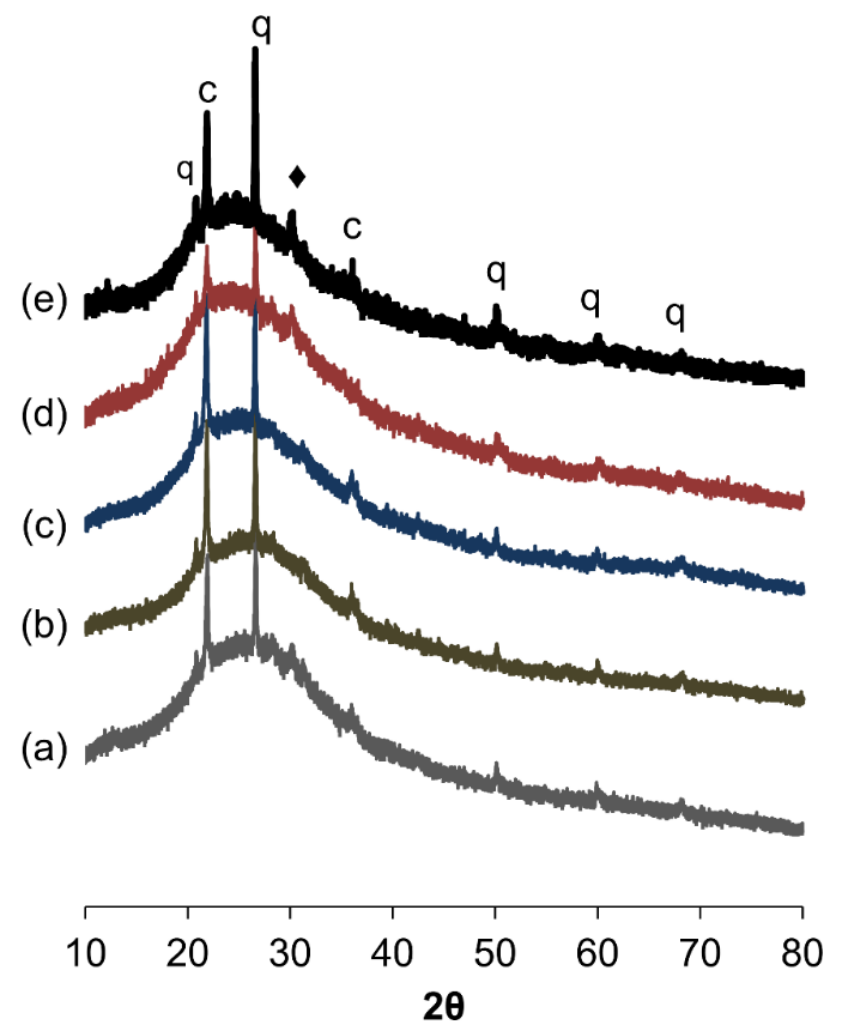

Figure 4.3 XRD traces of geopolymers thermally treated to $550{ }^{\circ} \mathrm{C}$ for $15 \mathrm{~min}$. (a) $\mathrm{K}$ hiSi, (b) K-N, (c) Na-N, (d) Na-hiSi, (e) Na-hiSi-Seq. (q: quartz; c: cristobalite; $\mathrm{Na}_{2} \mathrm{CO}_{3} \cdot \mathrm{H}_{2} \mathrm{O}$ )

The surface acidities of the geopolymer catalysts Na-hiSi and Na-hiSi-Seq are revealed in their FTIR spectra of adsorbed pyridine in Figure 4.4 which show the characteristic bands of the pyridine molecules coordinated with the Lewis acid sites (at 1448, 1600 and $1620 \mathrm{~cm}^{-1}$ ) and the Bronsted acid sites (at $1544 \mathrm{~cm}^{-1}$ ), and the combination of both sites (at $1490 \mathrm{~cm}^{-1}$ ), as discussed in section 2.2.6.2. These results show that heating at $550{ }^{\circ} \mathrm{C}$ generated higher surface acidity compared to heating at $450{ }^{\circ} \mathrm{C}$ (Fig 3.15). However, the total acidity is shown to be higher when the catalysts are only heated to $450{ }^{\circ} \mathrm{C}$, which might be ascribed to the severe destruction of structural hydroxyls that generate Bronsted acidity when heated at $550{ }^{\circ} \mathrm{C}$. 


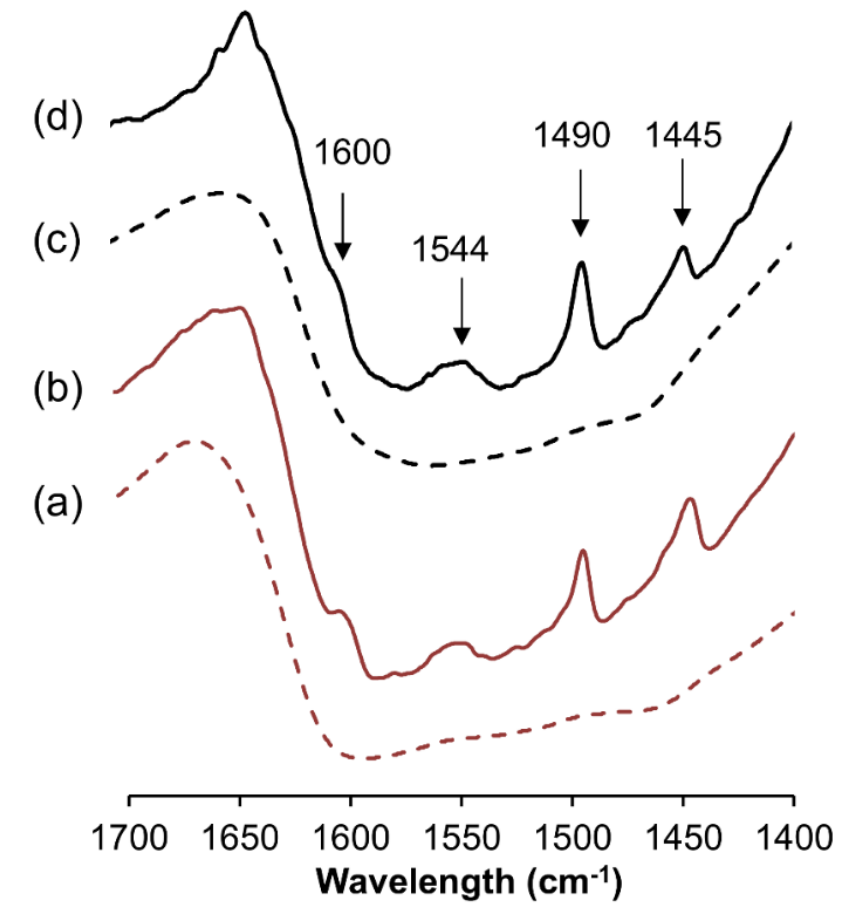

Figure 4.4 IR spectra of adsorbed pyridine over Na-hiSi-550 ${ }^{\circ} \mathrm{C}$ with and without sequential treatment; (a) Na-hiSi-550 ${ }^{\circ} \mathrm{C}$-background, (b) Na-hiSi-550 ${ }^{\circ} \mathrm{C}$-pyridine, (c) Na-hiSi-Seq-550 ${ }^{\circ} \mathrm{C}$-background, (d) Na-hiSi-Seq- $550{ }^{\circ} \mathrm{C}$-pyridine.

The TGA trace of the pyridine desorbed from these geopolymer catalysts is shown in Figure 4.5, with the corresponding values presented in Table 4.1.

Table 4.1 Total acid amount of the $\mathrm{NH}_{4}{ }^{+}$-ion exchanged geopolymers after heating to $550{ }^{\circ} \mathrm{C}$.

\begin{tabular}{lc}
\hline Catalyst & Acid content $(\mathrm{mmol} / \mathrm{g})$ \\
\hline K-N & 0.20 \\
K-N-Seq & 0.23 \\
K-hiSi & 0.18 \\
K-hiSi-Seq & 0.23 \\
Na-N & 0.14 \\
Na-N-Seq & 0.20 \\
Na-hiSi & 0.32 \\
Na-hiSi-Seq & 0.37 \\
\hline
\end{tabular}


Figure 4.5 shows that, as for the catalysts to $450{ }^{\circ} \mathrm{C}$ (Chapter 3, Figure 3.15), the sequentially treated catalyst shows a slightly higher amount of desorbed pyridine in the range $100-350{ }^{\circ} \mathrm{C}$, indicated by broken lines in Fig. 4.5.

(a)
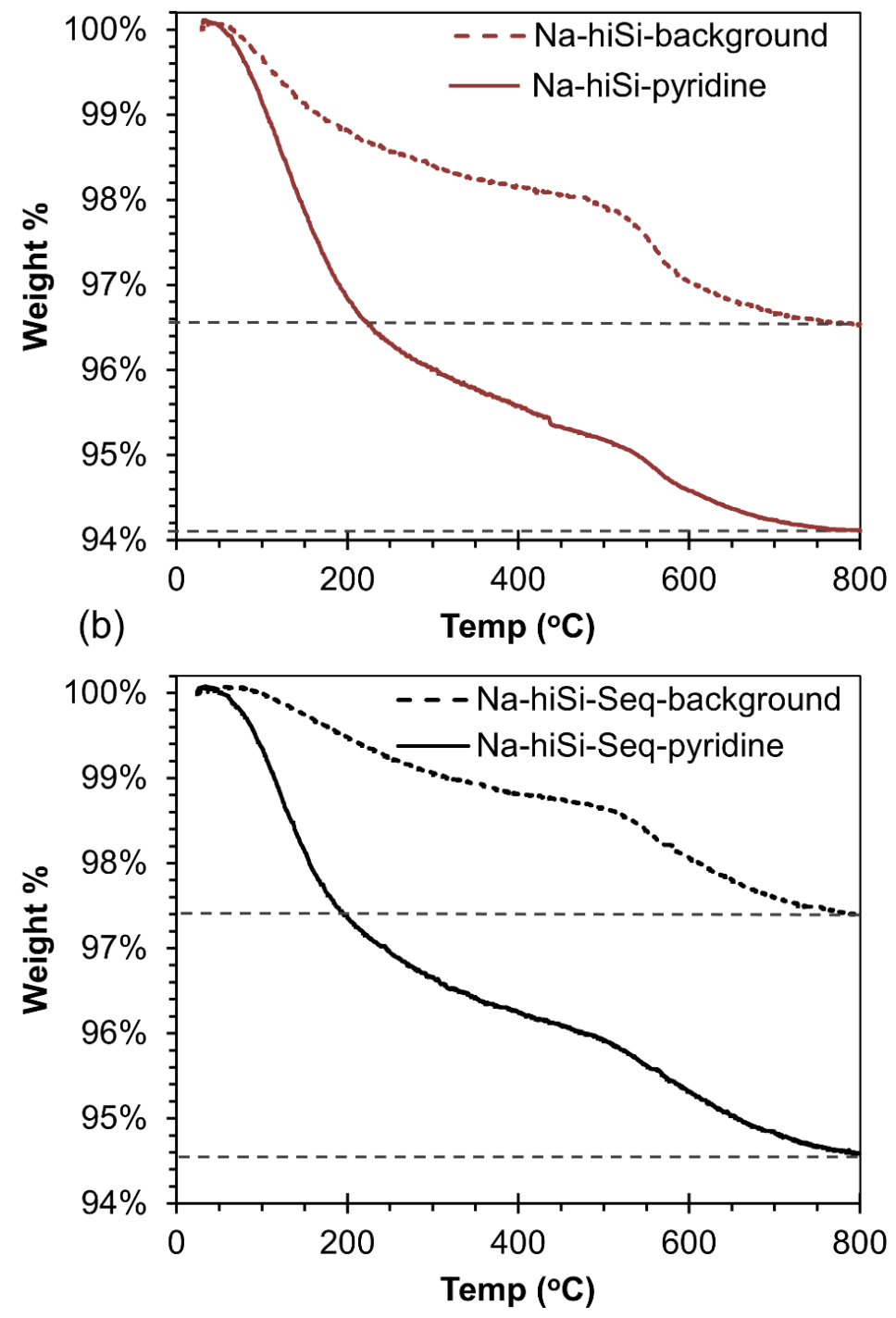

Figure 4.5 TGA profiles of pyridine degradation of representative catalysts that were heated to $550{ }^{\circ} \mathrm{C}$ for $15 \mathrm{~min}$. before and after sequential treatment. (a) Na-hiSi, (b) NahiSi-Seq.

The data in Table 4.1 and Figure 4.5 show that the total acidity of the catalysts heated at $550{ }^{\circ} \mathrm{C}$ is less than in the catalysts heated at $450{ }^{\circ} \mathrm{C}$ (see Table 3.1 and Figure 3.15). This can be ascribed to the severe dehydroxylation caused by heating the solids at 550 ${ }^{\circ} \mathrm{C}$, affecting the Bronsted acidic sites (the bridging $\mathrm{OH}$ groups) as shown in Figure 4.6. 


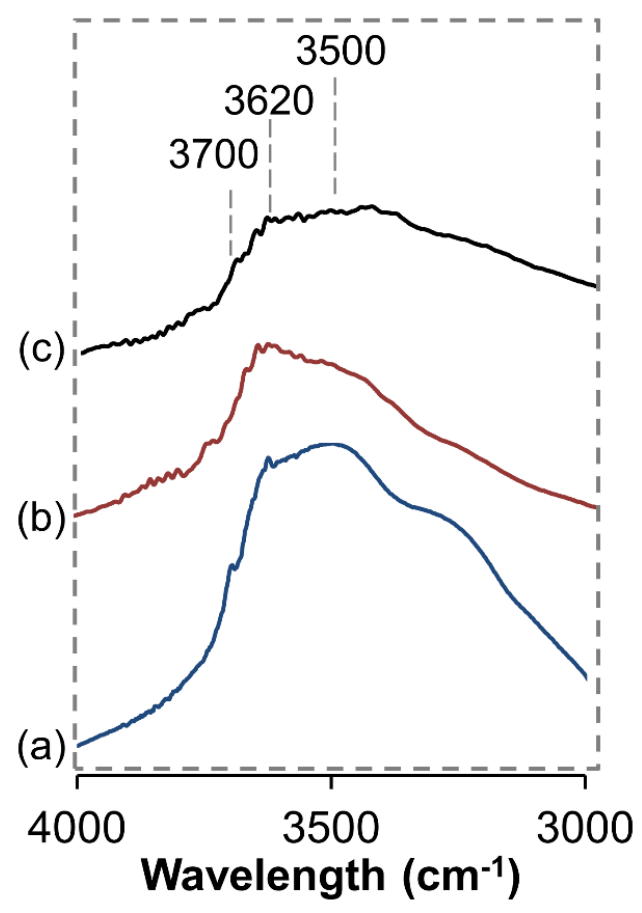

Figure 4.6 FTIR spectra in the $\mathrm{OH}$ region of $\mathrm{NH}_{4}{ }^{+}$-ion exchanged geopolymers with and without sequential treatment, after heating at different temperatures. (a) Na-hiSi$450{ }^{\circ} \mathrm{C}$, (b) Na-hiSi-550 ${ }^{\circ} \mathrm{C}$, (c) Na-hiSi-Seq $550^{\circ} \mathrm{C}$.

Figure 4.6 shows a higher concentration of hydroxyl groups (particularly bridging $\mathrm{OH}$ at $3620 \mathrm{~cm}^{-1}$ ) in sample Na-hiSi-450 ${ }^{\circ} \mathrm{C}$ (Figure 4.6a) compared with the same catalyst heated at $550{ }^{\circ} \mathrm{C}$ (Na-hiSi-550 ${ }^{\circ} \mathrm{C}$, Figure 4.6b). Greater destruction of the hydroxyl groups occurred in the catalyst heated at $550{ }^{\circ} \mathrm{C}$ after sequential treatment (Figure 4.6c), but the sequentially treated catalysts still show higher acidity than the untreated ones (Table 4.1).

The effect of the sequential treatment was investigated further by comparing the influence of different stages of the sequential treatment in geopolymers of two different $\mathrm{Si} / \mathrm{Al}$ molar ratios (Figure 4.7).

Geopolymers with the typical Si/Al molar ratio of $\sim 3.5$ (e.g. Na-N) show the generation of extra-framework aluminium (EFAl) during the dealumination step (Figure 4.4b), as evidenced by the two resonances at about $30.5 \mathrm{ppm}$ and $3.1 \mathrm{ppm}$ corresponding to penta-coordinated and octahedral Al respectively ${ }^{[153]}$. After completion of the sequential treatment, Na-N-Seq (Figure 4.4c) shows a similar 
spectrum to the starting material in which the EFAl species were removed, and the tetrahedral peak is shifted back to $\sim 60 \mathrm{ppm}$ due to desilication.

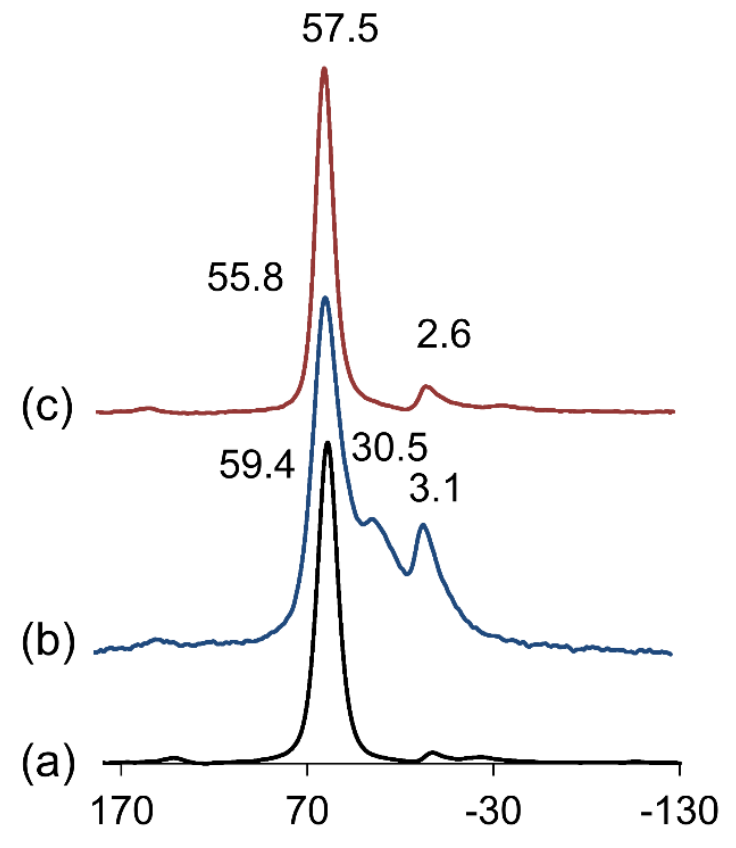

${ }^{27} \mathrm{Al} \mathrm{shift} \mathrm{(ppm)} \mathrm{w.r.t.} \mathrm{Al}\left(\mathrm{H}_{2} \mathrm{O}\right)_{6}{ }^{3+}$

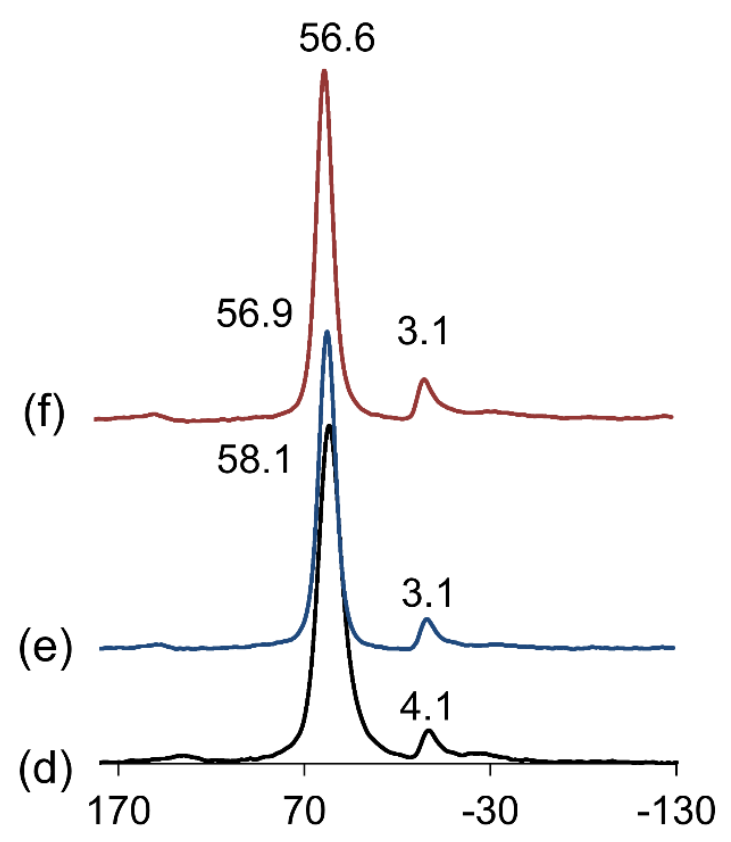

${ }^{27} \mathrm{Al}$ shift (ppm) w.r.t. $\mathrm{Al}\left(\mathrm{H}_{2} \mathrm{O}\right)_{6}{ }^{3+}$

Figure 4.7 Representative ${ }^{27} \mathrm{Al}$ MAS NMR spectra of geopolymer catalysts with different compositions at different stages of post-synthetic treatments. (a-c) Na-N; (a) Na-N parent, (b) Na-N-DA, (c) Na-N-Seq, (d-f) Na-hiSi; (d) Na-hiSi parent, (e) NahiSi-DA, (f) Na-hiSi-Seq.

By contrast, geopolymers with a higher $\mathrm{Si}$ content $(\mathrm{Si} / \mathrm{Al}$ molar ratio $\sim 6.5$, e.g. $\mathrm{Na}$-hiSi) appear to be more resistant to the acid and the sequential treatments (Figures $4.4 \mathrm{e}$ and $4.4 \mathrm{f}$ respectively).

However, by heating the catalysts at $550{ }^{\circ} \mathrm{C}$ prior use in the catalytic reactions, all the sequentially-treated catalysts show larger amounts of EFAl species (Figures $4.8 \mathrm{e}-\mathrm{h}$ ) compared with catalysts that had not been sequentially treated (Figures 4.8a-d). This explains the higher acidities of the sequentially-treated catalysts compared with their untreated analogues. 

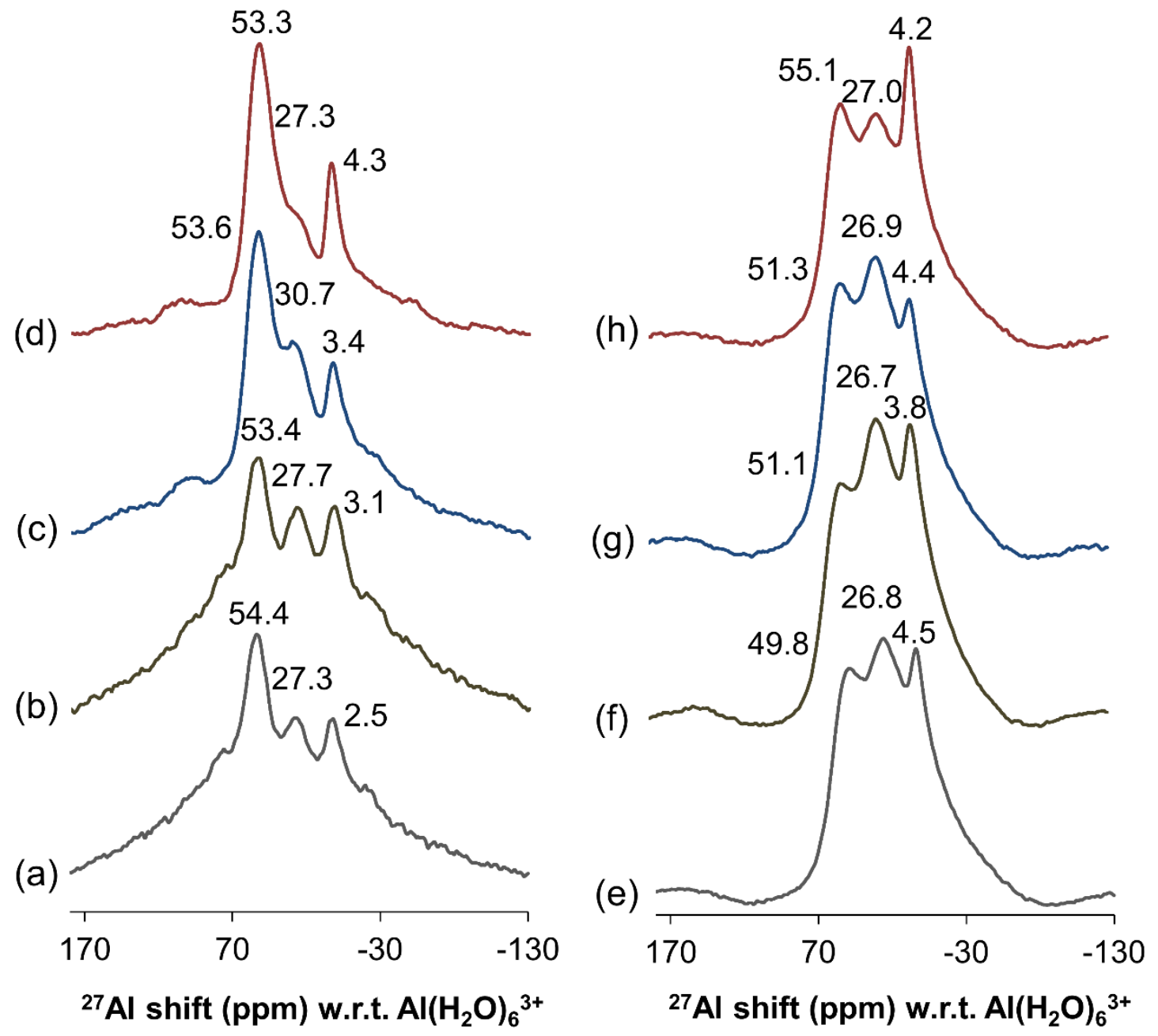

Figure 4.8 ${ }^{27} \mathrm{Al}$ MAS NMR spectra of heated geopolymer catalysts heated at $550{ }^{\circ} \mathrm{C}$ without sequential treatment (a-d); (a) K-hiSi, (b) K-N, (c) Na-N, (d) Na-hiSi, and with sequential treatment (e-h); (e) K-hiSi-Seq, (f) K-N-Seq, (g) Na-N-Seq, (h) Na-hiSi-Seq.

\subsubsection{Catalytic Reactivity}

In the first instance, the catalytic reactivity of the geopolymer catalysts was investigated using the benzylation of toluene with $\mathrm{BzCl}$ as the model Friedel-Crafts reaction. The results are shown in Figure 4.9. The as-synthesised catalysts show poor reactivity, with a conversion of $\mathrm{BzCl}$ of less than $30 \%$. The dealuminated geopolymer catalysts, on the other hand, show slightly higher reactivity, with conversions of up to $\sim 50 \%$ in some cases. 

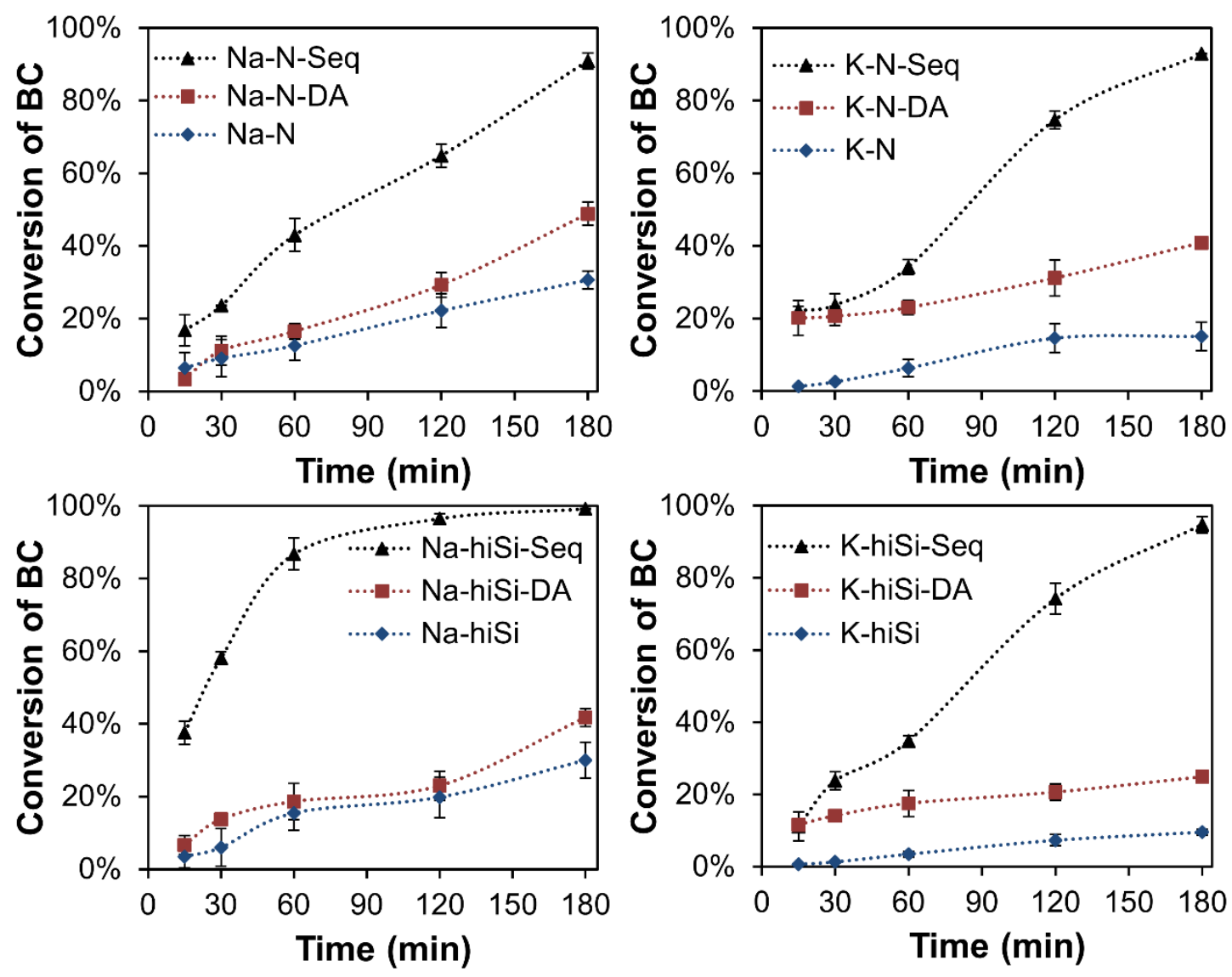

Figure 4.9 Catalytic reactivity of the various geopolymer catalysts in alkylation of toluene with $\mathrm{BzCl}$. Reaction conditions: $13.0 \mathrm{ml}$ toluene; $1.0 \mathrm{ml} \mathrm{BzCl} ; 0.1 \mathrm{~g}$ catalyst; $\mathrm{T}$ $110^{\circ} \mathrm{C}$.

However, after desilication followed by acid washing (the sequential treatment), a significant improvement of the catalytic reactivity was achieved, the reaction being almost complete within $3 \mathrm{hr}$. (Table 4.2), and with $100 \%$ selectivity towards monobenzylated toluene (BzTol). These results indicate that sequential treatment is essential to obtain optimal catalytic performance. This very significant improvement can be attributed to both the enhanced acidity, as discussed above, and the improved porosity (Table 3.1) which provides sufficient access of the reactant to the active sites in the pores of the solid and facilitates the removal of the products from the pores. This highlights the important role of desilication followed by washing with disodium EDTA salt in the improvement of the porosity and the acidity, thereby leading to higher catalytic reactivity. 
Of the present catalysts, Na-hiSi-Seq shows superior reactivity, achieving $\sim 90 \%$ conversion of $\mathrm{BzCl}$ within 1hr. (Figure 4.9). Compared with the other geopolymer catalysts, Na-hiSi-Seq has higher acidity as well as unique hierarchical porosity (Figure 3.11b). These superior results appear to be related to its composition (Table 2.2) which seems to be optimal for catalyst geopolymers.

Table 4.2 indicates that all the sequentially treated catalysts show comparable catalytic reactivity to $\mathrm{H}-\mathrm{Y}$ zeolite in terms of conversion and reaction rate; however, the TON and TOF values of all the geopolymers are several times higher than for $\mathrm{Y}$-zeolite, indicating a greater efficiency and superior catalyst lifetime of the geopolymer catalysts. In addition, the synthesis of the geopolymer catalysts is a much more energy-efficient and simpler procedure which does not involve lengthy thermal treatments or the use of costly and sometimes toxic, OSDAs that are needed for zeolite synthesis, see Section 1.3.1.

Table 4.2. Catalytic reactivity of several sequentially treated geopolymer-based catalysts in alkylation of toluene with benzylchloride ${ }^{\text {a }}$

\begin{tabular}{|c|c|c|c|c|c|c|}
\hline \multirow{2}{*}{ Catalyst } & \multirow{2}{*}{$\begin{array}{c}\text { Conversion } \\
\%\end{array}$} & \multicolumn{2}{|c|}{ Selectivity \% } & \multirow{2}{*}{$\begin{array}{c}\text { Specific } \\
\text { reaction } \\
\text { rate }^{b}\end{array}$} & \multirow{2}{*}{ TON } & \multirow{2}{*}{$\underset{\left(\mathbf{m i n}^{-1}\right)}{\text { TOF }}$} \\
\hline & & $p$-BzTol & $o$ - BzTol & & & \\
\hline K-N-Seq & 93 & 43 & 57 & 26.94 & 351 & 1.95 \\
\hline K-hiSi-Seq & 95 & 54 & 46 & 27.47 & 358 & 1.99 \\
\hline $\mathrm{Na}-\mathrm{N}-\mathrm{Seq}$ & 90 & 47 & 53 & 26.16 & 392 & 2.18 \\
\hline Na-hiSi-Seq & 99 & 52 & 48 & 29.53 & 231 & 1.29 \\
\hline$H-Y^{c}$ & 92 & 59 & 41 & 26.77 & 45 & 0.25 \\
\hline
\end{tabular}

${ }^{\text {a }}$ Reaction condition; $13.0 \mathrm{ml}$ toluene, $1.0 \mathrm{ml} \mathrm{BzCl}, 0.1 \mathrm{~g}$ catalyst; $\mathrm{T}=110{ }^{\circ} \mathrm{C} ; \mathrm{t}=3 \mathrm{hr}$. ${ }^{\mathrm{b}} \mathrm{mmol}_{\mathrm{BzCl}} \cdot \mathrm{g}_{\mathrm{cat}}{ }^{-1} \cdot \mathrm{h}^{-1}$

${ }^{\mathrm{c}}$ see section 3.2.1 for catalysts preparation.

The reaction kinetics were studied for the Na-hiSi-Seq catalyst in the alkylation of toluene (Figures 4.10 and 4.11). The reaction fits the Langmuir-Hinshelwood 
pseudo-first-order kinetic model with $\mathrm{R}^{2}>0.996$ (Figure 4.10). From the slope of the linear plot, the reaction constant $(\mathrm{k})$ was found to be $0.027 \mathrm{~min}^{-1}$.

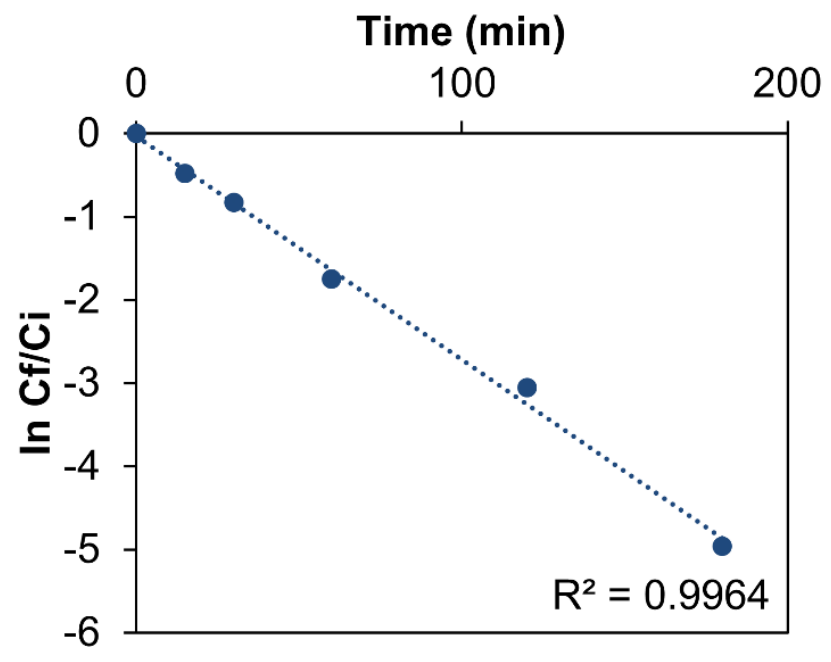

Figure 4.10. Langmuir-Hinshelwood pseudo-first-order kinetic model and for alkylation of toluene with $\mathrm{BzCl}$ over $\mathrm{Na}$-hiSi-Seq.

The influence of temperature on the reaction rate is described by the Arrhenius plot (Figure 4.11) from which the activation energy was determined to be 152 $\mathrm{kJ} / \mathrm{mol}$.

$1 / \mathrm{T}(\mathrm{K})$

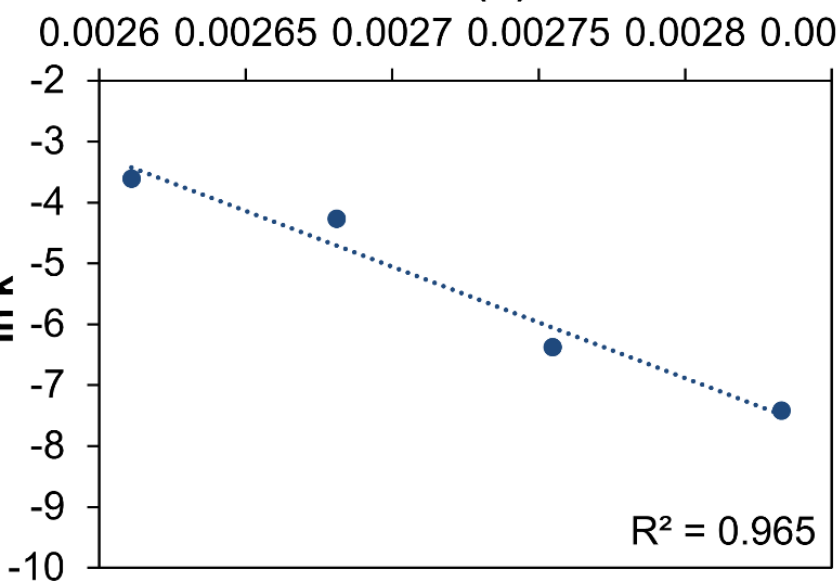

Figure 4.11 Arrhenius plot for alkylation of toluene with $\mathrm{BzCl}$ over Na-hiSi-Seq.

This plot shows that the reaction rate constant is proportional to the temperature, in agreement with the literature regarding alkylation of benzene or a substituted benzene over various other solid catalysts ${ }^{[27 a, 154]}$. 
The value of the Weisz-Prater criterion ( $\left.\phi_{\mathrm{W} . \mathrm{P}}\right)$ parameter in the present study was found to be $7.43 \times 10^{-6}(<<0.3)$, which, on the basis of these criteria, indicates the absence of pore diffusion limitations ${ }^{[114]}$. All the values used in these calculations are summarised in Table 4.3, and all the relevant equations are described in Chapter 2, Section 2.3.3.4.

The influence of the substrate: catalyst weight ratio (wt. \%) for the Na-hiSi-Seq catalyst is shown in Figure 4.12. A higher degree of conversion was achieved when a larger amount of the catalyst was used; $~ 97 \%$ conversion of $\mathrm{BzCl}$ was achieved with 5:1 BzCl: catalyst wt. $\%$ in $1 \mathrm{hr}$., while a conversion of $\sim 90 \%$ and $\sim 75 \%$ was obtained with 10:1 and 20:1 wt.\% respectively.

This can be understood in terms of the greater number of active sites available when a larger amount of catalyst is present in the reaction mixture. However, the reaction is almost complete at $\sim 3 \mathrm{hr}$. even when $\mathrm{BzCl}$ : catalyst wt. $\%$ is as high as 20:1.

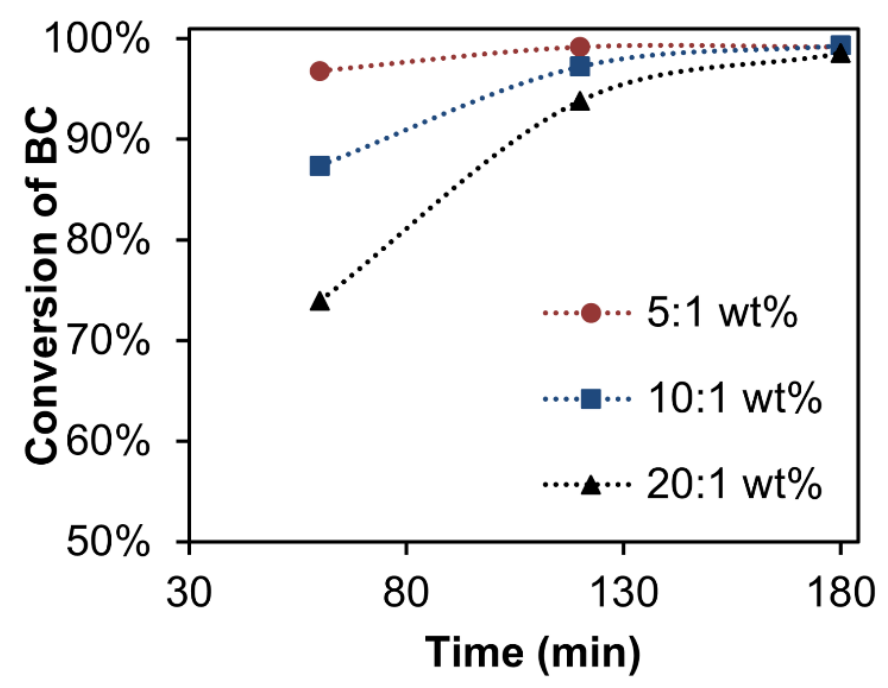

Figure 4.12 The influence of the substrate: catalyst wt. \% on the alkylation of toluene with $\mathrm{BzCl}$ over Na-hiSi-Seq catalyst. $\mathrm{T}=110{ }^{\circ} \mathrm{C}$. 
Table 4.3 Physical and chemical properties of the catalyst, solvent and reactant in alkylation of toluene with $\mathrm{BzCl}$ over $\mathrm{Na}-\mathrm{hiSi}_{-} \mathrm{Seq}^{\text {a }}$

\begin{tabular}{|c|c|c|}
\hline Variable & abbreviation & value \\
\hline Reaction rate $\left(\mathrm{mol} / \mathrm{cm}^{3} . \mathrm{s}\right)^{b}$ & $\mathrm{r}_{\mathrm{a}}$ & $1.3236 \times 10^{-5}$ \\
\hline Catalyst particle radius $(\mathrm{cm})$ & $\mathrm{R}_{\mathrm{p}}$ & $8.30 \times 10^{-4}$ \\
\hline Concentration of $\mathrm{BzCl}\left(\mathrm{mol} / \mathrm{cm}^{3}\right)$ & $\mathrm{C}_{\mathrm{a}}$ & $6.6847 \times 10^{-4}$ \\
\hline Effective diffusivity $\left(\mathrm{cm}^{2} / \mathrm{s}\right)$ & $D_{\text {eff }}$ & $2.9099 \times 10^{-5}$ \\
\hline Catalyst pore radius (nm) & $\mathrm{r}_{\text {pore }}$ & 8.19 \\
\hline Radius of $\mathrm{BzCl}(\mathrm{nm})^{[155]}$ & $\mathrm{r}_{\mathrm{BzCl}}$ & 0.4027 \\
\hline constant & $\mathrm{P}$ & 16.30 \\
\hline Bulk diffusivity $\left(\mathrm{cm}^{2} / \mathrm{s}\right)$ & $\mathrm{D}_{\mathrm{b}}$ & $5.7983 \times 10^{-5}$ \\
\hline Reaction temperature $(\mathrm{K})$ & $\mathrm{T}$ & 383.15 \\
\hline Association constant of toluene ${ }^{[117]}$ & $x$ & 1 \\
\hline Molar mass of toluene $(\mathrm{kg} / \mathrm{kmol})$ & $\mathrm{M}_{2}$ & 92.14 \\
\hline Viscosity of toluene at $110^{\circ} \mathrm{C}$ (Pa.s) ${ }^{[119]}$ & $\eta_{2}$ & $2.47 \times 10^{-4}$ \\
\hline Molar volume of $\mathrm{BzCl}\left(\mathrm{m}^{3} / \mathrm{kmol}\right)$ & $\mathrm{V}_{1}$ & $1.3532 \times 10^{-4}$ \\
\hline Molar mass of $\mathrm{BzCl}(\mathrm{kg} / \mathrm{kmol})$ & M & 126.58 \\
\hline Density of $\mathrm{BzCl}$ at room temperature $\left(\mathrm{kg} / \mathrm{m}^{3}\right)$ & $\mathrm{d}$ & $1.10 \times 10^{3}$ \\
\hline Room temperature (K) & $\mathrm{T}$ & 293.15 \\
\hline Boiling point of $\mathrm{BzCl}(\mathrm{K})$ & $\mathrm{T}_{\mathrm{b}}$ & 447.15 \\
\hline
\end{tabular}

${ }^{\mathrm{a}}$ Reaction condition; $13.0 \mathrm{ml}$ toluene, $1.0 \mathrm{ml} \mathrm{BzCl}, 0.1 \mathrm{~g}$ catalyst; $\mathrm{T}=110^{\circ} \mathrm{C} ; \mathrm{t}=3 \mathrm{hr}$. ${ }^{\mathrm{b}}$ Density of Na-hiSi-Seq $=0.80 \mathrm{~g} / \mathrm{cm}^{3}$.

The catalytic reactivity of $\mathrm{Na}-\mathrm{hiSi}$-Seq in the alkylation of other larger aromatics (anisole, p-xylene, and mesitylene) with $\mathrm{BzCl}$ is shown in Figure 4.13 and Table 3. Figure 4.13 shows that up to $30 \mathrm{~min}$, the reactivity increases in the following order: anisole $>$ p-xylene $>$ mesitylene $>$ toluene. These aromatics were tested to 
investigate the confinement effect of the geopolymer porosity when dealing with bulky molecules.

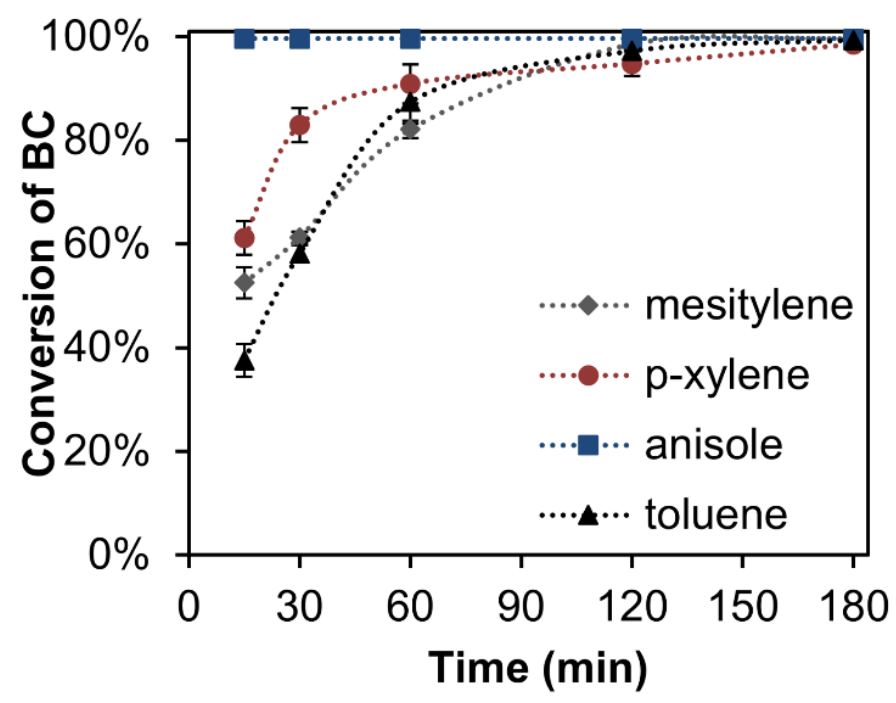

Figure 4.13 Catalytic reactivity of Na-hiSi-Seq in alkylation of mesitylene, p-xylene, anisole, and toluene with $\mathrm{BzCl}$. Reaction conditions: $13.0 \mathrm{ml}$ of each aromatic; $1.0 \mathrm{ml}$ $\mathrm{BzCl} ; 0.1 \mathrm{~g}$ catalyst; $\mathrm{T}=110{ }^{\circ} \mathrm{C}$.

The reaction with anisole was complete in less than $15 \mathrm{~min}$., and in the case of the other aromatics, the reaction was nearly complete after $\sim 3 \mathrm{hr}$, with $100 \%$ selectivity towards monobenzylated products (scheme 4.2).

Scheme 4.2 Monobenzylated products of Friedel-Crafts alkylation of several aromatics<smiles>COc1ccccc1Cc1ccccc1</smiles>

a1<smiles>COc1ccc(Cc2ccccc2)cc1</smiles>

a2<smiles>Cc1ccccc1Cc1ccccc1</smiles>

b1<smiles>Cc1ccc(Cc2ccccc2)cc1</smiles>

b2<smiles>Cc1cc(C)c(Cc2ccccc2)c(C)c1</smiles>

c<smiles>Cc1ccc(C)c(Cc2ccccc2)c1</smiles>

This is as expected for a typical acid-catalysed electrophilic substitution reaction in which increasing the electron density in the aromatic ring due to attachment of 
the electron-donating groups enhances the reaction rate. However, the lower reactivity of mesitylene after $30 \mathrm{~min}$. reaction time, compared with p-xylene and toluene (Table 4.4) may be explained in terms of a confinement effect in which the resulting monobenzylated mesitylene is larger than monobenzylated p-xylene and benzyltoluene, affecting the reaction rate. These aromatics were studied

Table 4.4 Catalytic activity of Na-hiSi-Seq in the alkylation of several aromatics at 1 hr. reaction time ${ }^{\text {a }}$

\begin{tabular}{ccccc}
\hline & Anisole & p-Xylene & Mesitylene & Toluene \\
\hline Conversion \% & 100 & 91 & 82 & 88 \\
$\begin{array}{c}\text { Specific reaction rate } \\
\left(\mathbf{m m o l}_{\mathbf{B z C l . g}} \mathbf{g}_{\mathbf{c a t}} \mathbf{- 1}^{\mathbf{- 1}}\right)\end{array}$ & 84.67 & 77.27 & 69.80 & 75.94 \\
\hline
\end{tabular}

${ }^{\mathrm{a}}$ Reaction condition; $13.0 \mathrm{ml}$ aromatic, $1.0 \mathrm{ml} \mathrm{BzCl}, 0.1 \mathrm{~g}$ catalyst; $\mathrm{T}=110{ }^{\circ} \mathrm{C} ; \mathrm{t}=60$ $\min$.

The results shown in Figure 4.13 suggest that the performance of the geopolymer catalysts is significantly better than that of regular microporous zeolites, which show poor reactivity for the liquid-phase alkylation of such large molecules ${ }^{[27]}$.

The reusability of the Na-hiSi-Seq catalyst (Figure 4.14) shows no sign of deactivation after five cycles of use of the same catalyst.

These results suggest that geopolymer-based catalysts can readily and simply be regenerated for reuse, and that furthermore, the geopolymer catalysts do not display the drawbacks of supported catalysts, which suffer from the weak interaction between the active species and the host, leading to loss of reactivity due to leaching of these active sites. 


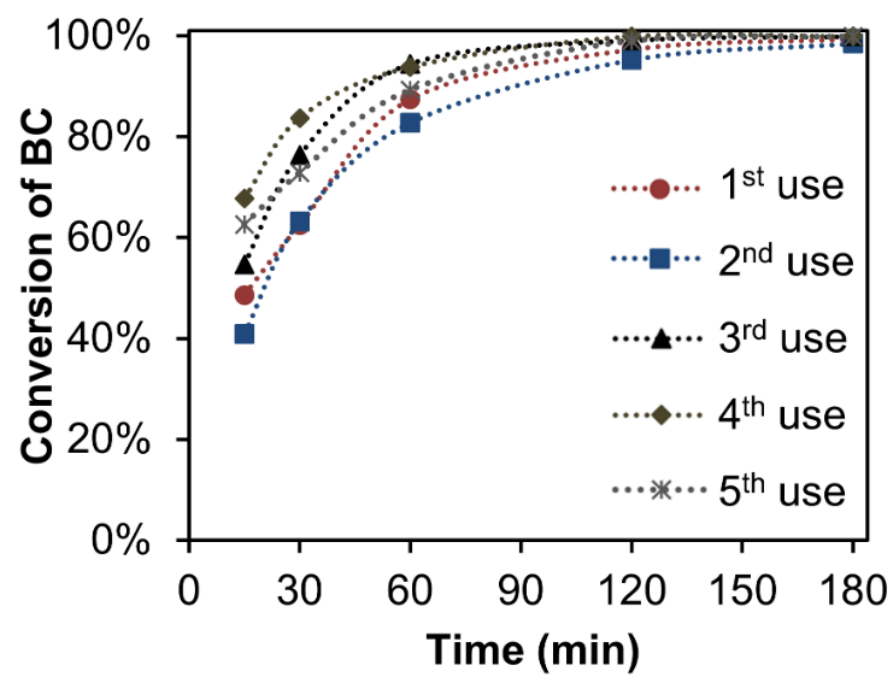

Figure 4.14 Reusability of Na-hiSi-Seq catalyst in alkylation of toluene with $\mathrm{BzCl}$. Reaction conditions; 130:10:1 Toluene: BzCl: catalyst wt. \%; T $110{ }^{\circ} \mathrm{C}$.

The stability of the reused geopolymer-based catalyst is illustrated in Figure 4.15 which shows little difference between the XRD pattern of the fresh and used catalyst after three reaction cycles, reflecting the high thermal and hydrothermal stability of the geopolymer-based catalysts.

(b)

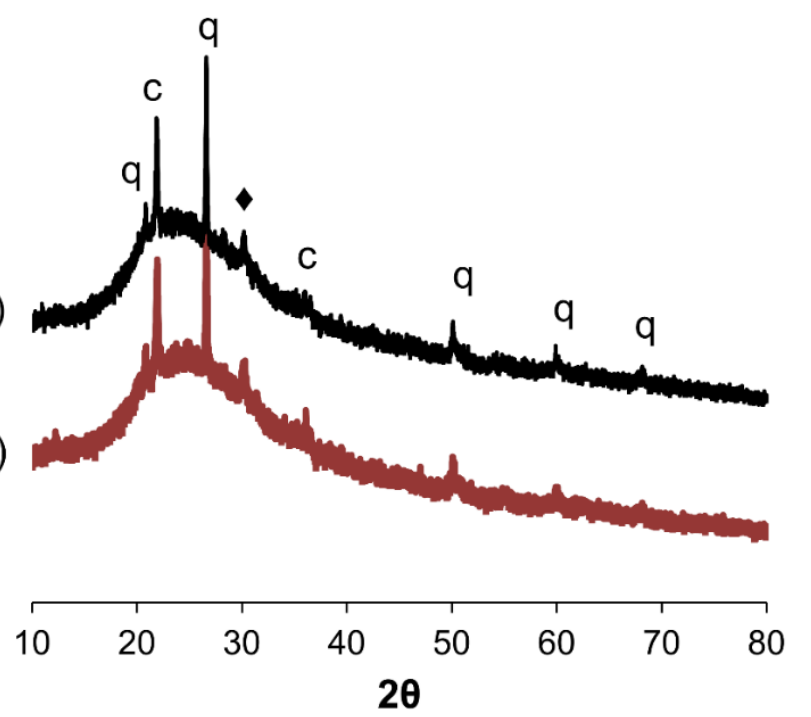

Figure 4.15 XRD traces of (a) fresh Na-hiSi-Seq catalyst and (b) the recovered catalyst after five reaction cycles. 


\subsection{Conclusion}

This chapter reports the heterogeneous catalytic applications of porous geopolymer-based catalysts in the Friedel-Crafts alkylation reactions of several substituted benzenes (toluene, anisole, p-xylene, and mesitylene) with benzyl chloride. The starting composition of the geopolymer was found to have a direct influence on the acidity and the porosity and thus the catalytic activity of the synthesised catalyst.

A very significant improvement in the reactivity of the as-synthesised catalysts was achieved by applying post-synthetic treatments (dealumination and desilication) which enhanced the acidity and porosity, resulting in a higher catalytic efficiency. The geopolymer catalyst could readily be regenerated and reused without significant loss of reactivity, since the acidic sites are generated within the geopolymer structure rather than supported on the geopolymer framework.

This Chapter further indicates the potential of geopolymers as new, cost efficient, readily synthesised and environmentally friendly heterogeneous solid acid catalysts for fine chemical applications, particularly for bulk compounds where regular microporous zeolites show poor reactivity. 


\section{Chapter 5}

\section{Activity of fly ash-based geopolymer catalysts in alkylation reactions}

\subsection{Introduction}

It was shown in the previous chapter that geopolymers based on a dehydroxylated clay (halloysite) could be prepared with meso, macro and hierarchical porosities and that Bronsted and Lewis acidic sites could be generated within their framework, making them capable of catalysing Friedel-Crafts alkylation of several aromatics. However, aluminosilicate clay is not the only starting material from which viable geopolymers can be synthesised; another well-known solid reactant for geopolymer synthesis is fly ash $^{[156]}$, the combustion product from coal-burning furnaces for example, in thermal power stations. Depending on the coal type from which it was derived, fly ash can contain a significant content of oxides such as $\mathrm{Fe}_{2} \mathrm{O}_{3}$, which are known to be very active catalysts for Friedel-Crafts alkylation reactions ${ }^{[157]}$ when used in conjunction with a solid supporting material. This suggests the possibility that a geopolymer synthesised from fly ash and containing $\mathrm{Fe}_{2} \mathrm{O}_{3}$ as one of the components, might demonstrate useful catalytic properties for Friedel-Crafts alkylation reactions. Such catalysts would have the additional economic and environmental advantages of being based on an industrial waste material rather than a clay mineral requiring additional energy for its dehydroxylation.

In this chapter, several geopolymer catalysts were synthesised from different fly ashes and their catalytic reactivities were tested in model Friedel-Crafts alkylation reactions. 


\subsubsection{Background to fly ash}

Fly ash is the solid waste of coal combustion, mainly in thermal power plants throughout the world. These coal combustion products (CCPs) represent the incombustible mineral matter (ash) present in the coal. The coal combustion products (CCPs), account for $\sim 20 \mathrm{wt} . \%$ of the feed coal ${ }^{[158]}$ and consist mainly of coarse bottom ash (the ash remaining in the bottom of the boiler during the combustion process), which represents $<15 \%$ of the total ash. The remaining ash, which accounts for $>85 \%$ of the total ash is called fly ash, and is the finer ash that is carried by the combustion gases through the boiler and collected by electrostatic precipitators at the boiler outlet ${ }^{[159]}$. Since the generation of electricity from coal combustion was introduced in 1920s, the global production of fly ash has grown continuously. The worldwide production of fly ash in 2000 was estimated to be around 349 million tonnes (Mt) ${ }^{[160]}$, increasing to about $430 \mathrm{Mt}$ in $2003^{[161]}$ and $780 \mathrm{Mt}$ in $201{ }^{[159]}$. Some of this fly ash is used in applications such as an extender for concrete; globally, $\sim 53.5 \%$ of the fly ash produced in 2010 was utilised (Table 5.1) and the remainder was disposed of by dumping into the sea or into landfills, causing serious environmental problems.

Table 5.1 Global production and utilisation of CCPs by country or region in $2010^{[159]}$.

\begin{tabular}{cccc}
\hline Country/Region & $\begin{array}{c}\text { CCPs Production } \\
(\mathbf{M t})\end{array}$ & $\begin{array}{c}\text { CCPs Utilisation } \\
(\mathbf{M t})\end{array}$ & $\begin{array}{c}\text { Utilisation Rate } \\
(\boldsymbol{\%})\end{array}$ \\
\hline Australia & 13.1 & 6.0 & 45.8 \\
Canada & 6.8 & 2.3 & 33.8 \\
China & 395.0 & 265.0 & 67.1 \\
Europe & 52.6 & 47.8 & 90.9 \\
India & 105.0 & 14.5 & 13.8 \\
Japan & 11.1 & 10.7 & 96.4 \\
Middle East \& & 32.2 & 3.4 & 10.6 \\
Africa & 118.0 & 49.7 & 42.1 \\
USA & 16.7 & 11.1 & 66.5 \\
Other Asia & 26.6 & 5.0 & 18.8 \\
\hline Russian Federation & $\mathbf{7 7 7 . 1}$ & $\mathbf{4 1 5 . 5}$ & $\mathbf{5 3 . 5}$ \\
\hline Total & & &
\end{tabular}


Fly ash contains a heterogeneous mixture of metal oxides, the majority being $\mathrm{SiO}_{2}$ and $\mathrm{Al}_{2} \mathrm{O}_{3}$. Other oxides present in minor amounts include $\mathrm{Fe}_{2} \mathrm{O}_{3}, \mathrm{TiO}_{2}, \mathrm{CaO}, \mathrm{MgO}, \mathrm{Na}_{2} \mathrm{O}$, and $\mathrm{K}_{2} \mathrm{O}$. The composition of fly ash is variable, depending on the source of the coal and the combustion technique. Table 5.2 shows the elemental composition range of fly ashes obtained from the combustion of three main coal types. Generally fly ash is classified according to the ASTM C618 standard into two classes based on the $\mathrm{CaO}$ content; F-class fly ash contains a low $\mathrm{Ca}$ content $(\mathrm{CaO}<8$ wt.\%) whereas $\mathrm{C}$-class fly ash contains $>8 \mathrm{wt} . \% \mathrm{CaO}$.

Table 5.2 Elemental composition (wt. \%) of fly ashes produced from the combustion of different coals ${ }^{[162]}$.

\begin{tabular}{cccc}
\hline Oxide & Bituminous Coal & Sub-bituminous Coal & Lignite \\
\hline $\mathrm{SiO}_{2}$ & $20-60$ & $40-60$ & $15-45$ \\
$\mathrm{Al}_{2} \mathrm{O}_{3}$ & $5-35$ & $20-30$ & $10-25$ \\
$\mathrm{Fe}_{2} \mathrm{O}_{3}$ & $10-40$ & $4-10$ & $4-15$ \\
$\mathrm{CaO}$ & $1-12$ & $5-30$ & $15-40$ \\
$\mathrm{MgO}$ & $0-5$ & $1-6$ & $3-10$ \\
$\mathrm{SO}$ & $0-4$ & $0-2$ & $0-10$ \\
$\mathrm{Na}_{2} \mathrm{O}$ & $0-4$ & $0-2$ & $0-6$ \\
$\mathrm{~K}_{2} \mathrm{O}$ & $0-4$ & $0-4$ & $0-4$ \\
$\mathrm{LOI}$ & $0-15$ & $0-3$ & $0-5$ \\
\hline
\end{tabular}

Nowadays, the development of appropriate disposal methods and bulk utilisation of fly ash is a worldwide concern, not only because of the immense amount that is produced but also because of its environmental hazards. The environmental burden of fly ash lies on the presence of other metals present in some fly ashes, including $\mathrm{Cr}, \mathrm{Pb}, \mathrm{Ni}, \mathrm{Ba}, \mathrm{Hg}$, $\mathrm{Zn}, \mathrm{Sr}$, As and V. In addition, traces of radioactive elements such as ${ }^{238} \mathrm{U},{ }^{40} \mathrm{~K},{ }^{226} \mathrm{Ra}$, and ${ }^{232} \mathrm{Th}$ may also be present in fly ash ${ }^{[158,163]}$. Although these metals are present in only trace amounts, the large quantities of fly ash continuing to be produced make them a serious risk to the environment and to human health. If the fly ash is dumped in landfills, these trace metals could leach out, contaminating the soil and the ground and 
surface water. Numerous studies have reported the impact of fly ash on soil and its negative effect on its fertility, microbial and enzymatic activity (see for example ref. $\left.{ }^{[163]}\right)$. The fine particles of fly ash may also have a direct impact on human health by inhalation, causing irritation to the throat and respiratory tract or even arsenic poisoning ${ }^{[158]}$. For these reasons, considerable efforts have been made to recycle fly ash into new products or utilise it in applications which would have the additional economic benefit of reducing disposal costs.

\subsubsection{Applications}

One of the major fields for utilising fly ash is in the construction industry, where $20 \%$ of fly ash production is incorporated in concrete manufacture due to its pozzolanic and cementitious properties ${ }^{[158]}$. Reported benefits of incorporating fly ash in cement include enhanced durability and elasticity of the concrete ${ }^{[164]}$. Other applications of fly ash in the construction sector include its use as an asphalt filler, pavement base course, structural and engineering fill, and soil stabiliser ${ }^{[164]}$. Recently, fly-ash-based geopolymer concrete has emerged as a highly promising environmentally-friendly replacement for cement-based concrete, exhibiting enhanced mechanical and chemical properties compared with regular cement-based concrete ${ }^{[165]}$.

Other applications to utilise fly ash have also been suggested, since increasing production is outstripping the demands of the civil engineering sector. Fly ash has been investigated as an adsorbent for flue gases and heavy metals in wastewater; fly ash treated with $\mathrm{CaO}$ has been found to be very reactive in capturing $\mathrm{SO}_{2}$, with full conversion of $\mathrm{CaO}$ to $\mathrm{CaSO}_{4}$ being achieved within one hour ${ }^{[160]}$. The removal of metals such as $\mathrm{Cu}, \mathrm{Pb}, \mathrm{Mn}, \mathrm{Cd}, \mathrm{Cr}$ and $\mathrm{Ni}$ from industrial waste water by fly ash has also recently been reported ${ }^{[166]}$. However, care should be taken when fly ash is used in water treatment due to the potential for metal leaching from the fly ash, causing secondary pollution ${ }^{[160]}$.

Fly ash has also been suggested as a source of valuable metals such as Ge, for use in a range of high-technology applications such as semiconductor manufacture ${ }^{[167]}$. The use of fly ash as a low cost precursor for other materials such as ceramics has also been suggested. Ceramic materials are usually produced from high grade raw materials, 
making them very expensive, but less-expensive ceramics can be synthesised from industrial by-products such as fly ash similar properties to regular ceramics ${ }^{[168]}$.

\subsubsection{Applications of fly ash in catalysis}

The presence of metals oxides such as $\mathrm{Fe}_{2} \mathrm{O}_{3}, \mathrm{TiO}_{2}, \mathrm{CaO}$ and $\mathrm{Na}_{2} \mathrm{O}$ makes fly ash a good potential candidate for catalysis applications. For this reason, several studies have reported the utilisation of fly ash in heterogeneous catalysis ${ }^{[161]}$. Fly ash has been used as a support for transition metals such as $\mathrm{Ni}, \mathrm{Fe}, \mathrm{Cu}$ in $\mathrm{CO}_{2}$ in syngas production ${ }^{[169]}$ and the reduction of $\mathrm{NO}_{\mathrm{x}}{ }^{[170]}$. Fly ash loaded with $\mathrm{KNO}_{3}$ was used for transesterification of vegetable oil for biodiesel production ${ }^{[171]}$ and sulfated zirconia supported on fly ash has recently been reported for catalysing the alkylation of benzene and toluene with benzylchloride ${ }^{[157 \mathrm{a}]}$. Fly ash as a support for $\mathrm{TiO}_{2}{ }^{[172]}$ and $\mathrm{Co}_{3} \mathrm{O}_{4}{ }^{[173]}$ has been tested in the photocatalytic degradation of phenol and shown to give superior results to the component oxides alone.

Fly ash has also been utilised in the synthesis of zeolites for waste water treatment and catalysis applications. Conventional hydrothermal syntheses fail to produce zeolite with the desired structural crystallinity due to the presence of impurities that are insoluble under alkaline conditions. Therefore, zeolite from fly ash precursors is usually synthesised by fusion of solid alkali with the fly ash at high temperatures $\left(>500{ }^{\circ} \mathrm{C}\right)$ followed by hydrothermal crystallisation ${ }^{[168 a]}$. Several zeolites have been synthesised from fly ashes and tested in a range of catalytic reactions, for example, zeolite $X^{[174]}$ and sodalite ${ }^{[175]}$ for the production of biodiesel, and zeolite $X$ in the alkylation of phenol, with results comparable with or superior to commercially available $\mathrm{X}$ zeolite ${ }^{[176]}$. Mesoporous silica (MCM-41) has also been synthesised from a fly ash precursor and tested as a catalyst for the Mannich reaction, giving results that were better than those obtained using acidic ionic liquids ${ }^{[177]}$. These mesoporous silicates were prepared in the same manner as zeolites, but a structural directing agent was added prior to or during the hydrothermal crystallisation step.

Very recently, geopolymers synthesised from fly ash have been tested as catalysts for the photo degradation of organic compounds in waste water ${ }^{[178]}$.

This chapter reports a facile synthesis of acidic fly ash-based geopolymer catalysts for liquid phase organic synthesis applications and investigates their catalytic properties 
for model Friedel-Crafts alkylation reactions of benzene and other substituted aromatics with benzylchloride as the alkylating agent. The catalysts were synthesised at room temperature in an environmentally friendly process without the need for alkali fusion or lengthy hydrothermal treatments. Fly ashes collected from different sources were used as received with no further pre-treatment, and the impact of the variation of the chemical and physical properties of the fly ash on the synthesised catalysts was studied.

\subsection{Experimental}

\subsubsection{Catalysts preparation}

The procedures for geopolymer synthesis from fly ash and the subsequent preparation steps of the catalysts are described in detail in Chapter 2 (sections 2.1.1.2 and 2.1.2). The compositions of the fly ashes are shown in Table 2.1.

The $\mathrm{NH}_{4}{ }^{+}$-forms of the fly ash-based geopolymers were heated to $550{ }^{\circ} \mathrm{C}$ and held for 15 min prior adding to the catalytic reaction. The zeolite $\mathrm{Y}$ used for comparison purposes was prepared exactly as described in Chapter 4 (section 4.2.1).

\subsubsection{Catalytic reaction}

The reaction system is as described in Chapter 2 (section 2.3.1). In a typical run, $1 \mathrm{ml}$ of $\mathrm{BzCl}$ was mixed with $13 \mathrm{ml}$ of the aromatic compound (benzene, toluene, anisole, p-xylene or mesitylene). $0.1 \mathrm{~g}$ of the catalyst was added to the reaction mixture at 80 ${ }^{\circ} \mathrm{C}$ which was taken as the starting time of the reaction. The reaction analysis and the catalytic calculations are described in Chapter 2 (section 2.33).

The reusability of the catalysts was investigated as described in Chapter 4 (section 4.2.2). The recycled catalyst was studied by TGA and FTIR.

\subsection{Results and discussion}

\subsubsection{Catalysts characteristics}

The geopolymers and the corresponding catalysts were analysed by XRD (Figure 5.1). Since fly ash is a product of a combustion process, all the as-received fly ashes display 
an amorphous feature (Figure 5.1a) and several crystalline phases; one of these was identified as a crystalline aluminosilicate such as mulite which probably formed due to heating at a very high temperature (up to $1700{ }^{\circ} \mathrm{C}$ ) allowing the silica and alumina to react. Other compounds present include $\mathrm{Fe}_{2} \mathrm{O}_{3}$ and $\mathrm{Ca}_{2} \mathrm{SiO}_{4}$.

$\triangle$ Mullite $\square$ Quartz $\bullet \mathrm{Fe}_{2} \mathrm{O}_{3} \times$ Sodalite $\left(\mathrm{Na}, \mathrm{NO}_{3}\right) \circ \mathrm{Ca}_{2} \mathrm{SiO}_{4}$
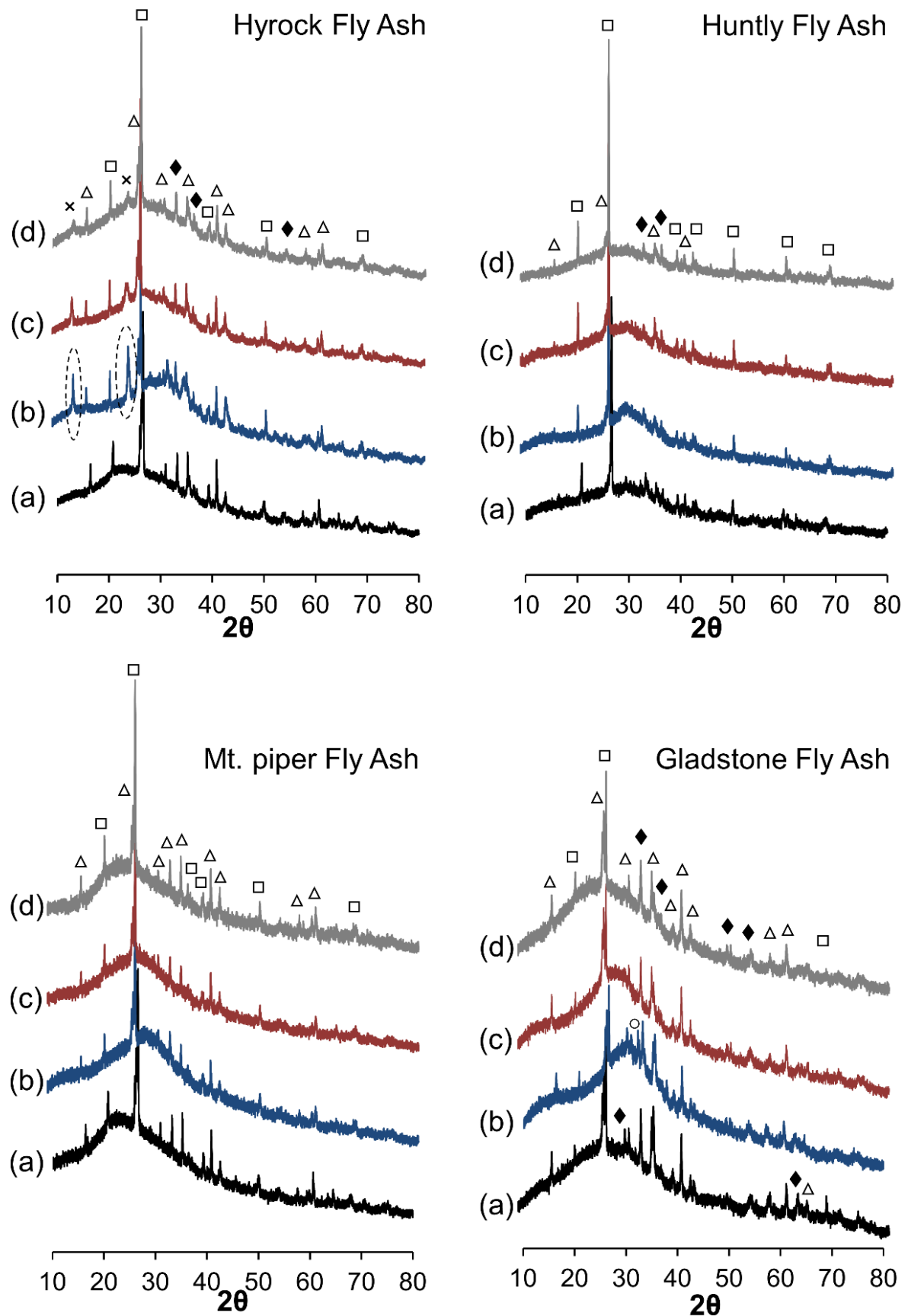

Figure 5.1 XRD of geopolymers prepared from different fly ashes. (a) raw fly ash, (b) fly ash-based geopolymer "as-synthesised", (c) $\mathrm{NH}_{4}{ }^{+}$-form of the geopolymer, (d) $\mathrm{NH}_{4}{ }^{+}$-geopolymer after heating to $550{ }^{\circ} \mathrm{C}$. 
The formation of the geopolymer is evidenced by the shift of the amorphous background of the fly ash to $>30^{\circ} 2 \boldsymbol{\theta}$. In some cases, a crystalline aluminosilicate (sodalite) was formed after geopolymerisation (circled peaks, Figure 5.1b-Hyrock fly ash). These peaks started to disappear after the ion-exchange step and had almost completely gone after heating to $550{ }^{\circ} \mathrm{C}$ (Figure $5.1 \mathrm{c}$ and $5.1 \mathrm{~d}$-Hyrock fly ash). Generally, no significant impact on the crystalline phases was observed in any of the fly ash-based catalysts after ion exchange with $\mathrm{NH}_{4}{ }^{+}$(Figure 5.1c). The amorphous background broadened after heating to $550{ }^{\circ} \mathrm{C}$ in the XRD traces of all the fly ash-based catalysts, which is ascribed to the removal of some of the crystalline impurities.

The ${ }^{27} \mathrm{Al}$ and ${ }^{29} \mathrm{Si}$ MAS NMR spectra of the fly ash based geopolymers did not provide useful information about their structure due to the presence of paramagnetic species such as $\mathrm{Fe}^{+2}$ and $\mathrm{Fe}^{+3}$, which result in line broadening and a poor signal-to-noise ratio. Although these spectra were acquired, they are not shown here.

Geopolymer formation was studied by FTIR. Figure 5.2 shows the spectra of all the fly ashes and the corresponding fly ash-based geopolymers. All the raw fly ashes show four main peaks (Figure 5.2a); that at about $795 \mathrm{~cm}^{-1}$ is ascribed to the octahedral AlO stretching vibration ${ }^{[179]}$ while the small peak at $\sim 561 \mathrm{~cm}^{-1}$ is ascribed to the symmetric stretching of $\mathrm{Si}-\mathrm{O}-\mathrm{Al}^{[137]}$ or to the $\mathrm{Fe}-\mathrm{O}$ stretching vibration which is usually in the range of $540-570 \mathrm{~cm}^{-1}{ }^{[180]}$. The main peak at $\sim 1100 \mathrm{~cm}^{-1}$ and the shoulder at $\sim 1160 \mathrm{~cm}^{-1}$ are typical of solid aluminosilicates and arise from the asymmetric Si-O-Al and Si-O-Si stretching modes respectively.

In all the as-synthesised geopolymers (Figure 5.2b), geopolymerisation is evidenced by the disappearance of the peak associated with the octahedral Al-O stretching mode at $795 \mathrm{~cm}^{-1}$. The other peaks are similar to those obtained from clay-based geopolymers (Figure 3.8). The asymmetric stretching modes of Si-O-Al and Si-O-Si remain in all the geopolymers, but the additional bands at $\sim 1480$ and $2339 \mathrm{~cm}^{-1}$ are associated with carbonation of the geopolymer surface by atmospheric $\mathrm{CO}_{2}$. The peaks at $\sim 3500$ and $3620 \mathrm{~cm}^{-1}$ are associated with silanol nests and bridging hydroxyls respectively. After $\mathrm{NH}_{4}{ }^{+}$-ion exchange (Figure 5.2c), all the geopolymers show the typical peaks of the $\mathrm{N}$ $\mathrm{H}$ stretching mode at $\sim 3200 \mathrm{~cm}^{-1}$ and the corresponding bending mode (the double peak at $\sim 1400$ and $1450 \mathrm{~cm}^{-1}$ ). 

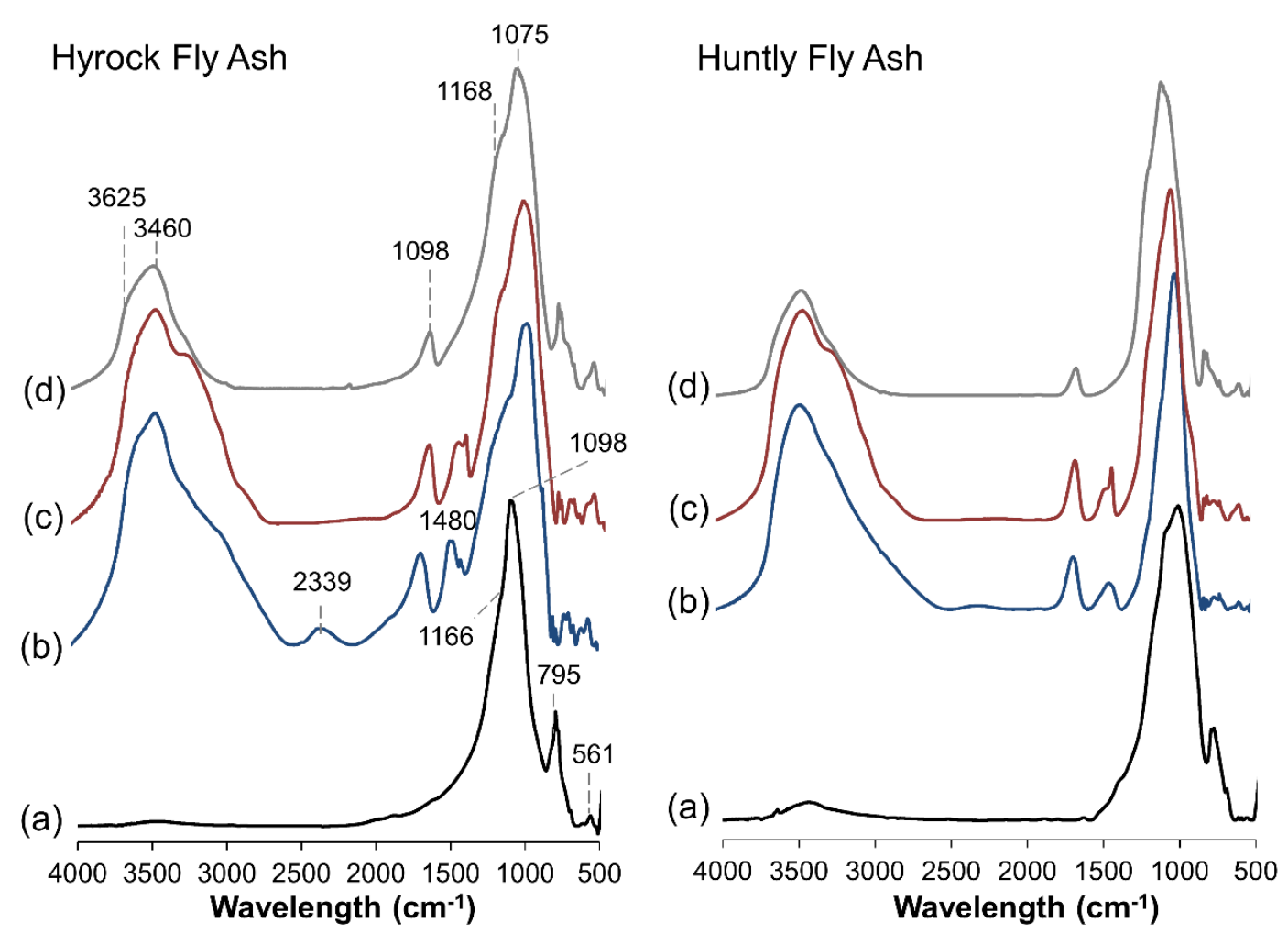

Mt. piper Fly Ash

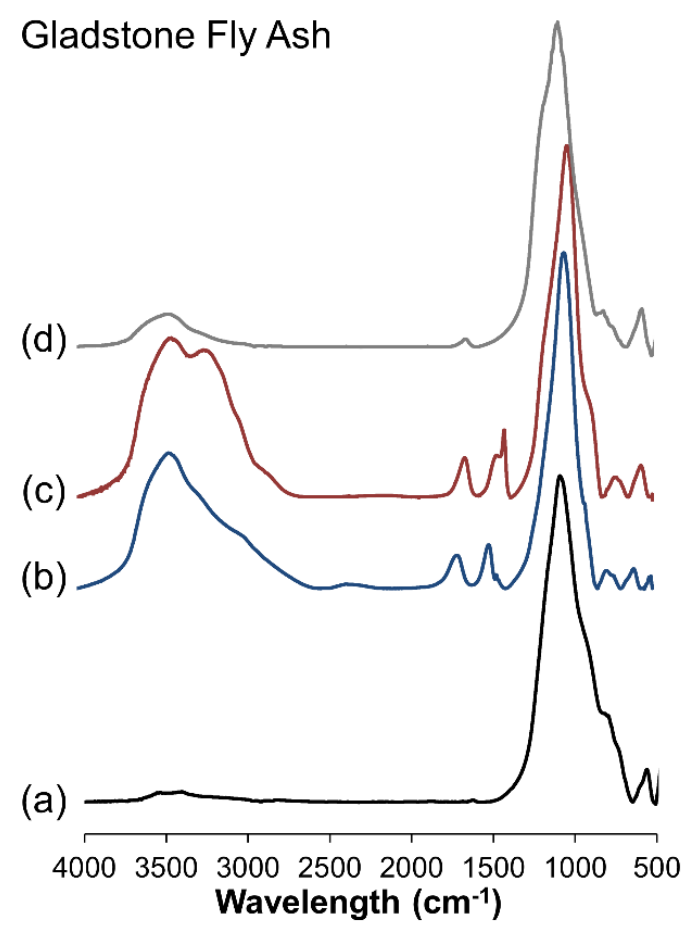

Figure 5.2 FTIR spectra of geopolymers prepared from different fly ashes. (a) raw fly ash, (b) fly ash-based geopolymer "as-synthesised", (c) $\mathrm{NH}_{4}{ }^{+}$-form of the geopolymer, (d) $\mathrm{NH}_{4}{ }^{+}$-geopolymer after heating to $550{ }^{\circ} \mathrm{C}$. 
After thermal treatment at $550{ }^{\circ} \mathrm{C}$ for 15 min (Figure 5.2d), the N-H stretching and bending modes have completely disappeared in all the geopolymers, confirming the total decomposition of $\mathrm{NH}_{4}{ }^{+}$and the formation of the $\mathrm{H}$-form of the geopolymer. Destruction of the silanol groups after the thermal treatment differs for the various fly ashes, with Gladstone showing severe destruction of the silanol groups, as deduced from the ratio of the hydroxyl band (in the range $3400-3700 \mathrm{~cm}^{-1}$ ) to the main peak at $\sim 1100 \mathrm{~cm}^{-1}$. However, catalysts prepared from the other fly ashes show a higher concentration of $\mathrm{OH}$ groups compared to the Gladstone and the clay-based geopolymer catalysts that were heated to $550{ }^{\circ} \mathrm{C}$ for $15 \mathrm{~min}$. (Figure 3.13). In addition, dealumination after thermal treatment is evident by the reappearance of the octahedral $\mathrm{Al}$ (EFAl) peak at $795 \mathrm{~cm}^{-1}$.

The effect of the ion exchange process on the fly ash-based geopolymers was studied by XRF analysis (Table 5.3). The main impact is the removal of at least $\sim 85 \%$ of the $\mathrm{Na}$ after ion exchange, with a corresponding slight alteration in the concentrations of the other oxides. The complete elemental analysis of these materials is attached in the Appendix A (Table A2).

Table 5.3 Metal oxides (wt.\%) of the as-synthesised and ion exchanged fly ash-based geopolymers ${ }^{\mathrm{a}}$.

\begin{tabular}{|c|c|c|c|c|c|c|c|}
\hline Sample & $\mathrm{SiO}_{2}$ & $\mathrm{Al}_{2} \mathrm{O}_{3}$ & $\mathrm{Fe}_{2} \mathrm{O}_{3}$ & $\mathrm{CaO}$ & $\mathrm{Na}_{2} \mathrm{O}$ & MgO & $\mathrm{K}_{2} \mathrm{O}$ \\
\hline Hyrock-Geo & 46.29 & 20.67 & 4.25 & 0.80 & 16.74 & 0.45 & 1.04 \\
\hline Geo-Hyrock-NH${ }_{4}^{+}$ & 54.08 & 24.70 & 4.53 & 0.86 & 4.16 & 0.46 & 0.22 \\
\hline Huntly Geo & 40.71 & 16.96 & 6.98 & 10.51 & 11.28 & 1.89 & 0.44 \\
\hline Geo-Huntly- $\mathrm{NH}_{4}{ }^{+}$ & 48.88 & 20.50 & 8.26 & 8.84 & 0.43 & 2.06 & 0.14 \\
\hline Geo-GS & 40.91 & 19.36 & 8.14 & 2.34 & 15.75 & 1.02 & 0.32 \\
\hline Geo-G.S.- $\mathrm{NH}_{4}^{+}$ & 48.90 & 23.27 & 9.05 & 2.54 & 1.20 & 1.12 & 0.10 \\
\hline Geo-Mt. Piper & 56.43 & 23.06 & 0.77 & 0.16 & 11.23 & 0.16 & 1.46 \\
\hline Geo-Mt. Piper- $\mathrm{NH}_{4}{ }^{+}$ & 61.01 & 25.34 & 0.84 & 0.17 & 0.60 & 0.16 & 0.92 \\
\hline
\end{tabular}

a determined by XRF 
SEM micrographs showing the morphology of all the fly ashes used in this study, and the corresponding geopolymers are presented in Figure 5.3. All the fly ashes display a typical spherical shape, and consist mainly of cenospheres with some solid spheres, and other amorphous debris. Fig. 5.3 shows the SEM micrograph of each geopolymer next to the corresponding parent fly ash. The synthesised geopolymers contain large amorphous particles with diameters $\sim 20 \mu \mathrm{m}$, composed of smaller particle aggregates forming voids within the larger particles, similar to the SEM micrographs obtained from clay-based geopolymers.

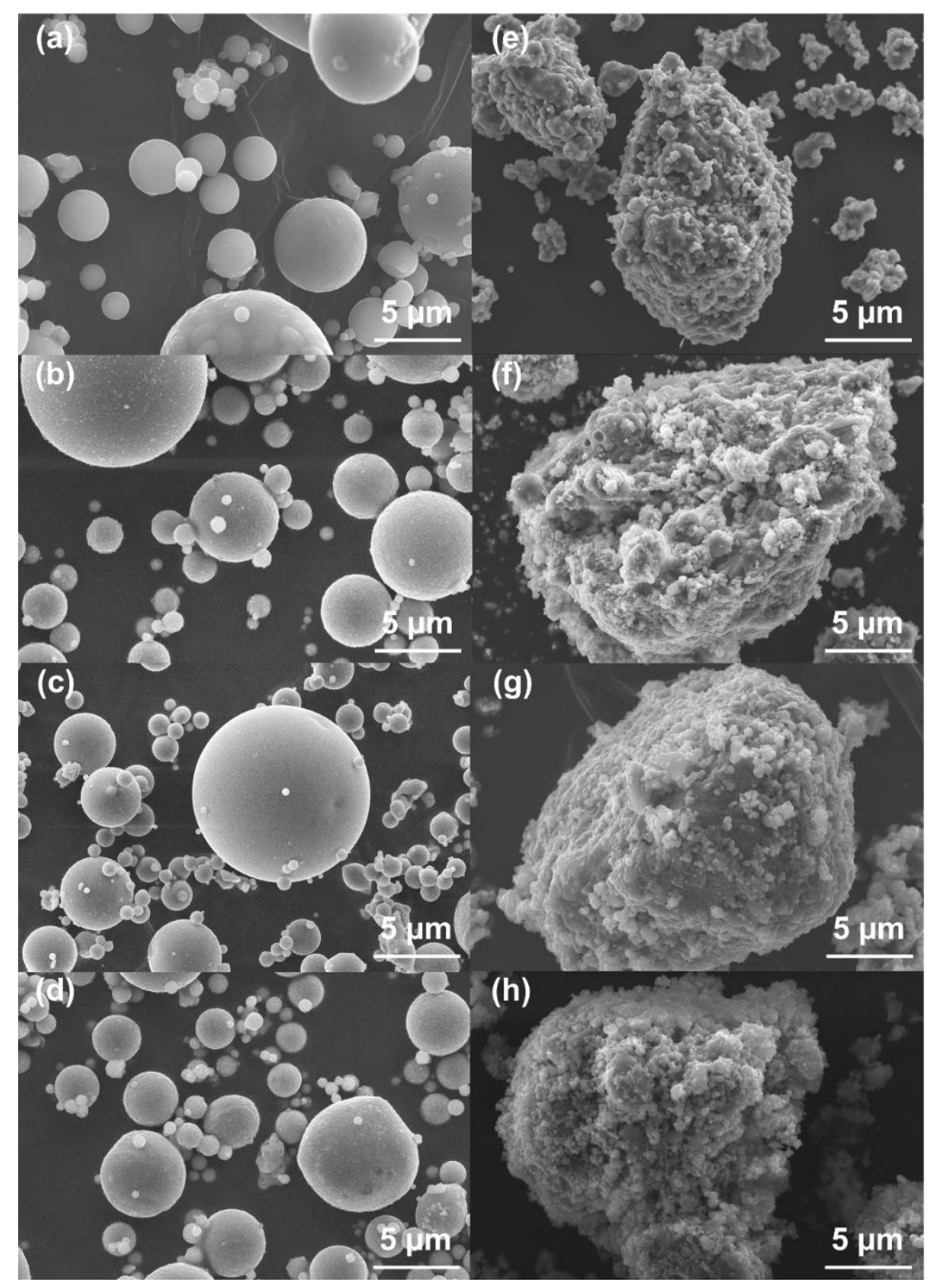

Figure 5.3 SEM micrographs the raw fly ashes (a-d) and the prepared geopolymers (eh). (a) Hyrock, (b) Huntly, (c) Mt. piper, (d) G.S., (e) Geo-Hyrock, (f) Geo-Huntly, (g) Geo-Mt. piper, (h) Geo-G.S. 
The average particle size distributions of the fly ashes and the synthesised fly ash-based catalysts are shown in Figure 5.4. All the raw fly ashes (Figure 5.4a) show a broad particle size distribution in the range $<1 \mu \mathrm{m}$ to $>500 \mu \mathrm{m}$, with an average particle size of $\sim 20 \mu \mathrm{m}$. The synthesised fly ash-based catalysts, on the other hand, show a smaller range of particle size distribution of $1-100 \mu \mathrm{m}$, with a similar average particle size distribution to that of the raw fly ash; this is especially the case for the Geo-Hyrock and Geo-Huntly fly ashes whereas Geo-G.S. and Geo-Mt. piper show a smaller average size $(\sim 15 \mu \mathrm{m}$, Table 5.4).

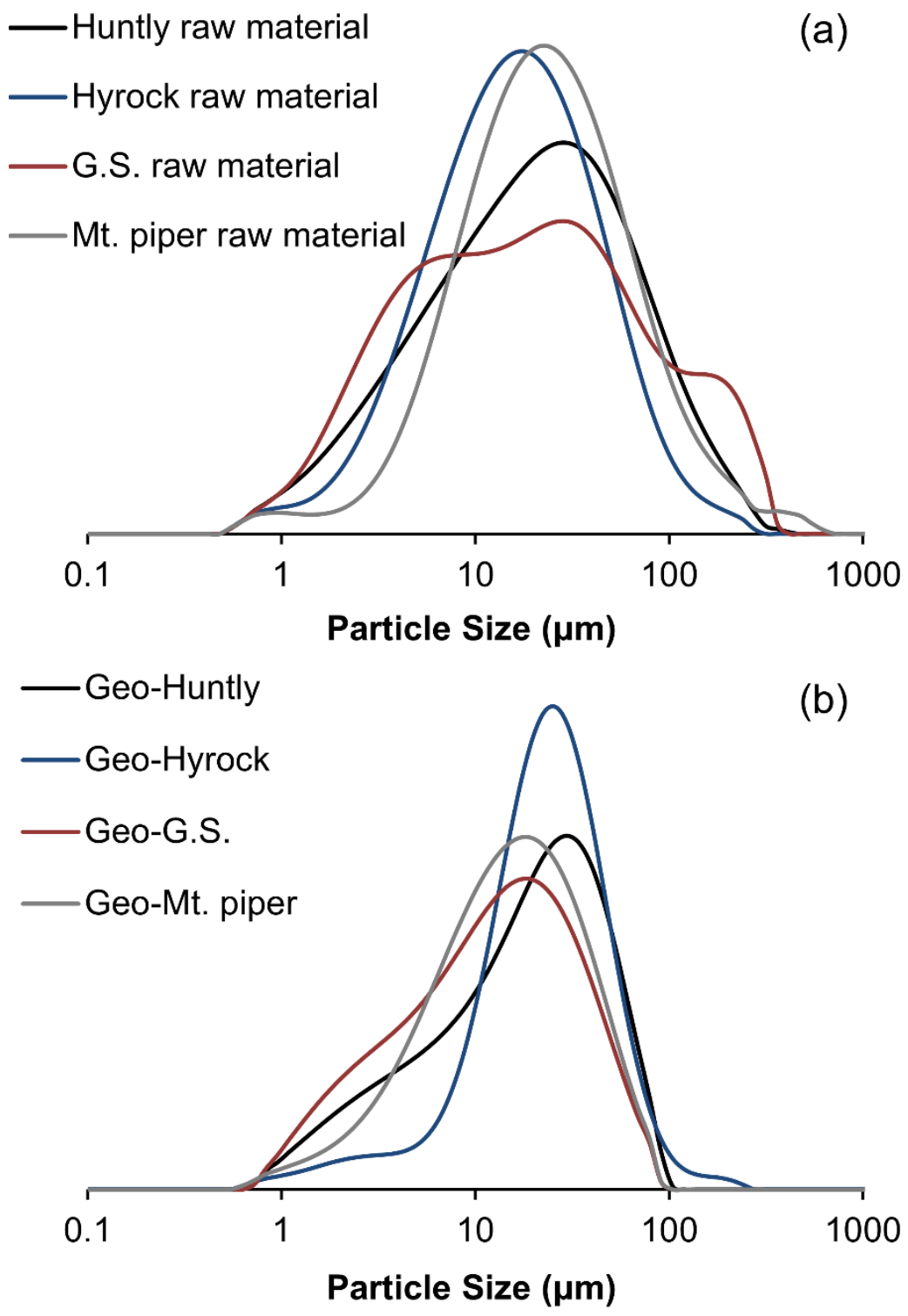

Figure 5.4 Particle size distribution of the raw fly ashes (a) and the corresponding fly ash-based catalysts (b). 
Table 5.4 Physical and chemical properties of the fly ash-based catalysts.

\begin{tabular}{ccccc}
\hline Catalyst & $\begin{array}{c}\text { Acid amount } \\
(\mathbf{m m o l} / \mathbf{g})^{a}\end{array}$ & $\begin{array}{c}\text { Average } \\
\text { particle size } \\
(\boldsymbol{\mu m})\end{array}$ & $\begin{array}{c}\boldsymbol{S}_{\text {BET }} \\
\left(\mathbf{m}^{\mathbf{2}} / \mathbf{g}\right)\end{array}$ & $\begin{array}{c}\boldsymbol{V}_{\text {total }} \\
\left(\mathbf{c m}^{\mathbf{3}} / \mathbf{g}\right)\end{array}$ \\
\hline Geo-Hyrock & 0.33 & 25 & 144 & ---- \\
Geo-Huntly & 0.39 & 20 & 59 & 0.09 \\
Geo-Mt. piper & 0.25 & 16 & 73 & 0.02 \\
Geo-G.S. & 0.13 & 14 & ---- & 0.04 \\
\hline
\end{tabular}

a determined from the TGA profile of pyridine desorption.

${ }^{\mathrm{b}}$ single point at $p / p^{o}=0.985$.

The surface areas and total pore volumes of the catalysts are summarised in table 5.4 and a representative $\mathrm{N}_{2}$ isotherm of Geo-Huntly is shown in Figure 5.5. The GeoHyrock catalyst possesses a high surface area $\left(\sim 140 \mathrm{~m}^{2} / \mathrm{g}\right)$ while the other catalysts have values closer to those of most of the clay-based geopolymer catalysts (Table 3.1).

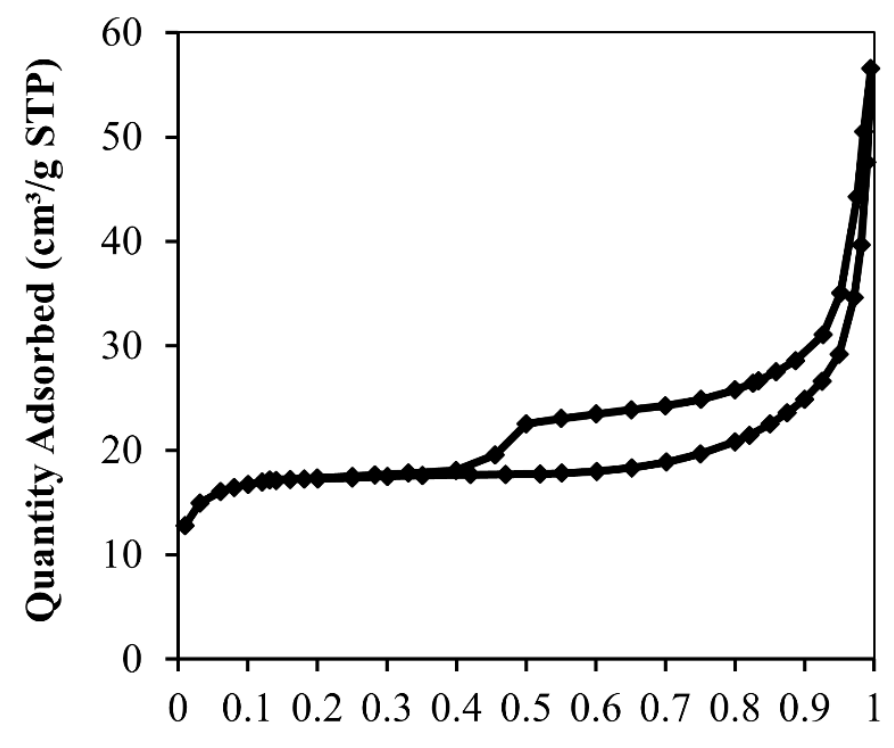

\section{Relative Pressure $\left(\mathbf{p} / \mathbf{p}^{\circ}\right)$}

Figure 5.5 $\mathrm{N}_{2}$ adsorption-desorption isotherms of Geo-Huntly catalyst.

The very small pore volume of the fly ash-based catalysts, also shown in Table 5.4, can be ascribed to the impurities present in the raw fly ash, resulting in the pores of the resulting catalysts being filled with amorphous debris, crystalline phases and metal 
oxides. This may have been the reason for the distorted $\mathrm{N}_{2}$-isotherms for some of these catalysts (the missing data in Table 5.4).

The pore sizes of the catalysts and their morphology was investigated further by TEM (Figure 5.6). All the catalysts show mesoporosity, with pore diameters varying from $5 \mathrm{~nm}$ for Geo-Mt. piper (Figure 5.6c) up to $\sim 30 \mathrm{~nm}$ for Geo-Hyrock (Figure 5.6a). In addition, macroporosity is also observed in some of the catalysts (e.g. Geo-G.S., Figure $5.6 \mathrm{~d})$.

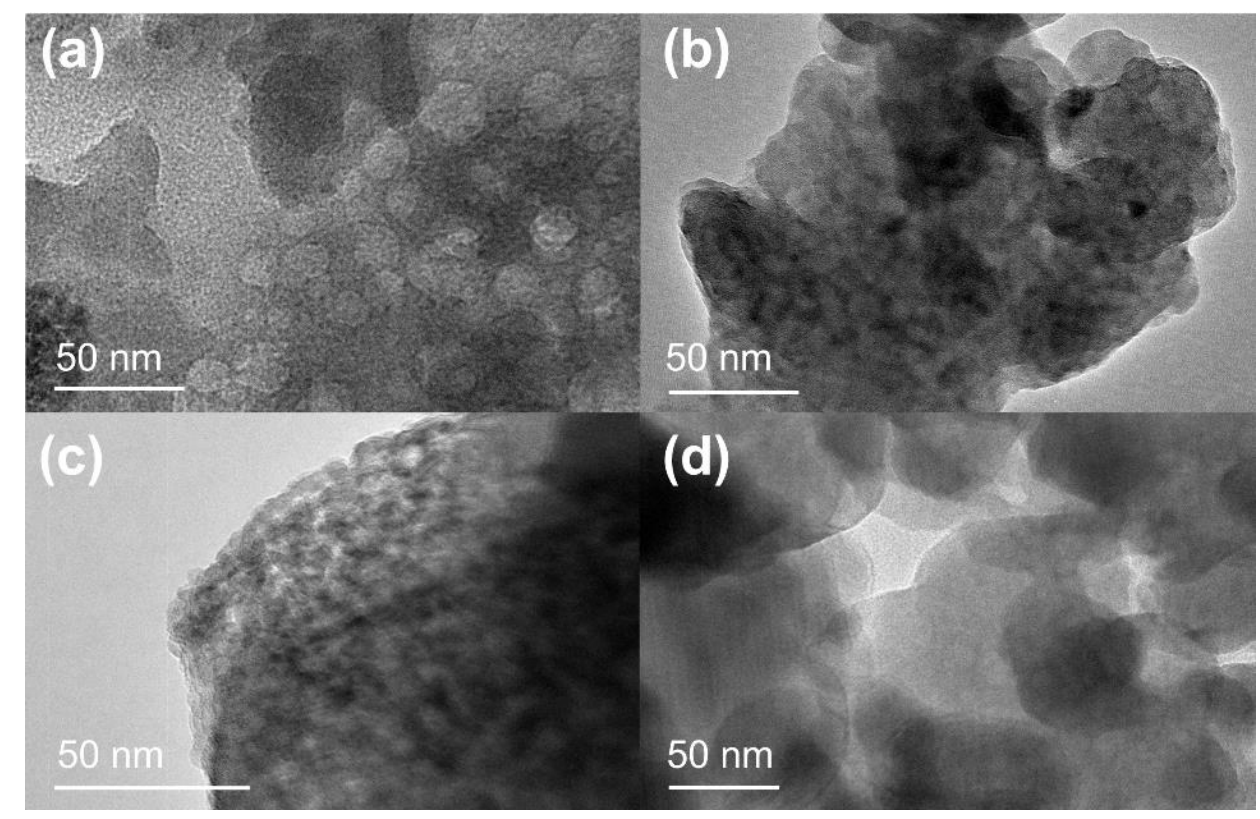

Figure 5.6 TEM micrographs of the fly ash-based geopolymer catalysts. (a) GeoHyrock, (b) Geo-Huntly, (c) Geo-Mt. piper, (d) Geo-G.S.

The TGA profiles of the pyridine desorbed from the activated fly ash-based catalysts are shown in Figure 5.7 and the corresponding acidities in Table 5.4. The largest amount of desorbed pyridine was from the Geo-Huntly (Figure 5.7b) in which $\sim 3$ wt.\% of pyridine was desorbed in the temperature range $\sim 50$ to $\sim 400{ }^{\circ} \mathrm{C}$. Geo-Hyrock (Figure 5.7a) and Geo-Mt. piper (Figure 5.7c) showed $\sim 2.6$ and $~ 2.0 \mathrm{wt} . \%$ of desorbed pyridine respectively in the temperature range $100-350{ }^{\circ} \mathrm{C}$. On the other hand, Geo-G.S. showed the lowest amount of desorbed pyridine, with only $~ 1 \mathrm{wt}$ \% of pyridine being desorbed in the temperature range 50 to $350{ }^{\circ} \mathrm{C}$ (Figure $5.7 \mathrm{~d}$ ). 

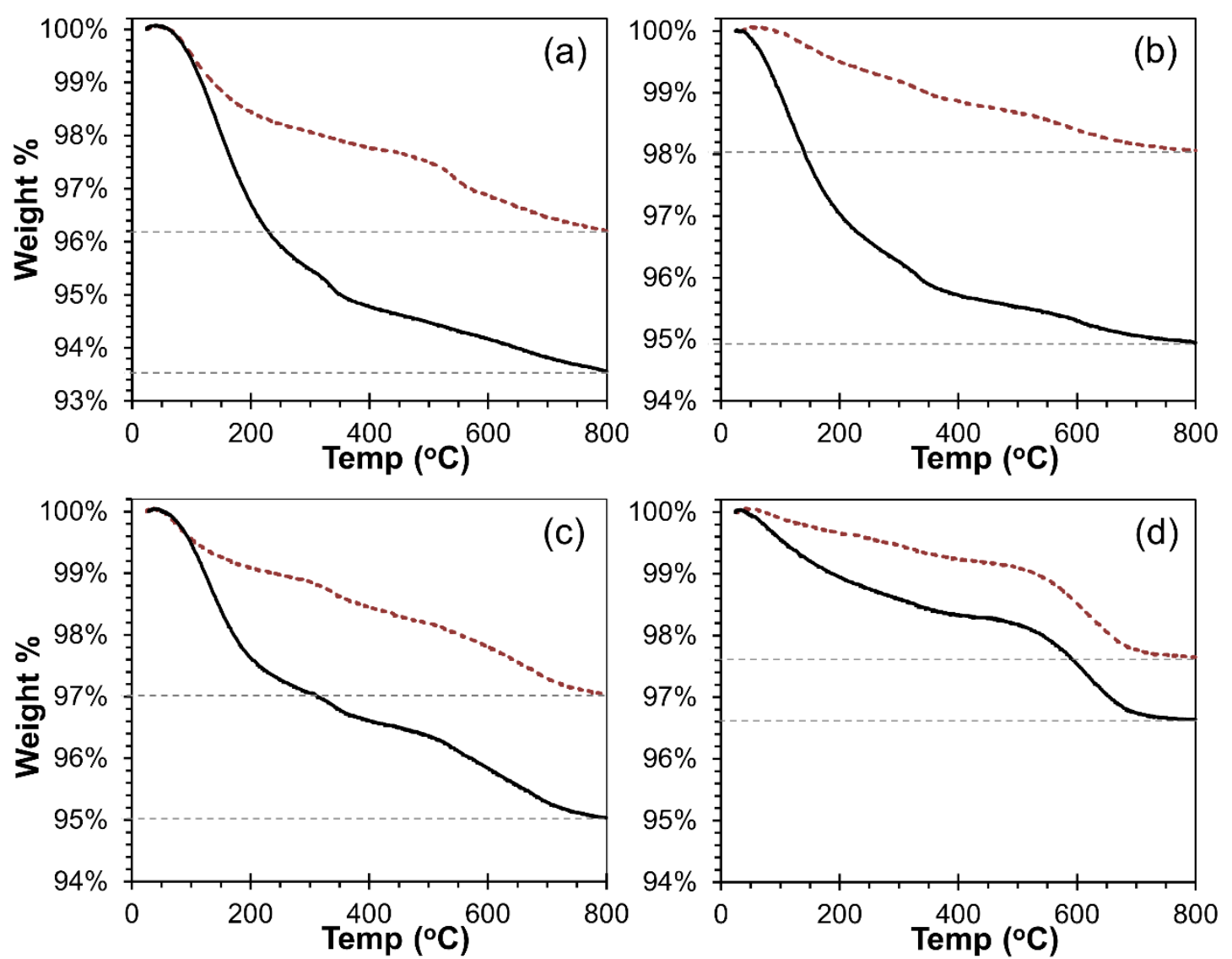

Figure 5.7 TGA profiles of pyridine desorption from the fly ash-based catalysts. The dashed line is the background and the solid line represents the pyridine desorption. (a) Geo-Hyrock, (b) Geo-Huntly, (c) Geo-Mt. piper, (d) Geo-G.S.

These results are consistent with the FTIR spectra for adsorbed pyridine (Figure 5.8) where the highest intensity of the peaks was observed in Geo-Huntly (Figure 5.8b), followed by Geo-Hyrock and Geo-Mt. piper, whereas in Geo-G.S. these peaks were hardly detected. As discussed in Section 2.2.6.2, the bands at 1445, 1600 and $1620 \mathrm{~cm}^{-}$ 1 are associated with Lewis acidic sites while the band at $\sim 1545 \mathrm{~cm}^{-1}$ represents Bronsted acidic sites, with a combination of both sites represented by the peak at 1490 $\mathrm{cm}^{-1}$. 

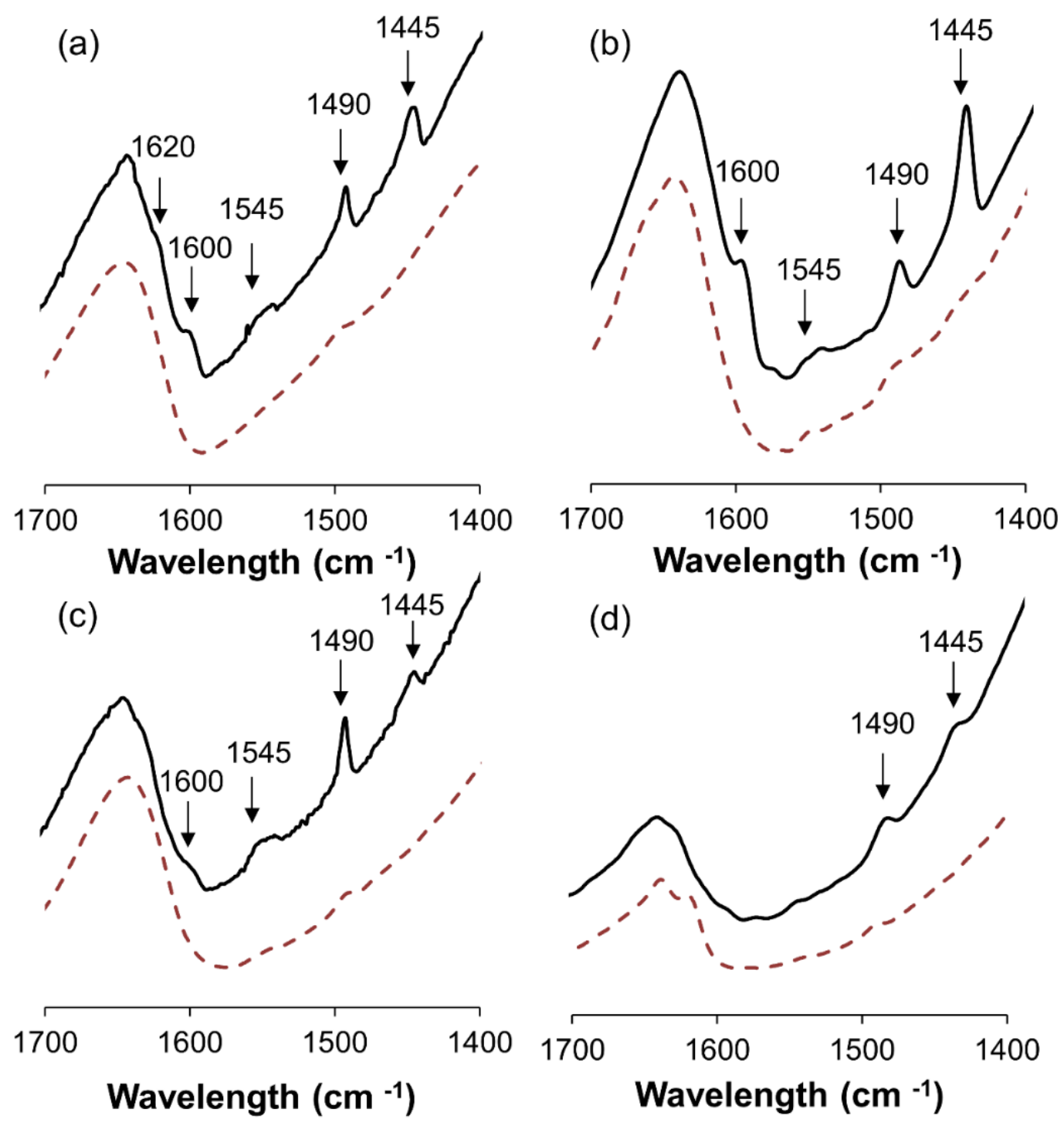

Figure 5.8 FTIR spectra of the fly ash-based catalysts without pyridine (dashed line), and with desorbed pyridine (solid line). (a) Geo-Hyrock, (b) Geo-Huntly, (c) Geo-Mt. piper, (d) Geo-G.S.

Qualitative elemental analysis of the prepared catalysts was carried out using EDS in conjunction with SEM to investigate the distribution of the metal oxides present in the geopolymer particles and the manner in which these oxides are attached to the geopolymer particles. Figure 5.9 shows a representative EDS analysis of the GeoHyrock catalyst over the area shown in the SEM image insert. 


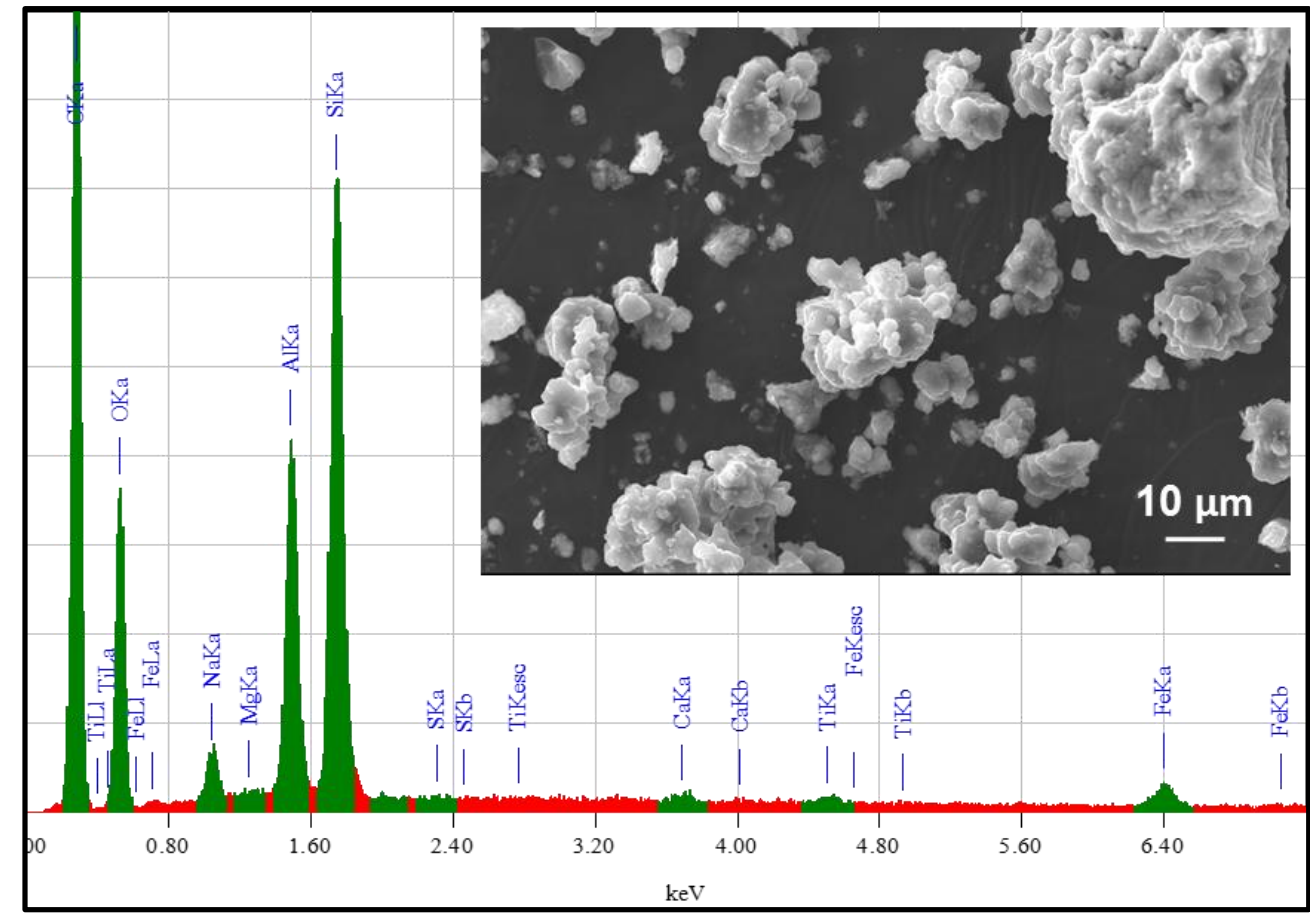

Figure 5.9 EDS analysis of Geo-Hyrock catalyst.

The main elements present in the solid are $\mathrm{Si}, \mathrm{Al}, \mathrm{O}, \mathrm{Na}, \mathrm{Fe}$ with traces of $\mathrm{Mg}$, and $\mathrm{Ca}$. The distribution of these elements, obtained by EDS mapping, is presented in Figure 5.10. The maps below show that the densest images are from $\mathrm{Si}$ and $\mathrm{Al}$, the most abundant elements in the sample, followed by $\mathrm{Na}$ and $\mathrm{Fe}$, with $\mathrm{Mg}$ and $\mathrm{Ca}$ providing the least dense images. The bright spots detected in the Fe map (Figure 5.10e) were analysed at higher magnification (Figure 5.11). 


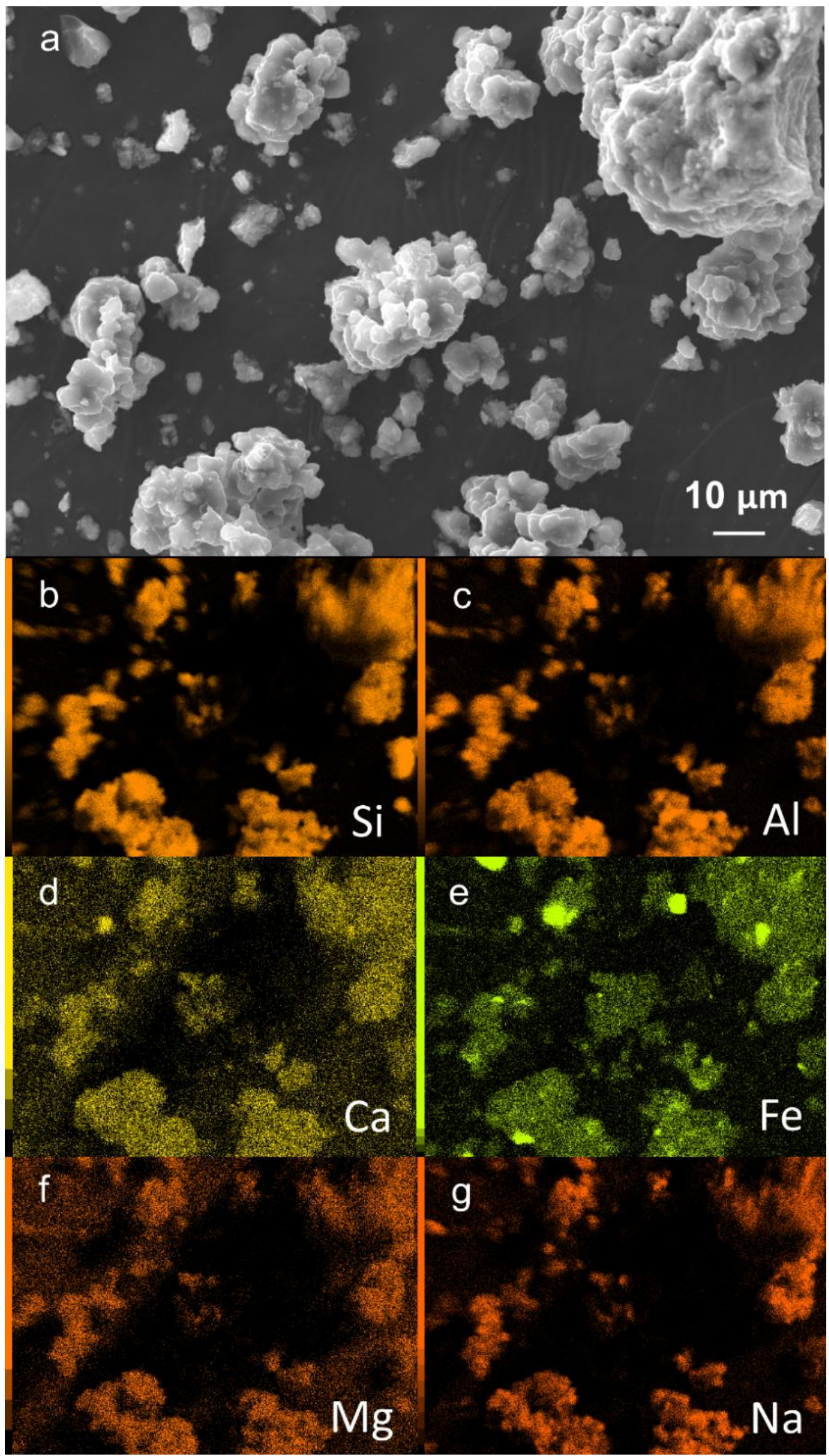

Figure 5.10 Element distribution maps of Geo-Hyrock catalyst. 
High magnification SEM images of some of the bright Fe spots (Figure 5.11a-d), circled in red in the two source images at the top, show that the Fe species (most probably $\mathrm{Fe}_{2} \mathrm{O}_{3}$ ) present in these areas are actually part of the geopolymer particles, being inserted within the geopolymer particles. This is very important and highly desirable feature for catalysis applications, where Fe species are expected to act as the active sites, providing a high degree of reusability and long catalyst lifetime.

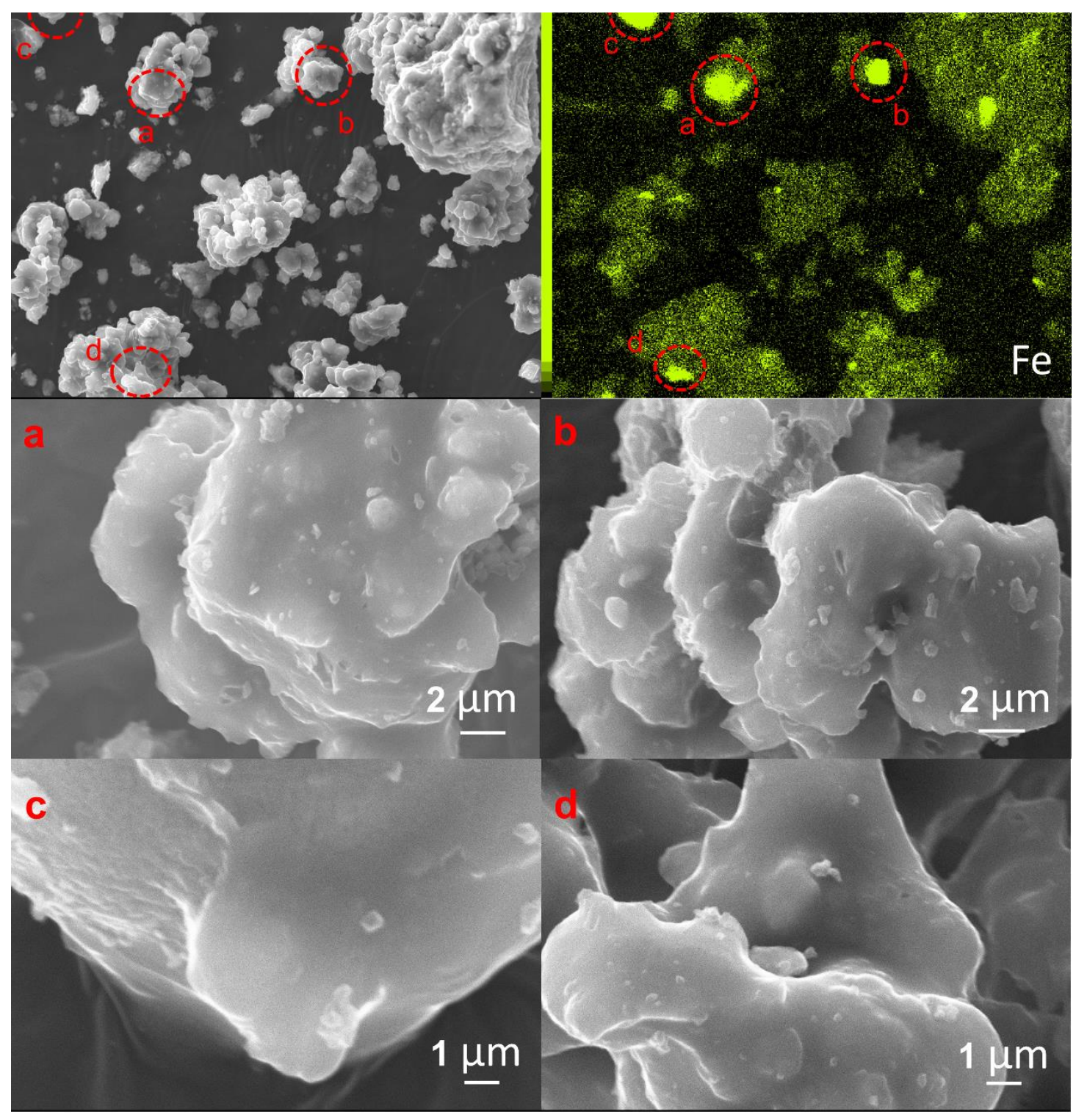

Figure 5.11 High magnification SEM images of specific spots containing Fe species. Each image (a-d) represents the red circled spots identified at the top two source images. 


\subsubsection{Catalytic reactivity}

The catalytic reactivity of the fly ash-based catalysts in the alkylation of benzene and substituted aromatics with $\mathrm{BzCl}$ as alkylating agent was initially investigated over the Geo-Hyrock catalyst (Figure 5.12). Very high reactivity was achieved for substituted benzenes, particularly anisole, p-xylene and mesitylene in which the alkylation was almost complete within $1 \mathrm{hr}$ (Figure 5.12a) with high selectivity towards the monobenzylated products (>95\%) (Figure 5.12b).
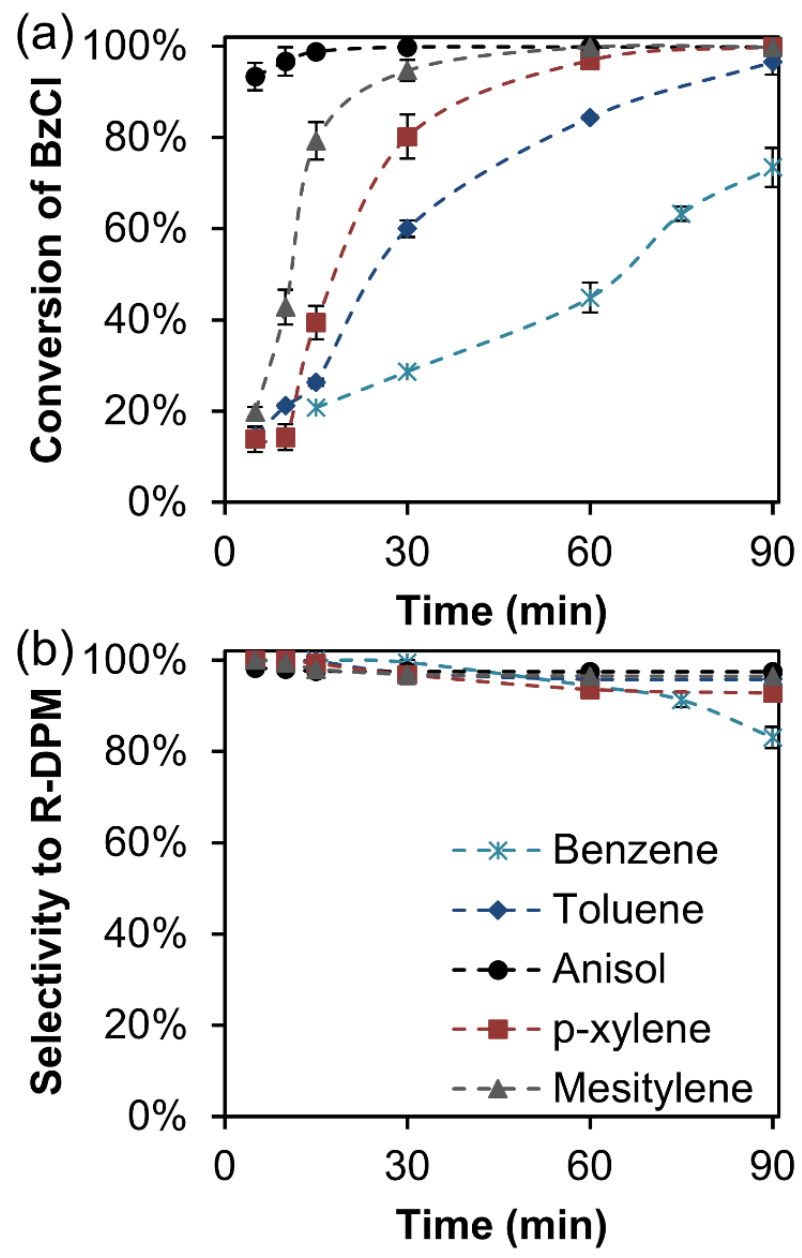

Figure 5.12 Catalytic reactivity of Geo-Hyrock in alkylation of several aromatics. (a) conversion of $\mathrm{BzCl}$, (b) selectivity to monobenzylated products. Reaction conditions; $13.0 \mathrm{ml}$ of each aromatic; $1.0 \mathrm{ml} \mathrm{BzCl} ; 0.1 \mathrm{~g}$ catalyst; $\mathrm{T}=80{ }^{\circ} \mathrm{C}$.

Lower reactivity was observed for the alkylation of toluene and benzene, with $~ 80$ and $40 \%$ conversion of $\mathrm{BzCl}$ respectively being achieved within the first hour. Nonetheless, after 90 min. of reaction, $>96 \%$ conversion of $\mathrm{BzCl}$ was achieved with high selectivity 
$(>95 \%)$ in the alkylation of toluene, and up to $75 \%$ conversion in the alkylation of benzene with a selectivity of $\sim 85 \%$ to DPM.

The raw Hyrock fly ash shows poor reactivities under identical reaction conditions (Figure 5.13a), being completely inactive for the alkylation of benzene and toluene, and producing only $\sim 10 \%$ conversion of $\mathrm{BzCl}$ in the alkylation of $\mathrm{p}$-xylene. However, higher activity was observed for the alkylation of anisole and mesitylene, with $\mathrm{BzCl}$ conversion of $\sim 70$ and $40 \%$ respectively.
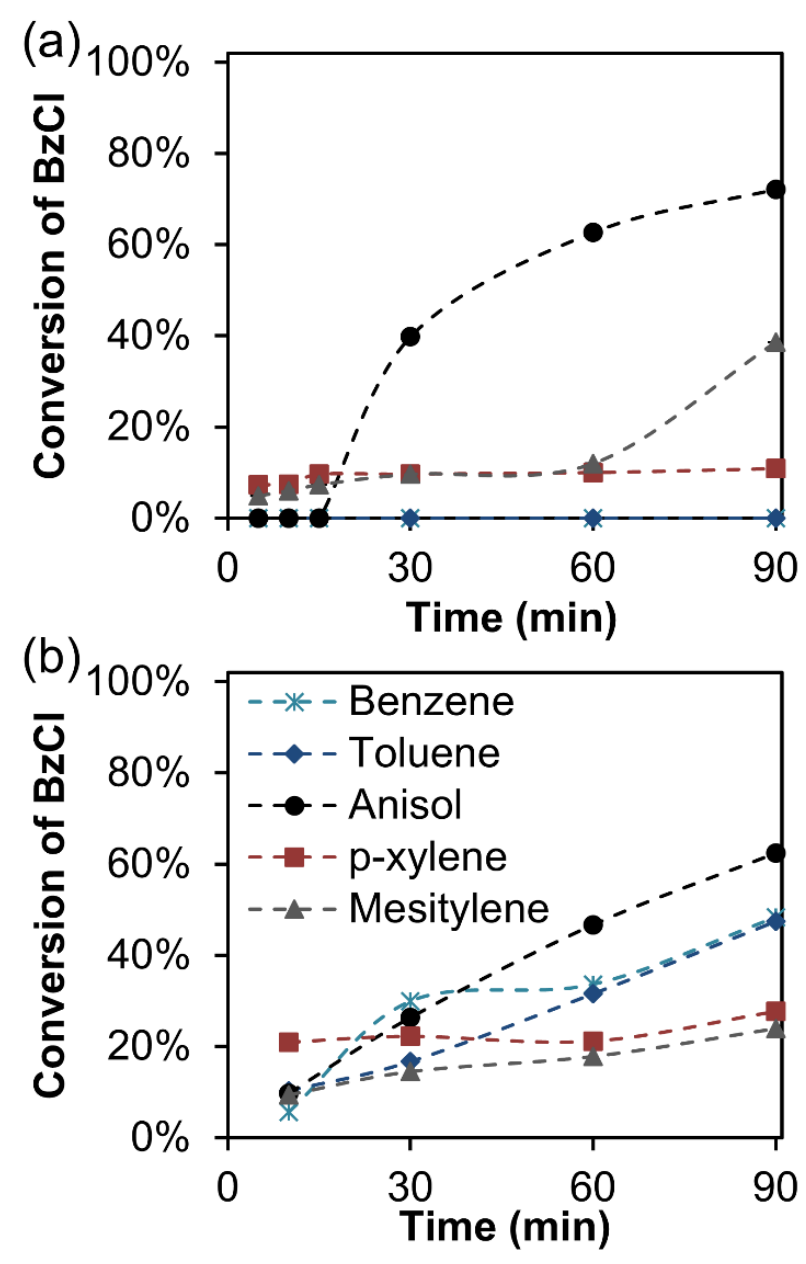

Figure 5.13 Catalytic reactivity of raw Hyrock fly ash (a) and H-Y zeolite (b) in the alkylation of various aromatics with $\mathrm{BzCl}$. Reaction conditions; $13.0 \mathrm{ml}$ of each aromatic; $1.0 \mathrm{ml} \mathrm{BzCl} ; 0.1 \mathrm{~g}$ catalyst; $\mathrm{T}=80^{\circ} \mathrm{C}$.

Similar results were also obtained with H-Y zeolite as the catalyst (Figure 5.13b) in which the highest reactivity was obtained for the alkylation of anisole $(\sim 60 \%$ 
conversion of $\mathrm{BzCl}$ at $90 \mathrm{~min})$, followed by toluene and benzene ( $45 \%$ and $\sim 20 \%$ conversion of $\mathrm{BzCl}$ in the alkylation of $\mathrm{p}$-xylene and mesitylene respectively).

It is interesting to note that the reactivity of the Geo-Hyrock catalyst (Figure 5.12a) and the raw Hyrock fly ash (Figure 5.13a) in the alkylation of these aromatics was in the order of anisole> mesitylene> p-xylene> toluene> benzene; this order of reactivity suggests a dependence mainly on the electron density of the aromatic ring, with the size of the reactant or product having little or no impact. The influence of the molecular size of the reactants and products on the catalytic reactivity is most apparent in the case of H-Y zeolite (Figure 5.13b) where the lowest reactivity is obtained for the alkylation of mesitylene, which is ascribed to its restricting micropores. A similar effect was observed for the clay-based catalysts (Figure 4.13) in which the catalytic reactivity in alkylation of mesitylene was slightly less than that of $\mathrm{p}$-xylene.

The absence of the confinement effect when fly ash-based catalysts are used suggests that the reaction most probably occurs on the surface of the catalyst, since the raw fly ash is a nonporous material and the fly ash-based geopolymers have very small pore sizes due to their being blocked with other oxides and amorphous debris (Table 5.4).

The reaction kinetics of the alkylation of all the present aromatics over Geo-Hyrock was modelled using the Langmuir-Hinshelwood pseudo-first-order kinetic model (Figure 5.14).

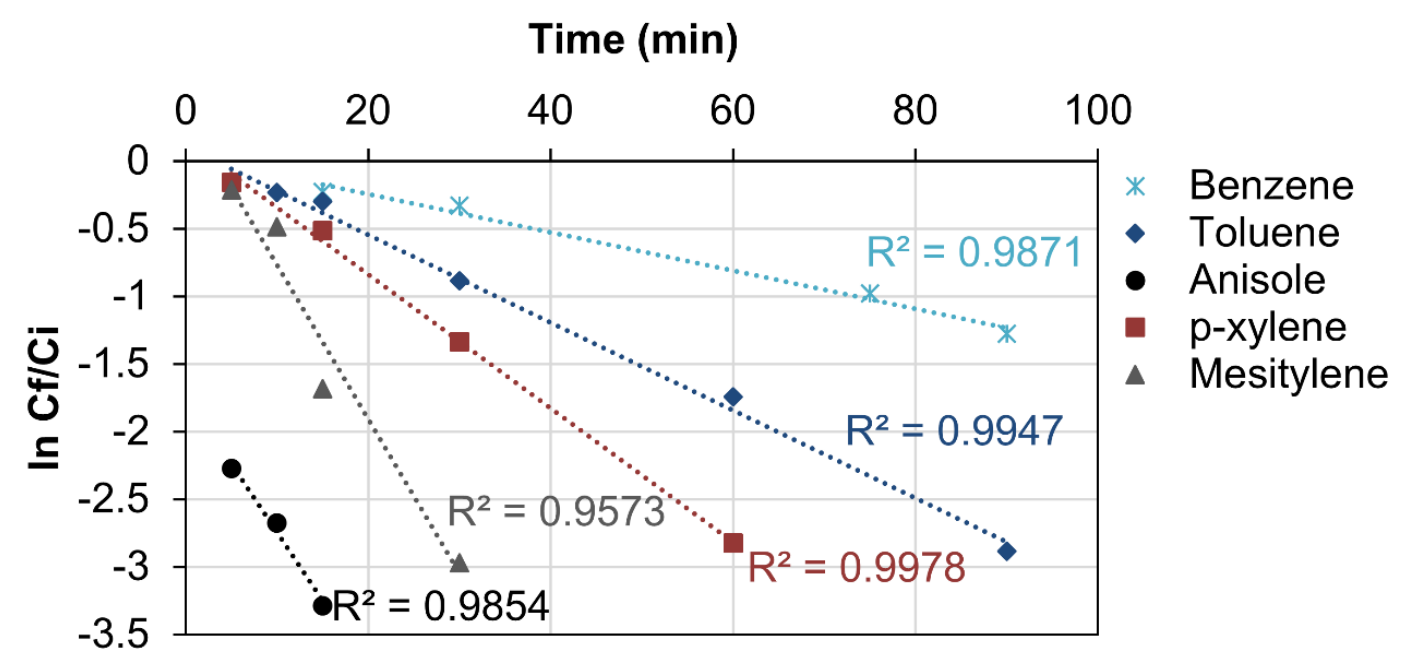

Figure 5.14 Langmuir-Hinshelwood pseudo-first-order kinetic model for the alkylation reactions of benzene, toluene, anisole, p-xylene, and mesitylene over the Geo-Hyrock catalyst. 
Figure 5.14 shows that the obtained experimental data are fitted well by the suggested model, as expected since a first step in heterogeneous catalytic reactions is the adsorption process, which plays a major role in heterogeneous catalytic reactions ${ }^{[181]}$. The slopes of the lines in Figure 5.14 were used to determine the reaction constants of the alkylation reactions of the aromatics, as shown in Table 5.5.

Table 5.5 Kinetic parameters of the catalytic reactivity of Geo-Hyrock in the alkylation of the various aromatics with $\mathrm{BzCl}$ after 30 min. reaction time ${ }^{\mathrm{a}}$.

\begin{tabular}{lcccc}
\hline Substituent & $\begin{array}{c}\text { Conversion } \\
(\boldsymbol{\%})\end{array}$ & $\begin{array}{c}\text { Selectivity } \\
(\boldsymbol{\%})\end{array}$ & $\begin{array}{c}\text { Specific reaction rate } \\
\left(\mathbf{m m o l}_{\left.\mathbf{B z C l} \cdot \mathbf{g}_{\mathbf{c a t}} \mathbf{- 1} \cdot \mathbf{m i n}^{-\mathbf{1}}\right)}\right.\end{array}$ & $\mathbf{k}\left(\mathbf{x} \mathbf{1 0} \mathbf{~ m i n}^{\mathbf{- 1}}\right)$ \\
\hline Benzene & 29 & 100 & 0.82 & 14.1 \\
Toluene & 60 & 100 & 1.70 & 32.4 \\
Anisole & 100 & 98 & 2.83 & 101.3 \\
p-xylene & 80 & 97 & 2.14 & 49.4 \\
Mesitylene & 95 & 97 & 2.75 & 114.3 \\
\hline
\end{tabular}

${ }^{\text {a }}$ Reaction conditions; $13.0 \mathrm{ml}$ of each aromatic; $1.0 \mathrm{ml} \mathrm{BzCl} ; 0.1 \mathrm{~g}$ catalyst; $\mathrm{T}=80{ }^{\circ} \mathrm{C}$, $\mathrm{t}=30 \mathrm{~min}$.

As discussed above, fly ashes collected from different sources possess differing chemical and physical properties which are expected to affect their performance in applications such as catalysis. For this reason, three further series of geopolymer catalysts were synthesised from different fly ashes from other sources, and their catalytic reactivities were investigated in the alkylation of the same series of aromatics (Figure 5.15).

Both the Geo-Huntly and Geo-G.S. fly ash-based catalysts show high catalytic reactivities in the alkylation of all the substituted aromatics (Figures 5.15a and 5.15c respectively); however the latter shows no activity towards the alkylation of benzene. The alkylation reactions were almost complete within 1 hour, similar to the Geo-Hyrock catalyst, but during the first 30 minutes of reaction, Geo-Huntly and Geo-G.S. show higher reactivity, particularly in the alkylation of mesitylene, xylene, and toluene. The selectivity towards monobenzylated products was usually $>95 \%$ in the alkylation of all 
the aromatics except for the alkylation of benzene, where the selectivity decreases at high conversion\% to $\sim 85 \%$, as found in the Geo-Hyrock catalyst. On the other hand the Geo-Mt. Piper catalyst (Figure 5.15e) is completely inactive in the alkylation of benzene, toluene and p-xylene, with very poor reactivity towards the alkylation of anisole and mesitylene ( $<20 \%$ conversion).
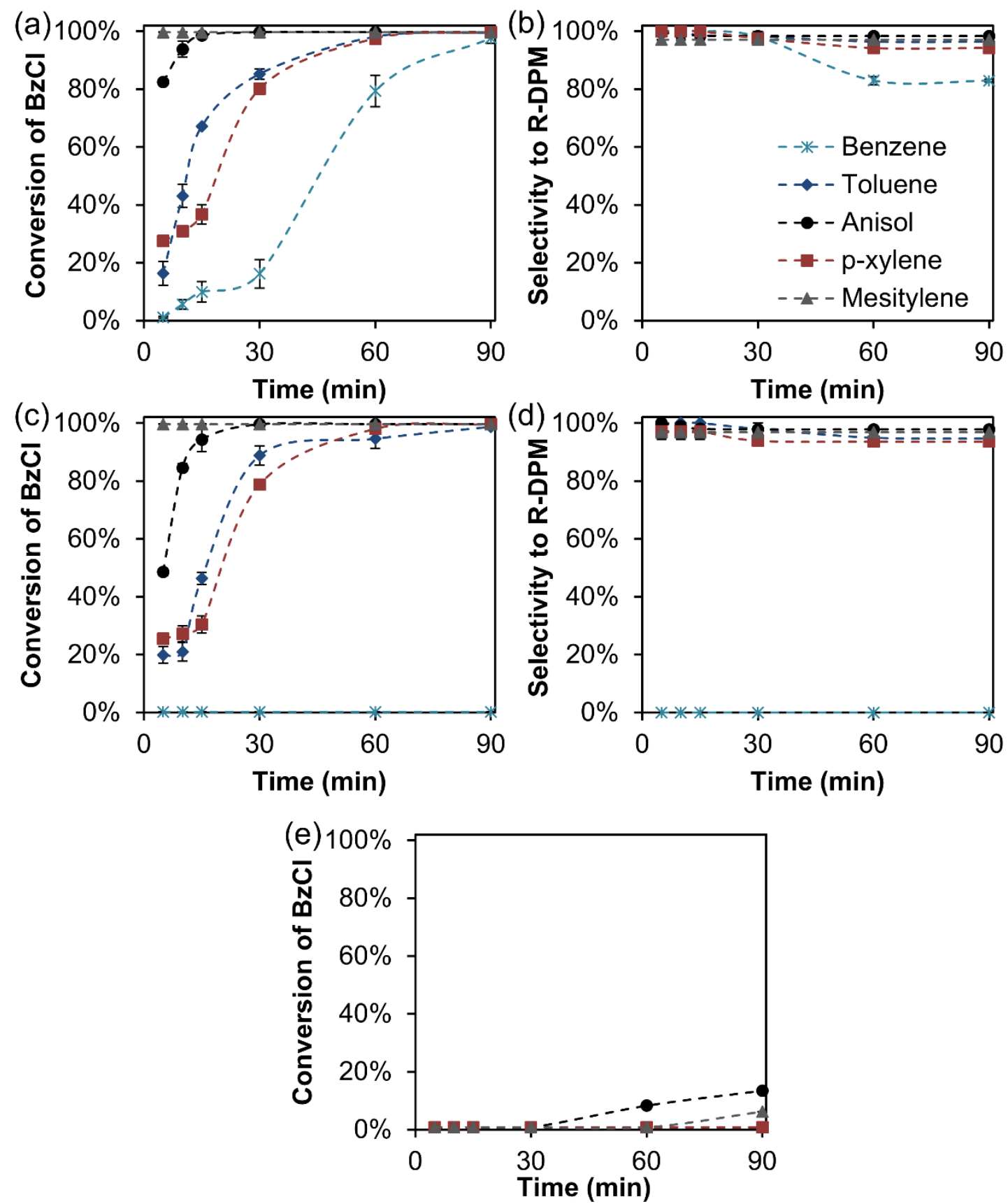

Figure 5.15 Catalytic reactivities of various fly ash-based catalysts in the alkylation of several aromatics. (a) and (b) Geo-Huntly, (c) and (d) Geo-G.S., (e) Geo-Mt. Piper. Reaction conditions; $13.0 \mathrm{ml}$ of each aromatic; $1.0 \mathrm{ml} \mathrm{BzCl} ; 0.1 \mathrm{~g}$ catalyst; $\mathrm{T}=80{ }^{\circ} \mathrm{C}$. 
Table 5.6 compares the catalytic performance of the fly ash-based catalysts, H-Y zeolite and raw Hyrock fly ash in the alkylation of toluene with $\mathrm{BzCl}$ at $30 \mathrm{~min}$. reaction time. Figure 5.15 and Table 5.6 shows that Geo-G.S. showed the greatest reactivity despite barely containing any acidic sites, as shown by the pyridine adsorption experiments (Figure5.8d). The Geo-Mt. Piper catalyst, on the other hand, was completely inactive, although it contains both Bronsted and Lewis acid sites (Figure 5.8c). These results suggest that the active sites for this reaction and the alkylation of the substituted aromatics arise from the $\mathrm{Fe}_{2} \mathrm{O}_{3}$ content of the fly ash-based catalysts, and explains the high reactivity of the Geo-G.S. catalyst (9.05 wt.\% $\mathrm{Fe}_{2} \mathrm{O}_{3}$ ), whereas Geo-Mt. Piper (0.84 wt.\% $\left.\mathrm{Fe}_{2} \mathrm{O}_{3}\right)$ is inactive.

Table 5.6 Comparison of the catalytic reactivity of the various catalysts in the alkylation of toluene with $\mathrm{BzCl}$ at 30 min. reaction time ${ }^{\text {a }}$.

\begin{tabular}{cccccc}
\hline Catalyst & $\begin{array}{c}\text { Conversion } \\
\text { \% }\end{array}$ & $\begin{array}{c}\text { Selectivity }^{\mathbf{b}} \\
\mathbf{\%}\end{array}$ & $\begin{array}{c}\text { Specific } \\
\text { reaction rate }^{\mathbf{c}}\end{array}$ & TON $^{\text {TOF }}$ & $\begin{array}{c}\text { TOF } \\
\left(\mathbf{s}^{-\mathbf{1}}\right)\end{array}$ \\
\hline Geo-Hyrock & 60 & 97 & 1.70 & 180 & 0.10 \\
Geo-Huntly & 85 & 98 & 2.47 & 143 & 0.08 \\
Geo-G.S. & 89 & 98 & 2.57 & 136 & 0.08 \\
Geo-Mt. piper & 0 & 0 & 0.00 & 0 & 0.00 \\
$\begin{array}{c}\text { H-Y zeolite } \\
\text { Hyrock-raw }\end{array}$ & 17 & 99 & 0.48 & 8 & 0.00 \\
material & 0 & 0 & 0.00 & 0 & 0.00 \\
\hline
\end{tabular}

${ }^{\mathrm{a}}$ reaction conditions; $0.1 \mathrm{~g}$ cat; $1 \mathrm{ml} \mathrm{BzCl} ; 13 \mathrm{ml}$ toluene; $\mathrm{T}=80^{\circ} \mathrm{C} ; \mathrm{t}=30 \mathrm{~min}$.

${ }^{\mathrm{b}}$ selectivity to monobenzylated products.

${ }^{\mathrm{c}}\left(\mathrm{mmol}_{\mathrm{BzCl}} \cdot \mathrm{g}_{\mathrm{cat}}{ }^{-1} \cdot \mathrm{min}^{-1}\right)$.

It is possible that the inactivity of Geo-G.S. in the alkylation of benzene may suggest that in this particular reaction the active sites could be a combination of both Lewis and Bronsted sites arising from the aluminosilicate geopolymer framework and the $\mathrm{Fe}_{2} \mathrm{O}_{3}$ present from the original fly ash. This would explain why Geo-Hyrock and Geo-Huntly, with a combination of Lewis, Bronsted, and $\mathrm{Fe}_{2} \mathrm{O}_{3}$ sites, are the only active catalysts for the alkylation of benzene. 
The TON and TOF values for the alkylation of toluene by the various fly ash-based catalysts were calculated (Table 5.6) according to the amount of $\mathrm{Fe}_{2} \mathrm{O}_{3}$ present in each catalyst as determined by XRF (Table 5.3). Although not all the $\mathrm{Fe}_{2} \mathrm{O}_{3}$ may be exposed to the reactants, (i.e. accessible to the reactant, thereby allowing it to undergo reaction), if the total $\mathrm{Fe}_{2} \mathrm{O}_{3}$ content is used to determine the TON values, this would result in a lower limit to these values, which is beneficial for comparison purposes and would provide an approximate estimate of the catalysts' reactivities.

The reactivity of $\mathrm{Fe}_{2} \mathrm{O}_{3}$ in the Friedel-Crafts alkylation reaction over iron-containing catalysts (Fe-ZSM-5 zeolite ${ }^{[181]}$ and an iron-containing mesoporous aluminophosphate molecular sieve, $\mathrm{Fe}-\mathrm{AlPO}_{4}{ }^{[150 \mathrm{c}]}$ ) has been reported and a redox mechanism proposed for this reaction (Figure 5.16).

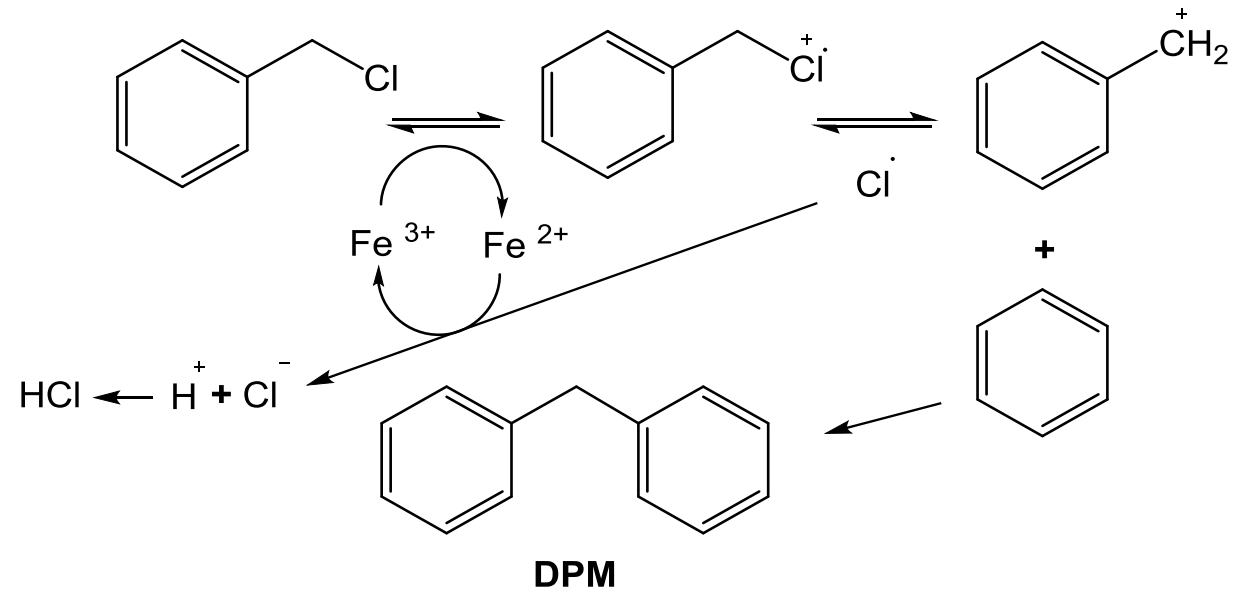

Figure 5.16 Proposed redox mechanism of Fe-containing catalysts in alkylation of benzene with $\mathrm{BzCl}$ (redrawn from ref. ${ }^{[181]}$ ).

Table 5.7 compares the catalytic reactivities of some of the present fly ash-based catalysts with other Fe-containing solid catalysts in the alkylation of benzene with $\mathrm{BzCl}$, under identical reaction conditions, unless otherwise stated. The data indicate that the present fly ash-based catalysts show higher reactivity than Fe-ZSM-5, fly ashsupported sulfated zirconia and ionic liquids. Furthermore, the present catalysts show superior reactivity in the alkylation of other aromatics (toluene, anisole, and xylene) compared with the catalysts reported in Table 5.7. 
Table 5.7 Catalytic reactivity of fly ash-based catalysts with other reported $\mathrm{Fe}$ containing solid catalysts in the alkylation of benzene with $\mathrm{BzCl}$ under similar reaction conditions.

\begin{tabular}{|c|c|c|c|c|c|}
\hline Catalyst & $\begin{array}{l}\text { Time } \\
(\min )\end{array}$ & $\begin{array}{c}\text { Conversion } \\
(\%)\end{array}$ & $\begin{array}{c}\text { Selectivity } \\
(\%)\end{array}$ & $\begin{array}{c}\text { Specific } \\
\text { reaction rate }\end{array}$ & $\underset{\left(x 10^{3} \min ^{-1}\right)}{k}$ \\
\hline Geo-Hyrock & 90 & 73 & 83 & 0.70 & 14.1 \\
\hline Geo-Huntly & 90 & 98 & 83 & 0.98 & 37.3 \\
\hline Fe-ZSM-5 ${ }^{[181]}$ & 100 & 98 & 50 & --- & 35.1 \\
\hline $\mathrm{Fe}-\mathrm{AlPO}_{4}{ }^{[150 \mathrm{c}]}$ & 86 & 90 & 99 & --- & 69.6 \\
\hline SZF-12 $*[157 a]$ & 240 & 87 & 100 & --- & --- \\
\hline $\mathrm{Yb}(\mathrm{OTf})_{3}{ }^{\#}$ & 1200 & 100 & 62 & --- & --- \\
\hline $\mathrm{TfOH}^{\#}$ & 1200 & 100 & 83 & --- & --- \\
\hline
\end{tabular}

${ }^{\mathrm{a}}\left(\mathrm{mmol}_{\mathrm{BzCl}} \cdot \mathrm{g}_{\mathrm{cat}}{ }^{-1} \cdot \mathrm{min}^{-1}\right)$.

* fly ash supported sulfated zirconia.

\# in [BMIM][OTf] ionic liquids solvents (reaction was carried out under different reaction conditions) ${ }^{[151]}$.

The effect of the reaction temperature on the reaction was studied in the alkylation of toluene, anisole, p-xylene and mesitylene with $\mathrm{BzCl}$ over Geo-Hyrock catalyst (Figure 5.17). A similar trend to higher reactivity with increasing temperature was observed for all the aromatics tested (toluene, p-xylene, anisole and mesitylene). 

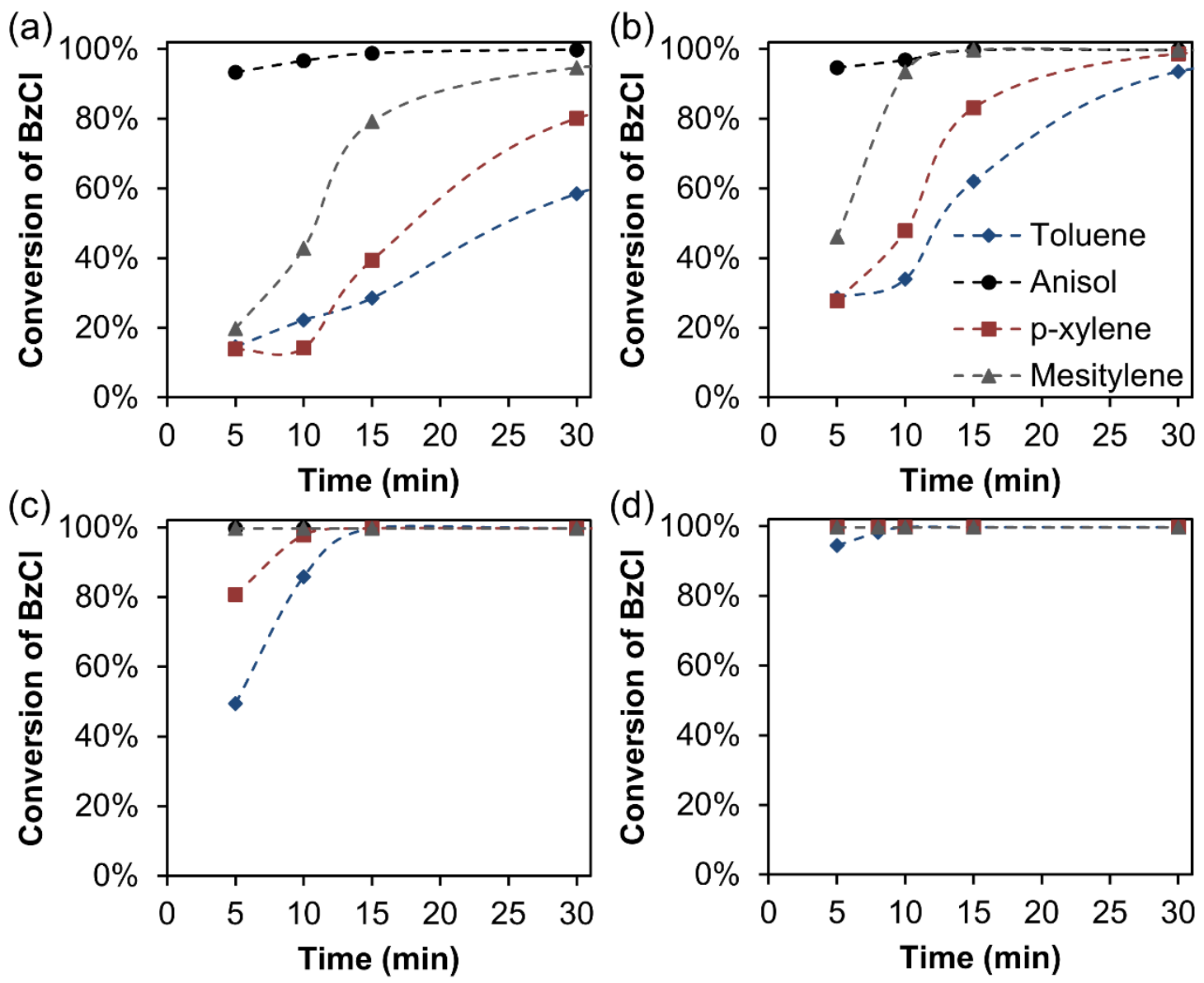

Figure 5.17 Alkylation of various aromatics with $\mathrm{BzCl}$ carried over Geo-Hyrock catalyst at different temperatures. (a) $80{ }^{\circ} \mathrm{C}$, (b) $90{ }^{\circ} \mathrm{C}$, (c) $100{ }^{\circ} \mathrm{C}$, (d) $110^{\circ} \mathrm{C}$. Aromatic: $\mathrm{BzCl}$ wt. $\%=13 ; 0.1 \mathrm{~g}$ cat.

From the data shown in Figure 5.17, the Arrhenius plot was obtained for the alkylation of toluene and xylene, from which the activation energies were calculated (Figure 5.18). Since the alkylation of anisole and mesitylene was complete in $<5 \mathrm{~min}$. and $<2 \mathrm{~min}$. at 100 and $110{ }^{\circ} \mathrm{C}$ respectively, the Arrhenius plot for the alkylation of these aromatics could not be obtained. The measured activation energy for the alkylation of toluene is lower than that determined for the clay-based catalysts $\left(152.2 \mathrm{~kJ} \mathrm{~mol}^{-1}\right)$ reflecting the higher reactivity of the fly ash-based catalysts. 


\section{$1 / \mathrm{T}(\mathrm{K})$}

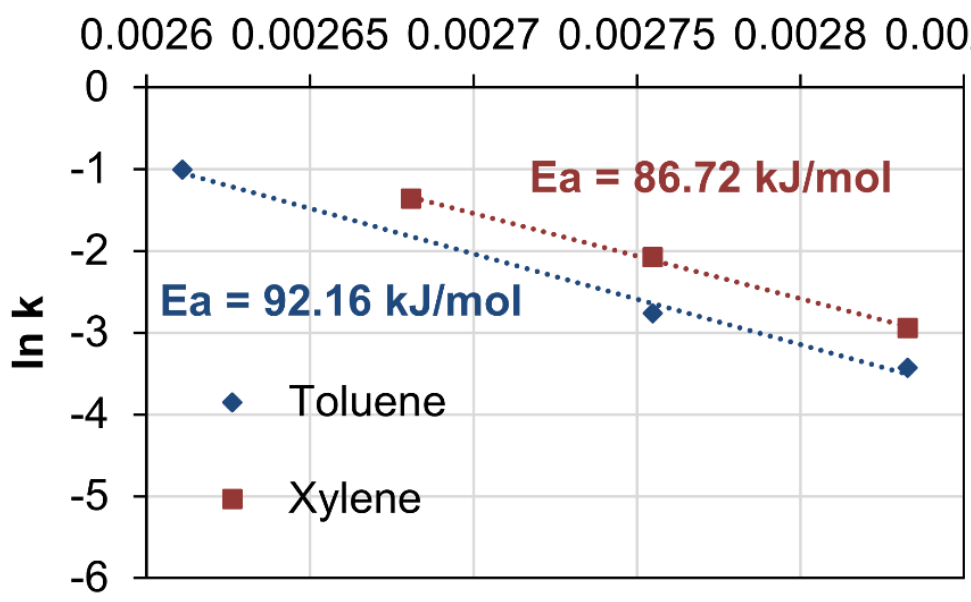

Figure 5.18 Arrhenius plot for the alkylation of toluene and p-xylene with $\mathrm{BzCl}$ over Geo-Hyrock catalyst.

The effect of the catalyst: substrate wt.\% was studied for the alkylation of toluene with $\mathrm{BzCl}$ over the Geo-Hyrock catalyst at $80{ }^{\circ} \mathrm{C}$ and $90{ }^{\circ} \mathrm{C}$ (Figure 5.8). A greater degree of conversion was obtained when more of the catalyst was used, which can be understood in terms of the availability of more active sites; this has also been observed for the clay-based catalysts (Figure 4.12).
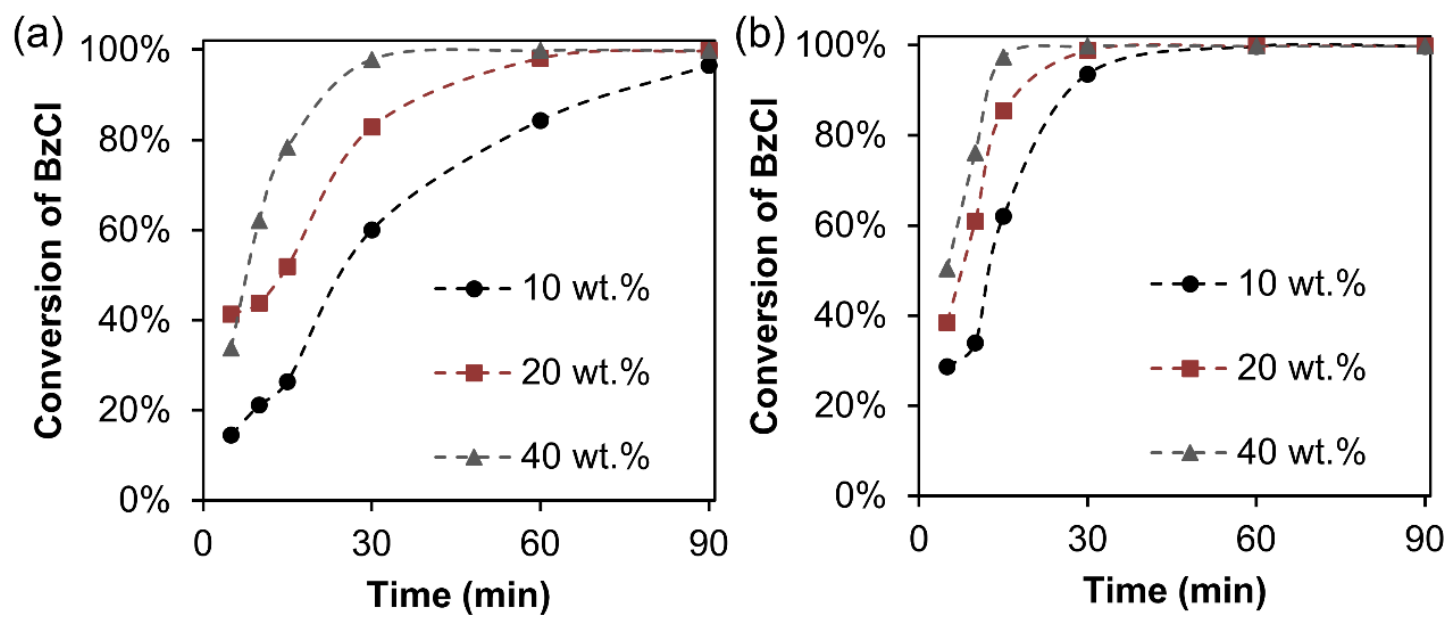

Figure 5.19 The influence of the catalyst: substrate wt.\% on the outcomes of the alkylation reaction of toluene with $\mathrm{BzCl}$ over Geo-Hyrock catalyst. (a) at $80{ }^{\circ} \mathrm{C}$, (b) at $90{ }^{\circ} \mathrm{C}$. aromatic: $\mathrm{BzCl}$ wt. $\%=13$. 
Using the data of Figure 5.19, Koros-Nowak and Madon-Boudart tests were made to ensure the absence of any mass or heat transfer limitations (Table 5.8). The TOF values obtained by the use of the different amounts of the catalyst (wt.\%) at $80{ }^{\circ} \mathrm{C}$ are similar, within a small variation range, thus satisfying Koros-Nowak model and eliminating the possible impact of any mass transfer limitations. The variation in TOF values is within an acceptable range as reported in the literature (see for example the supporting information in ref. ${ }^{[15 b]}$ ). Furthermore, the similar TOF values obtained by the use of different amounts of the catalyst (wt.\%) at a second reaction temperature $\left(90{ }^{\circ} \mathrm{C}\right)$ satisfies the Madon-Boudart model, eliminating the influence of any mass and heat transfer limitations and any other artifacts such as catalyst poisoning (see section 2.3.3.4). These results support the probability that the reaction is taking place on the surface of the fly ash-based catalysts, as discussed above.

Table 5.8 Madon-Boudart test for the mass and heat transfer limitations.

\begin{tabular}{ccc}
\hline & \multicolumn{2}{c}{ TOF $\left(\mathbf{s}^{\mathbf{- 1}}\right)^{\mathrm{a}}$} \\
\cline { 2 - 3 } Amount of $\mathrm{Fe}_{2} \mathrm{O}_{\mathbf{3}}(\mathbf{m m o l})$ & $\mathbf{8 0}{ }^{\mathbf{0}} \mathrm{C}$ & $\mathbf{9 0}{ }^{\mathbf{o}} \mathbf{C}$ \\
\hline 0.0284 & 0.106 & 0.169 \\
0.0568 & 0.110 & 0.153 \\
0.1136 & 0.078 & 0.096 \\
\hline
\end{tabular}

${ }^{\text {a }}$ measured at $10 \mathrm{~min}$ reaction time.

The reusability of the present catalysts was studied using the Geo-Hyrock catalyst in the alkylation of toluene with $\mathrm{BzCl}$ at $90{ }^{\circ} \mathrm{C}$ (Figure 5.20). The catalyst was tested up to five reaction cycles, with no sign of deactivation, and the selectivity towards the monobenzylated product (benzyltoluene) was maintained at $>95 \%$ Moreover, Figure 5.20 shows that the catalytic reactivity of the recycled catalyst is greater than in the first cycle, suggesting that a beneficial change to the catalyst structure may have taken place during use. 

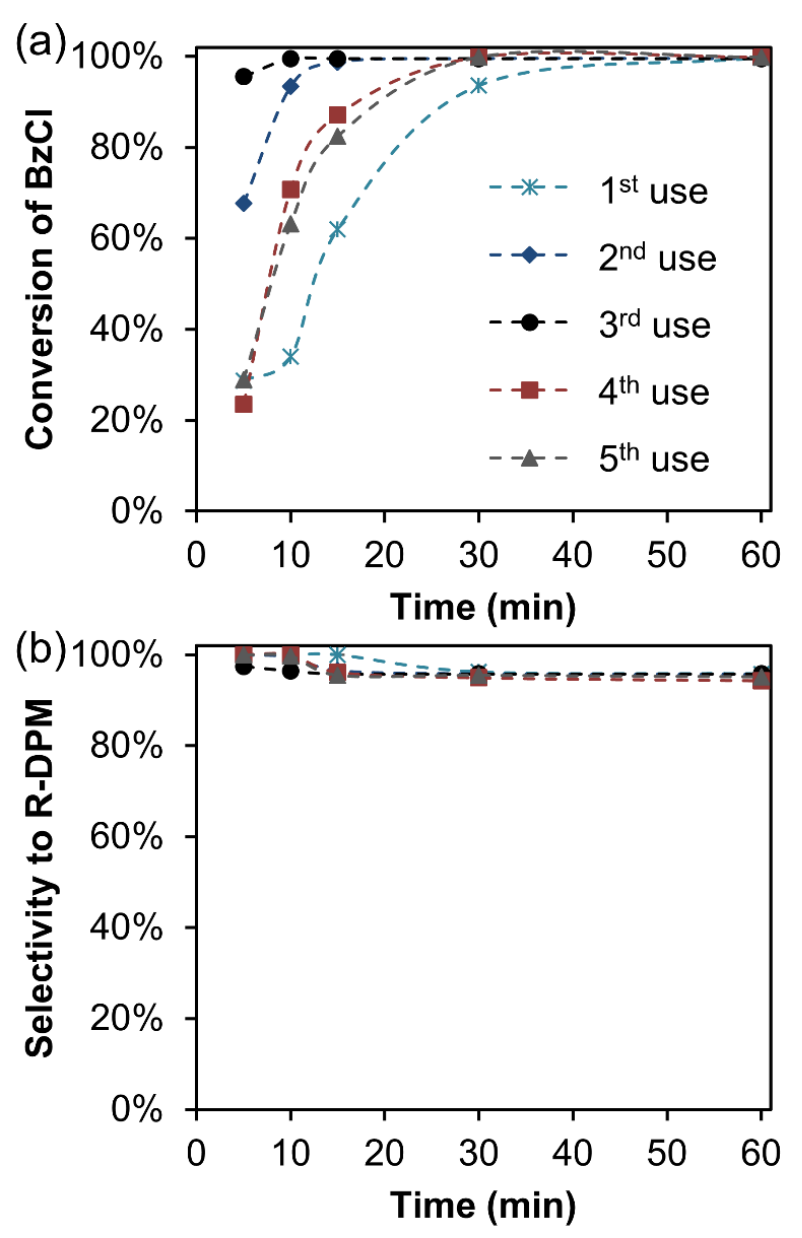

Figure 5.20 The reusability of Geo-Hyrock in alkylation of toluene with $\mathrm{BzCl}$. Reaction conditions; toluene: $\mathrm{BzCl}$ wt. $\%=13 ; \mathrm{BzCl}$ : catalysts wt. $\%=10 ; \mathrm{t}=90^{\circ} \mathrm{C}$.

The FTIR spectra of the fresh Geo-Hyrock catalyst and the reused catalyst after five reaction cycles are shown in Figure 5.21. The spectra give no indication of any adsorbed molecules on the surface of the reused catalyst, indicating that any adsorbed molecules have been degraded/desorbed from the catalyst surface after thermal reactivation (550 ${ }^{\circ} \mathrm{C}$ for $1 \mathrm{hr}$. between each reaction cycle). 


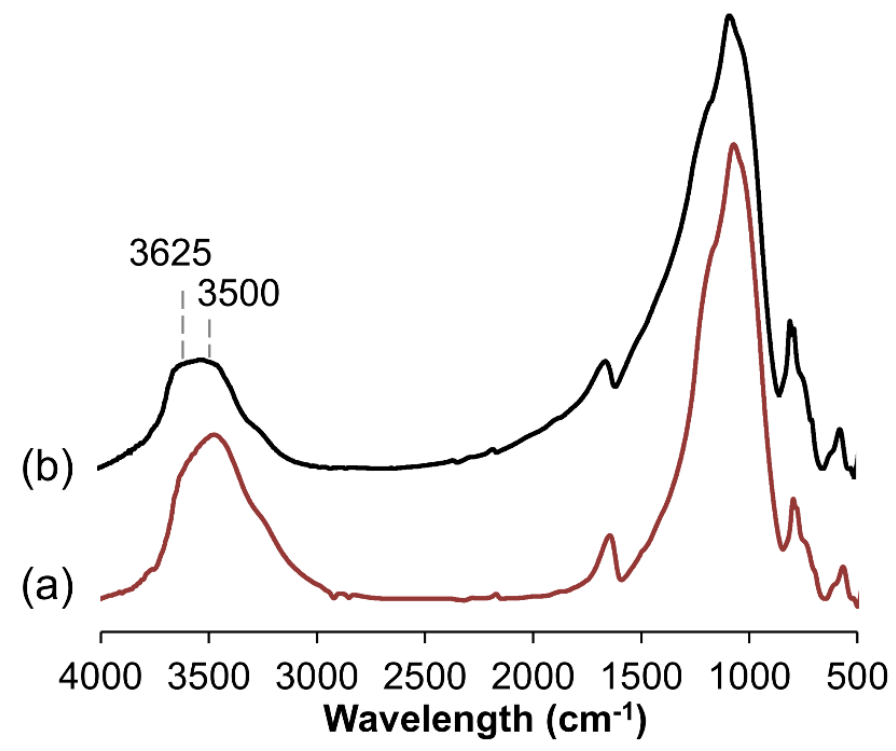

Figure 5.21 FTIR spectra of a fresh Geo-Hyrock catalyst prior use (a), and the reused catalyst after five reaction cycles (b).

However, the TGA trace of the reused catalyst (Figure 5.22) shows a decrease in the mass starting $>600{ }^{\circ} \mathrm{C}$ (above the reactivation temperature) and continuing to $>800{ }^{\circ} \mathrm{C}$ (indicated in the dashed square, Figure 5.22). This indicates that strong chemisorption is taking place, most probably within the pores of the catalyst, and suggests that poisoning of some of the less-favourable sites has occurred after the first cycle of use, explaining the higher reactivity obtained in the recycling experiments.

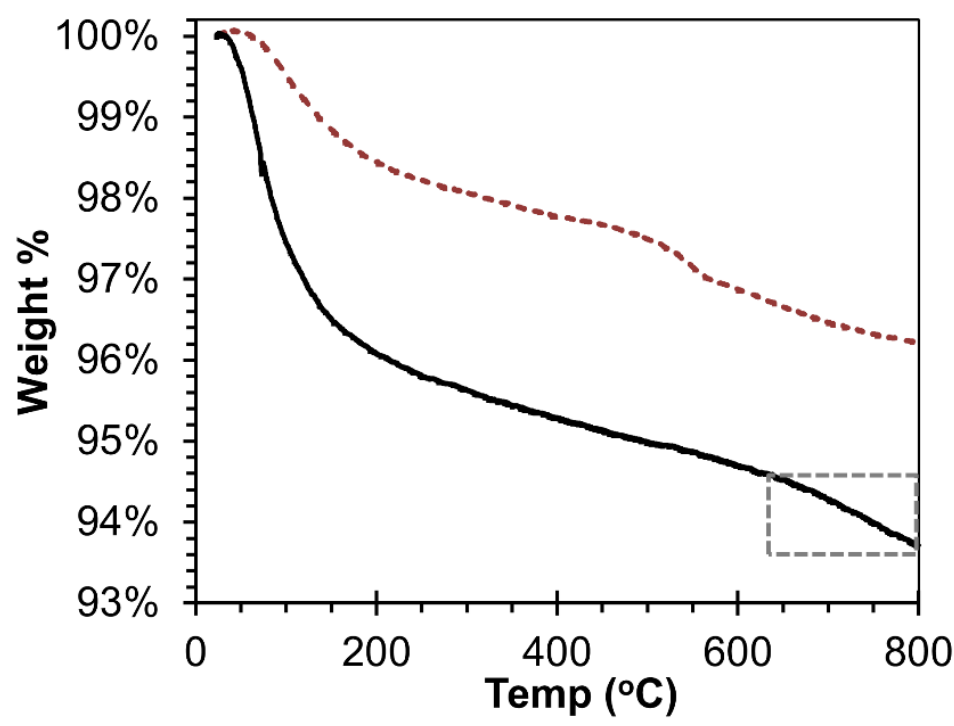

Figure 5.22 TGA of a fresh Geo-Hyrock catalyst prior use (dashed red line), and the reused catalyst after five reaction cycles (solid black line). 


\subsection{Conclusion}

This chapter reports a facile synthesis of environmentally-benign geopolymer-based heterogeneous solid acid catalysts from industrial waste materials (fly ashes). The excellent catalytic activity of the resulting catalysts shows them to represent a new class of solid catalysts with ecological and economic benefits. Four different fly ashes collected from different sources were used in the synthesis of geopolymer catalysts. Three of the four resulting fly ash-based catalysts showed excellent catalytic reactivities in the model Friedel-Crafts reaction studied in this chapter, but one catalyst showed poor reactivity which was ascribed to the low amount of $\mathrm{Fe}_{2} \mathrm{O}_{3}$ present in that particular parent fly ash. The traces of other metals present in the raw fly ashes did not seem to interfere with the catalytic performance of the fly ash-based catalysts.

The present catalysts were used in the alkylation of several arenes (benzene, toluene, anisole, p-xylene and mesitylene) and showed very high catalytic reactivities that were superior to other commonly used catalysts such as zeolites and M-zeolite, mesoporous molecular sieves and ionic liquids. The catalytic reactivities of the present materials are ascribed to the additional benefits arising from the presence of $\mathrm{Fe}_{2} \mathrm{O}_{3}$, since the geopolymer catalysts prepared from fly ashes with higher $\mathrm{Fe}_{2} \mathrm{O}_{3}$ contents were more reactive towards the model Friedel-Crafts reactions. However, the results suggest that in the alkylation of benzene in particular, the active sites are probably a combination of Lewis and Bronsted acid sites generated within the geopolymer framework, together with $\mathrm{Fe}_{2} \mathrm{O}_{3}$, since only the fly ash-based catalysts that contain a combination of these active sites were active for benzene alkylation. It was also shown that the $\mathrm{Fe}_{2} \mathrm{O}_{3}$ is enclosed within the geopolymer matrix, thereby providing an additional advantage in terms of a high level of reusability and long catalyst lifetime.

These results emphasise the suitability of fly ash as a precursor in the synthesis of highly reactive catalysts with predictable properties. Not only does the present chapter demonstrate the feasibility of synthesising active geopolymer-based catalysts from several fly ash precursors, but it also suggests a very useful means of utilizing fly ash which is a most complex but abundantly available anthropogenic material 


\section{Chapter 6}

\section{Reactivity of fly ash-based catalysts in Friedel-Crafts acylation reactions}

\subsection{Introduction}

The previous chapter showed that geopolymer-based catalysts made from fly ash precursors were highly active catalysts for the Friedel-Crafts alkylation of arenes. The high catalytic performance of substituted benzenes over a number of catalysts based on different fly ashes was ascribed to the $\mathrm{Fe}_{2} \mathrm{O}_{3}$ present in the original fly ash. It was also shown that some of these geopolymer catalysts possessed both Lewis and Bronsted acidic sites that were generated within the aluminosilicate framework after ionexchange, followed by thermal treatment. These sites, in conjunction with the $\mathrm{Fe}_{2} \mathrm{O}_{3}$ made these catalysts highly reactive in the alkylation of benzene, which usually requires stronger active sites than other substituted aromatics.

In this chapter, the catalytic reactivity of fly ash-based catalysts was investigated for another class of industrially-important and highly demanding reactions, the Friedelcrafts acylation of arenes (scheme 1). Friedel-Crafts acylation is a key step in the synthesis of aromatic ketones which are important intermediates in the pharmaceutical and agrochemical industries ${ }^{[44 \mathrm{a}]}$. 
Scheme 6.1 Friedel-Crafts acylation of arenes with benzoyl halide as the acylating agent.

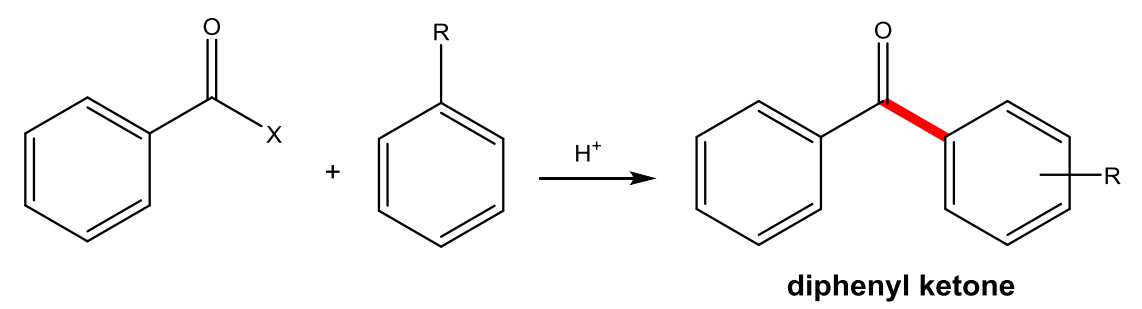

As is the case with alkylation reactions, acylation reactions are generally traditionally catalysed by conventional Lewis acids, e.g. $\mathrm{AlCl}_{3}, \mathrm{BF}_{3}, \mathrm{FeCl}_{3}$ or protic acids such as $\mathrm{H}_{2} \mathrm{SO}_{4}{ }^{[182]}$. Due to the strong coordination between the acid and the ketone product, more than the stoichiometric amount of these acids is required and the resulting product-acid complex is decomposed by quenching techniques which produce large amounts of industrial waste. This compounds the disadvantages associated with the use of such acids in terms of corrosivity, handling difficulty and reusability (section 1.2).

As is the case with alkylation reactions described in Chapters 4 and 5, Lewis or Bronsted acidic sites, or a combination of both, are required to catalyse the acylation reactions over solid acids. Other metal oxides (e.g. $\left.\mathrm{Fe}_{2} \mathrm{O}_{3}\right){ }^{[183]}$ have also been reported as active catalysts for acylation reactions. Both the mechanisms that have been used to describe the alkylation reactions are usually also applied to the acylation reaction pathways; these occur via either the typical electrophilic substitution mechanism, in the presence of Lewis or Bronsted acid sites (Figure 4.2), or via the redox (radical) mechanism when metal oxides are used (Figure 5.16).

Several solid acid catalysts have been developed and applied to the acylation of aromatics; these include zeolites, clays, mixed oxides and heteropolyacids (see for example ref. ${ }^{[44 a]}$ and the references therein). Recently, a variety of functionalised solid catalysts such as $\mathrm{Fe}_{2} \mathrm{O}_{3}$ supported on $\mathrm{H}-\mathrm{Y}$ zeolite ${ }^{[183]}$, heteropolyacids encapsulated into a zeolite framework ${ }^{[184]}$, metal organic frameworks (MOFs) ${ }^{[185]}$, functionalised organic polymers (e.g. poly(4-vinylpyridine)), supported trifluoromethanesulfonic acid ${ }^{[186]}$, magnetic nanopowders (e.g. $\left.\mathrm{CuFe}_{2} \mathrm{O}_{4}\right)^{[187]}$, and ionic liquids ${ }^{[188]}$ have been used, showing high reactivities in the acylation of different aromatics. 
In this Chapter, geopolymer-based solid acid catalysts prepared from fly ash precursors, containing a combination of Bronsted and Lewis acidic sites and $\mathrm{Fe}_{2} \mathrm{O}_{3}$ are shown to perform as active catalysts for the Friedel-Crafts acylation of substituted benzenes using benzoylchloride (BzoylCl) as the acylating agent.

The work described in this chapter was initiated only very recently to demonstrate that the use of geopolymer-based catalysts can be extended to a wider variety of catalytic processes. Thus, further work will be required to optimise the results and provide a more precise evaluation of the catalytic reactivity to allow a sensible comparison with other previously reported catalysts for this particular reaction.

\subsection{Experimental}

\subsubsection{Catalysts preparation}

Two of the four fly ash-based geopolymer catalysts used previously (Geo-Huntly and Geo-Hyrock) were tested in the acylation reactions of several arenes. The synthesis of these fly ash-based geopolymers and the subsequent preparation of the catalysts is described in detail in Chapter 2 (section 2.1.1 and 2.1.2).

\subsubsection{Catalytic reactions}

The reaction was carried out as described in Chapter 2 (section 2.3.1). In a typical run, $1 \mathrm{ml}$ of BzoylCl was mixed with $13 \mathrm{ml}$ of the aromatic compound (toluene, anisole, $\mathrm{p}$ xylene or mesitylene) and the mixture was set to the desired temperature followed by the addition of $0.1 \mathrm{~g}$ of the activated catalyst. This point is taken as the starting time of the reaction. The reaction analysis and the catalytic calculations are described in Chapter 2 (section 2.33). 


\subsection{Results and discussion}

The catalytic reactivity of the fly ash-based catalysts in the Friedel-Crafts acylation reactions of several substituted benzenes (toluene, anisole, p-xylene, and mesitylene with BzoylCl as the acylating agent) was initially investigated using the Geo-Huntly catalyst (Figure 6.1) because of its higher acidity and catalytic reactivity in the alkylation reactions compared to the other fly ash-based catalysts.

As seen in Figure 6.1, Geo-Huntly shows poor reactivity in the acylation of toluene and p-xylene, with $\sim 14$ and 30\% conversion of BzoylCl, respectively, after 3hr. reaction time (Table 6.1). However, in the acylation of mesitylene and anisole the catalyst displayed high reactivity, giving almost complete conversion of BzoylCl within $2 \mathrm{hr}$. in the acylation of anisole, and $\sim 95 \%$ conversion in the acylation of mesitylene. In addition, excellent selectivities were obtained in both the acylation of anisole and mesitylene (typically $>99 \%$ towards the mono-acylated products). In the case of anisole, the main product formed was the (4-methoxyphenyl)-phenylmethanone (compound $2 \mathrm{~b}$, scheme 6.2 ) with selectivity up to $97 \%$ and only $3 \%$ selectivity to the ortho isomer (scheme 6.2,1a). However, lower selectivities were obtained in the acylation of toluene and p-xylene with the principal by-product being 2-oxo-2phenylethylformate.

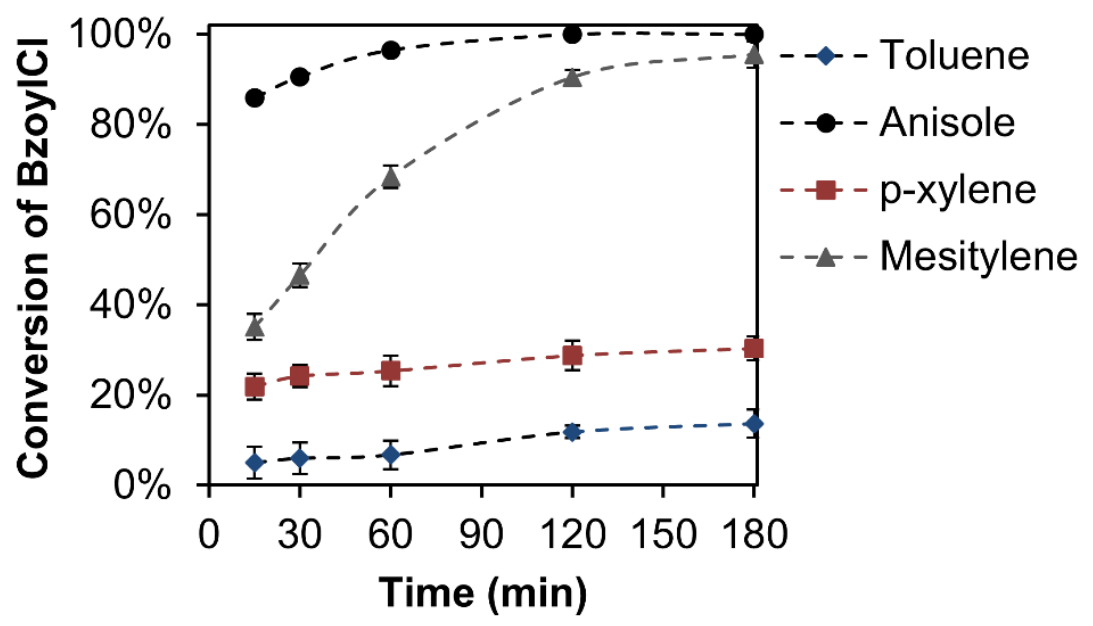

Figure 6.1 Catalytic reactivity of Geo-Huntly in the acylation of several aromatics with BzoylCl. Reaction conditions; $13 \mathrm{ml}$ aromatic, $1 \mathrm{ml}$ BzoylCl, $0.1 \mathrm{~g}$ catalyst; $\mathrm{T}=130{ }^{\circ} \mathrm{C}$ for all aromatics except toluene $110^{\circ} \mathrm{C}$. 
Scheme 6.2 The outcomes of the acylation reaction of different aromatics with BzoylCl.<smiles>COc1ccccc1C(=O)c1ccccc1</smiles>

1a<smiles>COc1ccc(C(=O)c2ccccc2)cc1</smiles><smiles>Cc1ccccc1C(=O)c1ccccc1</smiles>

2a<smiles>Cc1ccc(C(=O)c2ccccc2)cc1</smiles>

2b<smiles>Cc1cc(C)c(C(=O)c2ccccc2)c(C)c1</smiles><smiles>Cc1ccc(C)c(C(=O)c2ccccc2)c1</smiles>

Table 6.1 Catalytic reactivity of Geo-Huntly in the acylation of several aromatics with BzoylCl as the acylating agent ${ }^{\mathrm{a}}$.

\begin{tabular}{|c|c|c|c|c|c|}
\hline Substituent & $\begin{array}{c}\text { Conversion } \\
(\%)\end{array}$ & $\begin{array}{c}\text { Selectivity } \\
(\%)\end{array}$ & $\begin{array}{c}\text { Specific } \\
\text { reaction rate }\end{array}$ & $\frac{k}{\left(x_{10}^{3} \min ^{-1}\right)}$ & TON \\
\hline Toluene $^{\mathrm{c}}$ & 14 & $20(2 b)^{*}$ & 0.07 & 0.6 & 23 \\
\hline Anisole & $100^{d}$ & 97 (1b) & 0.47 & 18.1 & 164 \\
\hline p-xylene & 30 & 91 (d) & 0.15 & 0.7 & 51 \\
\hline Mesitylene & 96 & $100(\mathrm{c})$ & 0.46 & 14.1 & 159 \\
\hline Anisole $^{\mathrm{e}}$ & 37 & $92(1 b)$ & 0.18 & 0.5 & 19 \\
\hline
\end{tabular}

${ }^{a}$ Reaction conditions; $13 \mathrm{ml}$ aromatic, $1 \mathrm{ml}$ BzoylCl, $0.1 \mathrm{~g}$ catalyst; $\mathrm{T}=130{ }^{\circ} \mathrm{C} ; \mathrm{t}=3 \mathrm{hr}$.

$\mathrm{b}\left(\mathrm{mmol}_{\mathrm{BzCl}} \cdot \mathrm{g}_{\mathrm{cat}}{ }^{-1} \cdot \mathrm{min}^{-1}\right)$.

${ }^{\mathrm{c}} \mathrm{T}=110^{\circ} \mathrm{C}$.

${ }^{d} \mathrm{t}=2 \mathrm{hr}$.

e over H-Y zeolite.

* the figures in parentheses refer to compounds in scheme 6.2.

When $\mathrm{H}-\mathrm{Y}$ zeolite was tested under identical reaction conditions, it showed poor reactivity, with $\sim 37 \%$ conversion of BzoylCl and $\sim 90 \%$ selectivity to product $1 \mathrm{~b}$ (Table 6.1). The principal by product was again identified as 2-oxo-2-phenylethylformate. 
The results of Figure 6.1 show a similar trend of reactivity in the acylation reaction to that observed in the alkylation reactions (section 5.3.2) where high catalytic reactivity was achieved for the alkylation of aromatics with high electron density on the benzene ring.

The Langmuir-Hinshelwood pseudo-first-order kinetic model was applied to the acylation of all the aromatics investigated here (Figure 6.2), from which the reaction constants were measured as summarised in Table 6.1.

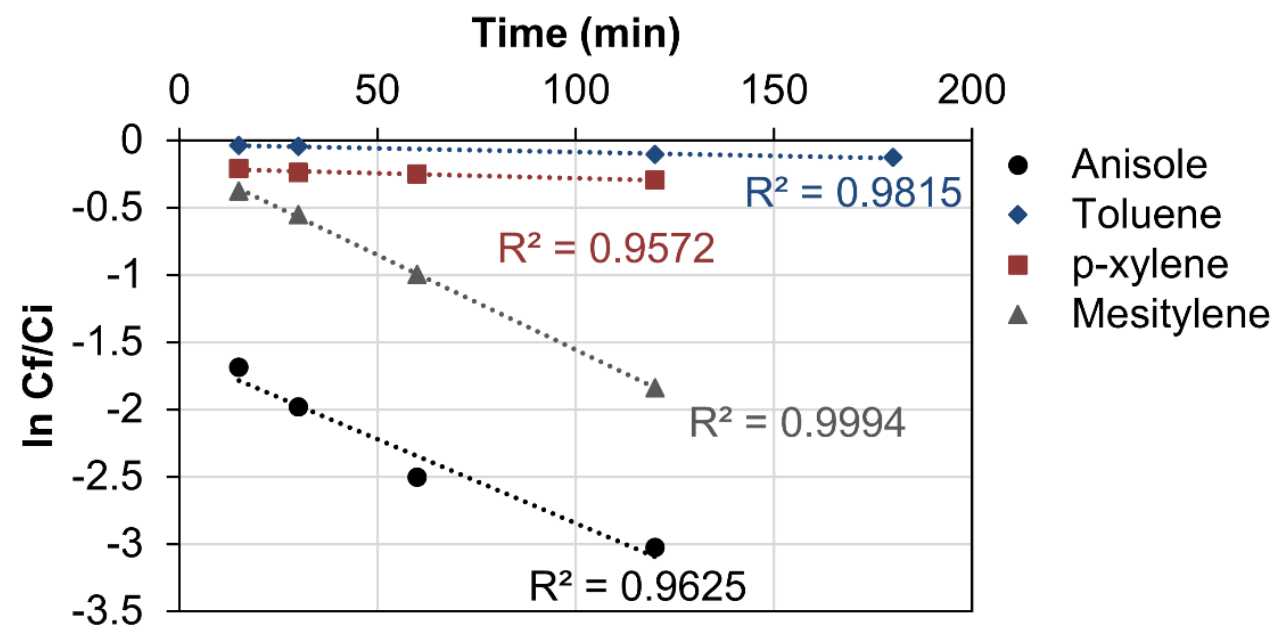

Figure 6.2 Langmuir-Hinshelwood pseudo-first-order kinetic model for the acylation of several aromatics over the Geo-Huntly catalyst. Reaction conditions as described for Figure 6.1.

The influence of the reaction temperature was investigated in the acylation of anisole with BzoylCl over Geo-Huntly and Geo-Hyrock catalysts (Figures 6.3a and 6.3b respectively). Similar catalytic reactivities were obtained with both catalysts at 110 and $120{ }^{\circ} \mathrm{C}$, but at $130{ }^{\circ} \mathrm{C}$, and improvement in the conversion of BzoylCl was observed with both catalysts. Figure 6.3 shows that higher reactivity was achieved with the GeoHuntly catalyst compared with the Geo-Hyrock catalyst; this is ascribed to the higher acidity and $\mathrm{Fe}_{2} \mathrm{O}_{3}$ content of the former, as discussed in the previous Chapter (sections 5.3.1 and 5.3.2). 

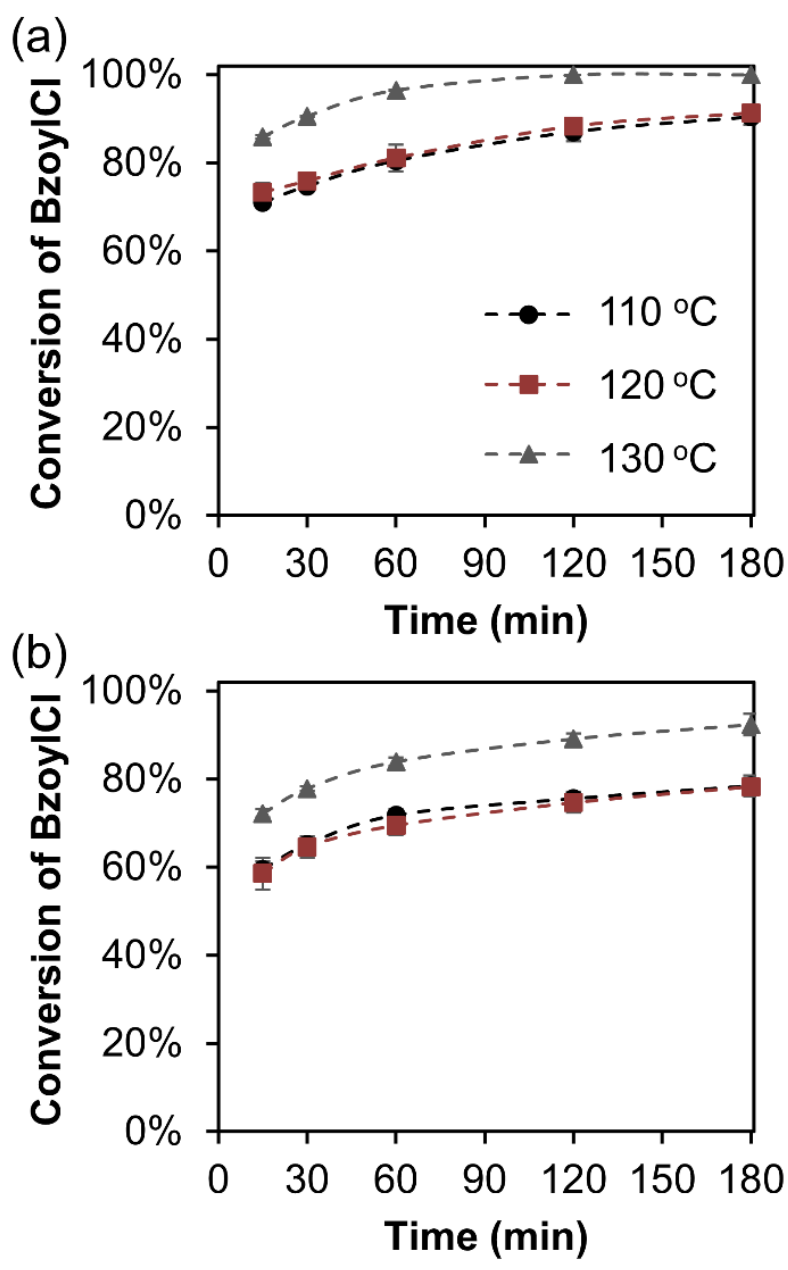

Figure 6.3 Catalytic reactivity of Geo-Huntly catalyst (a) and Geo-Hyrock catalyst (b) in the Friedel-Crafts acylation of anisole with BzoylCl at different reaction temperatures. Reaction conditions; $13 \mathrm{ml}$ anisole, $1 \mathrm{ml}$ BzoylCl, $0.1 \mathrm{~g}$ catalyst.

Higher activity was observed when more catalyst is used (Figure 6.4). The effect of using more catalyst is most apparent at $120{ }^{\circ} \mathrm{C}$ (Figure 6.4a) in which applying higher cat. wt.\% results in higher conversion $\%$ of the substrate. However, when the reaction is carried out at $130{ }^{\circ} \mathrm{C}$ (Figure 6.4b), only a slight improvement in the catalytic reactivity was observed when 8 and 16 catalyst: substrate wt.\% were used, the main difference is noticed within the first 60 min reaction time. 

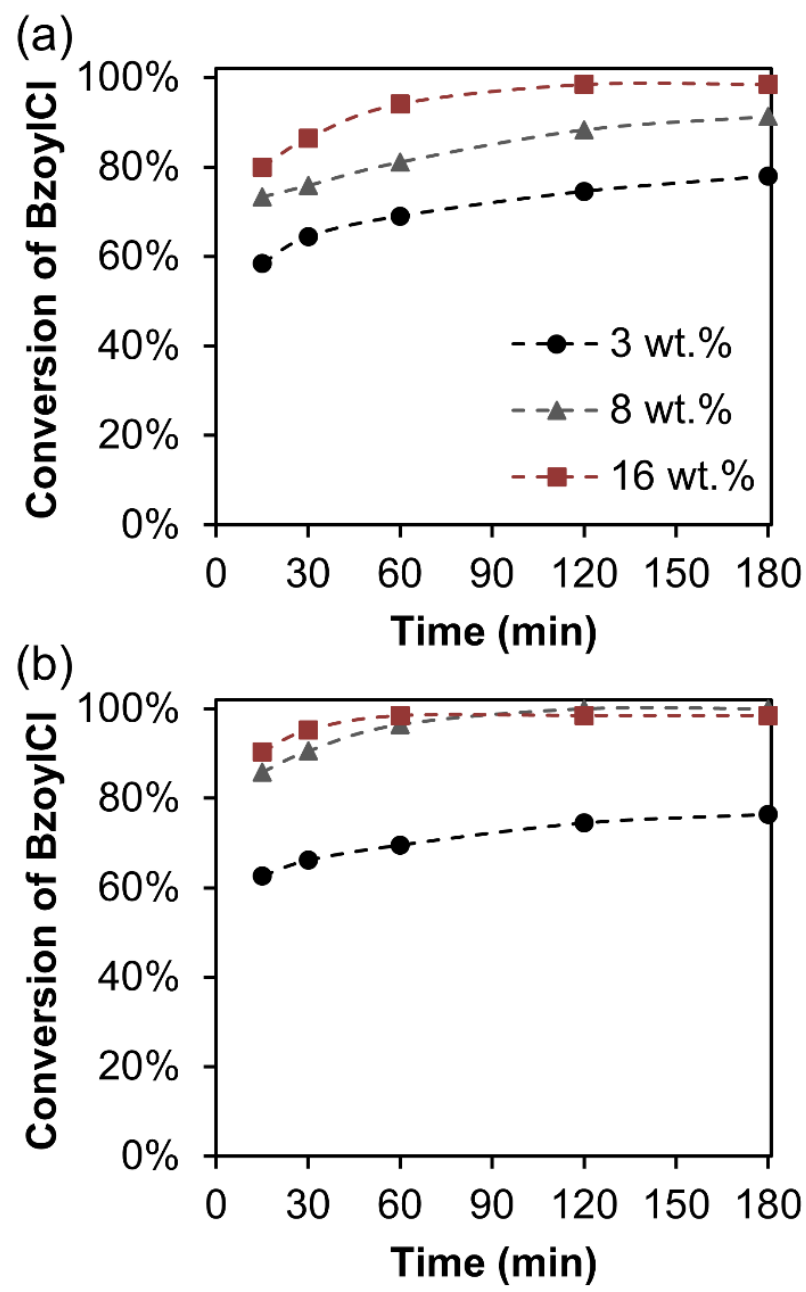

Figure 6.4 Influence of the catalyst: substrate wt.\% on the acylation reaction of anisole with BzoylCl over Geo-Huntly catalyst. (a) at $120{ }^{\circ} \mathrm{C}$, (b) at $130{ }^{\circ} \mathrm{C}$. Reaction conditions; anisole: BzoylCl wt.\% 13

The catalytic performance of the Geo-Huntly catalyst in the acylation of anisole and mesitylene was comparable with or superior to other recently reported solid catalysts such as $\mathrm{CuFe}_{2} \mathrm{O}_{4}$ magnetic nanopowders ${ }^{[187]}, \mathrm{H}_{3} \mathrm{PW}_{12} \mathrm{O}_{40}$ - encapsulated into ZIF-67 zeolite framework $^{[184]}$, and poly(4-vinylpyridine) supported trifluoromethanesulfonic) ${ }^{[186]}$. Fly ash geopolymer-based catalysts provide additional advantages over these solid catalysts since they can readily be synthesised in an energy-efficient process from industrial waste precursors, whereas other solid catalysts are costly and require sophisticated synthesis techniques. 


\subsection{Conclusion}

This chapter has demonstrated the catalytic reactivity of fly ash-based geopolymer catalysts in the highly demanding Friedel-Crafts acylation of arenes. Two geopolymerbased catalysts prepared from two different fly ashes (F-class Hyrock and C-class Huntly fly ashes) were tested, and both catalysts showed high reactivity with excellent selectivity in the acylation of anisole and mesitylene; these results were superior to the catalytic performance of H-Y zeolite. However, poor reactivities were observed in the acylation of toluene and p-xylene, possibly due to the need for stronger sites to enable these reactions to proceed.

The fly ash-based geopolymer catalysts exhibited superior or at least comparable results with other recently reported catalysts which are costly and complicated to synthesise; thus the new fly ash-based catalysts have an excellent potential as environmentallyfriendly heterogeneous solid catalysts. 


\section{Chapter 7}

\section{Conclusion and future work}

\subsection{Conclusion}

This thesis reports the facile synthesis and characterisation of porous aluminosilicate inorganic polymers (geopolymers) as a novel class of cost-efficient and environmentally friendly heterogeneous solid acid catalysts. Geopolymers were prepared from several different precursors and the necessary acid sites (Bronsted and Lewis) were incorporated into their framework by ammonium ion-exchange followed by thermal treatment which decomposes the $\mathrm{NH}_{3}$ producing the "H-form" of geopolymers. In some cases, the synthesised geopolymers were subjected to post synthetic demetallation (dealumination and desilication) which resulted in improved porosity and acidity and thus catalytic reactivity.

The geopolymers were initially synthesised from natural clay mineral (halloysite) and the influence was investigated of different compositions (mainly the $\mathrm{Si} / \mathrm{Al}$ ratio) and the alkali activators ( $\mathrm{Na}$ or $\mathrm{K}$ ) on the resulting geopolymer characteristics. The resulting geopolymers exhibited a number of different porosities; micro, meso and a combination of both (hierarchical geopolymers). Thermal treatments generated different acidic silanol groups, including bridging hydroxyls (Bronsted sites) as well as extraframework aluminium (EFAl) that usually acts as Lewis acid sites, allowing geopolymers to be applied to different catalytic reactions where different active sites are required.

The catalytic performance of the clay-based geopolymer catalysts was first investigated in the Beckmann rearrangement of cyclohexanone oxime to $\varepsilon$-caprolactam (Chapter 3). 
This reaction most probably requires weakly acidic silanol groups (hydrogen-bonded silanol groups, 'silanol nests' and vicinal silanols); therefore the ion-exchanged geopolymers were thermally treated at $450{ }^{\circ} \mathrm{C}$ to generate these silanol groups with the minimum amount of EFAl that undergo hydrolysis to the oxime, producing undesired side products. High rates of conversion to the oxime were achieved with all the geopolymer-based catalysts; however, high selectivity towards the desired product was only obtained over a post-synthetic treated geopolymer catalyst that possessed a high surface area $\left(\sim 200 \mathrm{~m}^{2} / \mathrm{g}\right)$. the catalytic performance of these materials in Beckmann rearrangement

The reactivity of the clay-based geopolymer catalysts was also investigated in catalytic reactions that require Bronsted and Lewis acidic sites, such as the Friedel-Crafts alkylation of aromatics (Chapter 4). For this reaction, the ion-exchanged geopolymers were thermally treated at $550{ }^{\circ} \mathrm{C}$ to completely decompose the $\mathrm{NH}_{3}$ and achieve severe destruction of the hydroxyl groups, with the production of a high concentration of EFAl. It was shown that the post-synthetic treatment was essential to produce the required higher porosity and acidity. High reactivity was achieved; the alkylation of substituted benzenes (toluene, anisole, p-xylene and mesitylene) with benzylchloride as the alkylating agent was almost complete within two hours, with $\sim 100 \%$ selectivity towards monobenzylated products over all the modified geopolymers.

Furthermore, highly reactive geopolymer-based catalysts were synthesised from industrial waste precursors (fly ashes). These gave excellent performance in the FriedelCrafts alkylation of benzene and substituted benzenes with benzylchloride as the alkylating agent (Chapter 5). The reactivity of these catalysts was ascribed to a combination of active sites, namely the $\mathrm{Fe}_{2} \mathrm{O}_{3}$ present in the raw fly ash, together with the Bronsted and Lewis acidic sites generated within the geopolymer framework. The influence of the physical and chemical properties of the fly ash on the catalytic activities was investigated by using different fly ashes collected from various sources. Of the four fly ash-based catalysts, only one performed poorly in the reaction conditions of this study; this was ascribed to the very low $\mathrm{Fe}_{2} \mathrm{O}_{3}$ content $(<1.0 \mathrm{wt} . \%)$ in this fly ash. Other metal oxides present in the raw fly ash do not appear to negatively affect the catalytic performance of these fly ash-based catalysts (for instance, reactive fly ash-based geopolymer catalysts were obtained from both C-class and F-class fly ashes). High 
catalytic reactivities of fly ash-based catalysts were also achieved in the Friedel-Crafts acylation reactions of anisole and mesitylene with benzoylchloride as the acylating agent (Chapter 6).

The clay-based and fly ash-based geopolymer catalysts were recycled several times without significant loss of reactivity, emphasising the high chemical and thermal stability of these catalysts. These results also show that geopolymer catalysts do not suffer the drawbacks of supported catalysts, which arise from the weak interaction between the active species and the host, leading to loss of reactivity due to leaching of the active sites.

The catalytic performance of the geopolymer-based catalysts is comparable with and sometimes superior to other commonly used solid acids, including zeolites, mesoporous silicates (Al-MCM-41), mixed oxides and other functionalised solid catalysts. The present work indicates the potential of geopolymers as new, cost efficient, readily synthesised and environmentally-friendly heterogeneous solid acid catalysts for fine chemical applications.

\subsection{Future work}

The development of hierarchically organised porous catalysts that possess interconnected pores at several length scales; micro (<2 nm), meso $(2-50 \mathrm{~nm})$ and macro $(>50 \mathrm{~nm})$ is a rapidly growing area of research. Catalysts with such multilevel architectures provide high interfacial areas and overcome diffusional limitations associated with microporous materials. However, the synthesis of these materials, following the more precise "bottom-up" approaches, often requires the use of expensive reagents that elevates the overall preparation costs which might militate against their commercialisation.

In this context, it would be interesting to work on the development of inexpensive self-supported geopolymer monoliths with well-defined pores for catalysis applications. For this task, hard templating techniques (nano-casting) could be applied using various ecologically benign templating agents such as, for example, nano-fibres derived from natural resources (e.g. cellulose or polypeptide chains extracted from plants and wool fibres respectively). 
Monolithic geopolymer heterogeneous catalysts could have substantial industrial potential considering the ability to generate acid-base or redox active centres within their frameworks. Furthermore, these materials would possess some advantages over common mesoporous materials such as MCM-41, particularly, in terms of their excellent thermal and hydrothermal stabilities, and lower preparation costs.

Another approach would be the development of new acid-base geopolymerbased heterogeneous catalysts for efficient production of sustainable fuel (biodiesel). Recent estimates of the limited fossil fuel reserves and the amount of $\mathrm{CO}_{2}$ produced by burning such carbon resources, with its contribution to the climate change, have urged the need for renewable and clean energy alternatives. In this regards, biodiesel has attracted much interests as a green and low-cost alternative fuel with potential to meet the future demands. Biodiesel is usually produced via transesterification of triglycerides from animal fat or vegetable oil over solid bases, most commonly over alkali or alkaline earth oxides (e.g. $\mathrm{Na}_{2} \mathrm{O}$ and $\mathrm{CaO}$ ) loaded on porous supports. Despite their efficiency, those catalysts suffer from the tendency of their active species to leach from the host in aqueous solutions, causing deactivation and affecting the catalyst reusability.

In this perspective, it would be interesting to investigate the catalytic reactivities of porous aluminosilicate inorganic polymers synthesised from C-class fly ash (fly ash with $>8$ wt.\% $\mathrm{CaO}$ ) as solid base catalysts for production of biodiesel. Since the active sites are present in the raw material of the catalysts, they are expected to be enclosed within the polymer matrix, thereby providing an additional advantage in terms of higher level of reusability and longer catalyst lifetime. In addition to their high anticipated reactivity, these catalysts will contribute towards effective utilisation of one of the most abundant anthropogenic materials.

Furthermore, A deeper understanding of the geopolymer catalyst structure could help to produce geopolymers with the desired properties that suit specific catalytic applications. This could be achieved by applying theoretical approaches such as density functional theory in conjunction with experimental data obtained from solid state NMR, UV-Vis spectroscopy of probe metals and FTIR. 


\section{Appendix A}

Table A1: Elements oxides wt.\% of all the prepared and the ion-exchanged fly ash-based catalysts a

\begin{tabular}{|c|c|c|c|c|c|c|c|c|c|c|c|c|c|}
\hline Sample & $\mathrm{Fe}_{2} \mathrm{O}_{3}$ & MnO & $\mathrm{TiO}_{2}$ & $\mathrm{CaO}$ & $\mathbf{K}_{2} \mathbf{O}$ & $\mathrm{SO}_{3}$ & $\mathbf{P}_{2} \mathbf{O}_{5}$ & $\mathrm{SiO}_{2}$ & $\mathrm{Al}_{2} \mathrm{O}_{3}$ & MgO & $\mathrm{Na}_{2} \mathrm{O}$ & L.O.I. & SUM \\
\hline Hyrock-Geo & 4.25 & 0.06 & 0.70 & 0.80 & 1.04 & 0.06 & 0.13 & 46.29 & 20.67 & 0.45 & 16.74 & 7.96 & 99.16 \\
\hline Hyrock-Geo-NH${ }_{4}^{+}$ & 4.53 & 0.06 & 0.82 & 0.86 & 0.22 & 0.01 & 0.07 & 54.08 & 24.70 & 0.46 & 4.16 & 9.81 & 89.97 \\
\hline Huntly Geo & 6.98 & 0.05 & 0.79 & 10.51 & 0.44 & 0.33 & 0.17 & 40.71 & 16.96 & 1.89 & 11.28 & 8.66 & 98.78 \\
\hline Huntly $\mathrm{Geo}-\mathrm{NH}_{4}{ }^{+}$ & 8.26 & 0.06 & 0.93 & 8.84 & 0.14 & $<0.01$ & 0.17 & 48.88 & 20.50 & 2.06 & 0.43 & 9.52 & 99.79 \\
\hline Geo-GS & 8.14 & 0.10 & 1.09 & 2.34 & 0.32 & 0.07 & 0.66 & 40.91 & 19.36 & 1.02 & 15.75 & 9.53 & 99.29 \\
\hline G.S. $\mathrm{Geo}-\mathrm{NH}_{4}^{+}$ & 9.05 & 0.11 & 1.27 & 2.54 & 0.10 & $<0.01$ & 0.36 & 48.90 & 23.27 & 1.12 & 1.20 & 11.90 & 99.82 \\
\hline Mt Piper Geo & 0.77 & 0.01 & 0.62 & 0.16 & 1.46 & $<0.01$ & 0.07 & 56.43 & 23.06 & 0.16 & 11.23 & 5.67 & 99.65 \\
\hline Mt Piper Geo $-\mathrm{NH}_{4}^{+}$ & 0.84 & 0.02 & 0.69 & 0.17 & 0.92 & $<0.01$ & 0.03 & 61.01 & 25.34 & 0.16 & 0.60 & 9.78 & 99.57 \\
\hline
\end{tabular}

a determined by XRF 


\section{Bibliography}

[1] I. Fechete, Y. Wang and J. C. Védrine, Catalysis Today 2012, 189, 2-27.

[2] B. Lindström and L. J. Pettersson, CATTECH 2003, 7, 130-138.

[3] O. Deutschmann, H. Knözinger, K. Kochloefl and T. Turek in Heterogeneous Catalysis and Solid Catalysts, Wiley-VCH Verlag GmbH \& Co. KGaA, 2000.

[4] R. Whyman, Applied Organometallic Chemistry 2005, 19, 994-994.

[5] J. C. Védrine, Applied Catalysis A: General 2014, 474, 40-50.

[6] Acmite Market Intelligence, Market Reports, 2015.

[7] a) J.-i. Kadokawa and S. Kobayashi, Current Opinion in Chemical Biology 2010, 14, 145-153; b) F. Lopez-Gallego and C. Schmidt-Dannert, Current Opinion in Chemical Biology 2010, 14, 174-183.

[8] M. Beller, A. Renken and R. A. van Santen, Catalysis: From Principles to Applications, Wiley, 2012, p.

[9] C. Copéret, M. Chabanas, R. Petroff Saint-Arroman and J.-M. Basset, Angewandte Chemie International Edition 2003, 42, 156-181.

[10] J. H. Clark, Accounts of Chemical Research 2002, 35, 791-797.

[11] J. H. Clark, Green Chemistry 1999, 1, 1-8.

[12] R. A. Sheldon, I. W. C. E. Arends and U. Hanefeld in Solid Acids and Bases as Catalysts, Wiley-VCH Verlag GmbH \& Co. KGaA, 2007.

[13] K. Tanabe and W. F. Hölderich, Applied Catalysis A: General 1999, 181, 399-434. 
[14] J. D. A. Pelletier and J.-M. Basset, Accounts of Chemical Research 2016, 49, 664677.

[15] J. Weitkamp, Solid State Ionics 2000, 131, 175-188.

[16] Y. Li and J. Yu, Chemical Reviews 2014, 114, 7268-7316.

[17] S. Goel, S. I. Zones and E. Iglesia, Chemistry of Materials 2015, 27, 2056-2066.

[18] X. Meng and F.-S. Xiao, Chemical Reviews 2014, 114, 1521-1543.

[19] a) A. Corma, Current Opinion in Solid State and Materials Science 1997, 2, 63-

75; b) J. L. White and M. J. Truitt, Progress in Nuclear Magnetic Resonance Spectroscopy 2007, 51, 139-154.

[20] L. A. Pine, P. J. Maher and W. A. Wachter, Journal of Catalysis 1984, 85, 466476.

[21] J. Jiao, J. Kanellopoulos, W. Wang, S. S. Ray, H. Foerster, D. Freude and M. Hunger, Physical Chemistry Chemical Physics 2005, 7, 3221-3226.

[22] J. Brus, L. Kobera, W. Schoefberger, M. Urbanová, P. Klein, P. Sazama, E. Tabor, S. Sklenak, A. V. Fishchuk and J. Dědeček, Angewandte Chemie International Edition 2015, 54, 541-545.

[23] N. Rahimi and R. Karimzadeh, Applied Catalysis A: General 2011, 398, 1-17.

[24] T. F. Degnan Jr, C. M. Smith and C. R. Venkat, Applied Catalysis A: General 2001, 221, 283-294.

[25] U. Olsbye, S. Svelle, M. Bjørgen, P. Beato, T. V. W. Janssens, F. Joensen, S. Bordiga and K. P. Lillerud, Angewandte Chemie International Edition 2012, 51, 58105831.

[26] a) J. Perez-Ramirez, C. H. Christensen, K. Egeblad, C. H. Christensen and J. C. Groen, Chemical Society Reviews 2008, 37, 2530-2542; b) Y. Wan and Zhao, Chemical Reviews 2007, 107, 2821-2860. 
[27] a) K. Leng, S. Sun, B. Wang, L. Sun, W. Xu and Y. Sun, Catalysis Communications 2012, 28, 64-68; b) Y. Wang, Y. Sun, C. Lancelot, C. Lamonier, J.C. Morin, B. Revel, L. Delevoye and A. Rives, Microporous and Mesoporous Materials 2015, 206, 42-51.

[28] M. E. Davis, Nature 2002, 417, 813-821.

[29] P. Van Der Voort, C. Vercaemst, D. Schaubroeck and F. Verpoort, Physical Chemistry Chemical Physics 2008, 10, 347-360.

[30] R. Ryoo and S. Jun, The Journal of Physical Chemistry B 1997, 101, 317-320.

[31] J. Zhu, X. Meng and F. Xiao, Frontiers of Chemical Science and Engineering 2013, 7, 233-248.

[32] M. S. Holm, E. Taarning, K. Egeblad and C. H. Christensen, Catalysis Today 2011, 168, 3-16.

[33] a) D. Verboekend, G. Vilé and J. Pérez-Ramírez, Advanced Functional Materials 2012, 22, 916-928; b) K. Li, J. Valla and J. Garcia-Martinez, ChemCatChem 2014, 6, 46-66.

[34] D. Zhao, Y. Wan and W. Zhou, Ordered Mesoporous Materials, Wiley, 2012, p.

[35] J. H. Clark and D. J. Macquarrie, Organic Process Research \& Development 1997, $1,149-162$.

[36] A. Corma, Chemical Reviews 1995, 95, 559-614.

[37] R. S. Varma, Tetrahedron 2002, 58, 1235-1255.

[38] M. Ogawa, K. Saito and M. Sohmiya, Dalton Transactions 2014, 43, 1034010354

[39] J. H. Clark and D. J. Macquarrie, Chemical Society Reviews 1996, 25, 303-310.

[40] A. Cornelis and P. Laszlo, Synthesis 1985, 1985, 909-918. 
[41] J. H. Clark, A. P. Kybett, D. J. Macquarrie, S. J. Barlow and P. Landon, Journal of the Chemical Society, Chemical Communications 1989, 1353-1354.

[42] G. Nagendrappa, Applied Clay Science 2011, 53, 106-138.

[43] J. H. Clark and D. J. Macquarrie, Chemical Communications 1998, 853-860.

[44] a) G. Sartori and R. Maggi, Chemical Reviews 2006, 106, 1077-1104; b) J. M. Fraile, J. I. García, J. Massam, J. A. Mayoral and E. Pires, Journal of Molecular Catalysis A: Chemical 1997, 123, 43-47.

[45] V. V. Namboodiri, V. Polshettiwar and R. S. Varma, Tetrahedron Letters 2007, $48,8839-8842$.

[46] T. Okuhara, Chemical Reviews 2002, 102, 3641-3666.

[47] S.-O. Kim and J. S. Kim, Macromolecular Research 2002, 10, 174-177.

[48] K. Wilson and H. Clark James in Solid acids and their use as environmentally friendly catalysts in organic synthesis, Vol. 72 2000, p. 1313.

[49] K. Arata, Green Chemistry 2009, 11, 1719-1728.

[50] G. D. Yadav and J. J. Nair, Microporous and Mesoporous Materials 1999, 33, 148.

[51] M. Hino, M. Kurashige, H. Matsuhashi and K. Arata, Thermochimica Acta 2006, $441,35-41$.

[52] B. M. Reddy and M. K. Patil, Chemical Reviews 2009, 109, 2185-2208.

[53] a) S. Kuba, P. Lukinskas, R. K. Grasselli, B. C. Gates and H. Knözinger, Journal of Catalysis 2003, 216, 353-361; b) Z. Helwani, M. R. Othman, N. Aziz, J. Kim and W. J. N. Fernando, Applied Catalysis A: General 2009, 363, 1-10.

[54] W. Zhou, E. I. Ross-Medgaarden, W. V. Knowles, M. S. Wong, I. E. Wachs and C. J. Kiely, Nat Chem 2009, 1, 722-728. 
[55] A. Chaumont and G. Wipff, Physical Chemistry Chemical Physics 2008, 10, 69406953.

[56] I. V. Kozhevnikov, Applied Catalysis A: General 2003, 256, 3-18.

[57] B. M. Devassy and S. B. Halligudi, Journal of Catalysis 2005, 236, 313-323.

[58] T. Blasco, A. Corma, A. Martínez and P. Martínez-Escolano, Journal of Catalysis 1998, 177, 306-313.

[59] A. Corma and H. García, Chemical Reviews 2003, 103, 4307-4366.

[60] a) E. J. M. Hensen, D. G. Poduval, P. C. M. M. Magusin, A. E. Coumans and J. A. R. v. Veen, Journal of Catalysis 2010, 269, 201-218; b) G. Crépeau, V. Montouillout, A. Vimont, L. Mariey, T. Cseri and F. Maugé, The Journal of Physical Chemistry B 2006, 110, 15172-15185.

[61] A. Omegna, J. A. van Bokhoven and R. Prins, The Journal of Physical Chemistry B 2003, 107, 8854-8860.

[62] a) A. Corma, A. Martínez and V. Martínez-Soria, Journal of Catalysis 2001, 200, 259-269; b) C. R. Marcilly, Topics in Catalysis 2000, 13, 357-366.

[63] A. Corma, M. S. Grande, V. Gonzalez-Alfaro and A. V. Orchilles, Journal of Catalysis 1996, 159, 375-382.

[64] W. F. Hoelderich, Catalysis Today 2000, 62, 115-130.

[65] J. L. Provis, G. C. Lukey and J. S. J. van Deventer, Chemistry of Materials 2005, 17, 3075-3085.

[66] B. E. Glad and W. M. Kriven, Journal of the American Ceramic Society 2013, 96, 3643-3649.

[67] A. T. Durant, K. J. D. MacKenzie and H. Maekawa, Dalton Transactions 2011, $40,4865-4870$.

[68] J. Davidovits, Journal of thermal analysis 1989, 35, 429-441. 
[69] V. F. F. Barbosa, K. J. D. MacKenzie and C. Thaumaturgo, International Journal of Inorganic Materials 2000, 2, 309-317.

[70] J. Davidovits, Geopolymer Chemistry and Applications, Geopolymer Institute, 2008, p.

[71] a) P. Duxson, A. Fernández-Jiménez, J. L. Provis, G. C. Lukey, A. Palomo and J. S. J. van Deventer, Journal of Materials Science 2007, 42, 2917-2933; b) X. Yao, Z. Zhang, H. Zhu and Y. Chen, Thermochimica Acta 2009, 493, 49-54.

[72] R. A. Fletcher, K. J. D. MacKenzie, C. L. Nicholson and S. Shimada, Journal of the European Ceramic Society 2005, 25, 1471-1477.

[73] B. Singh, G. Ishwarya, M. Gupta and S. K. Bhattacharyya, Construction and Building Materials 2015, 85, 78-90.

[74] a) M. Alzeer and K. J. D. MacKenzie, Journal of Materials Science 2012, 47, 6958-6965; b) M. Alzeer and K. MacKenzie, Applied Clay Science 2013, 75-76, 148152.

[75] J. G. S. van Jaarsveld and J. S. J. van Deventer, Cement and Concrete Research 1999, 29, 1189-1200.

[76] K. J. D. MacKenzie in 28 - Innovative applications of inorganic polymers (geopolymers), Woodhead Publishing, Oxford, 2015, pp. 777-805.

[77] M. Alzeer, R. A. Keyzers and K. J. D. MacKenzie, Ceramics International 2014, 40, 3553-3560.

[78] P. Sazama, O. Bortnovsky, J. Dědeček, Z. Tvarůžková and Z. Sobalík, Catalysis Today 2011, 164, 92-99.

[79] a) J. R. Gasca-Tirado, A. Manzano-Ramírez, P. A. Vazquez-Landaverde, E. I. Herrera-Díaz, M. E. Rodríguez-Ugarte, J. C. Rubio-Ávalos, V. Amigó-Borrás and M. Chávez-Páez, Materials Letters 2014, 134, 222-224; b) J. R. Gasca-Tirado, A. Manzano-Ramírez, C. Villaseñor-Mora, M. S. Muñiz-Villarreal, A. A. ZaldivarCadena, J. C. Rubio-Ávalos, V. A. Borrás and R. N. Mendoza, Microporous and Mesoporous Materials 2012, 153, 282-287. 
[80] M. Fallah, K. J. D. MacKenzie, J. V. Hanna and S. J. Page, Journal of Materials Science 2015, 50, 7374-7383.

[81] M. Falah, K. J. D. MacKenzie, R. Knibbe, S. J. Page and J. V. Hanna, Journal of Hazardous Materials 2016, 318, 772-782.

[82] S. Sharma, D. Medpelli, S. Chen and D.-K. Seo, RSC Advances 2015, 5, 6545465461 .

[83] S. J. O'Connor, K. J. D. MacKenzie, M. E. Smith and J. V. Hanna, Journal of Materials Chemistry 2010, 20, 10234-10240.

[84] R. Baran, Y. Millot, T. Onfroy, J.-M. Krafft and S. Dzwigaj, Microporous and Mesoporous Materials 2012, 163, 122-130.

[85] J. L. Agudelo, B. Mezari, E. J. M. Hensen, S. A. Giraldo and L. J. Hoyos, Applied Catalysis A: General 2014, 488, 219-230.

[86] D. Verboekend and J. Perez-Ramirez, Catalysis Science \& Technology 2011, 1, 879-890.

[87] G. T. Kerr, The Journal of Physical Chemistry 1968, 72, 2594-2596.

[88] G. Perego, Catalysis Today 1998, 41, 251-259.

[89] G. Busca, Heterogeneous Catalytic Materials: Solid State Chemistry, Surface Chemistry and Catalytic Behaviour, Elsevier Science, 2014, p. 23-35.

[90] a) L. Peng, Y. Liu, N. Kim, J. E. Readman and C. P. Grey, Nat Mater 2005, 4, 216219; b) A. L. Blumenfeld and J. J. Fripiat, Magnetic Resonance in Chemistry 1999, 37, S118-S125.

[91] J. D. M. Kenneth and E. S. Mark in Multinuclear Solid-State Nuclear Magnetic Resonance of Inorganic Materials, Vol. 6 Pergamon, 2002.

[92] J. V. Hanna and M. E. Smith, Solid State Nuclear Magnetic Resonance 2010, 38, $1-18$. 
[93] R. F. Egerton, Physical Principles of Electron Microscopy: An Introduction to TEM, SEM, and AEM, Springer US, 2006, p. 1-151.

[94] P. J. Goodhew, J. Humphreys and R. Beanland, Electron Microscopy and Analysis, Third Edition, Taylor \& Francis, 2000, p. 66-167.

[95] A. E. Taylor in Microwave synthesis and occlusion reactions of zeolites, Vol. $\mathrm{PhD}$ Thesis, The University of Birmingham, 2007.

[96] W. E. Farneth and R. J. Gorte, Chemical Reviews 1995, 95, 615-635.

[97] H. Huo, L. Peng, Z. Gan and C. P. Grey, Journal of the American Chemical Society 2012, 134, 9708-9720.

[98] G. Busca, Heterogeneous Catalytic Materials: Solid State Chemistry, Surface Chemistry and Catalytic Behaviour, Elsevier Science, 2014, p. 57-101.

[99] a) G. P. Heitmann, G. Dahlhoff and W. F. Hölderich, Journal of Catalysis 1999, 186, 12-19; b) J. Kim, W. Park and R. Ryoo, ACS Catalysis 2011, 1, 337-341.

[100] a) O. M. Busch, W. Brijoux, S. Thomson and F. Schüth, Journal of Catalysis 2004, 222, 174-179; b) M. Guisnet, P. Ayrault, C. Coutanceau, M. Fernanda Alvarez and J. Datka, Journal of the Chemical Society, Faraday Transactions 1997, 93, 16611665.

[101] C. A. Emeis, Journal of Catalysis 1993, 141, 347-354.

[102] S. C. Loureiro Dias, J. L. de Macedo and J. A. Dias, Physical Chemistry Chemical Physics 2003, 5, 5574-5579.

[103] K. S. Sing, Pure and applied chemistry 1985, 57, 603-619.

[104] G. Limousin, J. P. Gaudet, L. Charlet, S. Szenknect, V. Barthès and M. Krimissa, Applied Geochemistry 2007, 22, 249-275.

[105] M. A. Vannice, Kinetics of Catalytic Reactions, Springer US, 2005, p.

[106] A. Galarneau, F. Villemot, J. Rodriguez, F. Fajula and B. Coasne, Langmuir 2014, 30, 13266-13274. 
[107] O. Deutschmann, H. Knözinger, K. Kochloefl and T. Turek in Heterogeneous Catalysis and Solid Catalysts, Wiley-VCH Verlag GmbH \& Co. KGaA, 2000, pp. 5266.

[108] U. Nobbmann, M. Connah, B. Fish, P. Varley, C. Gee, S. Mulot, J. Chen, L. Zhou, Y. Lu, F. Sheng, J. Yi and S. E. Harding, Biotechnology and Genetic Engineering Reviews 2007, 24, 117-128.

[109] T. Bligaard, R. M. Bullock, C. T. Campbell, J. G. Chen, B. C. Gates, R. J. Gorte, C. W. Jones, W. D. Jones, J. R. Kitchin and S. L. Scott, ACS Catalysis 2016, 6, 25902602.

[110] J. Haber, Pure and applied chemistry 1991, 63, 1227-1246.

[111] K. V. Kumar, K. Porkodi and F. Rocha, Catalysis Communications 2008, 9, 8284.

[112] M. Boudart, Chemical Reviews 1995, 95, 661-666.

[113] a) F. Shi, M. K. Tse, M.-M. Pohl, A. Brückner, S. Zhang and M. Beller, Angewandte Chemie International Edition 2007, 46, 8866-8868; b) J. A. Lopez-Ruiz and R. J. Davis, Green Chemistry 2014, 16, 683-694.

[114] S. Mukherjee and M. A. Vannice, Journal of Catalysis 2006, 243, 108-130.

[115] a) F. Notheisz, A. g. Zsigmond, M. 1. Barto'k, Z. Szegletes and G. V. Smith, Applied Catalysis A: General 1994, 120, 105-114; b) G. Guo, K. Lian, F. Gu, D. Han and Z. Wang, Chemical Communications 2014, 50, 13575-13577.

[116] R. J. Madon and M. Boudart, Industrial \& Engineering Chemistry Fundamentals 1982, 21, 438-447.

[117] J. Zhu, M. Li, M. Lu and J. Zhu, Catalysis Science \& Technology 2013, 3, 737744.

[118] S. N. Koyande, R. G. Jaiswal and R. V. Jayaram, Industrial \& Engineering Chemistry Research 1998, 37, 908-913. 
[119] F. J. V. Santos, C. A. Nieto de Castro, J. H. Dymond, N. K. Dalaouti, M. J. Assael and A. Nagashima, Journal of Physical and Chemical Reference Data 2006, 35, 1-8.

[120] E. Baum, Chemical Property Estimation: Theory and Application, Taylor \& Francis, 1997, p. 83.

[121] M. Anilkumar and W. F. Hoelderich, Applied Catalysis B: Environmental 2015, 165, 87-93.

[122] J. Zhang, Y. Lu, K. Wang and G. Luo, Industrial \& Engineering Chemistry Research 2013, 52, 6377-6381.

[123] H. Ichihashi and H. Sato, Applied Catalysis A: General 2001, 221, 359-366.

[124] a) G. P. Heitmann, G. Dahlhoff, J. P. M. Niederer and W. F. Hölderich, Journal of Catalysis 2000, 194, 122-129; b) B. Bonelli, L. Forni, A. Aloise, J. B. Nagy, G. Fornasari, E. Garrone, A. Gedeon, G. Giordano and F. Trifirò, Microporous and Mesoporous Materials 2007, 101, 153-160.

[125] a) E. G. Vaschetto, S. G. Casuscelli and G. A. Eimer, Microporous and Mesoporous Materials 2016, 221, 175-181; b) R. Kumar, N. Enjamuri, J. K. Pandey, D. Sen, S. Mazumder, A. Bhaumik and B. Chowdhury, Applied Catalysis A: General 2015, 497, 51-57.

[126] a) E. Gianotti, M. Manzoli, M. E. Potter, V. N. Shetti, D. Sun, J. Paterson, T. M. Mezza, A. Levy and R. Raja, Chemical Science 2014, 5, 1810-1819; b) M. Anilkumar and W. F. Hoelderich, Journal of Catalysis 2012, 293, 76-84.

[127] H. Ichihashi, M. Ishida, A. Shiga, M. Kitamura, T. Suzuki, K. Suenobu and K. Sugita, Catalysis Surveys from Asia 2003, 7, 261-270.

[128] a) N. R. Shiju, M. AnilKumar, W. F. Hoelderich and D. R. Brown, The Journal of Physical Chemistry C 2009, 113, 7735-7742; b) N. R. Shiju, H. M. Williams and D. R. Brown, Applied Catalysis B: Environmental 2009, 90, 451-457; c) M. Opanasenko, M. Shamzhy, M. Lamač and J. Čejka, Catalysis Today 2013, 204, 94-100.

[129] a) Y.-M. Chung and H.-K. Rhee, Journal of Molecular Catalysis A: Chemical 2001, 175, 249-257; b) M. A. Camblor, A. Corma, H. García, V. Semmer-Herlédan and 
S. Valencia, Journal of Catalysis 1998, 177, 267-272; c) C. Ngamcharussrivichai, P. Wu and T. Tatsumi, Applied Catalysis A: General 2005, 288, 158-168.

[130] a) C. Ngamcharussrivichai, P. Wu and T. Tatsumi, Journal of Catalysis 2004, 227, 448-458; b) X. Wang, C.-C. Chen, S.-Y. Chen, Y. Mou and S. Cheng, Applied Catalysis A: General 2005, 281, 47-54.

[131] a) Z. Li, R. Ding, Z. Lu, S. Xiao and X. Ma, Journal of Molecular Catalysis A: Chemical 2006, 250, 100-103; b) N. C. Marziano, L. Ronchin, C. Tortato, A. Vavasori and C. Badetti, Journal of Molecular Catalysis A: Chemical 2007, 277, 221-232; c) N. C. Marziano, L. Ronchin, C. Tortato, A. Vavasori and M. Bortoluzzi, Journal of Molecular Catalysis A: Chemical 2008, 290, 79-87.

[132] a) A. Maia, D. C. M. Albanese and D. Landini, Tetrahedron 2012, 68, 1947-1950; b) X. Liu, L. Xiao, H. Wu, Z. Li, J. Chen and C. Xia, Catalysis Communications 2009, 10, 424-427; c) R. Kore and R. Srivastava, Journal of Molecular Catalysis A: Chemical 2013, 376, 90-97.

[133] a) A.-B. Fernández, A. Marinas, T. Blasco, V. Fornés and A. Corma, Journal of Catalysis 2006, 243, 270-277; b) A. B. Fernández, I. Lezcano-Gonzalez, M. Boronat, T. Blasco and A. Corma, Journal of Catalysis 2007, 249, 116-119.

[134] V. R. R. Marthala, Y. Jiang, J. Huang, W. Wang, R. Gläser and M. Hunger, Journal of the American Chemical Society 2006, 128, 14812-14813.

[135] M. T. Nguyen, G. Raspoet and L. G. Vanquickenborne, Journal of the American Chemical Society 1997, 119, 2552-2562.

[136] A. B. Fernandez, I. Lezcano-Gonzalez, M. Boronat, T. Blasco and A. Corma, Physical Chemistry Chemical Physics 2009, 11, 5134-5141.

[137] W. K. W. Lee and J. S. J. van Deventer, Langmuir 2003, 19, 8726-8734.

[138] A. Zukal, C. O. Arean, M. R. Delgado, P. Nachtigall, A. Pulido, J. Mayerová and J. Čejka, Microporous and Mesoporous Materials 2011, 146, 97-105.

[139] Y. Chen, Z. Guo, T. Chen and Y. Yang, Journal of Catalysis 2010, 275, 11-24. 
[140] Y.-J. Ji, H. Xu, D.-R. Wang, L. Xu, P. Ji, H. Wu and P. Wu, ACS Catalysis 2013, 3, 1892-1901.

[141] V. Gutmann, Electrochimica Acta 1976, 21, 661-670.

[142] D. Mao, Z. Long, Y. Zhou, J. Li, X. Wang and J. Wang, RSC Advances 2014, 4, 15635-15641.

[143] M. Rueping and B. J. Nachtsheim, Beilstein Journal of Organic Chemistry 2010, 6,6 .

[144] in Zion Market Research, 2016, Vol. 2016

[145] M. Osman, S. Al-Khattaf, U. Diaz, C. Martinez and A. Corma, Catalysis Science \& Technology 2016, 6, 3166-3181.

[146] D. Yin, C. Li, L. Tao, N. Yu, S. Hu and D. Yin, Journal of Molecular Catalysis A: Chemical 2006, 245, 260-265.

[147] a) K. R. Devi, P. B. Sreeja and S. Sugunan, International Nano Letters 2013, 3, 40; b) A. P. Singh and A. K. Pandey, Catalysis Letters 1999, 60, 157-159.

[148] a) X. Li, R. Prins and J. A. van Bokhoven, Journal of Catalysis 2009, 262, 257265; b) H. Jin, M. B. Ansari, E.-Y. Jeong and S.-E. Park, Journal of Catalysis 2012, 291, 55-62.

[149] a) J. Li, C. Xu, L.-L. Lou, C. Zhang, K. Yu, B. Qi, S. Liu and Y. Wang, Catalysis Communications 2013, 38, 59-62; b) J. J. Chiu, D. J. Pine, S. T. Bishop and B. F. Chmelka, Journal of Catalysis 2004, 221, 400-412; c) S. Jun and R. Ryoo, Journal of Catalysis 2000, 195, 237-243; d) H. Hashimoto, A. Itadani, T. Kudoh, Y. Kuroda, M. Seno, Y. Kusano, Y. Ikeda, M. Nakanishi, T. Fujii and J. Takada, ACS Applied Materials \& Interfaces 2013, 5, 518-523.

[150] a) M. H. Al-Hazmi and A. W. Apblett, Catalysis Science \& Technology 2011, 1, 621-630; b) F. Adam and J. Andas, Journal of Colloid and Interface Science 2007, 311, 135-143; c) H. Hentit, K. Bachari, M. S. Ouali, M. Womes, B. Benaichouba and J. C. Jumas, Journal of Molecular Catalysis A: Chemical 2007, 275, 158-166; d) J. He, J. 
Chen, L. Ren, Y. Wang, C. Teng, M. Hong, J. Zhao and B. Jiang, ACS Applied Materials \& Interfaces 2014, 6, 2718-2725.

[151] V. D. Sarca and K. K. Laali, Green Chemistry 2006, 8, 615-620.

[152] a) S. Svelle, U. Olsbye, K.-P. Lillerud, S. Kolboe and M. Bjørgen, Journal of the American Chemical Society 2006, 128, 5618-5619; b) J. Čejka and B. Wichterlová, Catalysis Reviews 2002, 44, 375-421.

[153] K. J. D. MacKenzie and M. E. Smith, Multinuclear Solid-State Nuclear Magnetic Resonance of Inorganic Materials, Elsevier Science, 2002, p. 201-324.

[154] A. Vinu, D. P. Sawant, K. Ariga, M. Hartmann and S. B. Halligudi, Microporous and Mesoporous Materials 2005, 80, 195-203.

[155] C. L. Yaws, Thermophysical Properties of Chemicals and Hydrocarbons, Elsevier Science, 2014, p. 697.

[156] D. Khale and R. Chaudhary, Journal of Materials Science 2007, 42, 729-746.

[157] a) C. Khatri, M. K. Mishra and A. Rani, Fuel Processing Technology 2010, 91, 1288-1295; b) Y. Wang, H. Song and X. Sun, RSC Advances 2016, 6, 107239-107245.

[158] Z. T. Yao, X. S. Ji, P. K. Sarker, J. H. Tang, L. Q. Ge, M. S. Xia and Y. Q. Xi, Earth-Science Reviews 2015, 141, 105-121.

[159] Heidrich C.; Feuerborn H.; Weir A. in Coal Combustion Products: a Global Perspective, Vol. Lexington, Kentucky, USA, 2013.

[160] S. Wang and H. Wu, Journal of Hazardous Materials 2006, 136, 482-501.

[161] S. Wang, Environmental Science \& Technology 2008, 42, 7055-7063.

[162] B. W. Ramme, M. P. Tharaniyil and W. E. P. Company, Wisconsin Electric Energies Coal Combustion Products Utilization Handbook, We Energies, 2004, p. 9 34.

[163] V. C. Pandey and N. Singh, Agriculture, Ecosystems \& Environment 2010, 136, $16-27$. 
[164] A. González, R. Navia and N. Moreno, Waste Management \& Research 2009, 27, 976-987.

[165] J. Temuujin, A. van Riessen and K. J. D. MacKenzie, Construction and Building Materials 2010, 24, 1906-1910.

[166] a) S. Mohan and R. Gandhimathi, Journal of Hazardous Materials 2009, 169, 351-359; b) G. Itskos, N. Koukouzas, C. Vasilatos, I. Megremi and A. Moutsatsou, Journal of Hazardous Materials 2010, 183, 787-792.

[167] F. A. Torralvo and C. Fernández-Pereira, Minerals Engineering 2011, 24, 35-41.

[168] a) R. S. Blissett and N. A. Rowson, Fuel 2012, 97, 1-23; b) C. T. Kniess, J. C. de Lima, P. B. Prates, N. C. Kuhnen and H. G. Riella, Journal of Non-Crystalline Solids 2007, 353, 4819-4822.

[169] S. Wang and G. Q. Lu in Effect of chemical treatment on ni/fly-ash catalysts in methane reforming with carbon dioxide, Vol. Volume 167 Eds.: M. S. Fábio Bellot Noronha and S.-A. Eduardo Falabella), Elsevier, 2007, pp. 275-280.

[170] X. Xuan, C. Yue, S. Li and Q. Yao, Fuel 2003, 82, 575-579.

[171] M. S. Kotwal, P. S. Niphadkar, S. S. Deshpande, V. V. Bokade and P. N. Joshi, Fuel 2009, 88, 1773-1778.

[172] Z. Shi, S. Yao and C. Sui, Catalysis Science \& Technology 2011, 1, 817-822.

[173] S. Muhammad, E. Saputra, H. Sun, J. d. C. Izidoro, D. A. Fungaro, H. M. Ang, M. O. Tade and S. Wang, RSC Advances 2012, 2, 5645-5650.

[174] O. Babajide, N. Musyoka, L. Petrik and F. Ameer, Catalysis Today 2012, 190, 54-60.

[175] M. C. Manique, L. V. Lacerda, A. K. Alves and C. P. Bergmann, Fuel 2017, 190, 268-273.

[176] K. Ojha, N. C. Pradhan and A. N. Samanta, Journal of Chemical Technology \& Biotechnology 2006, 81, 659-666. 
[177] A. O. Dhokte, S. L. Khillare, M. K. Lande and B. R. Arbad, Journal of Industrial and Engineering Chemistry 2011, 17, 742-746.

[178] a) Y. Zhang and L. Liu, Particuology 2013, 11, 353-358; b) A. Strini, G. Roviello, L. Ricciotti, C. Ferone, F. Messina, L. Schiavi, D. Corsaro and R. Cioffi, Materials 2016, 9, 513.

[179] Y. Zhang, W. Sun and Z. Li, Journal of Wuhan University of Technology-Mater. Sci. Ed. 2008, 23, 522-527.

[180] X. Chen, F. Chen, F. Liu, X. Yan, W. Hu, G. Zhang, L. Tian, Q. Xia and X. Chen, Catalysis Science \& Technology 2016, 6, 4184-4191.

[181] E. Díaz, S. Ordóñez, A. Vega, A. Auroux and J. Coca, Applied Catalysis A: General 2005, 295, 106-115.

[182] R. H. Vekariya and J. Aubé, Organic Letters 2016, 18, 3534-3537.

[183] M. Mu, L. Chen, Y. Liu, W. Fang and Y. Li, RSC Advances 2014, 4, 3695136958.

[184] M. Ammar, S. Jiang and S. Ji, Journal of Solid State Chemistry 2016, 233, 303310.

[185] S. Jiang, J. Yan, F. Habimana and S. Ji, Catalysis Today 2016, 264, 83-90.

[186] G. K. S. Prakash, F. Paknia, A. Kulkarni, A. Narayanan, F. Wang, G. Rasul, T. Mathew and G. A. Olah, Journal of Fluorine Chemistry 2015, 171, 102-112.

[187] R. Parella, Naveen, A. Kumar and S. A. Babu, Tetrahedron Letters 2013, 54, 1738-1742.

[188] A. Perrier, M. Keller, A.-M. Caminade, J.-P. Majoral and A. Ouali, Green Chemistry 2013, 15, 2075-2080. 